Florida International University FIU Digital Commons

FIU Electronic Theses and Dissertations

University Graduate School

$11-9-2016$

\title{
Wind Performance Based Design for High-Rise Buildings
}

Alireza Mohammadi

amoha058@fiu.edu

DOI: 10.25148 /etd.FIDC001201

Follow this and additional works at: https://digitalcommons.fiu.edu/etd

Part of the Structural Engineering Commons

\section{Recommended Citation}

Mohammadi, Alireza, "Wind Performance Based Design for High-Rise Buildings" (2016). FIU Electronic Theses and Dissertations. 3032.

https://digitalcommons.fiu.edu/etd/3032

This work is brought to you for free and open access by the University Graduate School at FIU Digital Commons. It has been accepted for inclusion in FIU Electronic Theses and Dissertations by an authorized administrator of FIU Digital Commons. For more information, please contact dcc@fiu.edu. 


\title{
FLORIDA INTERNATIONAL UNIVERSITY
}

\author{
Miami, Florida
}

WIND PERFORMANCE BASED DESIGN FOR HIGH-RISE BUILDINGS

A dissertation submitted in partial fulfillment of the

requirements for the degree of

DOCTOR OF PHILOSOPHY

in

CIVIL ENGINEERING

by

Alireza Mohammadi

2016 
To: Interim Dean Ranu Jung

College of Engineering and Computing

This dissertation, written by Alireza Mohammadi, and entitled Wind Performance Based Design for High-Rise Buildings, having been approved in respect to style and intellectual content, is referred to you for judgment.

We have read this dissertation and recommend that it be approved.

Ali Mostafavi

Ioannis Zisis

Arindam Gan Chowdhury

Emil Simiu

Larry Griffis

Peter Irwin

Atorod Azizinamini, Major Professor

Date of Defense: November 9, 2016

The dissertation of Alireza Mohammadi is approved.

Interim Dean Ranu Jung

College of Engineering and Computing

Andrés G. Gil

Vice President for Research and Economic Development and Dean of the University Graduate School

Florida International University, 2016 
(C) Copyright 2016 by Alireza Mohammadi All rights reserved. 


\section{DEDICATION}

I dedicate this dissertation to my beloved fiancé (Basira), my loving parents, and my caring sister and brother. Without their patience, understanding, support, and most of all love, the completion of this work would not have been possible. 


\section{ACKNOWLEDGMENTS}

First and foremost, I have to thank my dear parents for their love and support throughout my life. Thank you both for giving me strength and enthusiasm to stand even when circumstances seem impossible. To my fiancé, Basira, thank you for your assistance, your encouragement and your patience. My dear sister and brother deserve my wholehearted thanks as well.

I would like to express my sincere appreciation and gratitude to my advisor Dr. Atorod Aziznamini for his guidance and support, not only during this dissertation project, but also during the more than four years of my Ph.D. program. His support and inspiring suggestions have been precious for me.

I am also very grateful to Dr. Peter Irwin and Mr. Larry Griffis, who have been a constant source of guidance and encouragement during this research project. In addition, Dr. Emil Simiu, Dr. Arindam Chowdhury, Dr. Ionnis Zisis and Dr. Ali Mostafavi deserve special thanks, as my dissertation committee members and advisors. Special thanks to Dr. Finely Charney and Dr. Johnn Judd for their thoughtful and helpful comments which greatly contributed to this research effort.

I would also like to thank Dr. Saeed E. Doust, Dr. Aaron Yakel and Dr. Jawad Gull from whom I gained knowledge and research skills. I would also like to thank my fellow graduate students, Ramin Taghinezad, Huy Pham, Mohammadreza Shafieifar, Mahsa Farzad, Alireza Valikhani and Azadeh Jaberi for their friendship and collaboration.

A special thank goes to Edgar Polo, Walter Conklin and Roy Liu-Marques for helping me on the experimental works. I would like to thank Dr. Hector Fuentes, Haydee Caneda, Rachel Garcia, Carlton Ng, Jessica Teran, and administrative staff at Florida International University for helping out with the department and university policies and procedures.

Finally, I extend my thanks to all those unnamed individuals whose encouragement and corporation directly or indirectly contributed to the completion of this dissertation. 


\section{ABSTRACT OF THE DISSERTATION \\ WIND PERFORMANCE-BASED DESIGN FOR HIGH-RISE BUILDINGS}

by

\section{Alireza Mohammadi}

Florida International University, 2016

Miami, Florida

\section{Professor Atorod Azizinamini, Major Professor}

The rapid growth of high-rise high-density urban areas in coastal and near coastal, hurricaneprone cities has been observed globally and in the United States in recent decades. Favored by modern urban growth and planning policies, this trend is expected to accelerate in future. Recent climate change studies suggest a significant increase in the destructiveness of hurricanes in past 30 years by both increases in lifetime and intensity of hurricanes. Current prescriptive wind design approach does not provide transparent methods and criteria to reliably quantify the performance of buildings as well as the functional requirements necessary to accommodate large populations during extreme wind. Since this approach primarily intends to keep the structural system essentially elastic, the more efficient design may be achievable by allowing controlled inelasticity in structural components. All these facts put a great emphasis on using a reliable wind design and assessment approach evidently describing the performance of high-rise building to wind loads beyond the current design wind loads.

This dissertation presents the development of a wind performance-based engineering approach and its practical implementation for three, 47-, 40- and 30-story steel moment frame high-rise buildings. In this study, the nonlinear dynamic responses of the buildings to different wind hazard levels were evaluated by developing 3D nonlinear finite element models and utilizing a wind 
incremental dynamic analysis (IDA) approach. The wind loading for the 47-story building was measured by conducting wind pressure testing on a scaled rigid model at the Wall of Wind (WOW) facility at Florida International University. For two other buildings wind loads were acquired using TPU Aerodynamic Database. Using the IDA results and adopting available wind performance criteria, a wind performance assessment approach was developed representing the estimated performance levels as a function of the basic wind speed. Three types of wind performance were evaluated: structural component performance; cladding performance to windinduced shear deformation; and serviceability motion comfort performance. This evaluation indicated remarkable lateral capacity associated with allowing controlled structural nonlinearity, in contrast to considerations required to assure acceptable serviceability and non-structural (e.g. cladding) performances. 


\section{TABLE OF CONTENTS}

CHAPTER

PAGE

CHAPTER I: INTRODUCTION ………………………………………………….... 1

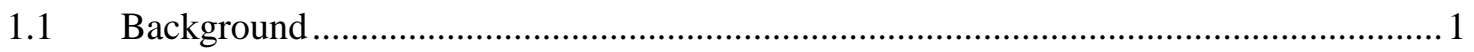

1.2 Performance of High-Rise Buildings in Recent Major Hurricanes................................... 3

1.3 Current Wind Design of High-Rise Buildings .............................................................

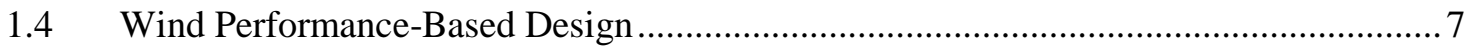

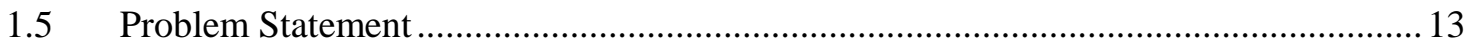

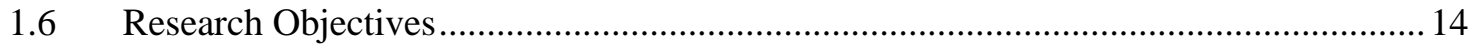

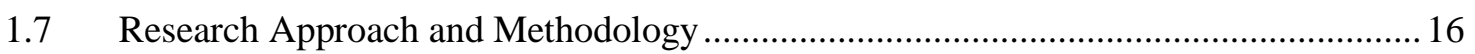

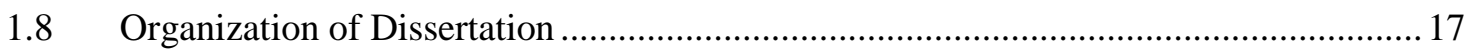

CHAPTER II: DEVELOPMENT OF THE ANALYTICAL PROCEDURE ..............................20

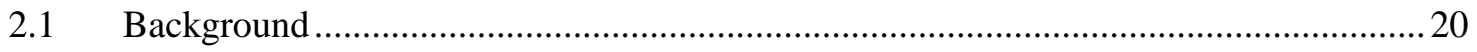

2.2 Description of High-Rise Building Models ................................................................ 22

2.2.1 47-Story High-Rise Building Model .................................................................... 22

2.2.2 30-Story and 40-Story Steel Moment High-Rise Building Models .......................24

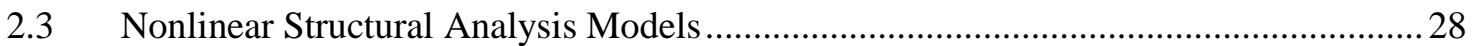

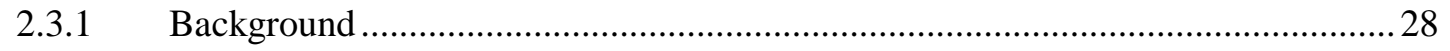

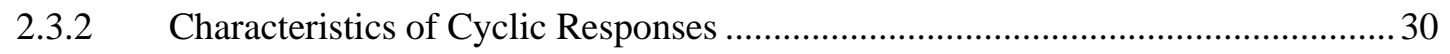

2.3.3 Nonlinear Hysteretic Models of Structural Components ......................................... 35

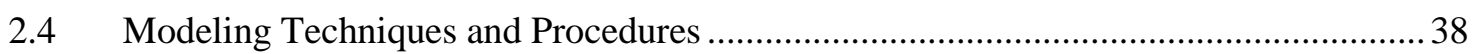

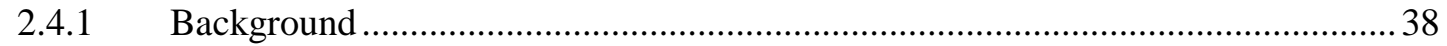

2.4.2 Types of Force Action in Structural Components................................................... 39

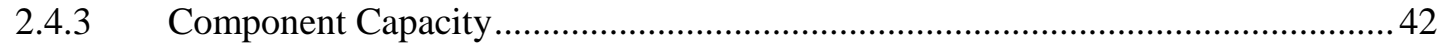

2.4.4 Selecting The Nonlinear Modeling Approach …................................................... 43

2.4.5 Nonlinear Hysteretic Modeling of Steel Beams and Columns ................................. 44

2.4.6 Axial Load- Bending Moment Interaction in Columns ...........................................53

2.4.7 Composite Action Effect On Response of Beams .................................................55

2.4.8 Modeling of Steel Moment-Resisting Connections ................................................55

2.4.9 Modeling The Nonlinearity of Steel Panel Zones................................................. 72

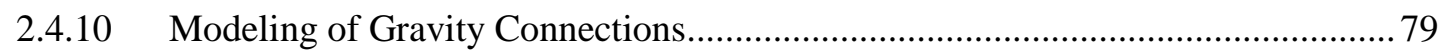

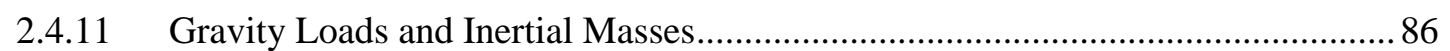

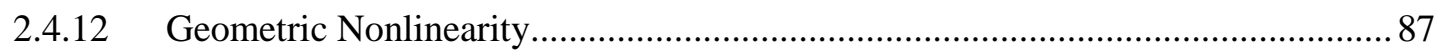

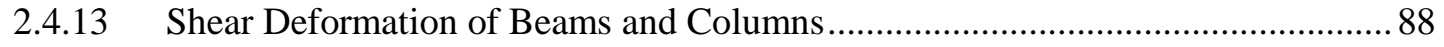




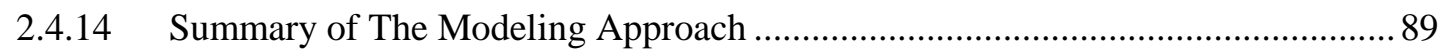

CHAPTER III: VALIDATION OF THE ANALYTICAL APPROACH...................................92

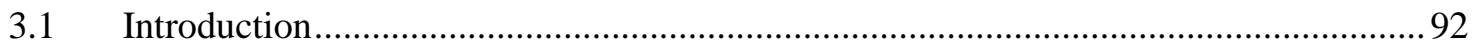

3.2 Validation of Fiber-Section Nonlinear Model …......................................................... 93

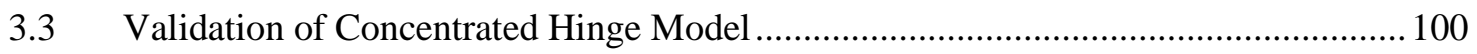

3.4 Validation of The Modeling of Semi-Rigid Connections ........................................... 102

3.5 Validation of Analytical Approach Using 5-Story Shake Table Results ..................... 106

3.6 Validation of Analytical Approach Using 4-Story Shake Table Results ...................... 110

CHAPTER IV: STUDY ON ANALYTICAL MODELING OF DAMPING …....................... 115

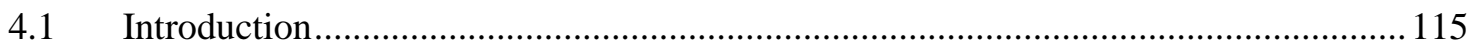

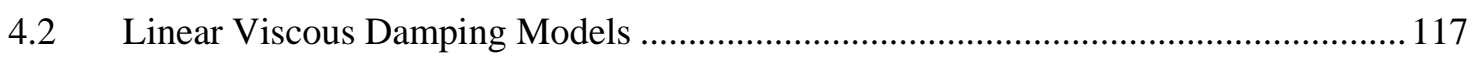

4.3 Inaccurate Dynamic Response of Nonlinear System from Rayleigh Damping Model 119

4.4 Suggestions for Proper Damping Modeling for Inelastic Systems ............................. 120

4.5 Investigation on Damping Modeling in The 47-Story Building Model ....................... 121

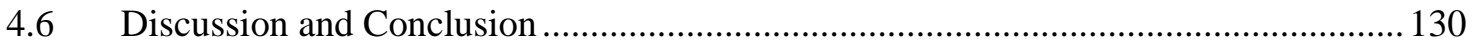

CHAPTER V: WIND PRESSURE TESTING AND WIND DYNAMIC LOADING .............. 132

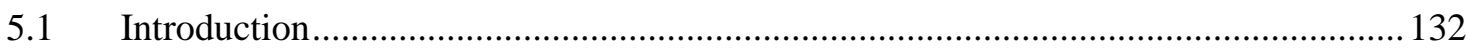

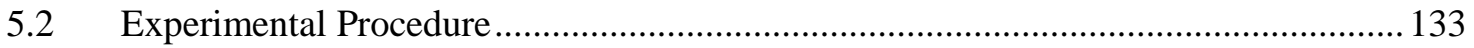

5.2.1 Wind Test Facility and Characteristics of Approaching Flow ............................. 133

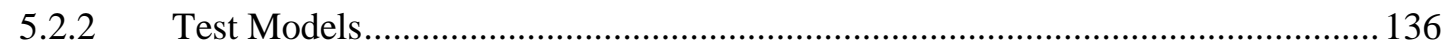

5.2.3 Definition of Studied Parameters ....................................................................... 142

5.2.4 Wind Test Results and Discussion...................................................................... 143

5.2.5 Comparing Results with TPU Wind Tunnel Database ........................................ 147

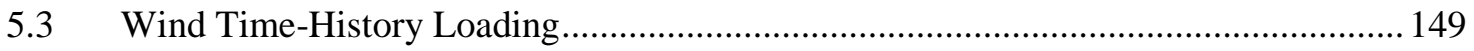

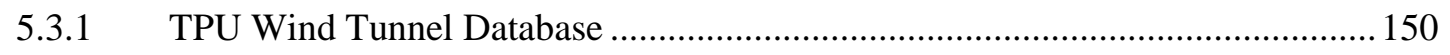

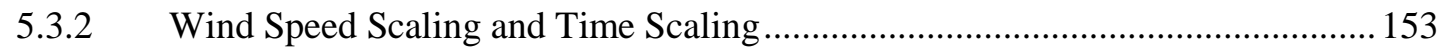

5.3.3 Wind Time-History Point Loads ..................................................................... 155

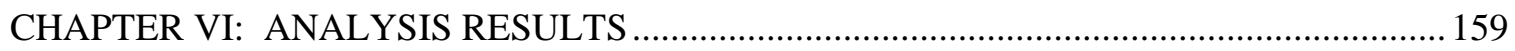

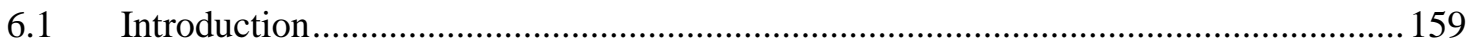

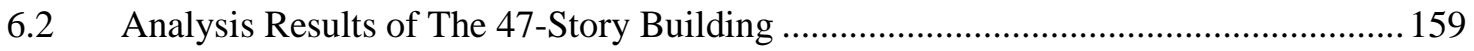

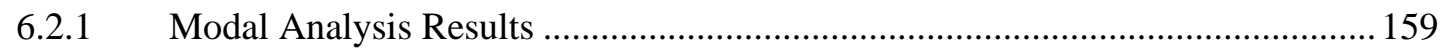

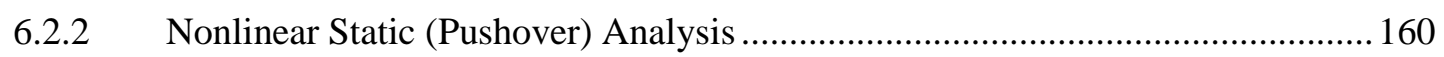

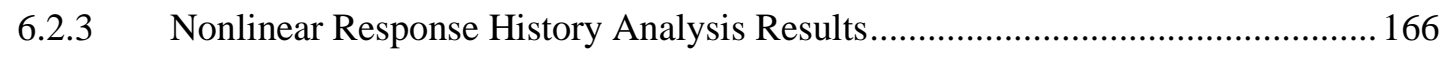

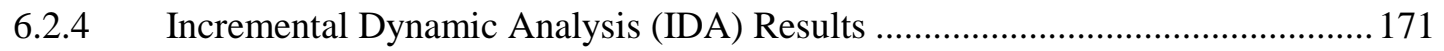




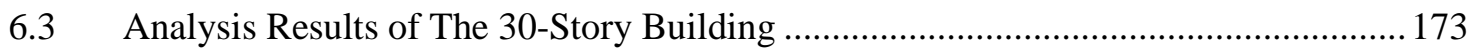

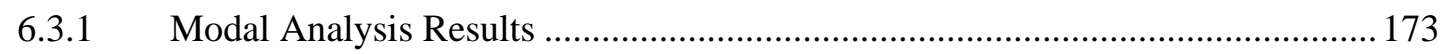

6.3.2 Nonlinear Static (Pushover) Analysis Results ..................................................... 174

6.3.3 Nonlinear Response History Analysis Results................................................... 179

6.3.4 Incremental Dynamic Analysis (Ida) Results .................................................... 181

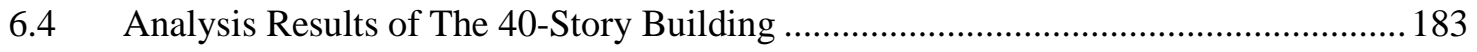

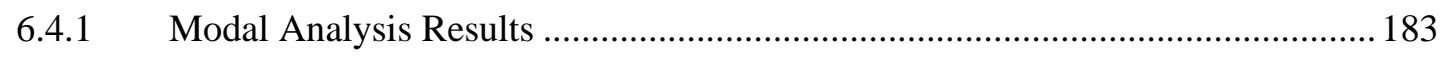

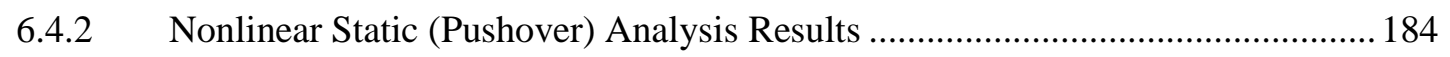

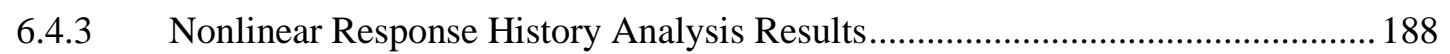

6.4.4 Incremental Dynamic Analysis (Ida) Results ................................................... 189

CHAPTER VII: DEVELOPMENT AND IMPLANTATION OF A WIND PERFORMANCE

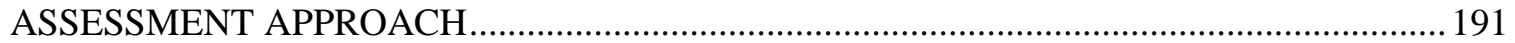

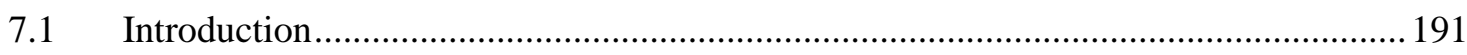

7.2 Development of The Wind Performance Assessment Approach ................................. 192

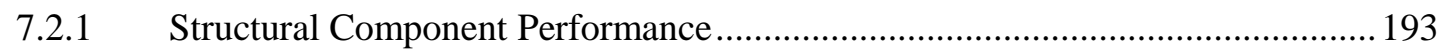

7.2.2 Cladding Performance to Wind-Induced Shear Deformation ................................ 196

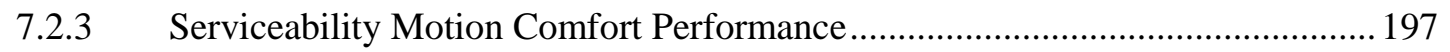

7.3 Wind Performance Assessment of the Case Study Buildings.....................................200

7.3.1 Wind Performance Assessment of The 47-Story Building ................................... 200

7.3.2 Wind Performance Assessment of The 30-Story Building ..................................2 210

7.3.3 Wind Performance Assessment of The 40-Story Building ..................................217

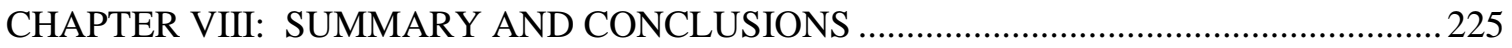

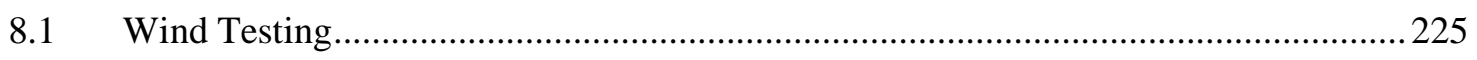

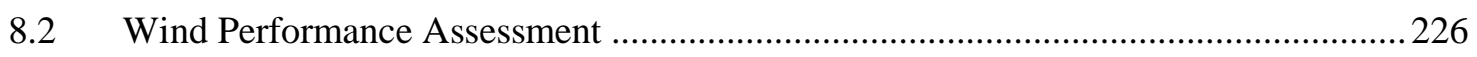

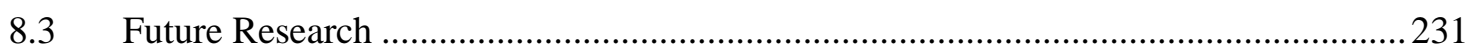

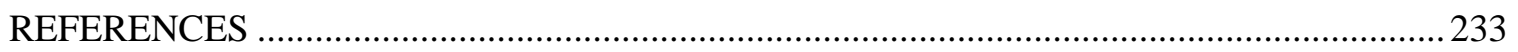

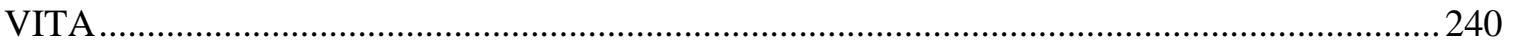




\section{LIST OF TABLES}

TABLE

PAGE

Table 1-1.The Saffir/Simpson hurricane wind scale (NOAA, 2011) ..........................................

Table 2-1. Force actions and their possible response type (ASCE, 2014) ................................. 40

Table 2-2. Determining the reduction factor for the axial load effect on pre-capping and precapping plastic rotations according to ASCE/SEI 41-13 ..............................................54

Table 2-3. Checking the beam web and flange buckling failure mode for the 47-story building

Table 2-4. Summary of modes of failure of the pre-Northridge connections, their controlling parameters and their consideration in the analytical analysis.

Table 2-5. The average parameters for developing calibrated moment-rotation backbone of composite shear tab connections (Elkady \& Lignos, 2015).

Table 2-6. The average parameters for developing pinching and degradation composite shear tab connections cyclic response (Elkady \& Lignos, 2015)

Table 3-1. Compression between story displacement responses obtained in this study and Azizinamini's

Table 3-2. Intensities of the applied excitations and corresponding building's response states and the design stages

Table 4-1. Natural frequencies and the viscous damping coefficients obtained from elastic and inelastic free vibration analyses.......................................................................... 130

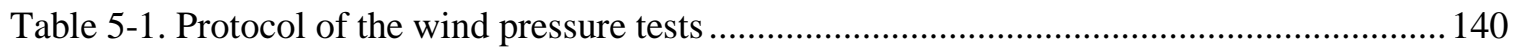

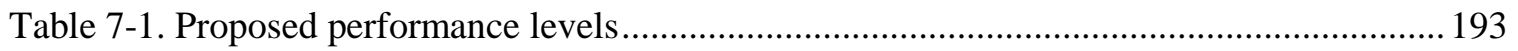

Table 7-2. Serviceability performance criteria related to motion comfort (Chang, 1973)........... 199

Table 7-3. Suggested serviceability criteria related to motion comfort .................................... 199 


\section{LIST OF FIGURES}

FIGURE

PAGE

Figure 1-1.The 30 costliest tropical cyclones to strike the United States, 1900-2010 (NOAA, 2011)

Figure 1-2. Traffic Jam during evacuation from Hurricane Rita, Houston, TX

Figure 1-3. Energy distribution of earthquake and windstorm excitations in frequency

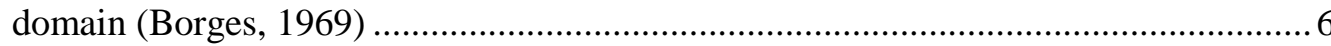

Figure 1-4. PEER's framework for seismic performance-based design (Porter, 2003)................. 9

Figure 1-5. Recommended seismic performance objectives (SEAOC, 2000) .............................. 10

Figure 1-6. Wind Performance objective matrix (Griffis et al., 2012) ....................................... 13

Figure 2-1. The 47-story building; (a) typical floor framing of the underground levels (the above-ground levels shown by the dotted red square); (b) perspective view of the building's shape before the renovation.

Figure 2-2. Floor plan, elevations and beam and column sections of the 30 -story building (Han et al., 2015)

Figure 2-3. Floor plan, elevations and beam and column sections of the 40-story building (Han et al., 2015)

Figure 2-4. Idealized models representing inelasticity for beam-column elements (Deierlein, Reinhorn, \& Willford, 2010)

Figure 2-5. Modes of deterioration (PEER/ATC, 2010) .............................................................. 31

Figure 2-6. Two type of degradations in hysteresis response (FEMA, 2005; FEMA, 2009) .........32

Figure 2-7. Force-displacement backbones; (a) cyclic backbone versus monotonic backbone (Deierlein et al., 2010), (b) cyclic envelope versus capacity boundary (FEMA, 2009)

Figure 2-8. Typical force-deformation backbone for nonlinear dynamic models of components (FEMA, 2009)

Figure 2-9. Basic linearized hysteresis models: (a) bilinear; (b) peak oriented; and (c) pinching (PEER/ATC, 2010)

Figure 2-10. Force-deformation backbones for different types of force actions in structural components

Figure 2-11. ASCE41-13 component response of deformation-controlled actions: (a) forcedeformation relationship, (b) acceptance criteria 
Figure 2-12. Modified IK deterioration model: (a) backbone curve; (b) cyclic response and deterioration characteristics (Lignos \& Krawinkler, 2010a).

Figure 2-13. Failure modes of pre-Northridge connections (FEMA, 2000b). .59

Figure 2-14. Typical beam to column moment-resisting connection in the 47-story building .59

Figure 2-15. Web transverse stiffeners of beams in moment resisting frames of the 47 -story building.

Figure 2-16. Details of flooring, framing and connections in the 47-story building

Figure 2-17. Nonlinear moment-rotation relationship suggested for pre-Northridge connections according to ASCE 41-13

Figure 2-18. Instances of measured moment-rotation behavior of pre-Northridge connections in experimental tests: (a) conducted by Lee et al., (2000); (b) conducted by Engelhardt et al. (1993)

Figure 2-19. Hysteretic moment-rotation relationship of pre-Northridge connections: (a) developed idealized model; (b) moment-rotation relationship of a pre-Northridge connection with $\mathrm{W} 24 \mathrm{x} 84$ beam

Figure 2-20. Panel zone forces and deformation: (a) moment and shear forces due to lateral loads, (b) panel zone distortion.

Figure 2-21.Cyclic shear behavior of a weak panel zone

Figure 2-22. Trilinear shear force - shear distortion response of panel zones (Gupta and Krawinkler, 1999).

Figure 2-23. (a) Simplified analytical model of panel zone (scissors model), (b) panel zone model with rigid boundaries (parallelogram model) (c) two springs model simulating the trilinear response (Gupta and Krawinkler, 1999).

Figure 2-24. Comparison of moment-rotation response of a gravity framing with and without the floor slab (Liu \& Astaneh-Asl, 2000).

Figure 2-25. Typical gravity frame response model: (a) the force-displacement capacity backbone; (b) the hysteretic behavior.

Figure 2-26. (a) Pinching4 material model (b) Idealized moment-rotation backbone curve simulating the response of composite shear tab connections (Elkady \& Lignos, 2015)

Figure 2-27. Moment-rotation backbone and enclosed cyclic response of the 47-story building's gravity connections with $\mathrm{W} 18 \times 40$ beams... 
Figure 2-28. Pushover curve responses of the 47-story building using ASCE 7-10 wind load pattern, with and without gravity load illustrating the P- $\Delta$ geometric nonlinear effect.

Figure 2-29. Pushover curve responses of the 47-story building, using ASCE 7-10 wind load pattern, with and without beams and columns shear deformations. 89

Figure 2-30. Representation of inelasticity modeling in moment-resisting frames

Figure 2-31. Representation of inelasticity modeling in gravity frames

Figure 3-1. Details of the single-bay 2-story model.

Figure 3-2. Typical fiber-section subdividing for the I-shape and box steel members.

Figure 3-3. Giuffré-Menegotto-Pinto constitutive model for steel: (a) typical monotonic behavior (b) typical cyclic behavior with isotropic hardening

Figure 3-4. Pushover analysis results of the three models to compare the accuracy in simulating the nonlinearity.

Figure 3-5. Deformed shape and along-push direction stress contours of the 2-story frame......... 98

Figure 3-6. 2-story steel SMF: (a) the representative building plan ;(b) modeling details .......... 101

Figure 3-7. Comparison between obtained pushover curves of the 2-story frame 102

Figure 3-8. Sample analysis on semi-rigid connections: (a) sample plan frame; (b) details of the top-and-seat and web angle type connections; (c) details of the top-and-seat type connections

Figure 3-9. Nonlinear moment-rotation response of the semi-rigid connections 105

Figure 3-10. Details of the 5-story steel moment frame building (Ryan, Dao, Sato, Sasaki, \& Okazaki, 2012)...

Figure 3-11. Modal shape and natural periods of the 4-story building obtained from both the test and the analytical simulation.

Figure 3-12. Peak story drift ratio of the 5-story building subjected to $35 \%$ Northridge earthquake; the test results versus the analytical simulation results.

Figure 3-13. Pushover results for different methods of panel zone modeling: (a) In $X$ Direction, (b) in Y-direction.

Figure 3-14. Details of the 5-story steel moment frame building 110

Figure 3-15. Modal shape and natural periods of the 4-story building obtained from both the test and the analytical simulation. 
Figure 3-16. 1995 Kobe earthquake ground acceleration recorded at Takatori Station: (a) in $\mathrm{N}-\mathrm{S}$ direction; (b) in E-W direction; and (c) in vertical Direction.

Figure 3-17. Peak story drift ratio of the 4-story building subjected to different intensities of Kobe earthquake; test results versus analytical simulation results: (a) in $\mathrm{X}$ direction; (b) in Y-direction.

Figure 4-1. Rayleigh damping: (a) variation of modal damping ratio with natural frequencies; (b) illustration of MPD and KPD component damping.

Figure 4-2. Elastic free vibration; the roof displacement responses for the four different damping models

Figure 4-3. Frequency domain elastic free vibration response of the 47-story building; using Rayleigh damping based on tangent stiffness matrix

Figure 4-4. Fitted viscous damping to free vibration response of the 47-story building; using Rayleigh damping based on tangent stiffness matrix

Figure 4-5. Nonlinearity in structural members of the 47-story building due to statically pushing by $125 \%$ of ASCE 7-10 wind loading.

Figure 4-6. Inelastic free vibration; the roof displacement responses for the four different damping models

Figure 4-7. Frequency domain inelastic free vibration response of the 47-story building; using Rayleigh damping based on tangent stiffness matrix

Figure 4-8. Fitted viscous damping to inelastic free vibration response of the 47 -story building; using Rayleigh damping based on tangent stiffness matrix

Figure 5-1. 12-fan Wall of Wind; (a) schematic model, (b) intake side, (c) exit side

Figure 5-2. Simulated suburban terrain: (a) ABL profile and (b) turbulence intensity profile .... 135

Figure 5-3. Test Models: (a) Test \#1: isolated prismatic shape model, (b) Test \#2: isolated full building model, (c) Test \#3: full building model and surrounding buildings ..... 137

Figure 5-4. Wind tunnel model details including pressure taps layout on the sides and the roof of the prism-shape model; scaled detail of the penthouse; and scaled detail of the skirt part......

Figure 5-5. Immediate surroundings of the 47-story building, illustration of approaching wind and the notation of the directions....

Figure 5-6. Transfer function of the tubing system

Figure 5-7. Surface mean pressure coefficient distribution for Test \#1 (prismatic shape model) for the wind flow normal to the upstream face. 
Figure 5-8. Surface mean pressure coefficient distribution for Test \#2 (including penthouse and the skirt) for the wind flow normal to the upstream face.

Figure 5-9. Mean base force coefficients versus the wind directions: (a) in the x-direction;

(b) in the y-direction

Figure 5-10. Fluctuating base force coefficients versus the wind directions: (a) in the $\mathrm{x}$ direction; (b) in the y-direction.....

Figure 5-11. Area average $\mathrm{C}_{\mathrm{f}}$ of WOW and TPU results for testing the isolated model: (a) on the windward face; (b) on the left sideward face; (c) on the leeward face; and (d) on the right sideward face.

Figure 5-12. Typical data available on TPU Aerodynamic Database (http://wind.arch.tkougei.ac.jp/system/eng/contents/code/tpu)......

Figure 5-13. Typical pressure channel positioning in TPU Aerodynamic Database and corresponding positions around the building (http://wind.arch.tkougei.ac.jp/system/eng/contents/code/tpu)

Figure 5-14. Illustration of breadth, depth and height of the building (http://wind.arch.tkougei.ac.jp/system/eng/contents/code/tpu)

Figure 5-15. Converting measured loads to the location of the applied time-history point loads

Figure 5-16. Location of applied time-history loads on each building's face for all three buildings

Figure 5-17. Wind time-history loading; (a) typical point load, (b) the added ramp-up portion.158

Figure 6-1. Modal Shapes, natural periods and the types of first five modes of 47-story building model

Figure 6-2 . Wind nonlinear pushover: (a) Pushover curve for X-direction response; (b) Pushover curve in Z-direction response

Figure 6-3. Wind pushover response of the 47-story building; nonlinearity distribution in near-collapse stage in $\mathrm{X}$-direction response

Figure 6-4. Wind pushover response of the 47-story building; nonlinearity distribution in near-collapse stage in Z-direction response.

Figure 6-5. Wind directional dynamic response of the 47-story building: (a) peak story drift ratio; (b) peak base shear

Figure 6-6. Wind dynamic responses for $\mathrm{V}=82 \mathrm{mph}$ wind speed and $180^{\circ}$ wind direction for the three conducted wind pressure tests: (a) peak story drift ratio in X-direction: (b) peak story drift ratio in Z-direction; (c) peak floor acceleration in $\mathrm{X}$ direction: (d) peak floor acceleration in Z-direction. 
Figure 6-7. IDA responses of the 47-story building: (a) peak story drift ratio in X-direction; (b) peak story drift ratio in Z-direction; (c) peak floor acceleration in X-direction;

(d) peak floor acceleration in Z-direction

Figure 6-8. Modal Shapes, natural periods and the type of the first five modes of the 30-story building.....

Figure 6-9 . Wind pushover: (a) pushover curve in X-direction response; (b) Pushover curve in Z-direction response

Figure 6-10. Wind pushover response of the 30-story building; nonlinearity distribution in near-collapse stage in $\mathrm{X}$ direction response.

Figure 6-11. Wind pushover response of the 30-story building; nonlinearity distribution in near-collapse stage in Z-direction response

Figure 6-12. Wind directional dynamic response of the 30-story building: (a) peak story drift response; (b) peak base shear repose

Figure 6-13. Wind directional dynamic response of the 30-story building: (a) peak drift story ratio in X-Direction; (b) peak drift story ratio in Z-Direction; (a) peak floor acceleration in X-Direction; (b) peak floor acceleration in Z-Direction

Figure 6-14. IDA responses of the 30-story building: (a) peak story drift ratio in X-direction;

(b) peak story drift ratio in Z-direction; (c) peak floor acceleration in X-direction;

(d) peak floor acceleration in Z-direction

Figure 6-15. Modal Shapes, natural periods and the type of the first five modes of the 40story building

Figure 6-16 . Wind pushover: (a) pushover curve in X-direction response; (b) Pushover curve in Z-direction response

Figure 6-17. Wind pushover response of the 40-story building; nonlinearity distribution in near-collapse stage in the $\mathrm{X}$-direction response

Figure 6-18. Wind pushover response of the 40-story building; nonlinearity distribution in near-collapse stage in the Z-direction response.....

Figure 6-19. Wind directional dynamic response of the 40-story building: (a) peak drift story ratio; (b) peak base shear.

Figure 6-20. Wind directional dynamic story-response of the 40-story building: (a) peak drift story ratio in X-direction; (b) peak drift story ratio in Z-direction; (a) peak floor acceleration in X-direction; (b) peak floor acceleration in Z-direction

Figure 6-21. IDA responses of the 40-story building: (a)peak story drift ratio in X-direction; (b) peak story drift ratio in Z-direction; (c) peak floor acceleration in X-direction;

(d) peak floor acceleration in Z-direction 
Figure 7-1. Component acceptance criteria: (a) recommended by Griffis et al. (2012); (b) adopted and used in this study

Figure 7-2. Definition of Deformation Damage Index (DDI) for a given Drift Damage Zone (DDZ) in $\mathrm{n}^{\text {th }}$ story

Figure 7-3. Basic evaluation curves for wind-induced vibration (ISO 10137 (2004), Figure D.1)

Figure 7-4. Component performance assessment of the 47-sotry building for 120 and 135degree wind directions

Figure 7-5. Component performance assessment of the 47-sotry building for 150 and 165 degree wind directions.

Figure 7-6. Component performance assessment of the 47-sotry building for 180 and 195degree wind directions

Figure 7-7. General component performance assessment of the 47-story building based on all six critical wind directions

Figure 7-8. Interior and exterior drift damage zones (DDZs) in the 47-story building considered for cladding performance assessment.................................................. 206

Figure 7-9. Assessment of the performance of cladding system of the 47-story building to wind-induced shear deformation ...

Figure 7-10. Assessment of the serviceability performance of the 47 -story building related to

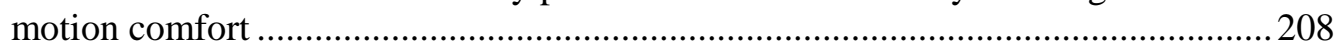

Figure 7-11.10-year MRI peak acceleration responses of the 47-story building in $\mathrm{X}$ and $\mathrm{Z}$ directions in respect to basic evaluation curves for wind-induced vibration.

Figure 7-12. Component performance assessment of the 30-story building for zero and 15degree wind directions

Figure 7-13. Component performance assessment of the 30-story building for 30 and 45degree wind directions

Figure 7-14. General component performance assessment of the 30-story building based on all four wind directions.

Figure 7-15. Interior and exterior drift damage zones (DDZs) in the 30-story building considered for cladding performance assessment.

Figure 7-16. Assessment of performance of cladding system of the 30-story building to wind-induced shear deformation .

Figure 7-17. Assessment of the serviceability performance of the 30 -story building related to motion comfort 
Figure 7-18. 10-year MRI peak acceleration responses of the 30-story building in X and Z directions in respect to basic evaluation curves for wind-induced vibration

Figure 7-19. Component performance assessment of the 40-story building for zero and 15 degree wind directions

Figure 7-20. Component performance assessment of the 40-story building for 30 and 45 degree wind directions.

Figure 7-21. General component performance assessment of the 40-story building based on all four wind directions....

Figure 7-22. Interior and exterior drift damage zones (DDZs) in the 40-story building considered for cladding performance assessment.......

Figure 7-23. Assessment of performance of cladding system of the 40-story building to wind-induced shear deformation .....

Figure 7-24. Assessment of the serviceability performance of the 40-story building related to motion comfort

Figure 7-25. 10-year MRI peak acceleration responses of the 40-story building in X and Z directions in respect to basic evaluation curves for wind-induced vibration. 


\section{ABBREVIATIONS AND ACRONYMS}

$\begin{array}{ll}\text { CP } & \text { Collapse Prevention } \\ \text { DDI } & \text { Deformation Damage Index } \\ \text { DDZ } & \text { Drift Damage Zone } \\ \text { EDP } & \text { Engineering Demand Parameter } \\ \text { FO } & \text { Fully Operational } \\ \text { IDA } & \text { Incremental Dynamic Analysis } \\ \text { IM } & \text { Intensity Measure } \\ \text { LO } & \text { Limited Operations } \\ \text { LS } & \text { Life Safety } \\ \text { PBWD } & \text { Performance-Based Wind Engineering }\end{array}$




\section{CHAPTER I}

\section{INTRODUCTION}

\subsection{Background}

Hurricanes are one of the most destructive forces in nature. Six out of ten costliest natural disasters in the history of the United States were caused by hurricanes. The United State has been struck by 284 hurricanes between the years 1851 to 2010, including 96 major hurricanes from categories $3,4 \& 5$. This is equivalent to about two major hurricanes every three years making landfall along the Mexican Gulf or Atlantic coast (NOAA, 2011). Table 1-1 shows the Saffir/Simpson (maximum 1-min wind at height of $33 \mathrm{ft}$. over unobstructed exposure) wind speed ranges corresponding to each hurricane category.

Table 1-1.The Saffir/Simpson hurricane wind scale (NOAA, 2011)

\begin{tabular}{|cc|}
\hline $\begin{array}{c}\text { Scale Number } \\
\text { (Category) }\end{array}$ & $\begin{array}{c}\text { Winds } \\
\text { Maximum 1-min } \\
(\mathrm{mph})\end{array}$ \\
\hline 1 & $74-95$ \\
2 & $96-110$ \\
3 & $111-130$ \\
4 & $131-155$ \\
5 & $>155$ \\
\hline
\end{tabular}

Figure 1-1 shows the tracks of the 30 costliest hurricanes in the United States between the years 1900 to 2010 . Several big cities with high-density high-rise areas can be located within the tracks. Many devastating storms, such as Sandy (2012), Katrina (2005), Andrew (1992), Alicia (1983), and others have struck major cities containing heavily occupied residential or commercial areas. The cities of New York, New Orleans, Miami, and Houston have been affected by such 
hurricanes, and many other major cities in the United States and around the world are at the risk of hurricanes and extreme storms.

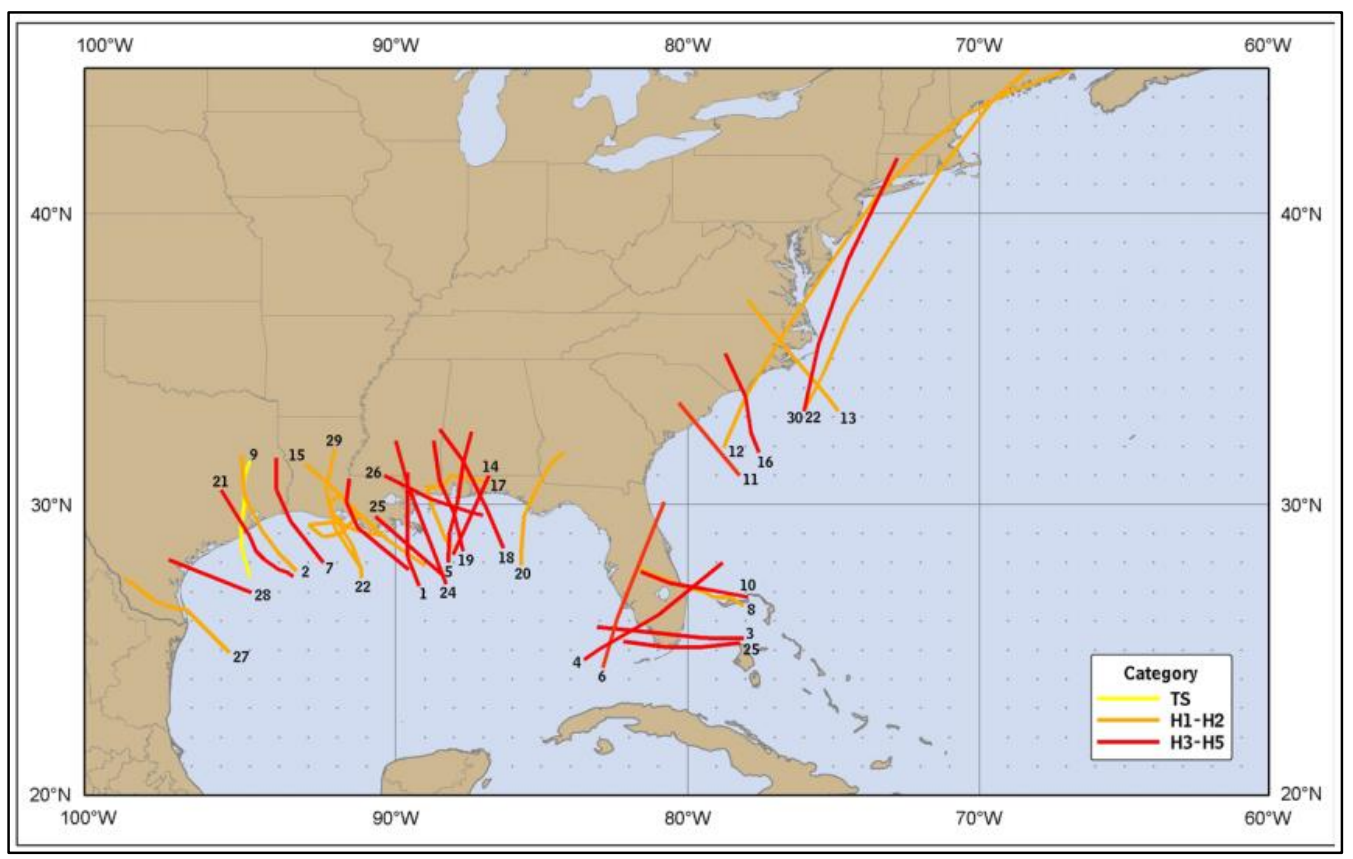

Figure 1-1.The 30 costliest tropical cyclones to strike the United States, 1900-2010 (NOAA, 2011)

Recent climate change studies indicate an increase in the destructiveness of hurricanes over the past 30 years (Emanuel, 2005; Goldenberg, Landsea, Mestas-Nunez, \& Gray, 2001; Webster, Holland, Curry, \& Chang, 2005). The destructiveness of a hurricane is measured based on the total dissipation power over its lifetime. The increase in destructiveness is due to the increase in both the lifetime and the intensity of the hurricanes. It is shown that over the North Atlantic and eastern and western North Pacific, the annual average storm peak speed and the duration of hurricanes have increased by $50 \%$ and $60 \%$ respectively in the past 30 years (Emanuel, 2005).

In addition, it has been observed that the coastal and near-coastal urbanization has increased in the last fifty years. During the period, about fifty million residents have moved to the coastal areas (Mishra, 2010). This increase in population and asset in hurricane-prone coastal areas causes lots of difficulties for the emergency management and increases the potential economic 
and human losses. Although modern facilities and improved emergency response systems decreased the number of death due to the hurricane, but the economic losses have increased significantly.

\subsection{Performance of High-Rise Buildings in Recent Major Hurricanes}

The study of the performance of high-rise buildings (defined as 10-story and taller buildings) in two recent major hurricanes of Sandy (2012) and Katrina (2005) indicated no structural damage to tall buildings in cities of New York and New Orleans (FEMA, 2013; Kareem \& Bashor, 2006). The observed damage was mainly to the cladding of the tall buildings, likely caused by windborne debris. Pea gravel, rooftop appurtenances, siding and penthouse structures \& etc. become airborne and formed wind-borne debris causing significant damage to the cladding of the surrounding buildings. In some high-rise buildings, the collapse of nonstructural partitioning walls and rooftop equipment was also reported during the hurricanes.

The evacuation of large populations of high-rise building residents poses a major risk to society and government. Recorded major wind events such as Hurricane Alicia in 1983, Hurricane Andrew in 1992, Hurricane Isabel in 2003, Hurricane Katrina in 2005 and Hurricane Sandy in 2012 have necessitated the evacuation of major cities, including many high-rise office and residential buildings. Indeed, one of the major lessons from Hurricane Rita in 2005 and Hurricane Ike in 2008 was the potentially disastrous consequence of evacuating a major metropolitan area like the city of Houston, causing widespread panic with large segments of the public becoming stranded on the congested highways, as shown in Figure 1-2, with no place to go for protection and riding out the hurricane in their cars, rather than being in safe shelters $(\mathrm{Li}$, Ozbay, \& Bartin, 2014; Lindsay, 2010). 


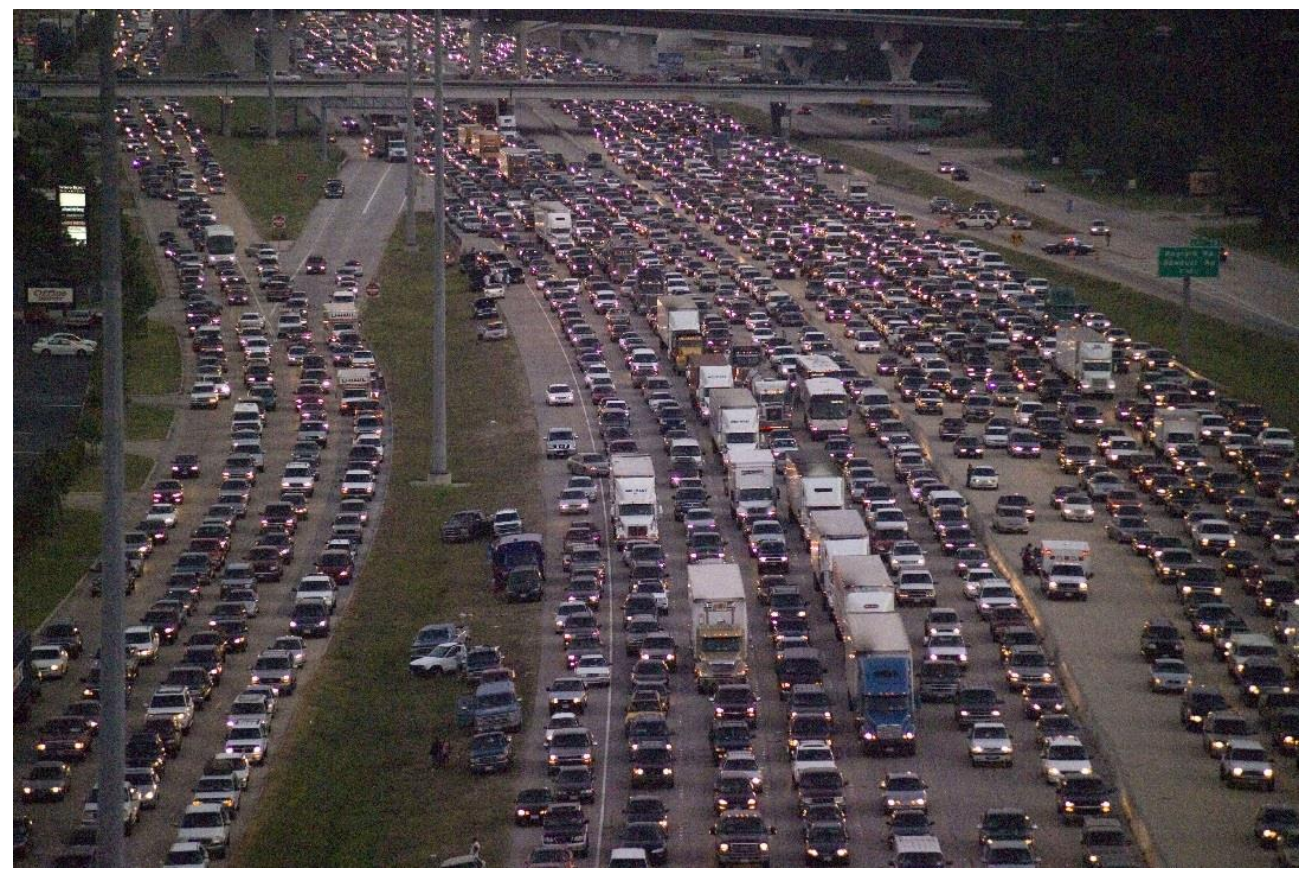

Figure 1-2. Traffic Jam during evacuation from Hurricane Rita, Houston, TX

Vertical evacuation is known as a preventative strategy characterized by vertical movement in order to stay above the hazard. Utilizing vertical evacuation structures, particularly high-rise buildings in condensed urban areas, has been considered as a key emergency response strategy for tsunamis. Design guidelines also have been developed for the designated vertical evacuation buildings (FEMA, 2012; Heintz \& Mahoney, 2008; Yeh, Robertson, \& Preuss, 2005). While areas exposed to hurricanes will be subjected to extreme wind loading and also most likely to flooding, scouring, surging and battering by water and airborne debris, vertical evacuation in hurricanes also has been considered as an emergency response. The motivation for the vertical evacuation in high-rise buildings during hurricanes is their reliable performance in past wind extreme events. Although these buildings experienced severe roof and cladding damage, as observed in Downtown Miami during Wilma Hurricane (2005), the collapse of the frames or the foundation has not been observed. Stubbs and Sikorsky (1987) performed a structural risk assessment to qualify the risk associated with vertical evacuation during a hurricane. The study 
suggested scenarios where the vertical evaluation is feasible, considering the feasibility criteria based on least risk and costs.

The investigation of the performance of high-rise buildings in New Orleans during hurricane Katrina indicated the possibility of the vertical evacuation in high-rise buildings during a hurricane. The investigation indicates two high-rise buildings, the Hyatt Regency Hotel and the Sheraton Hotel, refuged 3000 and 1000 people respectively including local residents, tourists and employees in interior regions of the buildings. The study suggests that the interior regions of high-rise buildings may be considered to serve as shelters to accommodate local residents during a hurricane. It can be advantageous in cities with limited escape routes and poor transportation. But it requires a pre-evaluation of the structural performance as well as the cladding performance of those selected high-rise building under extreme wind loads. The current design methodologies and codes failed to predict the damage caused by recent hurricanes such as Katrina (Gutierrez, Cresanti, \& Jeffrey, 2006). Performance-based engineering is the possible solution to probability assessment of direct and indirect damage to high-rise buildings due to extreme wind events.

\subsection{Current Wind Design of High-Rise Buildings}

Tall buildings, unlike low-rise buildings, are generally susceptible to dynamic excitations such as earthquake and wind. Figure 1-3 shows the reduced spectral density versus the frequency (period) for both wind and earthquake dynamic excitations. The figure indicates the distribution of energy corresponding to both wind and earthquake excitations as a function of frequency. As shown in the figure, for tall buildings (with a typical natural frequency less than $1 \mathrm{~Hz}$ ) the dynamic response characteristics are governed by the wind loading. Thus, the characteristics of dynamic wind loading and the corresponding building response are the major design concerns of tall buildings. 


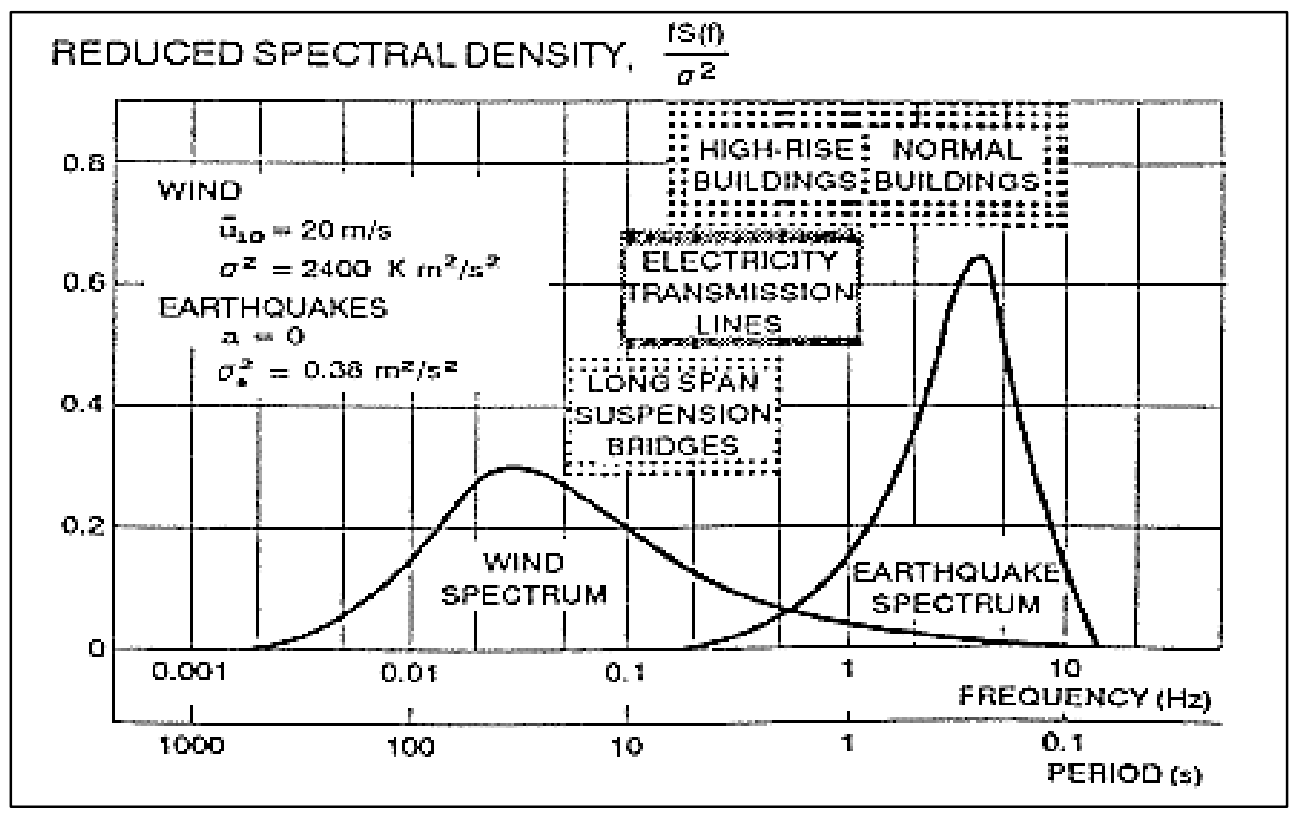

Figure 1-3. Energy distribution of earthquake and windstorm excitations in frequency domain (Borges, 1969)

In the current wind design approach as specified in ASCE 7-10 (ASCE, 2010), the structural members are designed (either using ASD or LRFD design approach) for the strength limit states which include an equivalent static wind loading corresponding to a specific hazard level determined based on the given risk category. For high-rise buildings, especially those with irregular geometry, the wind tunnel procedure is alternatively used to determine the design wind loads. This prescriptive approach does not provide transparent methods and criteria to reliably quantify the performance of buildings as well as the functional requirements necessary to accommodate large populations during extreme wind. In addition, current building design codes do not provide serviceability limit states enforcing story drift and perception to motion limiting requirements. Since this approach primarily intends to keep the structural system essentially elastic, a more efficient design may be achievable by allowing controlled inelastic behavior in structural components.

In the current design of high-rise buildings, the minimum wind load criteria of ASCE 7 are usually supplemented by additional design checks to control building motions in order to 
maintain human comfort under less extreme winds that occur more frequently than the design wind loads. These criteria vary depending on the building occupancy, but generally limit the maximum building accelerations to less than about 5 to 20 milli-g's for wind speeds with 1 to 10 years return periods. However, the current design approach does not provide requirements and limitations regarding the motion comfort for wind loads associated with higher wind hazard levels when the buildings are accommodating a large number of people in the extreme conditions of a major hurricane.

\subsection{Wind Performance-Based Design}

Performance-based engineering is a modern engineering process through which a new structure is designed or an existing building is evaluated and retrofitted more efficiently and economically by requiring the structure to meet certain performance requirements at various levels of demand. Comparing to the current prescriptive approach, utilizing the performance-based approach results in more informative and transparent output to stakeholders. The performance-based engineering concept was first developed and implemented in the seismic engineering field and is now well accepted in professional practice for seismic design and evaluation.

The inherent uncertainty in a building's response to the natural hazards of earthquake and wind causes the seismic and wind performance based design and evaluation to have a probabilistic basis. The Pacific Earthquake Engineering Research Center (PEER) proposed a probabilistic assessment framework for seismic performance based design as shown in Figure 1-4. As indicated in the figure, the framework consists of four analysis phases:

- Hazard analysis,

- Structural Analysis,

- Damage Analysis,

- Loss Analysis 
The first phase "hazard analysis" focuses on the probabilistic assessment of the hazard by developing a numerical probabilistic distribution function for the hazard at the specific location of the building. The outcome of this phase is a probabilistic assessment of the Intensity Measure (IM) where the Intensity Measure is an attribute of the hazard that is useful for predicting the damage or loss. For instance, peak ground acceleration or spectral acceleration is normally used as IM in seismic evaluations. Typically, the IM is determined as a mean probability of recurrence. For wind, the basic wind speed, as defined in ASCE 7, may be used as the intensity measure in the wind performance-based design approach. This phase also involves providing appropriate hazard input records, such as ground motion acceleration and pressure/force time-history records for seismic and wind events respectively.

The next phase involves performing structural analyses for different IM levels using the input loading time-history records obtained from the previous phase. The outcome of this phase will be Engineering Demand Parameters (EDP) which are terms of structural responses properly correlated to the corresponding structural and non-structural damage. In Seismic analysis, the most common EDPs for buildings are inter-story drift ratios and floor acceleration spectra.

The next phase in the framework is to perform a damage analysis to relate the EDPs to Damage Measures (DM). Damage Measures describe the physical damage (structural and non-structural) to the building, to qualify the damage to the life safety and functionality of the building as well as the required repairs. The outcome of this phase, integrated with EDP probability obtained in the previous phase, represents the annual probability of exceedance for the DMs.

The final phase of the process is to calculate Decision Variables (DV). Generally, DVs are determined according to three decision metrics: (1) direct Dollar loss, (2) restoration time and (3) casualties. The DVs facilitate the decision making by stakeholders on cost-effective risk management of the built environment subjected to either seismic or wind hazard. 


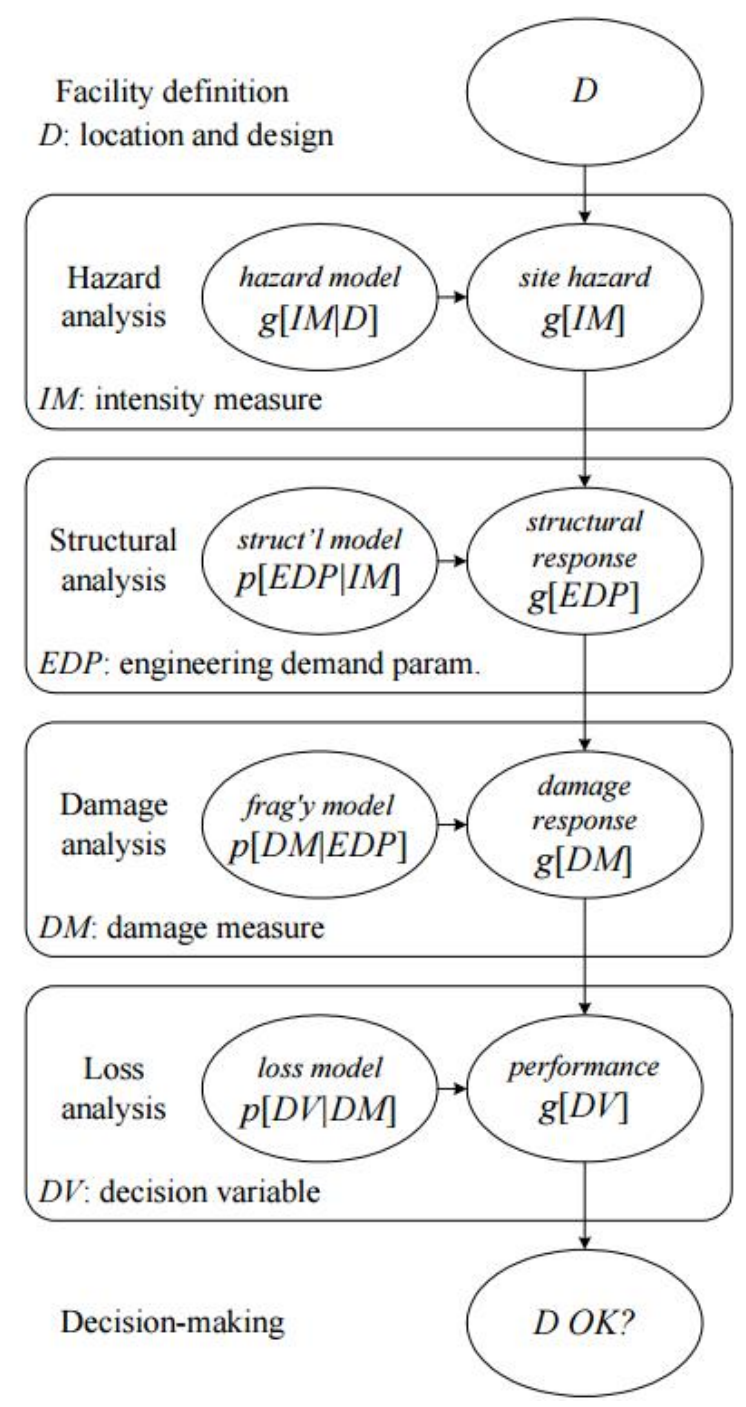

Figure 1-4. PEER's framework for seismic performance-based design (Porter, 2003)

The general performance based engineering approach as described above first advanced in practice for the seismic evaluation of existing buildings by a framework introduced in SEAOC Vision (2000), enhanced in successor guidelines ATC40(ATC, 1996), FEMA-273/274(FEMA, 1997a), FEMA-356 (FEMA, 2000a), ASCE41-06 (ASCE, 2007) and ASCE41-13 (ASCE, 2014). The framework correlated the performances evaluated at discrete levels of seismic hazard to expected performance objectives representing a certain state of functionality, damage and corresponding loss of the building. Figure 1-5 shows the recommended seismic framework including the performance assessment in four levels of hazard associated with earthquakes 
represented in terms of the Mean Recurrence Interval (MRI). Four levels of performance objectives including Fully Operational, Operational, Life Safety and Near Collapse are specified for three importance levels of Basic Objectives, Essential Objectives and Safety Critical Objective. According to the framework, for the Basic Objective level, it is required to meet objective performances of Fully Operational, Operational, Life Safety and Near Collapse in Frequent, Occasional, Rare and Very Rare hazard levels respectively.

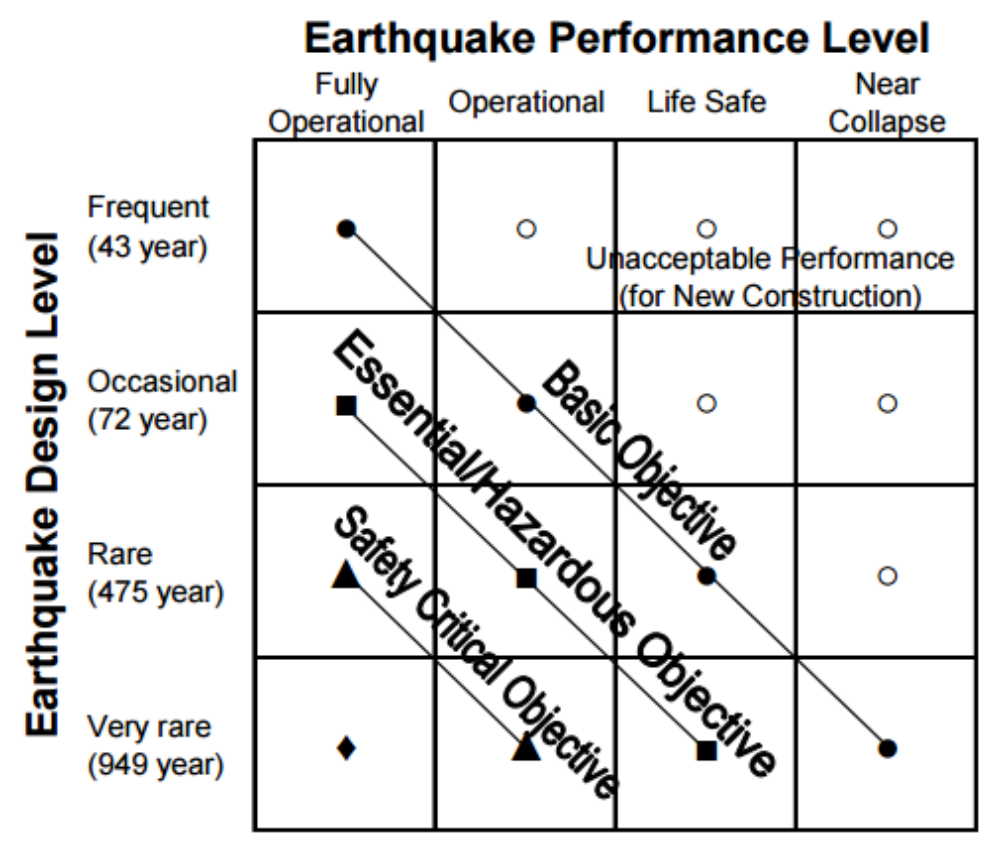

Figure 1-5. Recommended seismic performance objectives (SEAOC, 2000)

Using the performance-based approach leads to more efficient and economical design of new buildings and more transparent evaluation of existing buildings by requiring the structure to meet certain performance requirements at various levels of the demand. Compared to the current prescriptive approach, utilizing the performance -based approach results in more informative and transparent output to stakeholders. Performance-based engineering concepts were first implemented and developed in seismic engineering field and are now well accepted in professional practice for seismic design and evaluation. 
Modern Performance based design approach for a given building may include following sequences:

1) Setting hazard and performance levels: Hazard levels typically characterized with occurrence probability are to be set. Different performance levels associated with specific hazard levels then need to be specified. The performance requirements generally involve metrics reflecting: the money losses associated with repair and restoration; loss of functionality; and the risk of casualties.

2) Modeling the building and analyzing it for certain hazard levels and obtaining demand parameter responses: In addition to a component's demand parameters, lateral displacement, velocity and acceleration response of the building may provide insight into its overall performance. Story drift ratio response illustrates the distribution of lateral displacement over the height of the building, as well as providing a measure of performance of deformation-sensitive non-structural elements such as facade, interior partitions etc. Peak floor acceleration may be used to evaluate the motion comfort serviceability under wind excitation.

\section{3) Check the performance by comparing demand parameters and the respective acceptance}

criteria: The performance acceptance criteria are specified for components and overall system responses of the building. They are distinguished based on the component type; either deformation-controlled (ductile component, expected to develop significant inelastic deformation, the capacity governed by the deformation) or force-controlled (non-ductile component, expected to experience insignificant inelastic deformation, the capacity governed by the strength). The acceptance criteria may depend on the type of the analysis implemented either nonlinear static or dynamic analyses. The difference in acceptance criteria accounts for the facts related to the realistic dynamic response which is not considered in static nonlinear analysis such as cyclic degradation. The component models for static nonlinear analysis do not include the cyclic 
strength and stiffness degradations. The comparison between demand parameter values and acceptance criteria are usually expressed by demand-capacity ratios.

Recently, the application of performance-based engineering has been extended to other fields, particularly in wind engineering. Some wind performance based design frameworks have been suggested in the last decade (Bartoli, Ricciardelli, Saetta, \& Sepe, 2006; Ciampoli, Petrini, \& Augusti, 2011; Griffis, Patel, Muthukumar, \& Baldava, 2012; Petrini, 2009). The use of performance-based design in wind engineering was motivated by the fact that the current wind design excludes the development of any nonlinearity in structural members. Disregarding the nonlinear capacity of the structure tends to lead to a conservative design. The wind performance based design approach enables the designers to design buildings efficiently according to the desired performances in various hazard levels. Figure 1-6 shows a wind performance-based framework suggested by Griffis et .al (2012). The framework is comparable to recommended framework for seismic engineering shown in Figure 1-5, except the fact that relatively moderate levels of performance are generally considered for the wind performance-based engineering framework. For instance, no near collapse performance level is considered for response to wind as shown in the figure. It might generally be referred to the different natures of wind and earthquake events and their time duration. It may suggest that the serviceability performances are the governing criteria in performance-based wind design. 


\begin{tabular}{|c|c|c|l|l|}
\hline \multirow{2}{*}{$\begin{array}{l}\text { Wind } \\
\text { Hazard } \\
\text { (MRI) }\end{array}$} & \multicolumn{3}{|c|}{ Performance Objectives } \\
\cline { 2 - 5 } & $\begin{array}{c}\text { Motion } \\
\text { comfort }\end{array}$ & $\begin{array}{l}\text { Continued } \\
\text { occupancy }\end{array}$ & Operational & $\begin{array}{l}\text { Limited } \\
\text { interruption }\end{array}$ \\
\hline 1 year & & & & \\
\hline 10 years & & & \\
\hline 50 years & & & \\
\hline 100 years & & & \\
\hline 700 years & & & \\
\hline 1700 years & & & \\
\hline
\end{tabular}

Figure 1-6. Wind Performance objective matrix (Griffis et al., 2012)

\subsection{Problem Statement}

At the present point in time the following facts point to a problem in need of a solution: (1) the fast growth of high-rise high-density urban areas in coastal cities; (2) the potentially disastrous transportation chaos associated with evacuating high-rise urban areas; (3) the significant increase in the hazard associated with wind events, especially hurricanes, due to climatic changes in last few decades; and (4) the deficiencies of the current prescriptive wind design approach in considering structural nonlinearity and providing appropriate functionality and performance requirements to accommodate a large number of people during hurricanes. These facts bring up the following questions and concerns:

1. How will existing and new high-rise building respond to increasing wind hazard due to recent climate change? 
2. How will the significant inelastic capacity of the structural components, which is disregarded in the current wind design approach, help in developing high-rise buildings that are resilient to future severe wind events?

3. How will the consideration of inelasticity of structural components in wind design affect serviceability and non-structural performance of the building?

4. Can new and existing buildings be designed or modified to be suitable shelters during extreme wind events?

5. How can the performance-based engineering approach, which is now well developed and in use in seismic engineering, address the above questions and concerns?

\subsection{Research Objectives}

The objectives of this research are to: (1) numerically investigate the characteristics of uncertain nonlinear dynamic response of high-rise buildings to extreme wind events; (2) experimentally acquire wind loading data and studying the effect of building's realistic environment (such as the building's shape characteristics and the effects of the surrounding building) on the dynamic wind loading characteristics for an existing high-rise building; and (3) develop and implement a performance-based engineering procedure to assess the performances of high-rise building models to different hazard levels associated with wind excitations.

The objectives are achieved by accomplishing following tasks:

- Studying the nonlinear dynamic response of high-rise buildings through:

- Gathering the information related to high-rise buildings to be studied by conducting wind dynamic analyses.

- Developing a reliable analytical model to conduct nonlinear wind response history analyses. 
- Adopting state-of-the-art reliable methods to accurately consider the nonlinearity of structural members in large complex analytical models of high-rise buildings.

- A study on the dynamic response analysis assumptions such as damping and validating that the analytical approach reflects them correctly.

○ Conducting verification analyses using available numerical and experimental results to verify the reliability of the developed analytical approach.

- Conducting sets of wind pressure testing to obtain the realistic wind loading on the existing high-rise building and also investigating the interference effect of neighboring buildings and buildings shape characteristics.

- Developing a numerical approach to apply measured wind loading to an analytical model with highest achievable accuracy.

- Studying the directionality characteristics of the wind response by repeating wind dynamic analyses for different wind directions around the building for a constant wind speed.

- Studying the wind nonlinear dynamic response of the high-rise building model by implementing an Incremental Dynamic Analysis approach (IDA).

- Developing a wind performance assessment approach by adopting reliable performancebased wind design criteria available in the literature.

- Conducting wind performance assessment of the high-rise models considering different types of performances and discussing the obtained results.

- Developing a set of recommendations based on the obtained performance assessment results. 


\subsection{Research Approach and Methodology}

The nonlinear dynamic responses of three steel moment frame high-rise buildings were identified by developing 3D nonlinear finite element models using OpenSEES finite element software. Different types of structural component nonlinearity (including steel beams and column plastic hinging, column panel zone yielding, nonlinear response of both moment resisting and gravity connections) were incorporated into the finite element models using state-of-the-art approaches mainly developed in seismic engineering field. The analytical approach was then verified in different stages by simulating both proven analytical models and experimental data available in the literature. Wind dynamic loading was acquired by either conducting sets of wind pressure testing or using available wind tunnel data. The dynamic wind loads on high-rise building models were simulated by a large number of equivalent time-history point loads to achieve the highest possible accuracy.

The wind dynamic analyses were performed for different wind directions to study the directionality characteristics of the wind dynamic response. An incremental dynamic analysis (IDA) approach was applied to investigate the response of the high-rise buildings to incremental wind hazard levels represented by corresponding basic wind speeds. The responses of the structural components in addition to the global responses including the story drift ratios and floor accelerations for all IDAs were recorded and analyzed to be used in the wind performance assessments.

The wind performance assessments of the high-rise building models were performed by adopting wind performance-based design criteria recommended in the literature. A performance assessment approach evaluating three types of performance including structural component performance, cladding performance to wind induced inter-story shear deformation and serviceability motion comfort performance, was developed. The IDA results along with adopted 
acceptance criteria were utilized to estimate the expected performance levels of the buildings for the different wind hazard levels represented in terms of associated basic wind speed.

The performance assessment results for all three high-rise building models were discussed in relation to their design characteristics. The lessons learned from these case study wind performance based evaluations were presented in terms of a set of recommendations.

\subsection{Organization of Dissertation}

This dissertation is organized in eight chapters. Chapter 1 provides an introduction to increasing hazard associated with extreme wind events especially hurricane impacts on high-density highrise urban areas. The observed increasing trend in the destructiveness of hurricanes and the consequent risks facing coastal cities in recent decades are discussed. This chapter also reviews the deficiencies of current wind design approach related to not providing resilience and functionality performance requirements and disregarding the inelastic lateral capacity of the buildings. This chapter also reviews the current performance-based engineering approach and its capability to address the mentioned concerns. The chapter concludes with explaining the research objectives and methodology.

Chapter 2 first introduces three high-rise buildings studied in this research and then discusses the analytical approach implemented to simulate the nonlinear dynamic response of buildings subjected to wind excitations. The chapter explains the methodology used to develop a 3D nonlinear analytical model by reviewing state-of-the-art methods developed for simulating nonlinearity in the structural members. The chapter describes approaches to properly consider cyclic response characteristics of the structural components, essential in the dynamic response simulations. The different types of cyclic deteriorations and their incorporation in available analytical models are broadly discussed. The chapter discusses the analytical approaches to simulate beam and column nonlinearity, panel zone yielding, nonlinear response of pre- 
Northridge connections and shear tab gravity connections. It explains the numerical methods either adopted from the literature or developed in this study, with respect to the available experimental data. It also represents the investigations conducted to identify the significance of the component shear deformations and geometric nonlinearity effects on the lateral response of a high-rise building. The chapter concludes by summarizing the developed analytical approach.

Chapter 3 explains the verification analyses conducted in the different stages of this study to validate the reliability of the developed analytical approach and finite element solution. It presents a few analytical studies in the literature which were simulated using the developed analytical approach to validate it through comparing the obtained results. It also presents a further round of validations conducted by modeling and analysis of two full-scale shake table tests.

Chapter 4 describes the investigation conducted on the modeling of damping in the analytical approach. The chapter reviews recent research indicating the issues related to using Rayleigh damping for dynamic analysis of nonlinear systems and the suggested solutions to avoid spurious damping forces. It explains the investigation conducted by studying the free vibration of elastic and inelastic systems and the solution implemented in this research to guarantee reliable modeling of damping in the analytical approach.

Chapter 5 describes the aerodynamic boundary layer wind pressure tests carried out on a scaled high-rise building model in the Wall of Wind (WOW) facility at Florida International University. The characteristics of the simulated wind flow and details of scaled model are provided. The obtained results for the three sets of tests are presented and discussed. The chapter provides a comparison between WOW wind test results and wind tunnel data provided by TPU Aerodynamic database for high-rise buildings. It also explains the wind tunnel data acquired from TPU Aerodynamic database for two high-rise building models. An explanation of the 
procedure used to convert measured wind pressure data to time-histories point loads applicable into the analytical model concludes this chapter.

Chapter 6 presents the analytical results obtained for the three high-rise models. Separately for each building, it first presents the modal analysis results. The wind nonlinear pushover results and the estimated wind over-strength is then presented. This chapter discusses the wind directionality dynamic response characteristics of a high-rise building. It then explains the IDA approach implemented in this study and discusses the obtained IDA results.

Chapter 7 explains the performance assessment approach developed in this study along with the adopted wind performance criteria. It presents wind performance assessments of all three highrise buildings. The estimated performances for different levels of wind loading are then presented and discussed. The estimated performances are also compared with performance objectives recommended by an available wind performance-based design framework.

Chapter 8 provides a summary and the conclusions of the conducted research and the wind performance-based assessment approach developed within this study. The estimated performance levels of the three buildings are discussed with respect to their wind design characteristics. The chapter summarizes the conclusions, recommendations and suggestions for future studies. 


\section{CHAPTER II}

\section{DEVELOPMENT OF THE ANALYTICAL PROCEDURE}

\subsection{Background}

Current wind design procedures usually require an elastic static analysis to check that the structural elements remain in the elastic range for the given static wind load pattern. However, modern performance-based design procedure requires determining the realistic behavior of the building for different levels of wind loads (corresponding to different MRIs) using nonlinear response history analyses. Advancements in computing technologies as well as available experimental data and the developed numerical approaches in seismic studies have enabled conducting nonlinear time history analyses of large high-rise buildings. While there are wellestablished elastic analysis approaches, nonlinear analysis approaches and techniques are still developing and improving. There are still limitations and a lack of experimental support associated with available nonlinear analysis approaches, particularly for 3D analyses. Performing nonlinear response history analysis involves two types of significant challenge: (1) the challenges associated with considering nonlinearity in structural elements, such as the modeling of nonlinearity (distributed or concentrated), and the consideration of strength and stiffness deterioration related to inelastic material behavior (which is discussed in this chapter); and (2) challenges associated with transient dynamic analysis such as the modeling of damping of buildings, discussed and investigated in next chapter.

The first widespread application of nonlinear analysis was in the late 1990s for the seismic evaluation and retrofitting of existing buildings. The seismic evaluations were proposed in terms of nonlinear static pushover analyses according to pioneer guidelines: ATC 40 Seismic Evaluation and Retrofitting of Concrete Buildings (ATC, 1996b) and FEMA 273 NEHRP Guidelines for the Seismic Rehabilitation of Buildings (FEMA, 1997b). At the same time, the 
nonlinear analysis was introduced to be used in seismic risk assessment to develop the seismic damage fragility functions (Kircher, Nassar, Kustu, \& Holmes, 1997; Kircher, Reitherman, Whitman, \& Arnold, 1997). Using improvements in computing technologies and experimental data on nonlinear response of structural components and numerical solutions, nonlinear analysis approaches were then proposed in FEMA 440 Improvement of Nonlinear Static Seismic Analysis Procedures (FEMA, 2005), ASCE 7-10 Seismic Rehabilitation of Existing Buildings (ASCE, 2007) and FEMA P440A Effects of Strength and Stiffness Degradation on Seismic Response (FEMA, 2009).

For more accurate performance assessment and design of buildings subject to dynamic excitations such as earthquake, wind, fire and explosion, nonlinear dynamic analysis has recently been widely utilized. The performance-based engineering has been particularly utilized for seismic evaluation and design of high-rise buildings in high seismic regions, where the lateral resisting system is not included in ASCE 7-10, Table 12.2-1. Recently, some guidelines have been developed, recommending nonlinear dynamic analysis for seismic evaluation and design for highrise buildings such as: PEER seismic Design Guidelines for Tall Buildings (PEER 2010), Recommendations for the Seismic Design of High-rise Buildings (Willford et al. 2008), and PEER/ATC 72-1 Modeling and Acceptance Criteria for Seismic Design and Analysis of Tall Buildings (PEER/ATC 2010). Development of fragility models relating structural demand parameters to explicit damage and loss metrics, employed with using nonlinear dynamic analysis lead to enhanced seismic performance assessment of new and existing buildings as recommended in ATC 58 Guidelines for Seismic Performance Assessment of Buildings (ATC, 2009).

By reviewing all state-of-the-art methods and approaches available for conducting nonlinear dynamic analyses, an analytical approach is established here to conduct nonlinear dynamic simulations to study the response of three high-rise building models under different levels of wind 
hazard. The three high-rise building are first described in the following section and then the developed analytical approach is broadly explained and discussed.

\subsection{Description of High-Rise Building Models}

\subsubsection{7-Story High-Rise Building Model}

The 47-story case study building investigated in this research is an office building located in the heart of downtown, Houston, Texas, surrounded by tall buildings with generally similar heights. The building has 47 above ground and 4 underground levels with typical 13' story height, the overall dimensions being $155^{\prime}-2$ " square and approximately $628 \mathrm{ft}$. above grade to the top of the parapet. The building contains a sloping glass "skirt" at its base which is approximately 213' square at its base and rises to meet the prismatic tower at approximately 60 feet above the ground. The building consists of perimeter steel moment-resisting frames and interior steel gravity framing and a concrete deck-steel beam composite roofing system. The perimeter frames constitute the only lateral load resisting system of the building. The building was designed in early 1971 according to the Houston Building Code which included the 1969 AISC specifications for the design, fabrication, and erection of steel structures. It was built in late 1971 and early 1972 and occupied in late 1972 to early 1973. It was then strengthened and renewed in 1994 by removing the skirt and adding a six-story cap to the building with a big hole cut into it. The reason for being interested in studying this building was that, in its older configuration and before the renovation, it survived Hurricane Alicia in 1983 without any significant structural damage. It was surprising since the building was designed based on an old code with primitive wind design criteria and would be considered underdesigned according to current wind design criteria.

Figure 2-1a and Figure 2-1b show the typical floor framing plan and perspective view of the 47story building respectively. Four almost identical perimeter moment resisting frames (shown in blue in the figure) consisted of five bays with around 30' spacing. The perimeter frames contain a 
total of 20 columns. The four corner columns consisted of 42"x42"x20" L-shaped box sections of 4" wall thickness in the lowest levels; and 14"x12" box sections of 4 " wall thickness in the highest levels. The sixteen middle columns consisted of 42 "x20" box sections of 31/2" wall thickness in the underground and lower levels, changing to $\mathrm{W} 14 \times 500$ and varying to $\mathrm{W} 14 \times 34$ in the highest levels. Girders in perimeter frames were built-up plate girders with 52" depth in the lower levels varying to 34 " depth in the upper levels, and nominally W24 beams in the top six levels. The gravity framing (shown in green and gray in Figure 2-1) includes versatile roofing beams averagely represented by $\mathrm{W} 18 \mathrm{x} 40$ beams. It includes 22 columns consisting of versatile W-14 columns averagely ranging from heavy W-section columns (e.g. W14x426) in the lower levels to light columns (e.g. W14x16) in the highest level.

All structural steel used in this building is Grade 50 steel. The typical floor slab consists of 51/4" thickness lightweight concrete with 2-inch corrugated metal deck. The expected gravity loads considered in the wind analyses consisted of 57 psf steel structure and concrete deck, 15 psf exterior walls and 15 psf superimposed loads including partitions, ceiling, mechanicals, and expected permanent live loads. It should be noticed that, among all gravity beams, only floor beams connecting two interior gravity columns or an interior gravity column and an exterior moment frame column (shown in green in Figure 2-1) are included in numerical model and other secondary beams were considered to collaborate to make a rigid diaphragm system with insignificant contribution to lateral stiffness and resistance. 


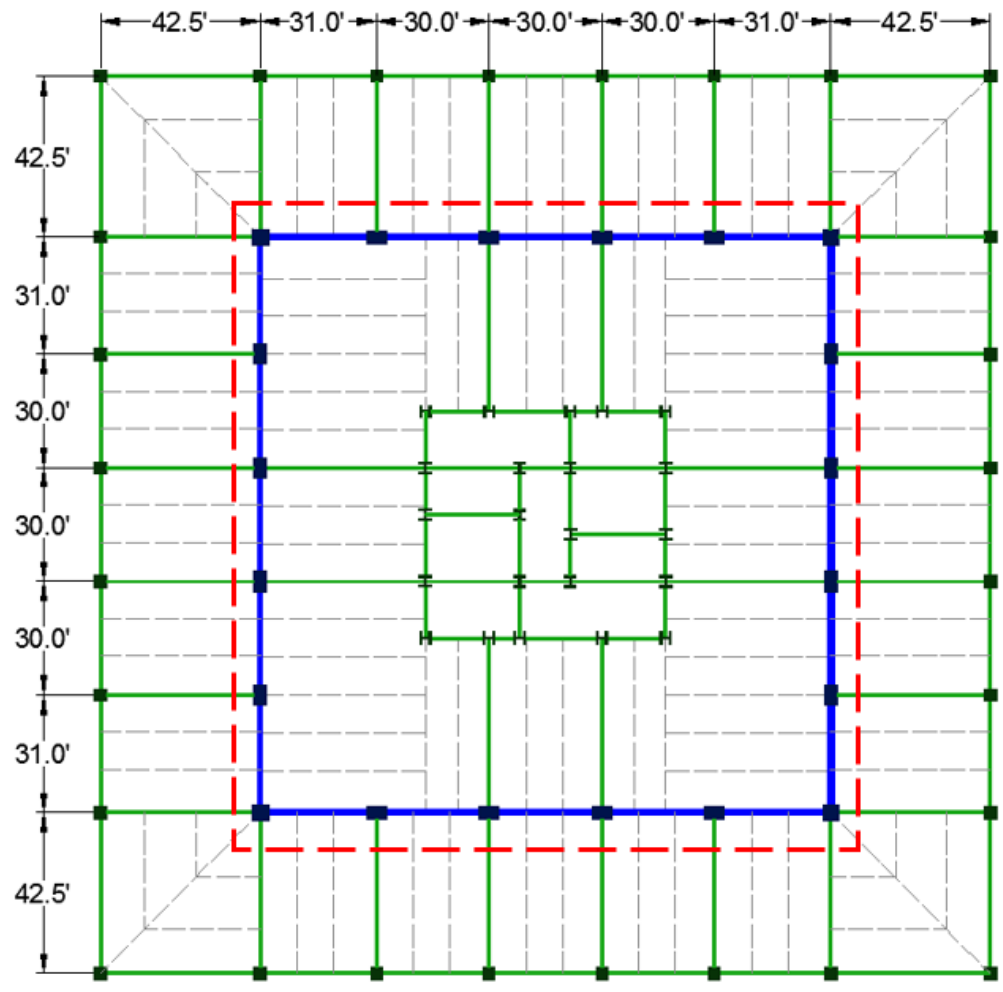

(a)

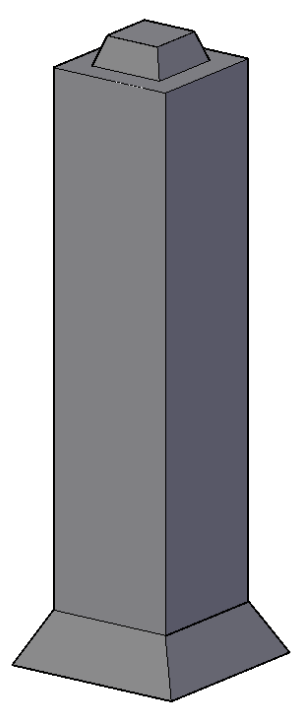

(b)

Figure 2-1. The 47-story building; (a) typical floor framing of the underground levels (the aboveground levels shown by the dotted red square); (b) perspective view of the building's shape before the renovation.

\subsubsection{0-Story and 40-Story Steel Moment High-Rise Building Models}

In addition to the existing steel moment frame high-rise building explained in the previous section, two steel moment frame high-rise building models from the literature were selected and investigated in this study. It was intended to conclude some general trends for wind performance of steel moment-frame high-rise building through the study of the three mentioned buildings.

An aspect of steel moment frame high-rise building studies is that the literature mainly focuses on seismic studies of the building designed for moderate and high seismic hazard areas, where essentially only Special Moment Frame (SMF) buildings are allowed. Use of SMF buildings for high-wind areas with the typically low seismic hazard is not a common practice. Considering these facts, two steel Intermediate Moment Frame (IMF) high-rise buildings, in tune with more 
common practice in high-wind and hurricane-prone areas, were selected and investigated for wind performance based evaluations.

Two 30-story and 40-story steel IMF high-rise building as designed and presented by Han, Moon, and Ha (2015) were adopted and investigated for wind loads through a performance-based evaluation approach. The high-rise building models are office buildings with dead and live design loads equal to $86 \mathrm{psf}$ and $20 \mathrm{psf}$ respectively. The buildings are designed for $\mathrm{C}$ category as Seismic Design Category (SDC) according to ASCE 7-10 using the following minimum design spectral acceleration parameters: short period $S_{D S}=0.33 \mathrm{~g}$; and 1-second period $S_{D 1}=0.133 \mathrm{~g}$. The buildings also were designed for a basic wind speed of $115 \mathrm{mph}$ and suburban terrain $\mathrm{B}$. Considering the above assumptions the buildings were designed according AISC 341-10 (2010a) and AISC 360-10 (AISC, 2010b). The lateral resistance of the 30-story building was designed with dominant seismic loads, although wind loads governed the design of the 40-story building. Figure 2-2and Figure 2-3 show floor plan, elevation and framing information for the 30-story and 40-story buildings respectively.

As shown in the figures the details of gravity beams and columns were not provided. For consistency of the analytical approach, gravity framing was incorporated by considering gravity columns equal to exterior column type (as shown in the figures) and W18x40 beams and corresponding semi-rigid shear tab gravity connections. Finite element models were then created for all three building models using methods and concepts explained in following sections. 


\section{$\mapsto$ Exterior column}

H Interior column

Tube section column

Moment Frames

- - Gravity Frames

- Pin connections

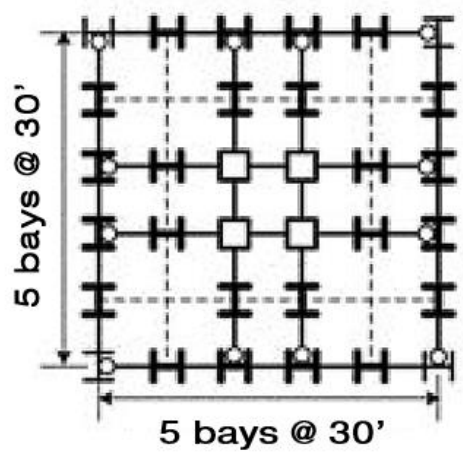

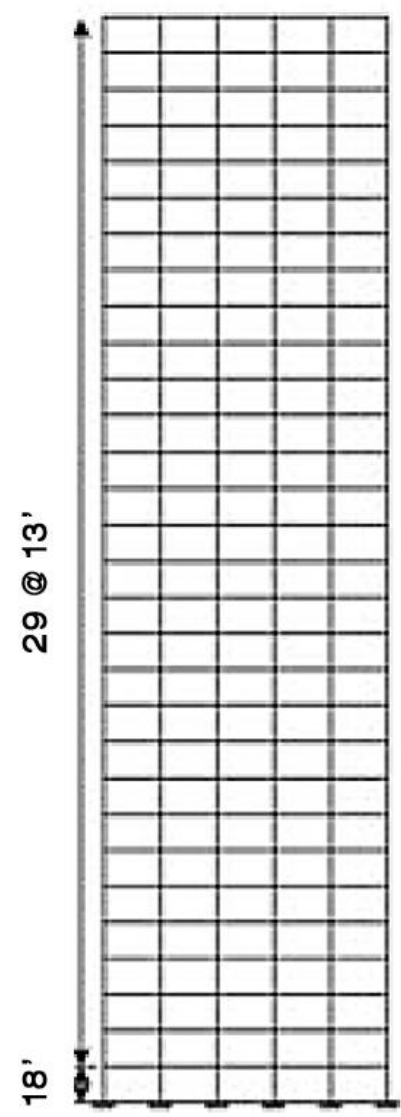

\begin{tabular}{|c|c|c|c|c|}
\hline \multirow{2}{*}{ Story / Floor } & \multicolumn{3}{|c|}{ Columns } & \multirow{2}{*}{ Girder } \\
\hline & Exterior & Interior & Tube section & \\
\hline $1 / 2$ & W14X665 & W14X808 & TS30x30×5/8 & W36X232 \\
\hline $2 \sim 3 / 3 \sim 4$ & W14X398 & W14X605 & TS $30 \times 30 \times 5 / 8$ & W36X232 \\
\hline $4 \sim 5 / 5 \sim 6$ & W14X342 & W14X550 & TS $28 \times 28 \times 5 / 8$ & W36X210 \\
\hline $6 \sim 7 / 7 \sim 8$ & W14X283 & W14X500 & TS $28 \times 28 \times 5 / 8$ & W36X182 \\
\hline $8 \sim 9 / 9 \sim 10$ & W14X257 & W14X455 & TS $28 \times 28 \times 5 / 8$ & W36X170 \\
\hline $10 \sim 11 / 11 \sim 12$ & W14X211 & W14X455 & TS $28 \times 28 \times 5 / 8$ & W36X182 \\
\hline $12 \sim 13 / 13 \sim 14$ & W14X159 & W14X398 & TS $28 \times 28 \times 5 / 8$ & W36X150 \\
\hline $14 \sim 15 / 15 \sim 16$ & W14X132 & W14X342 & TS $28 \times 28 \times 5 / 8$ & W36X150 \\
\hline $16 \sim 17 / 17 \sim 18$ & W14X109 & W14X283 & TS $26 \times 26 \times 5 / 8$ & W36X135 \\
\hline $18 \sim 19 / 19 \sim 20$ & W14X82 & W14X257 & TS $26 \times 26 \times 5 / 8$ & W $33 \times 130$ \\
\hline $20 \sim 21 / 21 \sim 22$ & W14X82 & W14X233 & $\mathrm{TS} 26 \times 26 \times 5 / 8$ & W30X108 \\
\hline $22 \sim 23 / 23 \sim 24$ & W14X74 & W14X193 & TS $18 \times 18 \times 5 / 8$ & W30X90 \\
\hline $24 \sim 25 / 25 \sim 26$ & W14X53 & W14X159 & TS $18 \times 18 \times 5 / 8$ & W27X84 \\
\hline $26 \sim 27 / 27 \sim 28$ & W14X43 & W14X109 & TS $16 \times 16 \times 5 / 8$ & W24X62 \\
\hline $28 \sim 29 / 29 \sim 30$ & W14X30 & W14X74 & TS $16 \times 16 \times 3 / 8$ & W21X44 \\
\hline $30 /$ Roof & W14X22 & W14X34 & TS16x4x1/2 & W14X26 \\
\hline
\end{tabular}

Figure 2-2. Floor plan, elevations and beam and column sections of the 30-story building (Han et al., 2015) 


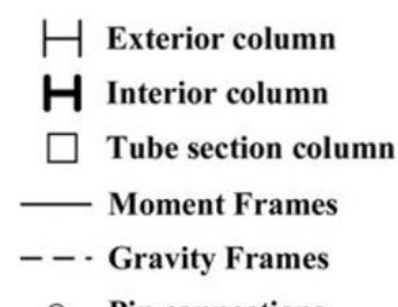

○ Pin connections
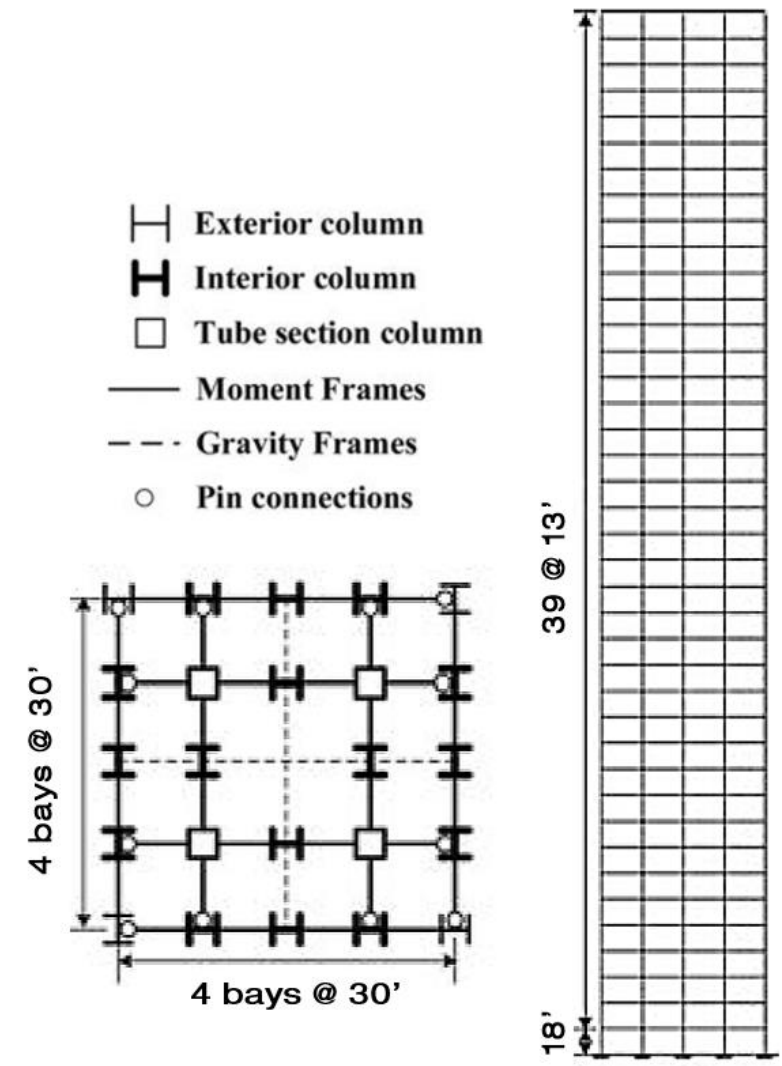

\begin{tabular}{|c|c|c|c|c|}
\hline \multirow{2}{*}{ Story / Floor } & \multicolumn{3}{|c|}{ Columns } & \multirow{2}{*}{ Girder } \\
\hline & Exterior & Interior & Tube section & \\
\hline $1 / 2$ & "W14X808 & W36X798 & 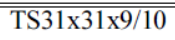 & W36X256 \\
\hline $2 \sim 3 / 3 \sim 4$ & W14X730 & W14X665 & TS $31 \times 31 \times 9 / 10$ & W36X256 \\
\hline $4 \sim 5 / 5 \sim 6$ & W14X605 & W14X730 & TS $31 \times 31 \times 4 / 5$ & W36X232 \\
\hline $6 \sim 7 / 7 \sim 8$ & W14X550 & W14X605 & $\mathrm{TS} 31 \times 31 \times 7 / 10$ & W36X232 \\
\hline $8 \sim 9 / 9 \sim 10$ & W14X500 & W14X550 & $\mathrm{TS} 31 \times 31 \times 7 / 10$ & W36X232 \\
\hline $10 \sim 11 / 11 \sim 12$ & W14X455 & W14X500 & $\mathrm{TS} 31 \times 31 \times 7 / 10$ & W36X232 \\
\hline $12 \sim 13 / 13 \sim 14$ & W14X370 & W14X500 & $\mathrm{TS} 31 \times 31 \times 7 / 10$ & W36X232 \\
\hline $14 \sim 15 / 15 \sim 16$ & W14X283 & W14X455 & $\mathrm{TS} 31 \times 31 \times 7 / 10$ & W36X230 \\
\hline $16 \sim 17 / 17 \sim 18$ & W14X257 & W14X398 & $\mathrm{TS} 31 \times 31 \times 7 / 10$ & W36X230 \\
\hline $18 \sim 19 / 19 \sim 20$ & W14X233 & W14X398 & TS $30 \times 30 \times 5 / 8$ & W36X230 \\
\hline $20 \sim 21 / 21 \sim 22$ & W14X193 & W14X342 & TS $30 \times 30 \times 5 / 8$ & W36X210 \\
\hline $22 \sim 23 / 23 \sim 24$ & W14X159 & W14X342 & TS $28 \times 28 \times 5 / 8$ & W36X194 \\
\hline $24 \sim 25 / 25 \sim 26$ & W14X145 & W14X257 & TS $28 \times 28 \times 5 / 8$ & W36X170 \\
\hline $26 \sim 27 / 27 \sim 28$ & W14X132 & W14X233 & TS $26 \times 26 \times 5 / 8$ & W36X170 \\
\hline $28 \sim 29 / 29 \sim 30$ & W36X170 & W14X176 & $\mathrm{TS} 24 \times 24 \times 5 / 8$ & W36X170 \\
\hline $30 \sim 31 / 31 \sim 32$ & W36X170 & W14X159 & TS $24 \times 24 \times 5 / 8$ & W36X170 \\
\hline $32 \sim 33 / 33 \sim 34$ & W36X170 & W14X159 & $\mathrm{TS} 24 \times 24 \times 5 / 8$ & W36X170 \\
\hline $34 \sim 35 / 35 \sim 36$ & W33X118 & W14X90 & TS24x24x5/8 & W33X118 \\
\hline $36 \sim 37 / 37 \sim 38$ & W30X108 & W14X90 & TS $24 \times 24 \times 5 / 8$ & W30X108 \\
\hline $38 \sim 39 / 39 \sim 40$ & W24X94 & W14X90 & TS16x 16x1/2 & W24X94 \\
\hline 40 / Roof & W24X94 & W14X90 & TS16x 16x1/2 & W24X94 \\
\hline
\end{tabular}

Figure 2-3. Floor plan, elevations and beam and column sections of the 40-story building (Han et al., 2015) 


\subsection{Nonlinear Structural Analysis Models}

\subsubsection{Background}

To consider the nonlinearity for a structural component, different models with different levels of complexity of considering plasticity distribution, both over the cross section and along the length of the component, have been developed and utilized. Figure 2-4 illustrates five alternative idealized models considering inelasticity for beam-column elements in different degrees of idealization (more comprehensive model by moving from (a) to (e)). Plastic hinge and nonlinear spring hinge models, models (a) and (b) in Figure 2-4, constitute the simplest models by considering concentrated moment-rotation plasticity in a zero-length portion in two ends of the element and an elastic beam-column element between them. While the plastic hinge model deliberates rigid-plastic relationship as the moment-rotation plasticity, the nonlinear spring hinge model enables to consider elastic-plastic relationship including hysteretic properties.

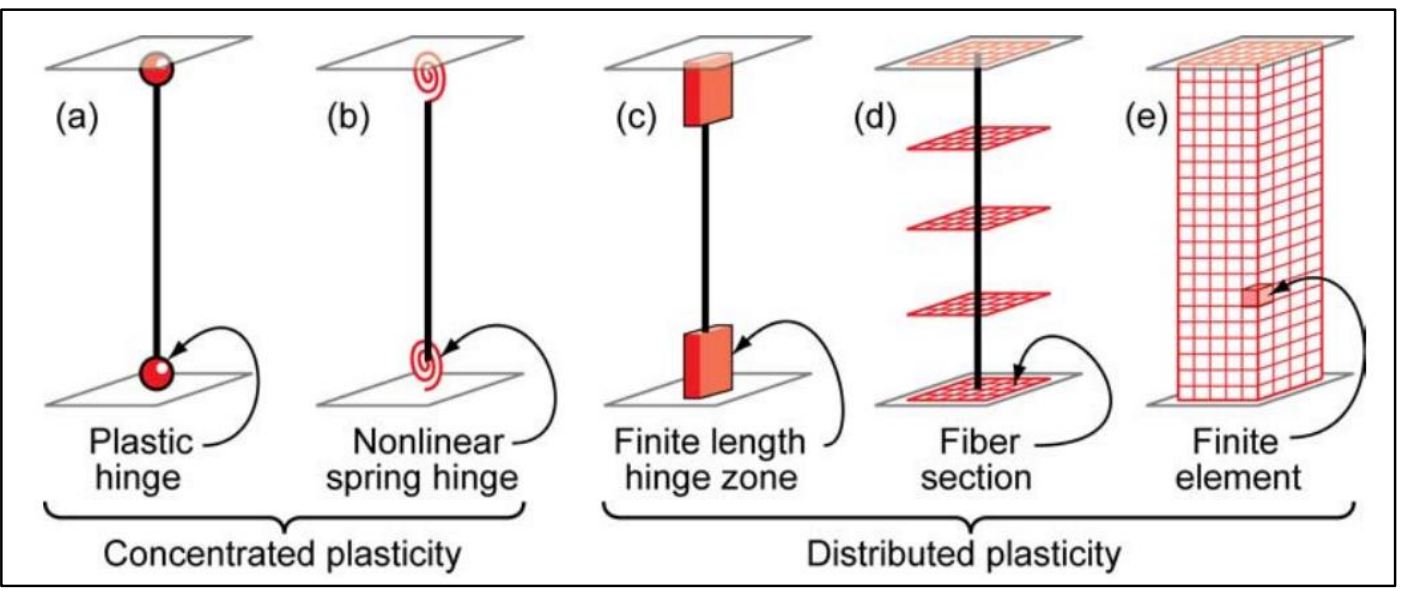

Figure 2-4. Idealized models representing inelasticity for beam-column elements (Deierlein, Reinhorn, \& Willford, 2010)

Models (c), (d) and (e) represent distributed plasticity models being utilized for considering inelasticity in beam-column elements. Model (c), finite length hinge zone, efficiently distributes the plastic deformation in finite lengths at two ends of the member which is more realistic compared to plasticity concentrated models. The nonlinear behavior in two ends can alternatively 
be provided by introducing a moment-rotation relationship or using Fiber Section Integration. The Fiber Section model, model (d), distributes the plasticity over the member cross sections and along the member length by numerical integrations. In this method, the cross section is discretized to small fibers which follow given uniaxial material models with nonlinear hysteretic axial stress-strain characteristics. At a few points along the member, called "integration points", the resultant forces, axial force and moments, are obtained by numerical integration of uniaxial stresses of fibers over the cross sections. This formulation requires the assumption that plane sections remain plane. Then, displacement or force interpolations are implemented to integrate the cross section responses at those discrete sections at integration points along the member length to calculate the overall force/deformation of the element. Finally, the finite mesh element model, model (e), is the most comprehensive and complex model where the whole member is discretized to small finite element along the member length and over the cross sections. The finite mesh elements follow given nonlinear hysteretic constitutive properties. Although this model avoids lots of simplifying assumption and numerical formulations such as integrations and interpolations as taken in other methods, this model is time-consuming and involves challenges regarding model parameter calibration and computational resources. Convergence of a numerical solution of the finite mesh element model in a huge model of a high-rise building, specifically during a transient analysis, is a major challenge pushing to use simplified approaches.

Compared to simplified models; zero-length and finite-length plastic hinge and nonlinear spring hinges, fiber-section, and finite mesh element models are capable of capturing axial load-bending moment interaction directly. However, they may not capture the degradations associated with local instabilities such as local buckling. Simpler models may be able to capture them by appropriate force-deformation relationships including the effect of the phenomena. 


\subsubsection{Characteristics of Cyclic Responses}

\section{Strength and Stiffness Degradation}

Structural components and systems subjected to cyclic loading exhibit some levels of decrease in lateral stiffness and strength which is called "strength and stiffness degradation". The deterioration represents the consequences of damage in the cyclic response of structural components. In structural steel structures, the phenomena in beams and columns that may contribute to deterioration are: (a) Local buckling of flanges or web; (b) Lateral-torsional buckling; and (c) Ductile tearing. Additional phenomena in connections which may cause deterioration include: (a) Crack propagation and fracture; (b) Bolt slippage, yielding, and bearing; (c) Block shear; (d) Prying action; (e) Local plate bending; (f) Plate compression buckling; and (g) Shear buckling of plates (PEER/ATC, 2010).

The consequences of deterioration in structural component's responses are early instability in lateral load-deformation responses and the sensitivity of peak strength to loading history (smaller peak strength than for monotonic response as a function of loading history). This fact raises questions about the reliability of the approach using monotonic response characteristics to assess deformation capacity either for structural components or for entire structure response. The characteristics of deterioration in cyclic response of components can be categorized into several modes of deterioration as follows (Ibarra \& Krawinkler, 2005; PEER/ATC, 2010):

- Mode 1 - Basic strength deterioration: This mode of deterioration is associated with number and amplitude of loading cycles even before reaching to cap strength. As shown in Figure 2-5, this deterioration is characterized by a translation of pre-capping strength bound toward the origin.

- Mode 2 - Post-capping strength deterioration: A cyclic strength deterioration beyond cap strength associated with negative tangent stiffness. This mode of deterioration is 
characterized by a translation of the post-capping strength bound towards the origin as shown in Figure 2-5.

- Mode 3 - Unloading stiffness deterioration: Cyclic unloading stiffness deterioration associated with number and amplitude of loading cycles. As shown in Figure 2-5, this mode of deterioration characterized by a rotation of the unloading slope.

- Mode 4 - Accelerated reloading stiffness deterioration: This cyclic stiffness deterioration is associated with the increase in strength in a cycle with larger deformation compared to a cycle with the same deformation. This cyclic deterioration can be observed in instances such as reinforced concrete beams subjected to a high shear force. However, it is not noticeable in components with behavior that is controlled by flexure. This mode of deterioration is characterized by movement of the point at which the strength envelope is reached away from the origin.

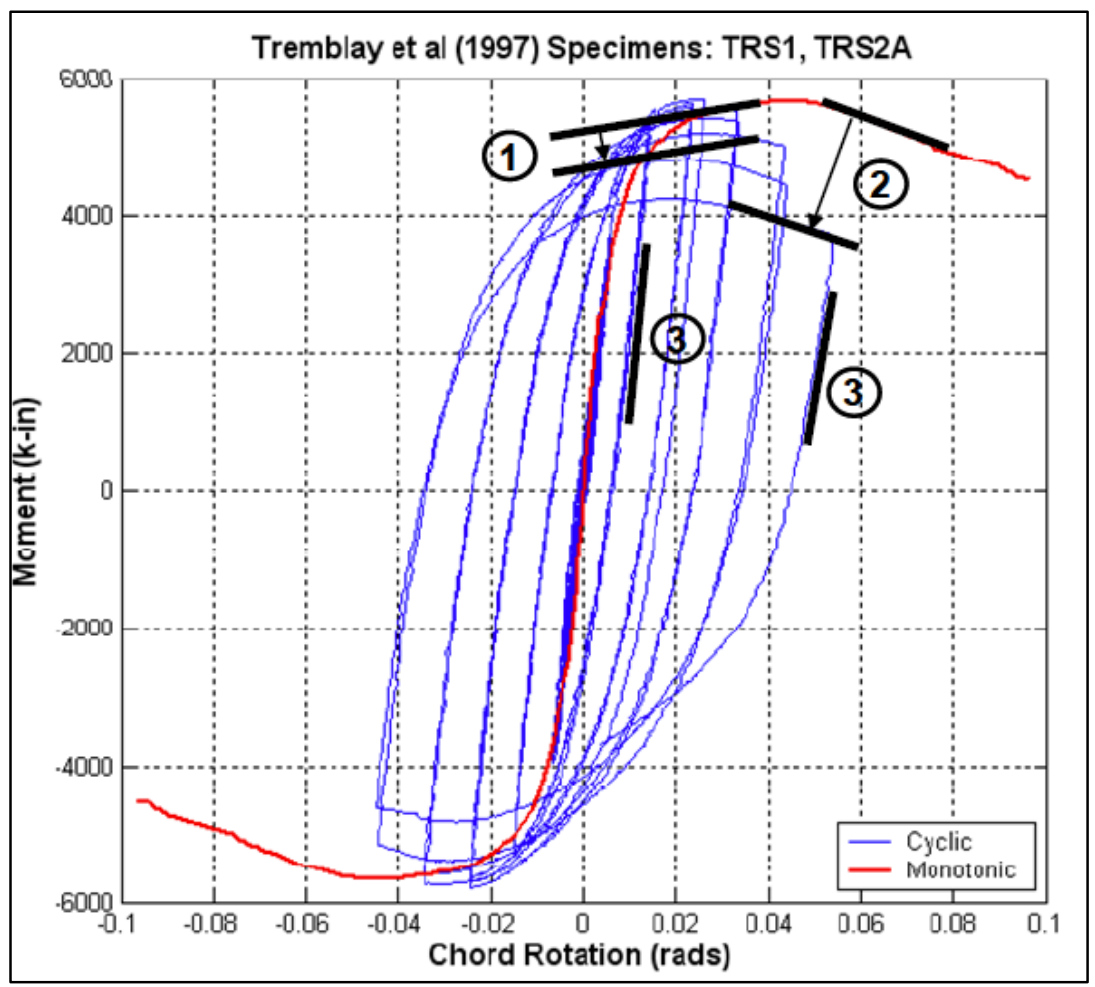

Figure 2-5. Modes of deterioration (PEER/ATC, 2010) 
As illustrated in Figure 2-6, two type of degradations are identified in the cyclic response of SDOF oscillators: Cyclic Degradation and In-cycle Degradation (FEMA, 2005). Cyclic Degradation is termed as the loss in stiffness and strength occurring in subsequent cycles (see Figure 2-6a), while the In-cycle Degradation is termed as the loss in strength and the loss in stiffness (negative stiffness) occurring within a cycle. It may occur as a result of geometric nonlinearities such as P-delta effect, as well as, material nonlinearities. Systems with cyclic degradation typically respond stable to cyclic excitation, but a system with in-cyclic degradation characteristics is prone to dynamic instability and collapse. Studies have shown that for systems with moderate and long natural period (systems with periods of vibration greater than 1.0s), assuming cyclic strength and stiffness degradation model result in the same peak displacement as obtained using elastic-plastic or bilinear strength hardening systems (FEMA, 2009).

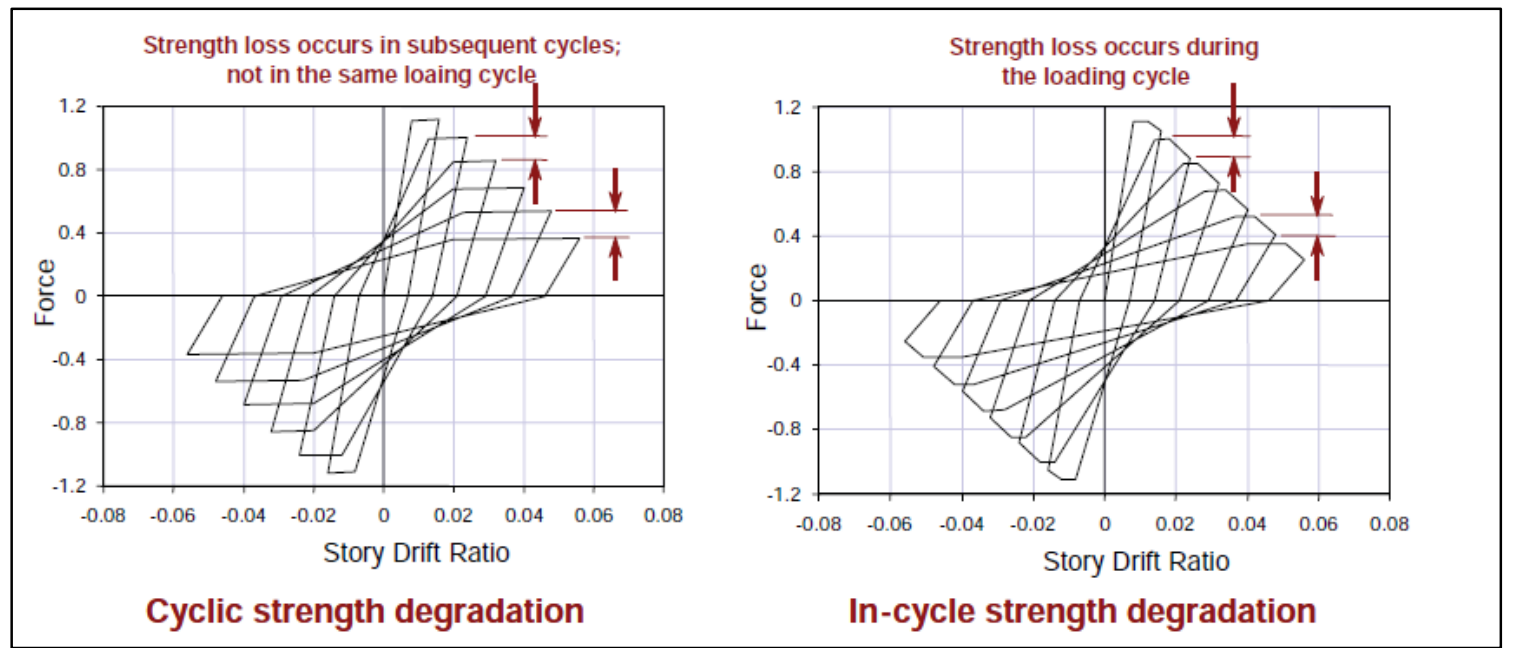

Figure 2-6. Two type of degradations in hysteresis response (FEMA, 2005; FEMA, 2009)

\section{Force-Displacement Backbone}

Generally, the overall limitation on the force-deformation behavior of structural components is shown in terms of backbone curves. In nonlinear analysis, backbone relationships are normally used to define component behavior along with performance evaluation parameters: demand and acceptance criteria parameters. The backbone curve concept may be alternatively used to: 
(1) represent the force-deformation behavior of structural components in nonlinear static pushover analysis of a structural system; or (2) envelop the force-displacement response of structural components undergoing cyclic or monotonic testing. It should be noticed that these backbone curves cannot be used interchangeably in component modeling. Although monotonic force-deformation data are useful for some impulse loading analysis, it generally corresponds with significantly less strength and stiffness degradation and consequently much greater strength and deformation capacity and unrealistic responses for longer dynamic actions. This is because the monotonic response does not characterize degradation phenomena such as large tensile cracks in concrete or fracture and buckling in steel, typically developed in nonlinear cyclic responses. Figure 2-7a shows the monotonic force-displacement backbone, representing the response that would be observed for a component tested under monotonic loading versus cyclic forcedisplacement backbone which is an envelope enclosing the force-displacement response to a specific cyclic loading.

In a nonlinear system under cyclic loading, the characteristics of cyclic force-displacement response envelope depend on the loading history. Takemura and Kawashima (1997) tested six identical reinforced concrete bridge piers with six different loading protocols. The obtained result showed significant different hysteretic responses, such that the loading protocols with increasing amplitudes and more cycles led to lower force-displacement response and protocols with decreasing amplitudes and fewer cycles resulted in larger force-displacement response envelopes. So it was concluded that the cyclic force-displacement envelope is influenced by the cyclic loading characteristics including the amplitude of each cycle, and the number and sequence of the loading cycles.

Accounting for the dependence of cyclic force-displacement envelope on loading characteristics, FEMA P-440-A (2009) introduces two terms of Cyclic Envelope and Capacity Boundary 
backbones as shown in Figure 2-7a. Cyclic Envelope backbone refers to the force-deformation response envelope for a certain cyclic loading. Typically, the Cyclic Envelope can be characterized by moving and rotation of the branches of the monotonic backbone curve toward the origin (Figure 2-7a). The Capacity Boundary backbone defines the maximum strength that a structural member can develop at a given level of deformation. The Capacity Boundary backbones are close but not necessarily identical with monotonic response curve and it may include some cyclic response characteristic such as the average effect of cyclic hardening in order to simplify response description (PEER/ATC, 2010).

The Capacity Boundary backbone can be obtained through one of following approaches: (1) refined analytical modeling; (2) a monotonic test; or (3) back-figuring from a cyclic test. When the backbone curve is obtained according to a standard cyclic loading protocol, the comparableness of the loading protocol and the existing loading history has a significant role on realistic modeling of the nonlinear hysteretic response of the component. For a loading with load/deformation history less than or equal to standard cyclic loading following Capacity Boundary backbone results in correct modeling as shown in Figure 2-7b. However, for a loading history with load/deformation greater than standard cyclic loading, following the Capacity Boundary backbone will result in unrealistic in-cycle degradations which significantly affect the response. Also, using intense cyclic standard loading protocols will result in underestimating the actual force-displacement capacity and consequently may lead to conservative Capacity Boundary backbones. Thus, standard cyclic loading protocols used for obtaining the Capacity Boundary backbone need to be close to the loading history that the component is expected to experience in the corresponding dynamic excitation. 

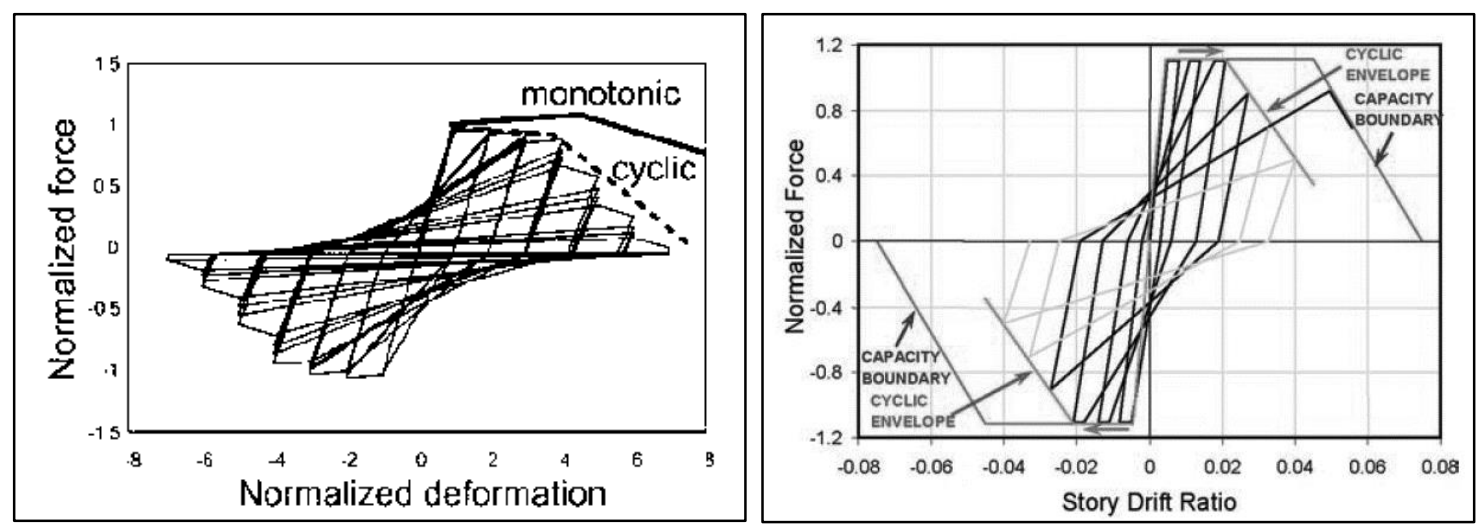

Figure 2-7. Force-displacement backbones; (a) cyclic backbone versus monotonic backbone (Deierlein et al., 2010), (b) cyclic envelope versus capacity boundary (FEMA, 2009)

In nonlinear dynamic analyses, the cyclic degradation in component response may be modeled directly or be considered indirectly. By directly modeling cyclic degradation, the response follows the monotonic backbone at the beginning and then degrades according to the characteristics of the cyclic loading. In indirect modeling of cyclic degradation an appropriate cyclic backbone with the implied amount of the degradation needs to be specified.

\subsubsection{Nonlinear Hysteretic Models of Structural Components}

In past couple of decades, several models have been proposed to represent nonlinear hysteresis response of structural components (Chenouda \& Ayoub, 2008; Ibarra, Medina, \& Krawinkler, 2005; Kunnath, Mander, \& Fang, 1997; Mostaghel, 1999; Sivaselvan \& Reinhorn, 2000). While these models utilize different approaches to incorporate the degradations, they all share three common concepts to represent the degradation model: (a) defined force-deformation backbone curves distinguishing the strength capacity that a structural member can develop at a given deformation; (b) a set of rules defining the basic characteristics of hysteric behavior inside the backbone bounds; and (c) a set of rules incorporating different types of deterioration in the model (PEER/ATC, 2010).

For a structural component, the backbone load-deformation relationship defines the bounds confining the hysteretic response of the component. The hysteresis response of the component 
cannot cross the backbone and for a certain deformation, when the force reaches the maximum capacity defined by the backbone curve, the response will continue along the curve. This behavior corresponds to the in-cycle strength degradation in that specific cycle. For a response with no cyclic degradation the response follows the backbone curve. Figure 2-8 shows the general idealized force-deformation backbone for nonlinear dynamic models of components as suggested by ASCE/SEI 41-06 (ASCE, 2007). As shown in the figure the backbone load-deformation response initiates with an elastic response, followed by a hardening response which ends up to peak strength point. The peak strength displacement corresponds to the onset of the strength degradation of the component. The strength degrades within a negative slope portion to reach the constant or slightly decreasing residual strength portion which concludes to the final failure of the component.

A full backbone Force-deformation relationship as shown Figure 2-8 in is characterized by specifying some key parameters which can be categorized into: stiffness parameters; strength parameters; and deformation parameters. The stiffness parameters include: elastic stiffness, strain hardening stiffness, post-capping stiffness. The strength parameters include: yield strength, peak (capping) strength and residual strength. The deformation parameters are defined in accordance with strength parameters. Therefore, the deformation parameters include: Yielding deformation, peak strength deformation, deformation associated with the onset of residual response, and ultimate deformation. 


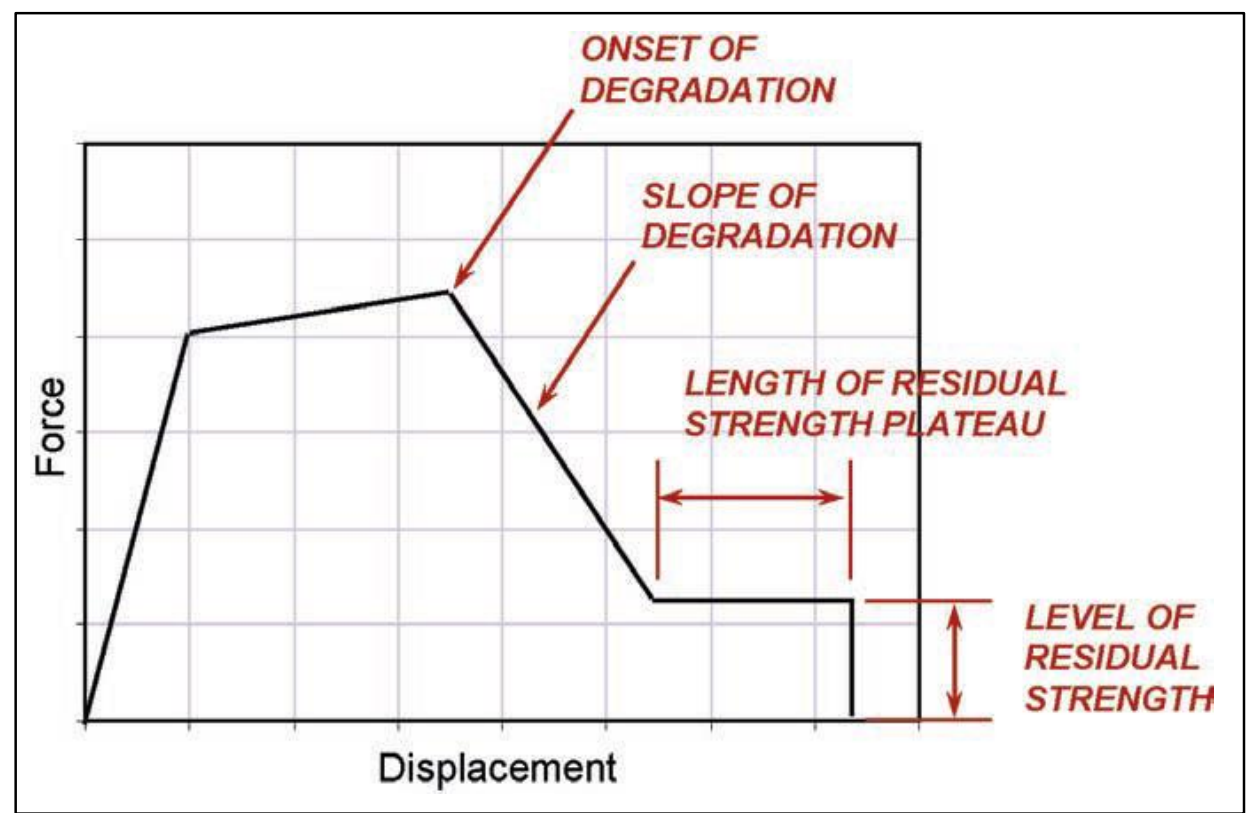

Figure 2-8. Typical force-deformation backbone for nonlinear dynamic models of components (FEMA, 2009)

For a structural component with a given backbone curve, basic hysteretic rules need to be established to represent the characteristics of the overall shape of the hysteretic response. The rules may follow either basic linearized models such as bilinear, peak oriented and pinching response models shown in Figure 2-9, or more refined hysteresis models such as multilinear models. Finally, cyclic deterioration rules need to be considered to represent the cyclic deterioration characteristics depending on the loading history. The cyclic deterioration in each cycle is typically defined in accordance with the energy dissipated in the cycle with respect to an inherent reference hysteretic energy dissipation capacity which is independent of the loading history applied to the component. 


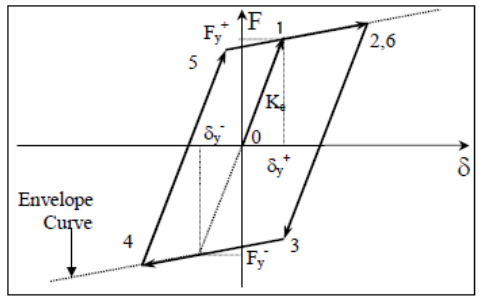

(a)

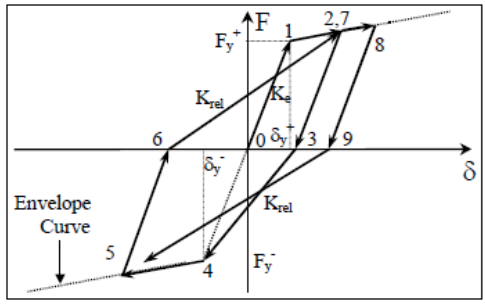

(b)

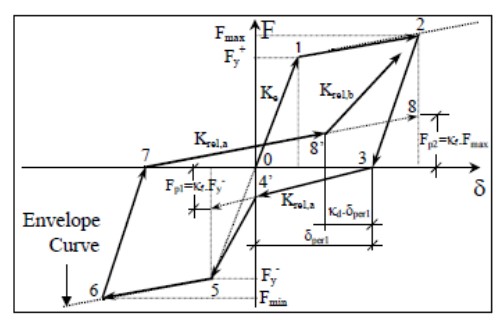

(c)

Figure 2-9. Basic linearized hysteresis models: (a) bilinear; (b) peak oriented; and (c) pinching (PEER/ATC, 2010)

\subsection{Modeling Techniques and Procedures}

\subsubsection{Background}

Generally, the nonlinear modeling of structural components includes defining nonlinear behavior characteristics in addition to the basic physical characteristics used to establish linear components. For instance, a linear beam or column component modeled as a line element can be simply defined by distinguishing the material properties such as modulus of elasticity and geometry of cross-section such as area and moment of inertia. However, modeling nonlinear beam or column components requires establishing nonlinear behavior characteristics either implicitly in a finite element model by specifying comprehensive material constitutive models or explicitly in a concentrated plasticity model by defining full force-deformation relationships (shown in Figure 2-8) and cyclic degradation rules.

Creating comprehensive reliable nonlinear analytical models of the three described steel moment frame building involves primary evaluations and engineering judgments of the expected inelasticity developments and failure modes due to extreme wind dynamic lateral loading. As a steel moment resisting system, the developed model included flexural nonlinear component models for beams and columns. The ordinary or intermediate moment frame design procedures of the buildings do not guarantee the inelasticity to be only developed in flexural hinges in the beams and the column bases as would be obtained by using Special Moment Frame Design 
principles. Therefore, in addition to flexural members, the inelastic models for panel zones and connections were also considered as well as the inquiries on inelasticity and failure of other load actions such as member shear yielding and member instabilities due to local or lateral-torsional buckling.

In this study, beam-column elements are modeled using concentrated plasticity models by using zero-length nonlinear spring models. This was in spite of using fiber-section model in earlier studies. Although fiber-section models enable more accurate modeling of initiation of inelasticity effects, particularly initiation and spread of yielding in steel structures, it has major issues in capturing the cyclic degradation as well as the degradations associated with local buckling and fracture of members in steel structures (Deierlein et al., 2010; FEMA, 2009). This deficiency significantly affects its reliability to predict a realistic response in post-peak and collapse prediction analyses. Therefore, a concentrated zero-length nonlinear spring model utilizing a calibrated moment-rotation relationship is more practical to capture the whole nonlinear response specifically in post-peak and collapse prediction analyses. For this reason, a zero-length nonlinear spring model was used in the final analyses.

Unlike concrete moment frames where the nonlinear response of beams and columns are coupled with behavior of panel zone due to possible bond slip of longitudinal reinforcements, in steel moment frames the inelastic components, beam, column, panel zone and connections, most likely deform independently to the extent that one component reaches maximum capacity and the system fails. Therefore, a reliable panel zone model was adopted and used in this analytical approach to explicitly incorporate nonlinear response of panel zones.

\subsubsection{Types of Force Action in Structural Components}

The response of a structural component to lateral load may result in the development of different types of force actions such as: axial load $(\mathrm{P})$, shear $(\mathrm{V})$, bending moment $(\mathrm{M})$ and torsion $(\mathrm{T})$, or 
any combination of them. To evaluate the overall performance of a component, all force actions and the corresponding types of component response, either displacement-controlled (ductile) or force-controlled (brittle), need to be recognized. Table 2-1 shows examples of force actions developed in moment and braced frames and their possible response type (displacementcontrolled or force-controlled). It is shown that a given component may include both displacement-controlled and force-controlled actions.

Table 2-1. Force actions and their possible response type (ASCE, 2014)

\begin{tabular}{|c|c|c|}
\hline Component & $\begin{array}{l}\text { Deformation-Controlled } \\
\text { Action }\end{array}$ & Force-Controlled Action \\
\hline $\begin{array}{l}\text { Moment frames } \\
\text { - Beams } \\
\text { - Columns } \\
\text { - Joints }\end{array}$ & $\begin{array}{l}\text { Moment }(M) \\
-\end{array}$ & $\begin{array}{l}\text { Shear }(V) \\
\text { Axial load }(P), V \\
V^{a}\end{array}$ \\
\hline Shear walls & $M, V$ & $P$ \\
\hline $\begin{array}{l}\text { Braced frames } \\
\text { - Braces } \\
\text { - Beams } \\
\text { - Columns } \\
\text { - Shear link } \\
\text { Connections } \\
\text { Diaphragms }\end{array}$ & $\begin{array}{l}P \\
\overline{-} \\
V \\
P, V, M^{b} \\
M, V^{c}\end{array}$ & $\begin{array}{l}\bar{P} \\
P \\
P, M \\
P, V, M \\
P, V, M\end{array}$ \\
\hline
\end{tabular}

According to ASCE 7-10-13, Type 1 and Type 2 backbone curves shown in Figure 2-10 present the typical force-deformation backbone curve for deformation-controlled (ductile) actions. But it also has a requirement depending on their component type (classified as primary and secondary). For force-displacement curve type 1 , primary components are required to meet $d \geq 2 g$, while all secondary type components are considered displacement-controlled. For force displacement curve type 2 , primary components are required to meet $e \geq 2 g$, and secondary type components are required to meet $f \geq 2 g$ to be considered displacement-controlled. Figure 2-10 shows d, e and g parameters. A typical force-controlled response curve is shown in Figure 2-10 as a Type 3 curve. All primary response backbone curves with this type of force action force-displacement response 
are considered force-controlled, however, secondary components with this type of response still can be considered displacement-controlled if they meet the $f \geq 2 g$ requirement.

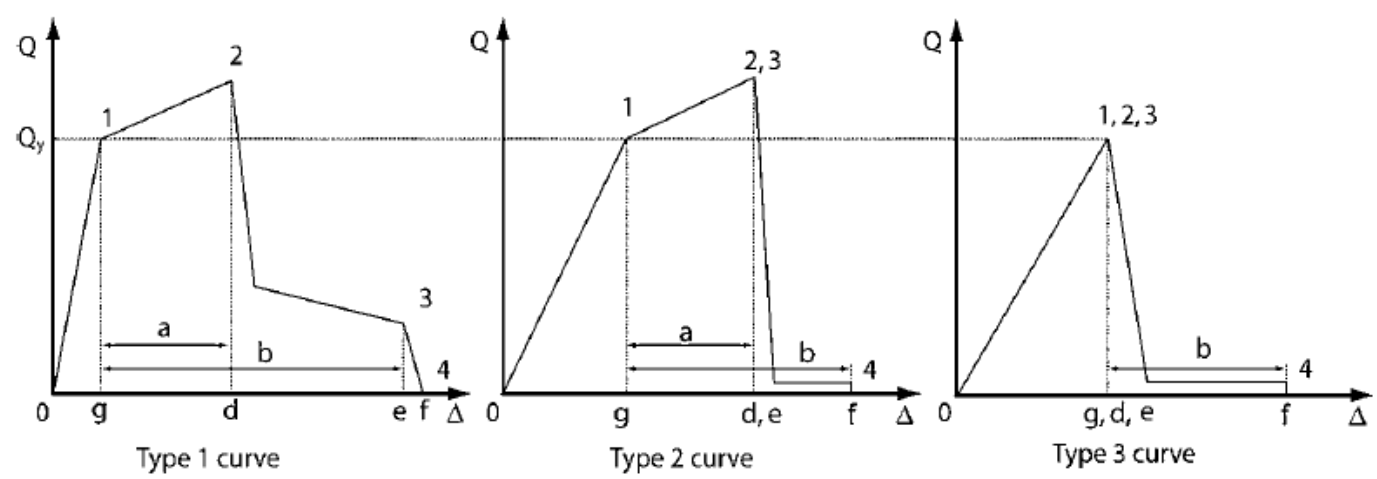

Figure 2-10. Force-deformation backbones for different types of force actions in structural components

The generalized force-deformation backbone curve suggested by ASCE 7-10-13 for deformation-controlled actions is shown in Figure 2-11a. The response backbone curve initiates with an elastic response from point A (the fully unloaded condition) to point B (the effective yield point). It then continues with a slight slope representing the strain hardening to the peak strength point C. Then the component experiences a significant strength degradation. While the strength degradation (C to D) may happen as a sudden drop, it may be provided with a slope to avoid computational difficulty and inability to converge associated with a sharp transition. Point D to point E represents the residual strength which may be considered as a constant or degrading amount. Point E represents the displacement where the component fails to carry the load anymore. It may result in overall instability where the structure does not have sufficient redundancy to redistribute the forces to other structural elements. Figure 2-11a illustrates typical acceptance criteria for deformations corresponding to the target Building Performance Levels of Collapse Prevention (CP), Life Safety (LS), and Immediate Occupancy (IO). 


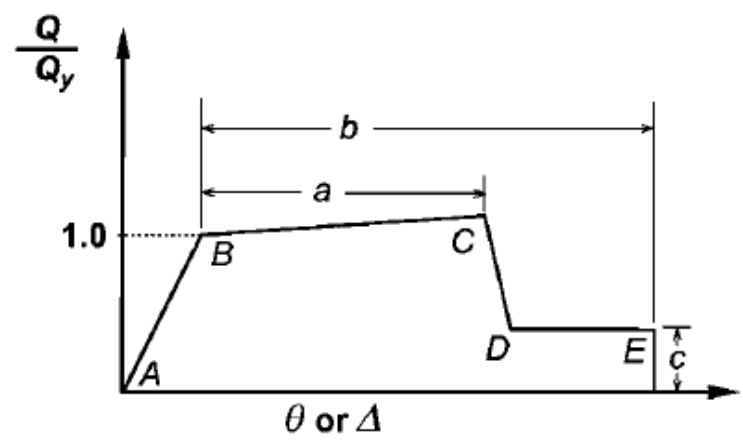

(a)

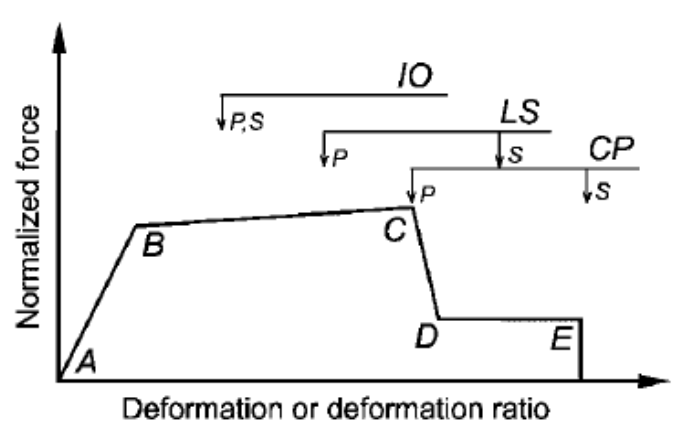

(b)

Figure 2-11. ASCE41-13 component response of deformation-controlled actions: (a) forcedeformation relationship, (b) acceptance criteria

\subsubsection{Component Capacity}

In evaluating the behavior of deformation-controlled actions, ASCE 7-10-13 recommends using Expected Strength $Q_{C E}$ for yield strength of the component $Q_{y}$ in the force-deformation response backbone. $Q_{C E}$ is determined as the mean value of resistance of a population of similar components. In evaluating the behavior of force-controlled actions $Q_{C L}$, Lower-Bound strength is recommended to be used for the yield strength of the component $Q_{y} . Q_{C L}$ is determined as the mean value minus a standard deviation of resistance of a population of similar components. Where the component capacities are calculated based on the material properties, as for the 47story building, the Nominal material properties, or properties specified in construction documents are considered as the lower-bound material properties. The Expected material properties can be determined by multiplying the lower-bound material properties by a specified factor.

\section{Material Properties}

The steel used for structural components in the three high-rise buildings is ASTM A-572 Grade 50 which has been considered as the lower-bound for material properties in accordance with ASCE 7-10-13 standard. ASCE 41-13 standard specifies different lower-bound tensile and yield strengths for A-572 Grade 50 based on their shape size group, but in this study the minimum 
amounts, lower-bound Yielding Strength= $50 \mathrm{kips} / \mathrm{in}^{2}$ and lower-bound Tensile Strength= 65 kips/in ${ }^{2,}$ were used for all steel structural components. The expected material properties were also determined by multiplying lower-bound material properties by the factor given by ASCE 7-10-13 standard. According to the standard, the factor 1.1 was selected to calculate the expected material properties based on known lower-bound material properties. Therefore, the expected Yielding Strength $55 \mathrm{kips} / \mathrm{in}^{2}$ and expected Tensile Strength $71.5 \mathrm{kips} / \mathrm{in}^{2}$ were used for all steel structural components. Lower-bound material properties and expected material properties have been used in nonlinear dynamic evaluation for force-controlled actions and deformation-controlled actions respectively.

\subsubsection{Selecting The Nonlinear Modeling Approach}

ASCE 7-10-13 “seismic evaluation and retrofit of existing buildings," provided a framework for nonlinear modeling of structural components. As described earlier, the recommended modeling approach for displacement-base (ductile) members is characterized by force-deformation relationships and corresponding modeling parameters such as a, $b$ and $c$ shown in Figure 2-11a. The main shortcoming associated with using this model in the nonlinear dynamic analysis is that this model is intended to be used for static nonlinear analysis (pushover). The modeling parameters a, $b$ and $c$ define a backbone strength which already includes the cyclic deterioration expected in seismic evaluation and the model does not include any rules for cyclic deterioration. As already discussed the cyclic deterioration of structural component is very sensitive to the loading scenario. So the model may not be reliable for using in wind dynamic analysis with significantly different dynamic loading nature.

The ASCE 7-10-13 model does not provide information regarding the post-capping (C to $\mathrm{D}$ ) stiffness where rapid strength drop is shown in the recommended model. This steep degradation is not a valid assumption at least for steel beams. Also considering a sudden strength drop as 
shown in the model will lead to issues of numerical stability and convergence even in static pushover analysis. ASCE 7-10-13 provides modeling parameters in terms of yielding rotation of multiples of $\theta_{y}$ which itself is sensitive to span to depth ratio.

An alternative approach available for modeling nonlinearity of structural components is the approach recommended in PEER/ATC-72 incorporating a Modified Ibarra-Krawinkler deterioration model. The model incorporates monotonic backbone and a set of rules defining the cyclic deteriorations depending on the loading characteristics. The modeling parameters associated with this model were developed through extensive efforts by calibrating the model with a large number of experimental data. More detailed explanations related to this approach is provided in the following section.

Given the facts noted above, the approach recommended in PEER/ATC-72 was found more appropriate for nonlinear wind loading analysis in this study. The following section describes the development of structural components mainly according to the PEER/ATC-72 approach.

\subsubsection{Nonlinear Hysteretic Modeling of Steel Beams and Columns}

In this study, the steel beams and columns were modeled as lumped plasticity elements. In this approach, each beam/column component was modeled by an elastic element with concentrated plastic hinges the ends. The plastic hinges are to be located where it well presents the integrated effect of distributed inelasticity. Therefore, the column plastic hinges were located at the column ends, right below and above the panel zone. Beam plastic hinges were located at the ends rather distant from column face. The lumped plasticity hinges were represented by nonlinear rotational springs following cyclic force-deformation relationships defined by a backbone curve and deterioration rules.

Accurate modeling of deterioration characteristics of structural components is required to reliably assess the extensively nonlinear and near collapse performances of the structures when the 
building is exposed to high-intensity dynamic excitations. Even the use of refined finite element models which dramatically increase the running time of the analysis and make it more difficult to converge, does not guarantee the accurate simulation of degradation. The model must include considerations for all important sources of deterioration, and incorporate cyclic degradation phenomena. Cross-section based models such as the fiber section model, have a major deficiency in accounting for the sources of deterioration that cannot be included by cross-sectional properties such as shear nonlinearity and post-buckling behavior in steel members.

In recent years, several studies focused on developing reliable deterioration models for structural components in order to advance structural collapse prediction analysis (Ibarra, Medina, \& Krawinkler, 2002; Ibarra \& Krawinkler, 2005; Zareian, Lignos, \& Krawinkler, 2010). Some experimental result databases (PEER database, NEES database and SAC database) have also provided monotonic and cyclic loading test results of versatile structural components. The experimental results have been used to validate the suggested models and assess their reliability as a deterioration model of a structural component. The studies have made significant progress in modeling of the deterioration of structural components. However, reliably predicting deterioration properties of the structural component and properly incorporating them in numerical analysis still is a major challenge in near-collapse and collapse prediction analyses.

According to PEER/ATC-72 (2010), the deterioration of a structural component can be incorporated into the analytical model through following three approaches: (1) the cyclic deteriorations can be explicitly incorporated into the model by defining a backbone curve which moves toward the origin with a rate depending on the loading history; (2) using a given cyclic backbone as a modified backbone curve, loading history related to the cyclic backbone requires being comparable with expected loading history; (3) factors can be used to modify the shape of backbone curve to account for cyclic deterioration. These factors depend on the material 
properties and component configurations and can be determined based on the experimental data. In cases where the post-capping response of the structure is not of interest, no deterioration model can be used. In this case, ultimate deformation of a component should be limited to the deformation associated with $80 \%$ of the capping strength.

In this study, the nonlinearity of beams and columns in moment-resisting frames was modeled based on the Modified Ibarra- Krawinkler (MIK) model (Lignos \& Krawinkler, 2010a). The model specifies the moment-rotation boundary as a monotonic backbone curve shown in Figure 2-12a. the multilinear backbone curve is defined by a set of strength (moment) and deformation (rotation) parameters. The strength parameters include; effective yield moment $M_{y}$, capping moment strength $M_{C}$ and residual moment $M_{r}$. The two latter parameters are defined as a ratio of effective yield moment such that, capping moment $M_{C}$ is defined by the post-yield strength ratio $M_{C} / M_{y}$ and residual moment $M_{r}$ is defined as $M_{r}=\kappa . M_{y}$, where $\kappa$ is the residual moment ratio. The deformation (rotation) parameters include yield rotation $\theta_{y}$, pre-capping plastic rotation $\theta_{P}$, post-capping plastic rotation $\theta_{P c}$ and ultimate rotation capacity $\theta_{u}$.

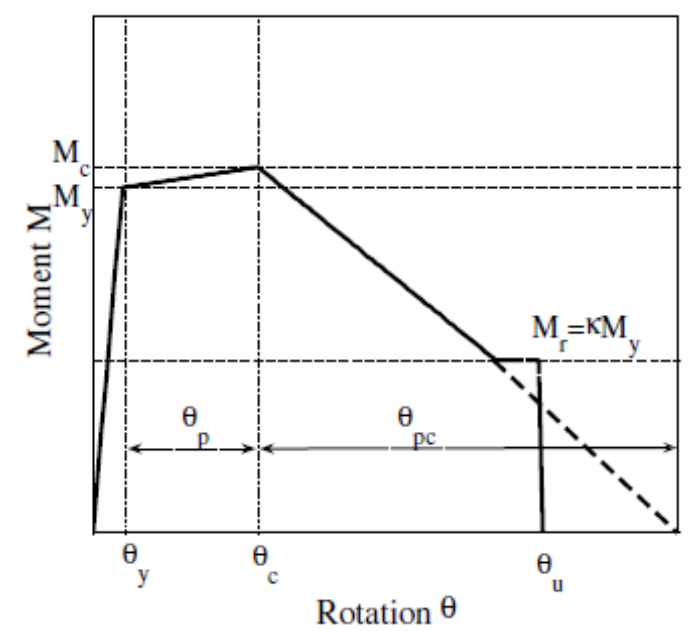

(a)

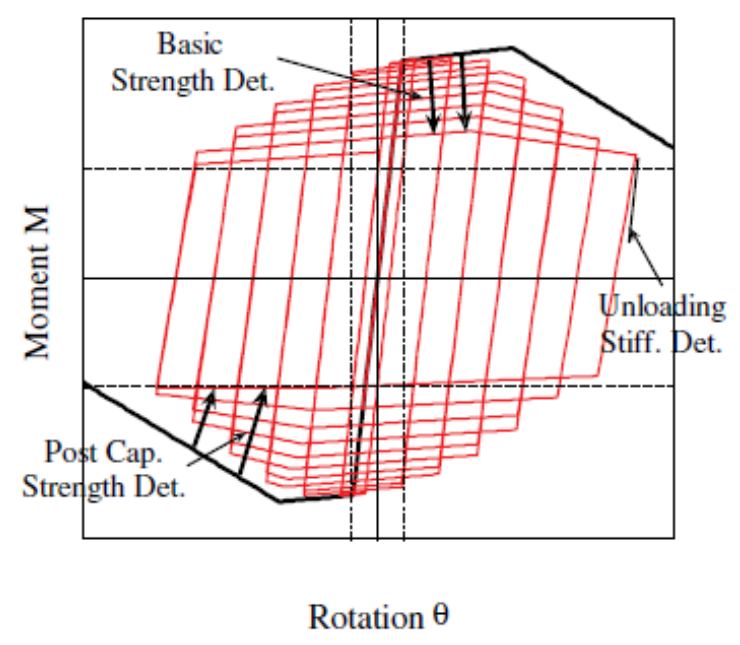

(b)

Figure 2-12. Modified IK deterioration model: (a) backbone curve; (b) cyclic response and deterioration characteristics (Lignos \& Krawinkler, 2010a) 
The deterioration model involves three modes of cyclic deterioration defining the characteristics of hysteretic behavior with respect to the backbone curve. As shown in Figure 2-12b, basic strength deterioration, post-capping strength deterioration, and unloading/reloading stiffness deterioration are three modes of deterioration incorporated in this deterioration model. The rate of cyclic deterioration is controlled by reference energy dissipation capacity $E_{t}$ which is an inherent property of each structural component regardless of the loading history. The reference energy dissipation capacity is expressed in terms of yield moment and the reference cumulative rotation capacity $\Lambda$ :

$$
E_{t}=\Lambda \cdot M_{y}
$$

The cyclic strength and stiffness deterioration are applied by translating the backbone strength toward the origin and gradually decreasing the loading and unloading slope respectively. The rate of the strength and stiffness deterioration are controlled by following relationships:

$$
\begin{gathered}
M_{i}=\left(1-\beta_{i}\right) \cdot M_{i-1} \\
K_{i}=\left(1-\beta_{i}\right) \cdot K_{i-1} \\
\beta_{i}=\left(\frac{E_{i}}{E_{t}-\sum_{j=1}^{i-1} E_{i}}\right)^{c}
\end{gathered}
$$

Where $M_{i}, K_{i}$ are reference strength bound and reference stiffness in each cycle respectively, $\beta_{i}$ is the energy-based deterioration parameter, $E_{i}$ is the hysteretic energy dissipated in each cycle and $\mathrm{c}$ is an empirical parameter, usually taken as 1.0. By choosing different $\Lambda$ values for each mode of deterioration different deterioration rates can be obtained.

Generally, the deterioration in the hysteretic response of steel beams and columns significantly depends on the development of local and lateral torsional buckling in plastic region. Steel buckling has been extensively studied but its effect on steel component behavior cannot be 
confidently represented in numerical models (even finite element models). Therefore, developing empirical rules based on the geometry parameters of the component has been the only practical solution to establish reliable hysteretic models. To this end, Lignos and Krawinkler (2010) provided databases for steel and concrete moment-resisting frame components providing calibrated MIK parameters based on geometric and material properties and detailing criteria. Among the three provided databases of concrete beams, steel wide flange beams, and steel tubular sections databases, the two latter databases were used in this study to incorporate the deterioration models for all structural components.

\section{MIK Parameters' Equations for steel W-sections}

Lignos and Krawinkler (2010) proposed a set of multivariate regression equations to correlate the Modified Ibarra-Krawinkler (MIK) model parameters to geometric and material parameters of steel $\mathrm{W}$-sections. To this end, the trend and dependence of MIK parameters to geometric and material parameters were first evaluated by investigating the parameters obtained through a calibration process in which the MIK model parameters were determined by matching the modeled moment-rotation relationship to experimental results of the $\mathrm{W}$-sections steel database. Through an observation of the trends following six parameters are found to be most influential: (1) depth of the beam $d$, (2) depth-to-thickness ratio of the Beam Web $\frac{h}{t_{w}}\left(h\right.$ and $t_{w}$ are the depth and the thickness of the beam web respectively), (3) width-to-thickness ratio of the beam flange $\frac{b_{f}}{2 . t_{f}}\left(b_{f}\right.$ and $t_{f}$ are the depth and the thickness of the beam flange respectively), (4) Shear Span-toDepth Ratio $\frac{L}{d}$ ( $L$ and $d$ are the span length and depth of the beam respectively), (5) sensitivity to lateral torsional buckling $\frac{L_{d}}{r_{y}}\left(L_{d}\right.$ and $r_{y}$ are the unbraced length and beam radius of gyration in weak axis respectively) and (6) expected yield strength of the flange of the beam $F_{y}$. 
The observed trend in the study showed that increase in beam depths usually results in a decrease in plastic rotational capacity. Respecting span to beam depth ratio, a linear proportional trend to rotational capacity was observed. However, the dependence was less significant in beam depth larger than $21 \mathrm{in.} \mathrm{As} \mathrm{expected,} \mathrm{large} \mathrm{flange} \mathrm{and} \mathrm{web} \mathrm{slenderness} \mathrm{ratios,} \mathrm{as} \mathrm{well} \mathrm{as} \mathrm{large} \mathrm{torsional}$ buckling parameters, have detrimental effect on all three modeling parameters including precapping plastic rotation $\theta_{P}$, post-capping plastic rotation $\theta_{P c}$ and reference cumulative rotation capacity $\Lambda$.

Nonlinear regression equations were then developed by Lignos and Krawinkler to determine MIK deterioration parameters including pre-capping plastic rotation $\theta_{P}$, post-capping plastic rotation $\theta_{P C}$ and reference cumulative rotation capacity $\Lambda$ for steel w-sections. The equations were developed considering two categories of "for the entire range of data" and "sections with $d \geq 21$ in". The following section shows the proposed equations for W-section beams, which are not Reduced Section Beams (RSB), used in this study.

- Pre-capping Plastic Rotation

○ Type1: for the entire range of data

$$
\theta_{p}=0.0865 \cdot\left(\frac{h}{t_{w}}\right)^{-0.365} \cdot\left(\frac{b_{f}}{2 . t_{f}}\right)^{-0.14} \cdot\left(\frac{L}{d}\right)^{0.34} \cdot\left(\frac{c_{u n i t}^{1} \cdot d}{533}\right)^{-0.721} \cdot\left(\frac{c_{u n i t}^{2} \cdot F_{y}}{355}\right)^{-0.230}
$$

○ Type2: For $d \geq 21$ in

$$
\theta_{p}=0.318 \cdot\left(\frac{h}{t_{w}}\right)^{-0.550} \cdot\left(\frac{b_{f}}{2 . t_{f}}\right)^{-0.345} \cdot\left(\frac{L_{d}}{r_{y}}\right)^{-0.023} \cdot\left(\frac{c_{u n i t}^{1} \cdot d}{533}\right)^{-0.33} \cdot\left(\frac{c_{u n i t}^{2} \cdot F_{y}}{355}\right)^{-0.130}
$$

- Post-capping Plastic Rotation

- Type1: for the entire range of data

$$
\theta_{p}=5.63 \cdot\left(\frac{h}{t_{w}}\right)^{-0.565} \cdot\left(\frac{b_{f}}{2 . t_{f}}\right)^{-0.8} \cdot\left(\frac{c_{u n i t}^{1} \cdot d}{533}\right)^{-0.28} \cdot\left(\frac{c_{u n i t}^{2} \cdot F_{y}}{355}\right)^{-0.430}
$$


○ Type2: For $d \geq 21$ in

$$
\theta_{p}=7.50 \cdot\left(\frac{h}{t_{w}}\right)^{-0.61} \cdot\left(\frac{b_{f}}{2 \cdot t_{f}}\right)^{-0.71} \cdot\left(\frac{L_{d}}{r_{y}}\right)^{-0.11} \cdot\left(\frac{c_{u n i t}^{1} \cdot d}{533}\right)^{-0.161} \cdot\left(\frac{c_{\text {unit }}^{2} \cdot F_{y}}{355}\right)^{-0.320}
$$

- Reference Cumulative Plastic Rotation

Type1: for the entire range of data

$$
\Lambda=495 \cdot\left(\frac{h}{t_{w}}\right)^{-1.34} \cdot\left(\frac{b_{f}}{2 . t_{f}}\right)^{-0.595} \cdot\left(\frac{c_{u n i t}^{2} \cdot F_{y}}{355}\right)^{-0.360}
$$

○ Type2: For $d \geq 21$ in

$$
\Lambda=536 \cdot\left(\frac{h}{t_{w}}\right)^{-1.26} \cdot\left(\frac{b_{f}}{2 . t_{f}}\right)^{-.0525} \cdot\left(\frac{L_{d}}{r_{y}}\right)^{-0.130} \cdot\left(\frac{c_{u n i t}^{2} \cdot F_{y}}{355}\right)^{-0.291}
$$

Note, $c_{u n i t}^{1}$ and $c_{u n i t}^{2}$ are constants related to unit conversions.

In this study, modeling parameters $\theta_{p}, \theta_{p c}$ and $\Lambda$ of all $\mathrm{W}$-section beams columns were acquired according to the above equations. For beams and columns with $d<21$ in and $d \geq 21$ in Type 1 and Type 2 equations were respectively used. The modeling parameters for $\mathrm{W}$-section columns were then modified based on their axial load as descried in a later section.

\section{MIK Parameters' Equations for Hollow Section Columns}

Lignos and Krawinkler conducted the same procedure performed for steel W-sections to develop regression equations to correlate the MIK model parameters with geometric and material parameters of steel hollow section columns. A database of more than 120 experimental data of HSS columns was collected to evaluate the deterioration properties of HSS columns under varying axial load and cyclic or monotonic bending. The investigation on trends of component deterioration parameters showed that the deterioration modeling parameters $\theta_{p}, \theta_{p c}$ and $\Lambda$ are

mostly depend on three parameters; (1) depth to thickness ratio $\frac{D}{t}$ ( $D$ and $t$ are column depth and 
thickness respectively), (2) axial load ratio $\frac{N}{N_{y}}$ ( $N$ and $N_{y}$ are the axial load and axial yield strength of the column respectively), and (3) expected yield strength of the flange of the beam $F_{y}$. Nonlinear regression equations were then developed considering three mentioned parameters. The following shows the proposed equations for HSS columns:

- Pre-capping Plastic Rotation

$$
\theta_{p}=0.614 \cdot\left(\frac{D}{t}\right)^{-1.05} \cdot\left(1-\frac{N}{N_{y}}\right)^{1.18} \cdot\left(\frac{C \cdot F_{y}}{380}\right)^{1.18}
$$

- Post-capping Plastic Rotation

$$
\theta_{p c}=13.82 \cdot\left(\frac{D}{t}\right)^{-1.22} \cdot\left(1-\frac{N}{N_{y}}\right)^{3.04} \cdot\left(\frac{c \cdot F_{y}}{380}\right)^{-0.15}
$$

- Reference Cumulative Plastic Rotation

$$
\Lambda=3012 \cdot\left(\frac{D}{t}\right)^{-2.49} \cdot\left(1-\frac{N}{N_{y}}\right)^{3.51} \cdot\left(\frac{C \cdot F_{y}}{380}\right)^{-0.2}
$$

Note, $c$ is a constant related to unit conversions.

In this study, modeling parameters $\theta_{p}, \theta_{p c}$ and $\Lambda$ of all box columns were acquired according to the above equations. For non-square box columns dimensions in larger direction were used to estimate conservative modeling parameters.

\section{Effective Yield Strength $M_{y}$ and Post-Yield Strength Ratio $M_{C} / M_{y}$}

In the modified Ibarra-Krawinkler model, isotropic hardening is accounted by increasing the yielding strength (moment) to an effective yield strength (moment). Typically, the effective yield moment is obtained by fitting best bilinear approximation to monotonic moment-rotation curve with an allowance for cyclic hardening which is typically equal to an amount slightly larger than 
the predicted bending moment strength $M_{p}$ (the plastic section modulus $\mathrm{Z}$ times the expected material yield strength). Statistical analysis conducted by Lignos and Krawinkler on the database resulted in mean and standard deviation of effective yield moment to expected moment strength ratio $M_{y} / M_{p}$ for beams (not RSB) which are equal to 1.17 and 0.21 respectively. The study also showed the mean and standard deviation of capping moment to effective yielding moment ratio $M_{c} / M_{y}$ for beams (not RSB) are equal to 1.11 and 0.05 respectively. In this study, effective yield moment to expected moment strength ratio $M_{y} / M_{p}$ and capping moment to effective yielding moment ratio $M_{c} / M_{y}$ equal to 1.17 and 1.10 were respectively assumed for all steel beams and columns.

\section{Residual Strength Ratio $\kappa$ and Ultimate Rotation Capacity $\theta_{u}$}

The rate of cyclic deterioration depends on the development of local instabilities. On this basis, four ranges of deterioration can be considered (Lignos \& Krawinkler, 2010a): The first range where local instabilities have not yet developed or are insignificant; the second range where local instability constantly increases; the third range corresponding to stabilization in buckle size; and finally the fourth range associated with crack propagation at local buckles and ductile failure. The third range is associated with residual strength with a low rate of deterioration. Lignos and Krawinkler (2010a) suggested a residual strength of approximately 0.4 for W-sections with $d \geq 21$ in based on the existing experimental data. In this study, a residual strength ratio equal to 0.4 was assumed for all steel beams and columns.

In the Modified Ibarra-Krawinkler model, the ultimate rotation capacity $\theta_{u}$ captures the brittle failure due to rapid propagation of cracks associated with severe local buckling. The Ultimate rotation capacity $\theta_{u}$ depends on the loading history such that for a loading history with few large cycles, large ultimate rotation capacity can be obtained. For a monotonic type of loading almost three times the rotation corresponding to symmetric cyclic loading protocols can be obtained. 
Lignos and Krawinkler (2010a) suggested ultimate rotation capacity $\theta_{u} 0.05$ to 0.06 for Wsection beams (no RSB) based on available experimental data.

\subsubsection{Axial Load- Bending Moment Interaction in Columns}

In order to provide a realistic tracking of initiation of yielding in axial load and bending moment, P-M interaction should be properly considered in the analysis procedure. Distributed plasticity models, fiber section and Finite mesh element models, are capable of capturing Axial loadbending moment interaction directly. The concentrated plasticity models may capture axial load and employ axial load-bending moment (P-M) yield surfaces to capture moment-rotation response. Alternatively, average values of the axial loads during nonlinear response may be considered to capture moment-rotation response.

In this study to consider the effect of axial load-bending moment (P-M) on estimating the moment capacity of the column in the presence of the axial load, the bending strength of the column was reduced based on equations provided by ANSI/AISC 360-10 (AISC, 2010b) as shown below:

For $\frac{P_{r}}{P_{c}} \geq 0.2, \quad \frac{P_{r}}{P_{c}}+\frac{8}{9}\left(\frac{M_{r x}}{M_{c x}}+\frac{M_{r y}}{M_{c y}}\right) \leq 1.0$ (AISC 360-10 equation H1-1a)

For $\frac{P_{r}}{P_{c}}<0.2, \quad \frac{P_{r}}{2 P_{c}}+\left(\frac{M_{r x}}{M_{c x}}+\frac{M_{r y}}{M_{c y}}\right) \leq 1.0$ (AISC 360-10 equation H1-1b)

Where $P_{r}, M_{r x}$ and $M_{r y}$ are axial, strong and weak bending moments acting on the column and $P_{c}, M_{c x}$ and $M_{c y}$ are axial, strong and weak bending moments strength of the column. A constant

$\frac{P_{r}}{P_{c}}$ ratio for a column was estimated based on the axial load due to gravity loads plus an average of axial loads acting on the column during nonlinear pushover analysis using ASCE 7-10 wind loading. Then by using the above equations the moment strength was reduced accordingly. 
For a box column, the effect of axial loads on moment-rotation relationship parameters such as pre-capping plastic rotation, $\theta_{p}$, post-capping rotation, $\theta_{p c}$, and reference cumulative plastic rotation, $\Lambda$, is directly incorporated in terms of axial load ratio $\frac{N}{N_{y}}$ into the equations provided by Lignos and Krawinkler (2010b). However, for a W-section column no specific recommendations are developed by the study to incorporate the effect of axial loads on the moment-rotation relationship parameters. Therefore, in this study the pre-capping plastic rotation, $\theta_{p}$, and postcapping rotation, $\theta_{p c}$, were reduced by a reduction factor obtained from ASCE/SEI 41-13 as shown in Table 2-2 As shown in the table, the pre-capping and post-capping plastic rotations recommended by ASCE/SEI 41-13 for various column flange and web slenderness ratios are compared and the reduction factors according to the axial load ratio are obtained. No reduction in reference cumulative plastic rotation, $\Lambda$, was assumed for columns due to axial loads, since no valid related recommendation was found in literature.

Table 2-2. Determining the reduction factor for the axial load effect on pre-capping and precapping plastic rotations according to ASCE/SEI 41-13

\begin{tabular}{|c|c|c|c|c|c|c|}
\hline & \multirow{2}{*}{$\begin{array}{c}\begin{array}{c}\text { For beam } \\
\left(P_{r}=0\right)\end{array} \\
\begin{array}{c}\text { Specified } \\
\text { value }\end{array}\end{array}$} & \multicolumn{2}{|c|}{ For $\frac{P_{r}}{P_{c}} \leq 0.2$} & \multicolumn{2}{|c|}{ For $0.2 \leq \frac{P_{r}}{P_{c}} \leq 0.5$} \\
\hline & & & $\begin{array}{l}\text { Specified } \\
\text { value }\end{array}$ & $\begin{array}{l}\text { Reduction } \\
\text { Factor }\end{array}$ & Specified value & Reduction Factor \\
\hline 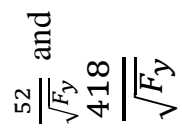 & $\theta_{p}$ & $9 \theta_{y}$ & $9 \theta_{y}$ & 1.0 & $11\left(1-\frac{5}{3} P / P_{C L}\right) \theta_{y}$ & $\frac{11}{9}\left(1-\frac{5}{3} P / P_{C L}\right)$ \\
\hline 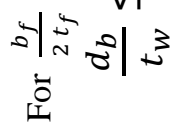 & $\theta_{p c}$ & $11 \theta_{y}$ & $11 \theta_{y}$ & 1.0 & $17\left(1-\frac{5}{3} P / P_{C L}\right) \theta_{y}$ & $\frac{17}{11}\left(1-\frac{5}{3} P / P_{C L}\right)$ \\
\hline 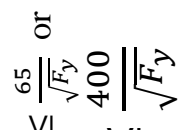 & $\theta_{p}$ & $4 \theta_{y}$ & $4 \theta_{y}$ & 1.0 & $1 \theta_{y}$ & $\frac{1}{4}$ \\
\hline 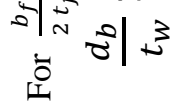 & $\theta_{p c}$ & $6 \theta_{y}$ & $6 \theta_{y}$ & 1.0 & $1.5 \theta_{y}$ & $\frac{1}{4}$ \\
\hline
\end{tabular}




\subsubsection{Composite Action Effect On Response of Beams}

Typically, steel structures include a composite slab roofing system. The composite action developed by the interaction between concrete slab and steel beam results in changes in the response of the beam by increasing the stiffness, shifting of neutral axis and asymmetrically increasing moment strength. In the positive moment direction, the composite action and the associated restraint which controls local and lateral torsional buckling results in significantly higher bending strength. However, it also results in a higher tensile strain in the bottom flange and connection components. In the negative moment direction, it may result in early lateraltorsional buckling (PEER/ATC, 2010).

As shown in Figure 2-16, in the 47-story building the secondary beams are leveled to the bottom of the moment resisting beams and the slab is attached to the web of the beams in the moment frame. Thus composite action for moment resisting beams is not provided in the 47-story building. For the two other buildings, the 30- and 40-story buildings, no composite action for beams in moment frames was assumed. However, in all three building models the effect of composite action for both gravity beams and their connections was included. For gravity beams the stiffness of composite beams was considered. The effects of composite action in the response of gravity connections were incorporated, as discussed in a later section.

\subsubsection{Modeling of Steel Moment-Resisting Connections}

The type of beam-column connection designed to transfer forces between beams and columns can significantly affect the response of the structure. Generally, steel connections, based on their ability to transfer bending moment between beams and columns, are classified into three categories: (a) Simple; (b) Partially Restrained (PR); and (c) Fully restrained (FR) connections. Gravity connections, such as: shear tab; seat and top angles; and double web angle connections, are typically assumed as simple connections where the moment transfer is neglected. However, 
experiments have shown substantial rotational strength and stiffness of shear tab connections, particularly when composite action with a concrete deck is provided (FEMA, 2000b; Liu \& Astaneh-Asl, 2000). The contribution of gravity frames to lateral stiffness and strength can be essential in large displacements where the moment-resisting frame system response experiences negative tangent stiffness. The effect of gravity frames may result in shifting of the displacement corresponding to the onset of lateral instability of the structure. Therefore, the rotational strength and stiffness of composite gravity connections were considered in the current study. The details and assumptions of modeling the gravity frames are described in a later section.

Steel beam to column connections with partial rotational stiffness or partial rotational strength or both, are considered Partially Restrained (PR) connections such as: Extended end plate connections; Bolted flange plate connections; T-stub connections; and Double flange angle connections. PR connections usually show significant rotation within the connection before the connection develops its ultimate resistance. These connections are mainly bolted connections which became more common after the Northridge earthquake as an alternative to welded-flangebolted-web connections.

Connections that develop relatively small rotation between beams and columns compared to rotations developed in beam end and panel zones are classified as Fully Restrained (FR) connections. Typically, no relative rotation in connection is assumed for FR connections. Welded flanges; Welded or Bolted Flange Plate, T-Stub; and Moment End-Plate connections are typical connections considered as FR connections. However, stiff PR connections also may be considered as FR connections.

In the Northridge earthquake (1994), more than 150 welded steel moment frame structures sustained damage in their welded connections (Liu \& Astaneh-Asl, 2000). So it was shown that steel moment frames with prequalified welded beam-to-column connections are much more 
vulnerable to brittle damage than was thought. The most common damage was at the weld of the beam bottom flanges to the columns. It was concluded that the stability of a steel moment frame is highly related to connection capacity to survive intact during lateral loading. Concerns raised about the seismic performance of the steel moment frame led to the initiation of the SAC Joint Venture research program to address the issues related to the seismic performance of steel moment resisting structures. The research delivered several documents regarding evaluation and retrofitting pre-Northridge connection buildings as well as recommendations for design new steel moment frame structures.

All three high-rise buildings investigated in this research have fully restrained connections. Among them the 47-story building has pre-Northridge connection type which is fully discussed and its simulation is described in the following section. The two other high-rise buildings, 30 - and 40-story buildings, are not provided with connection detailing. Considering their framing type as intermediate moment frames, only rotational restriction to $0.02 \mathrm{rad}$ was implemented and it was assumed they can provide enough rotational strength up to the rotational limit.

\section{Pre-Northridge Connections Used in 47-story Building}

Welded-flange-bolted/welded-web connections, known as Pre-Northridge Connections, are FR connections extensively used in seismic reigns since early 1970s due to of the greater economy of this type of connection and an expected satisfactory inelastic performance. After the Northridge earthquake (1994), damage survey reports indicated significant cracking and damage to weldedflange-bolted-web connections. The reports showed that the cracks mainly started at bottom flange welds and in a few cases it progressed through the column flange into the panel zone (FEMA, 2000b). The observation also showed that older buildings (before 1975) were less susceptible to connection cracking and in fact buildings designed after 1990 were the most 
vulnerable to connection damage (FEMA, 2000b). It was also shown that the frames with deeper beams experienced more significant connection cracking.

Pre-Northridge connections typically provide large enough rotational stiffness so it does not require any special consideration in the global structure analysis to account for flexibility of the connections (FEMA, 2000b). Therefore, for an accurate analytical simulation the resistance and rotational ductility of the connections should only be precisely accounted for in a nonlinear structural analysis. The resistance and ductility of the connections mainly depend on the failure modes. When the failure mode is associated with yield mechanisms, significant plastic rotational capacity can be achieved, while brittle modes of failure such as weld fracture leads to less ductility, energy dissipation and rotational capacity. Flexural yielding at end of the beam as well as the shear yield of panel zones is acceptable yield mechanisms that lead to large inelastic deformation in pre-Northridge connections. As illustrated in Figure 2-13 the following modes of failure are expected in structures using pre-Northridge connections:

- Fracture of Beam Flange Weld,

- Fracture at Weld Access Hole,

- Plastic Hinging of Beam,

- Beam Flange Buckling,

- Beam Web Buckling,

- Beam Lateral Torsional Buckling,

- Excessive Deformation of Panel Zone,

- Excessive Deformation of Column web and flange.

To determine the most probable failure mode(s), the vulnerability of the connection to each failure modes needs to be properly accessed. FEMA 355D (FEMA, 2000b), associated some equations of geometric parameters to define the vulnerability of connections to failure modes. 
Evaluating the failure modes and the associated equations for the pre-Northridge connection used in the 47-story building are discussed in following.

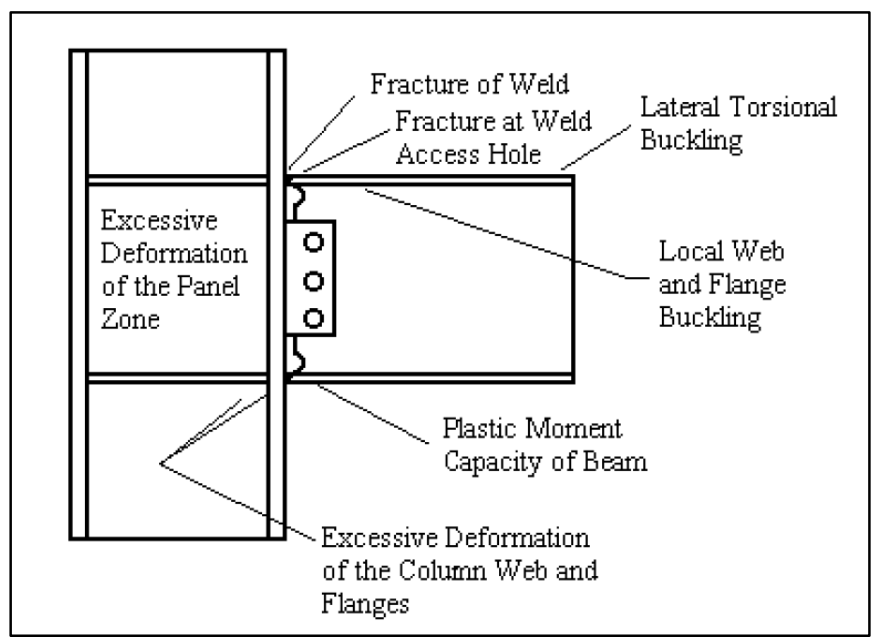

Figure 2-13. Failure modes of pre-Northridge connections (FEMA, 2000b)

The typical moment-resisting beam to column connection used in the 47-story building is shown in Figure 2-14. According to the detailing and the time of the construction, the connections are generally considered as pre-Northridge connections with the same expected modes of failure. To develop an accurate nonlinear analytical model incorporating all buildings characteristics, all modes of failure associated with pre-Northridge connection were first evaluated for 47-story building's connections and the most likely failure modes were directly and indirectly incorporated in the analytical model.

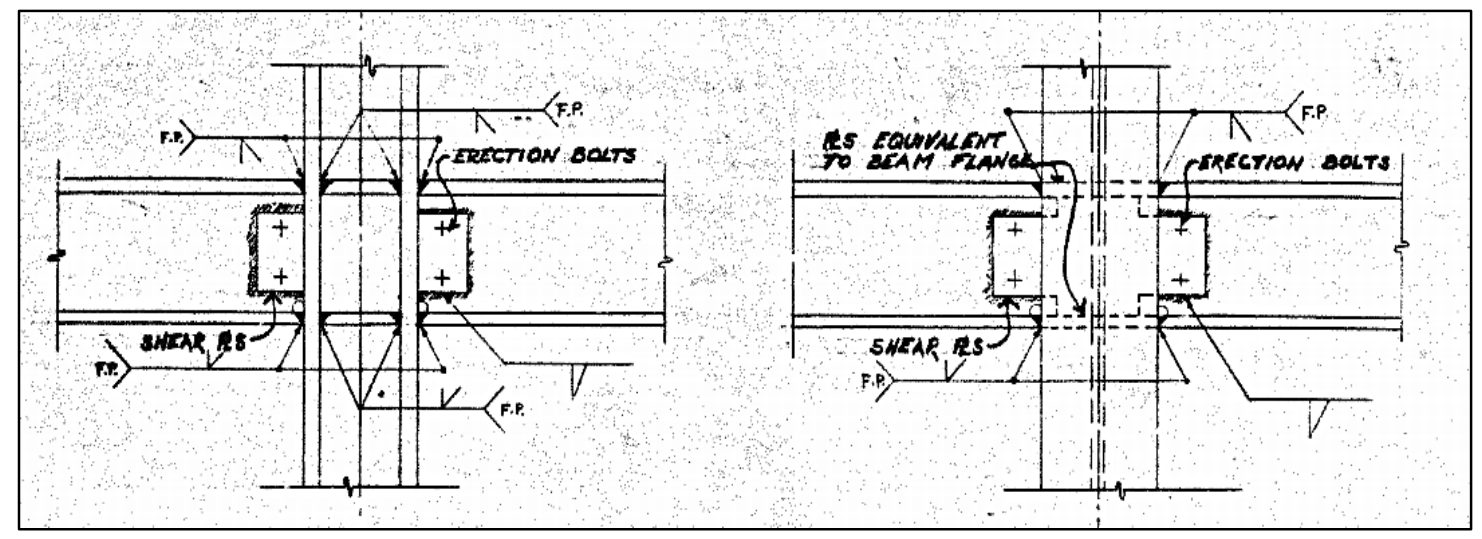

Figure 2-14. Typical beam to column moment-resisting connection in the 47-story building 
Among the mentioned potential modes of failure of pre-Northridge connections, two brittle failure modes of weld fracture and fracture of access hole are more specifically related to the preNorthridge connection which significantly restricts the rotational ductility of the connections. The other failure modes, related to elastic buckling or yielding in beams, columns, panel zones, and joint zones, are generally extendable to other connections. Among the expected failure modes, only beam plastic hinging and plastic distortion of panel zones are associated with desirable yield mechanisms and lead to appropriate energy dissipation and plastic ductility. In the following, all potential modes of failure for the 47-story building's pre-Northridge connections are evaluated using the equations provided in FEMA 355D.

1. Fracture of Beam Flange Weld or Weld Access Hole: Pre-Northridge welding using E70T-4 filler was typically associated with very low notch toughness. Also, leaving the backing bar in place as a routine practice in Pre-Northridge welding led to hiding the weld flaws. These facts made the Pre-Northridge welding susceptible to crack growth (FEMA, 2000b). It was also shown that the finish and geometry of the weld access hole in Pre-Northridge welding initiated the fracture of the beam flange. The failure modes associated with fracture in welding and fracture of weld access holes are not predictable. FEMA 355D recommends removing the backing bars and reinforcing welding to avoid this brittle failure. In this study, these types of failure are incorporated by limiting the rotational capacity of the pre-Northridge connections in the moment-rotation relationship according to recommendations provided in FEMA 355D.

2. Plastic Hinging of Beam: The inelasticity in the end of the beams is directly considered in analytical modeling in terms of concentrated plastic hinges. The concept and the approach of inelasticity modeling of beams and columns are discussed in detail in previous sections. 
3. Beam Flange, Web and Lateral Torsional Buckling: In steel structural design, the flange slenderness ratio $\frac{b_{f}}{2 t_{f}}$ and web slenderness ratio $\frac{d_{b}}{t_{w}}$ are used to determine the vulnerability to flange and web buckling respectively. FEMA 355D recommended the requirements of $\frac{b_{f}}{2 t_{f}} \leq \frac{52}{\sqrt{F_{y}}}$ and $\frac{d_{b}}{t_{w}} \leq \frac{418}{\sqrt{F_{y}}}$, the ductility requirements for special steel moment frames, to prevent elastic bucking in beam web and flanges. While the beam web and flange slenderness was already considered in development of the beam nonlinearity parameters as mentioned in the modified Ibarra-Krawinkler approach, the recommended requirements are checked as shown in Table 2-3. The table shows that for all beam cross sections used in moment resisting frame connected to columns with pre-Northridge connections, all beam flanges have acceptable slenderness, so no elastic flange buckling at the ends of the beams is expected. Checks of the web flange criteria as shown in Table 2-3 indicated that the beams do not satisfy the requirement and beam web buckling might be expected to occur. But considering other detailing used in the 47-story building construction resulted in a different conclusion. As shown in Figure 2-15, in momentresisting beams, transverse stiffeners had been considered with shorter spacing at critical section at the beam ends. Figure 2-16 shows the flooring system detail used in the 47story building. It is shown that the floor deck is connected to beam webs and provides a lateral support, halving the unbraced depth of the beams, which significantly reduces the web slenderness. Considering the transverse stiffeners and the lateral support and attached connection detailing of floor deck, it was concluded that beam web buckling is not expected for the 47-story building. 
Table 2-3. Checking the beam web and flange buckling failure mode for the 47-story building

\begin{tabular}{|c|c|c|c|c|c|c|c|c|c|c|}
\hline Beam Sections & $d($ in $)$ & $b_{f}($ in $)$ & $t_{f}(\mathrm{in})$ & $t_{w}(\mathrm{in})$ & $\frac{b_{f}}{2 t_{f}}$ & $\frac{52}{\sqrt{F_{y}}}$ & $\begin{array}{l}\text { Flange } \\
\text { check }\end{array}$ & $\frac{d_{b}}{t_{w}}$ & $\frac{418}{\sqrt{F_{y}}}$ & $\begin{array}{l}\text { Web } \\
\text { check }\end{array}$ \\
\hline I-A & 34.25 & 6.00 & 0.63 & 0.25 & 4.76 & 7.35 & OK & 137.00 & 59.11 & Not OK \\
\hline I-B & 34.50 & 6.00 & 0.75 & 0.25 & 4.00 & 7.35 & OK & 138.00 & 59.11 & Not OK \\
\hline I-C & 34.75 & 6.00 & 0.88 & 0.25 & 3.41 & 7.35 & OK & 139.00 & 59.11 & Not OK \\
\hline I-D & 35.00 & 6.00 & 1.00 & 0.25 & 3.00 & 7.35 & OK & 140.00 & 59.11 & Not OK \\
\hline I-E & 35.25 & 6.00 & 1.13 & 0.25 & 2.65 & 7.35 & OK & 141.00 & 59.11 & Not OK \\
\hline I-F & 35.50 & 6.00 & 1.25 & 0.25 & 2.40 & 7.35 & OK & 142.00 & 59.11 & Not OK \\
\hline I-G & 35.75 & 6.00 & 1.38 & 0.25 & 2.17 & 7.35 & OK & 143.00 & 59.11 & Not OK \\
\hline I-H & 49.38 & 6.00 & 0.69 & 0.38 & 4.35 & 7.35 & OK & 129.95 & 59.11 & Not OK \\
\hline I-I & 49.63 & 6.00 & 0.81 & 0.38 & 3.70 & 7.35 & OK & 130.61 & 59.11 & Not OK \\
\hline I-J & 49.88 & 6.00 & 0.94 & 0.38 & 3.19 & 7.35 & OK & 131.26 & 59.11 & Not OK \\
\hline I-K & 50.13 & 6.00 & 1.06 & 0.38 & 2.83 & 7.35 & OK & 131.92 & 59.11 & Not OK \\
\hline I-L & 50.38 & 6.00 & 1.19 & 0.38 & 2.52 & 7.35 & OK & 132.58 & 59.11 & Not OK \\
\hline I-M & 50.63 & 6.00 & 1.31 & 0.38 & 2.29 & 7.35 & OK & 133.24 & 59.11 & Not OK \\
\hline I-N & 50.50 & 7.00 & 1.25 & 0.38 & 2.80 & 7.35 & OK & 132.89 & 59.11 & Not OK \\
\hline $\mathrm{I}-\mathrm{O}$ & 50.50 & 8.00 & 1.25 & 0.38 & 3.20 & 7.35 & OK & 132.89 & 59.11 & Not OK \\
\hline I-P & 50.75 & 8.00 & 1.38 & 0.38 & 2.90 & 7.35 & OK & 133.55 & 59.11 & Not OK \\
\hline I-Q & 51.50 & 8.00 & 1.75 & 0.38 & 2.29 & 7.35 & OK & 135.53 & 59.11 & Not OK \\
\hline I-R & 52.00 & 12.00 & 2.00 & 0.63 & 3.00 & 7.35 & OK & 82.54 & 59.11 & Not OK \\
\hline I-S & 52.00 & 11.00 & 2.00 & 0.63 & 2.75 & 7.35 & OK & 82.54 & 59.11 & Not OK \\
\hline I-T & 52.00 & 13.00 & 2.00 & 0.63 & 3.25 & 7.35 & OK & 82.54 & 59.11 & Not OK \\
\hline I-U & 52.00 & 14.00 & 2.00 & 0.63 & 3.50 & 7.35 & OK & 82.53968 & 59.1 & Not OK \\
\hline $\mathrm{I}-\mathrm{V}$ & 51.13 & 7.00 & 1.56 & 0.38 & 2.24 & 7.35 & OK & 134.5526 & 59.1 & Not OK \\
\hline I-W & 51.50 & 8.00 & 1.75 & 0.38 & 2.29 & 7.35 & OK & 135.5263 & 59.1 & Not OK \\
\hline
\end{tabular}




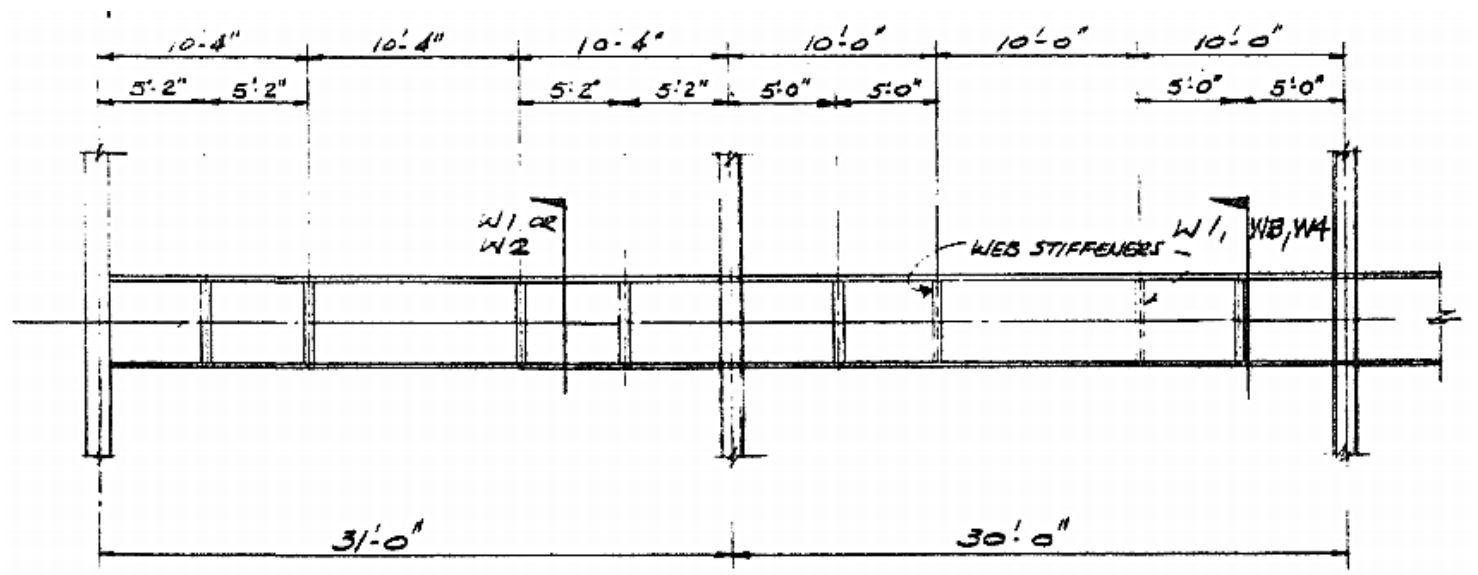

TYPICAL CORNER BAY TYPKAL INERTOR BAY

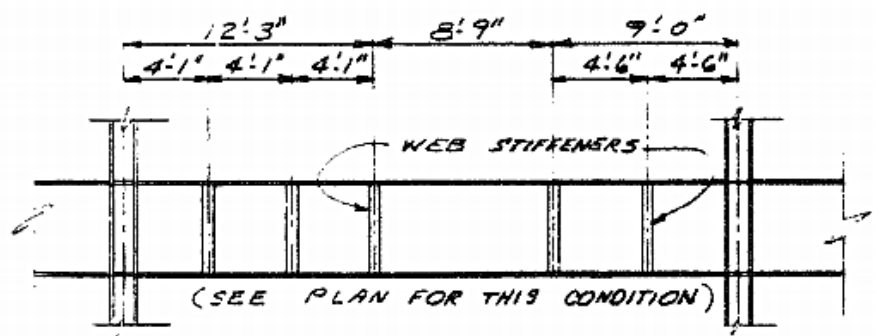

Figure 2-15. Web transverse stiffeners of beams in moment resisting frames of the 47-story building

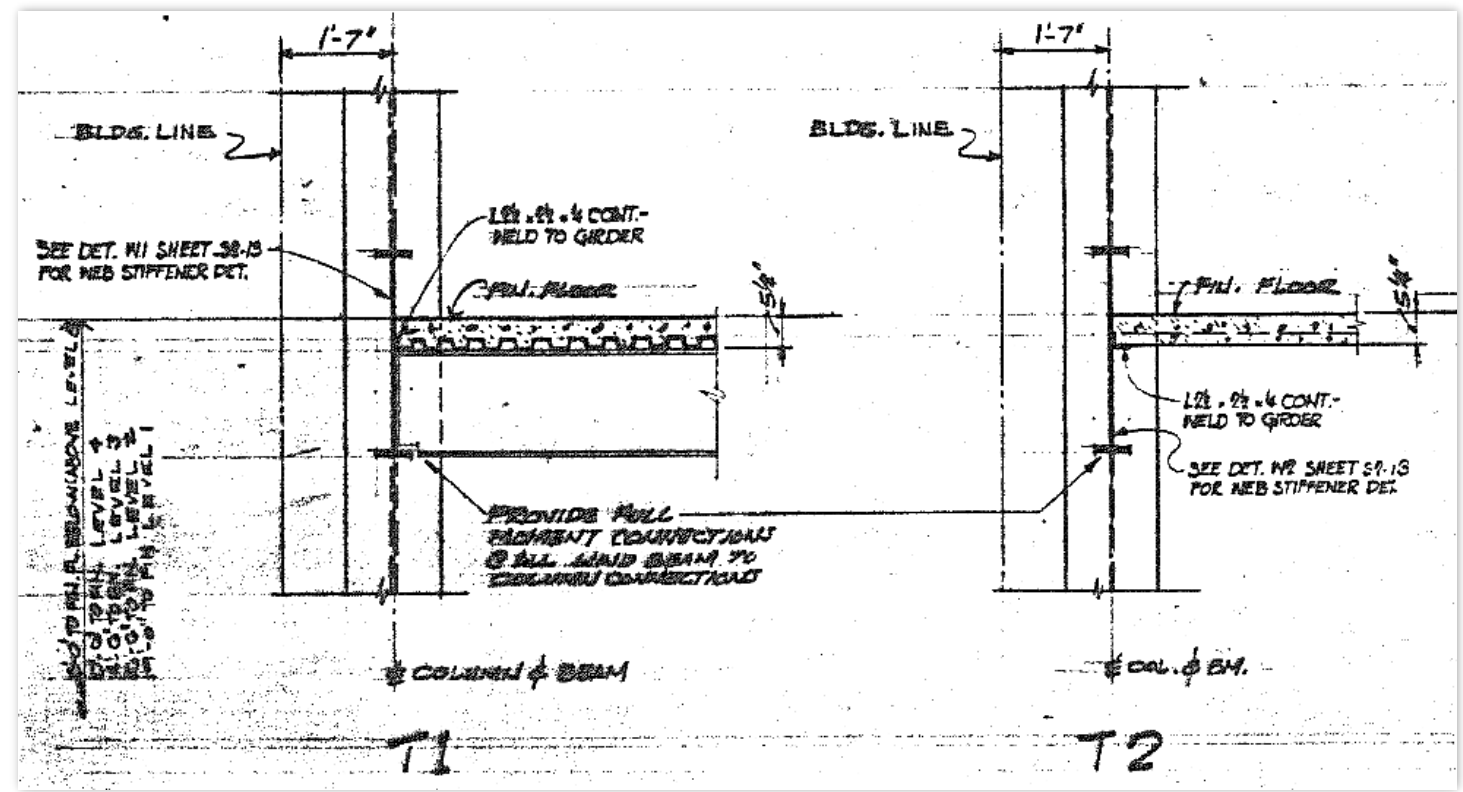

Figure 2-16. Details of flooring, framing and connections in the 47-story building 
4. Excessive Deformation of Panel Zone: shear plastic distortion of panel zones provides considerable ductility for the structure. Recent building codes emphasized the use of this ductility in seismic regions where higher ductility is demanded. However, in structures with pre-Northridge connections, the excessive deformation of panel zones and the consequent high stress developed in column flanges may cause the propagation of cracks initiated in the weld zone to the column. It eventually results in column damage, the most prohibited failure mode in seismic design. In this study, plastic distortion in panel zones is precisely modeled using a parallelogram model as described in the following section. The response of the panel zones in wind nonlinear time-history analysis and the resulting limited plastic distortion led to the conclusion that this mode of failure is not of concern.

5. Excessive Deformation of Column web and flange: when the beam reaches a high moment above its plastic capacity, it applies large localized forces to the columns. At the connection of the tension flange, a large concentrated force makes the column flange bend. In the absence of continuity plates, it results in a high concentration of flange stresses close to column web. This stress concentration leads to flange distortion and column web yielding/crippling which eventually causes column fracture initiation. FEMA 355D adopted an AISC seismic design requirement for determining the necessity for a continuity plate in connection design as shown below:

$t_{f c} \geq 0.4 \sqrt{\frac{P_{b f}}{F_{y c}}}, P_{b f}=1.8 t_{f b} b_{f b} F_{y b}$

where $t_{f c}$ is the thickness of column flange, $t_{f b}$ and $b_{f b}$ are the thickness and width of beam flange respectively. $F_{y c}$ and $F_{y b}$ are the steel yielding stresses of columns and beams respectively. In the 47-story building continuity plates with the same thickness of the beam flanges are used. Therefore, column failure due to excessive deformation in the web and flange is not a concern for the 47 -story building. 
Table 2-4 summarizes the assessment of all possible failure modes for pre-Northridge connections used in the 47-story building. It is also shown how the expected failure modes are incorporated into the analytical model. The table shows that the failure modes associated with local and torsional buckling of the beam as well as the mode of failure corresponding to excessive deformation of the column web and flange are not a concern for the 47-story building's pre-Northridge connections. The modes of failure of beam plastic deformation of beam and panel zone are directly incorporated into the analytical model. Finally, the brittle failure modes associated with fracture of welds and weld access holes are incorporated into the analytical model by restricting the rotational capacity of the connection based on the FEMA 355D recommendations as described in the following section. 
Table 2-4. Summary of modes of failure of the pre-Northridge connections, their controlling parameters and their consideration in the analytical analysis.

\begin{tabular}{|c|c|c|}
\hline Failure Mode & $\begin{array}{c}\text { Parameters to Control Failure Modes } \\
\text { According to FEMA 355D }\end{array}$ & How it was incorporated in analytical model \\
\hline $\begin{array}{l}\text { Fracture of Beam } \\
\text { Flange Weld }\end{array}$ & Not predictable & $\begin{array}{l}\text { Incorporated by limiting the rotational } \\
\text { capacity of the connection according to limited } \\
\text { rotational capacity recommended in FEMA } \\
\text { 355D }\end{array}$ \\
\hline $\begin{array}{l}\text { Fracture at Weld } \\
\text { Access Hole }\end{array}$ & Not predictable & $\begin{array}{l}\text { Incorporated by limiting the rotational } \\
\text { capacity of the connection according to limited } \\
\text { rotational capacity recommended in FEMA } \\
\text { 355D }\end{array}$ \\
\hline $\begin{array}{l}\text { Plastic Hinging } \\
\text { of Beam }\end{array}$ & $M_{p}=Z F_{y b}$ & $\begin{array}{l}\text { It was directly considered as beam plastic } \\
\text { hinges at two ends of the beam }\end{array}$ \\
\hline Flange Buckling & controlled if $\frac{b_{f}}{2 t_{f}} \leq \frac{52}{\sqrt{F_{y}}}$ & $\begin{array}{l}\text { Fully controlled and not required any } \\
\text { consideration }\end{array}$ \\
\hline Web Buckling & controlled if $\frac{d_{b}}{t_{w}} \leq \frac{418}{\sqrt{F_{y}}}$ & $\begin{array}{l}\text { Fully controlled and not required any } \\
\text { consideration }\end{array}$ \\
\hline $\begin{array}{l}\text { Lateral Torsional } \\
\text { Buckling }\end{array}$ & controlled if $L_{b}<\frac{2500 r_{y}}{F_{y}}$ & $\begin{array}{l}\text { Fully controlled by rigid deck and not required } \\
\text { any consideration }\end{array}$ \\
\hline $\begin{array}{c}\text { Excessive } \\
\text { Deformation of } \\
\text { Column web and } \\
\text { flange }\end{array}$ & $\begin{array}{l}\text { Continuity plates required if } \\
\qquad \begin{array}{c}t_{f c} \geq 0.4 \sqrt{\frac{P_{b f}}{F_{y c}}}, P_{b f}= \\
1.8 t_{f b} b_{f b} F_{y b}\end{array}\end{array}$ & $\begin{array}{l}\text { Fully controlled by continuity plates and not } \\
\text { required any consideration }\end{array}$ \\
\hline
\end{tabular}

\section{Rotational Capacity of Pre-Northridge Connections}

Generally, the ductility of a moment resisting frame structure is highly influenced by the rotational capacity of its connections. Although it is generally known that the pre-Northridge connections provide a limited rotational capacity, the evaluation of the rotational capacity of these connections is difficult since the brittle failure modes associated with weld fracture highly restrict its rotational capacity, and were not in consideration in the design stage. Based on the existing test data on pre-Northridge connections, FEMA 355D provided suggestions for predicting the rotational capacity of pre-Northridge connections. 
According to FEMA 355D, two rotational limits are considered: the plastic rotation at initial failure $\theta_{p}$; and the plastic rotation at final Failure $\theta_{g}$.

The plastic rotation at initial failure $\theta_{p}$ is defined as the plastic rotation where either fracture occurred or the strength drops to below $80 \%$ of the plastic capacity. FEMA 355D conducted least squares evaluation of the rotational capacity on the two following sets of experimental data: (1) Type 1: pre-Northridge connections with older E70T-4 welds and steels with lower yield to tensile stress ratio; and (2) Type 2: more recent pre-Northridge connections that were welded with an E70T-4 electrode and with steel with larger yield to tensile stress ratio. The following mean and standard deviation of plastic rotation at initial failure $\theta_{p}$ were suggested for two types of preNorthridge connections:

For Type 1 pre-Northridge connections:

$$
\begin{gathered}
\theta_{p_{\text {mean }}}=0.051-0.0013 d_{b} \\
\sigma_{p}=0.0044-0.0002 d_{b}
\end{gathered}
$$

For Type 2 pre-Northridge connections:

$$
\begin{gathered}
\theta_{p_{\text {mean }}}=0.011 \text { radians } \\
\sigma_{p}=0.007 \text { radians }
\end{gathered}
$$

where $\theta_{p_{\text {mean }}}$ and $\sigma_{p}$ are mean and standard deviation of initial failure rotational capacity respectively, and $d_{b}$ is the beam depth.

The distinction between rotational capacities suggested for the two mentioned types of preNorthridge connection is mainly attributed to increase in steel yielding to tensile strength ratio over time. It indicates the fact that the older buildings with lower yielding stress showed less frequency of damage. The negative effect of larger beam sizes on rotational capacity is also 
shown in the equation suggested for type 1 connections, while for type 2 , due to relatively small variation in beam depth, the effect is not included. It should be noticed that the recommended equations can be used with regard to the range of beam size of the experimental database. The report recommends the use of Type 1 equations for frames with W24 or lighter beam framing and Type 2 equations for heavier framing beam sizes up to W36 sections.

The second rotational capacity limit, Ultimate rotational capacity, $\theta_{g}$, is referred to rotation where the connection will not be able to support the gravity loads anymore. FEMA 355D suggests the following equations to determine the mean and standard deviation of ultimate rotational capacities for all type of pre-Northridge connections:

$$
\begin{gathered}
\theta_{g_{\text {mean }}}=0.043-0.0006 d_{b} \\
\sigma_{g}=0.011+.0004 d_{b}
\end{gathered}
$$

This estimation involves significant uncertainties arising from the variation of gravity load with the age and type of construction and testing condition of available test data. While this rotational capacity technically corresponds to tearing or fracture of the shear tab, the conducted experimental testing barely continues to that extent. Experimental data have also shown that, after fracture of the second weld, the connection which is associated with significant strength drop is still able to provide $15 \%$ to $20 \%$ of beam plastic capacity and considerable shear resistance. It recommends that a residual strength equivalent to $15 \%$ to $20 \%$ of beam plastic capacity can be considered for connection rotation beyond this nominal ultimate rotational capacity.

ASCE 7-10-13 "Seismic Rehabilitation of Existing Buildings" suggests a moment-rotation relationship for pre-Northridge connections by adopting the equations recommended in FEMA 355D. Figure 2-17 shows the suggested moment-rotation relationship using the mean initial and 
ultimate failure( $\theta_{p_{\text {mean }}}$ and $\theta_{g_{\text {mean }}}$ ) recommended by FEMA 355D for type 1 , older preNorthridge connection structures.

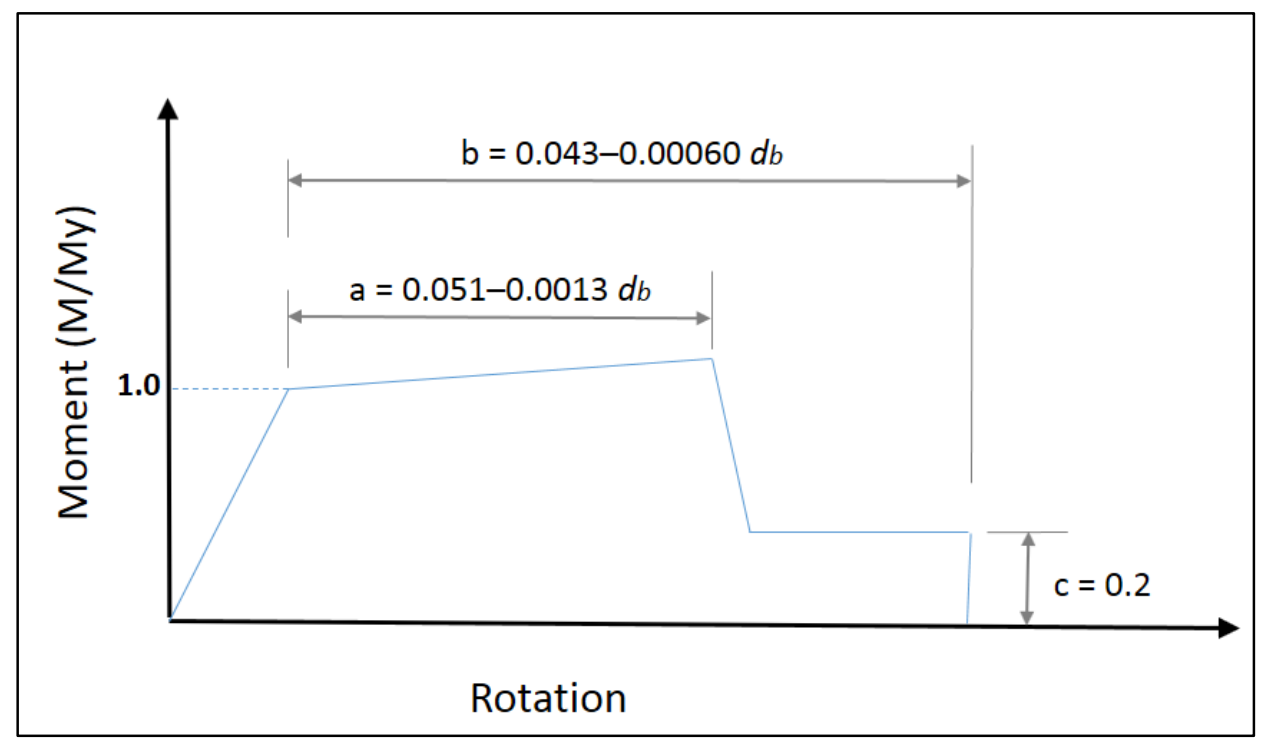

Figure 2-17. Nonlinear moment-rotation relationship suggested for pre-Northridge connections according to ASCE 41-13

Despite the general rotational limits suggested by FEMA 355D and ASCE41, which result in a symmetric moment-rotation relationship for pre-Northridge connection, the experimental results indicate an asymmetric response of pre-Northridge connections. The measured moment-rotation behavior of pre-Northridge connections in two experimental studies are shown in Figure 2-18. As shown in the figure the typical response of pre-Northridge connection is characterized by a sudden loss of strength and stiffness in response to positive moment due to fracture of the bottom flange weld. In a reverse moment, the weld crack of the bottom flange becomes fully closed which allows development of a relatively large moment resistance in negative moment response. Figure $2-18 \mathrm{~b}$ indicates that the cyclic moment-rotation response after initial fracture follows a pinching behavior. While the further cyclic response is not captured in these experimental studies, for larger cyclic loading the connection is expected to experience a strength drop in negative moment response due to fracture of the top flange welding. Then the connection response is 
characterized by a $15 \%$ to $20 \%$ residual strength provided by shear tap. The connection finally fails by tearing and fracture of the shear tap.

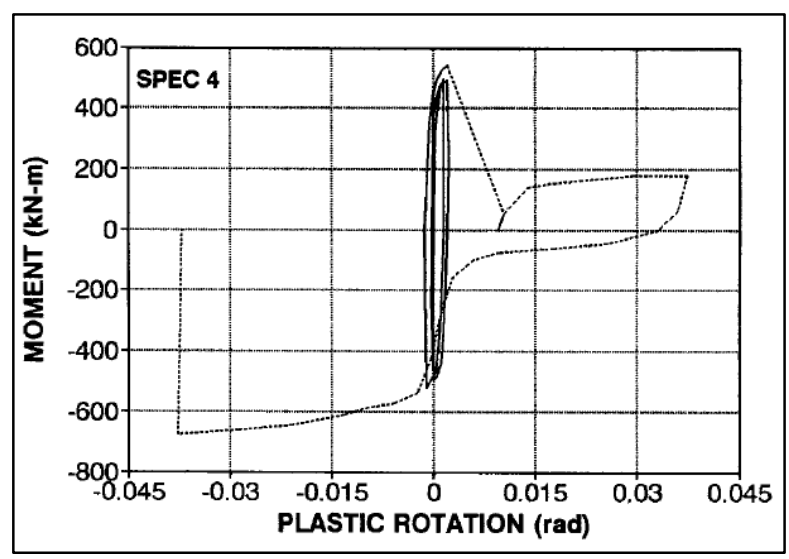

(a)

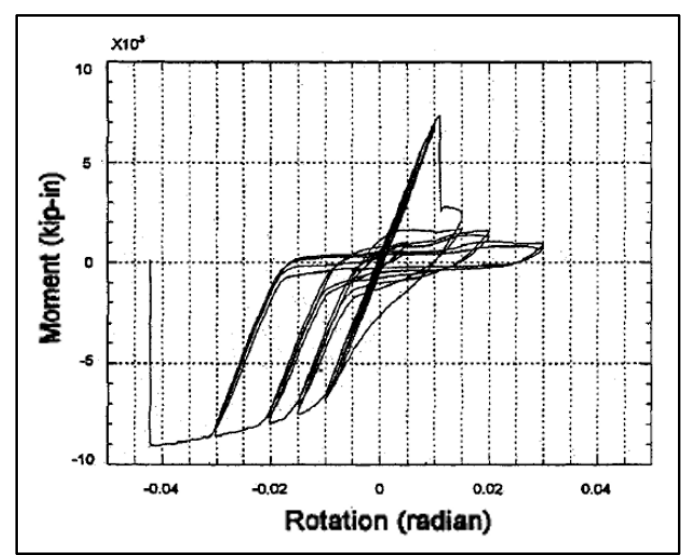

(b)

Figure 2-18. Instances of measured moment-rotation behavior of pre-Northridge connections in experimental tests: (a) conducted by Lee et al., (2000); (b) conducted by Engelhardt et al. (1993)

The hysteretic moment-rotation model developed and used in this study for the pre-Northridge connections of the 47-story building is shown in Figure 2-19a. The model follows the response characteristics obtained in experimental studies shown in Figure 2-19b. As shown in Figure 2-19, the model consists of a strength capacity backbone and a pinching hysteretic response. The backbone in the positive moment is characterized by peak strength equal to 1.1 times the plastic moment capacity of the beam, $\mathbf{M}_{\mathrm{pb}}$. The evaluation of failure modes of pre-Northridge connections of the 47-story building as summarized in Table 2-4, showed that, while all beam elastic buckling failure modes are controlled, plastic hinging at end of the beam is expected. Therefore, the peak strength is set as high as to allow the development of beam nonlinearity and associated hardening. The backbone in the positive moment then drops due to the fracture in the bottom flange weld at rotation equal to 0.011 radians. It then follows with residual strength equal to $20 \%$ of $\mathrm{M}_{\mathrm{pb}}$ until the final failure at 0.05 radians.

The backbone in negative moment corresponds to a peak strength equal to 1.1 times the plastic moment capacity of the beam, $\mathrm{M}_{\mathrm{pb}}$, allowing development of beam nonlinearity in the negative 
moment. The response in negative moment maintains the peak strength due to the crack closing mechanism while the reverse moment experiences a significant strength drop. The backbone then drops in rotation equal to 0.035 radians corresponding to fracture of top flange weld. The backbone then follows constant residual strength the same as for positive moment response.

Estimating the rotational limits corresponding to weld fractures for a 47-story building with large beam size was very challenging due to lack of experimental data for such deep beams. The FEMA 355D recommendations were also not applicable for the 47-story building, since the 47story building's beam size ranged from 34 to 52 inches, much beyond the recommended range for FEMA 355D equations (up to W24 for Type 1 and W36 for Type2 connections). Using engineering judgment and best available data, the rotation corresponding to initial failure was selected equal to 0.011 radians, equal to the mean value recommended by FEMA 355D for Type 2 (newer buildings) connections. This amount was selected because on the one hand the fracture rotation should be smaller due to the beam size effect and on the other hand the 47-story building is considered to be an older building and less vulnerable to damage. Thus, selecting rotation corresponding to initial failure equal to 0.011 radians was considered acceptable. The rotation corresponding to the form of second failure (top flange fracture) was selected equal to a mean plus a standard deviation of ultimate rotational capacity recommended by FEMA 355D. This amount for the beam size range of the 47 -story building was approximately equal to 0.035 radians. While this amount is defined as ultimate rotational capacity, considering beneficial effects of the floor slab on redistribution of gravity load to adjacent beams, the connection is expected to develop residual strength as considered in this model. The moment-rotation relationship for a pre-Northridge connection joining a W24x84 steel beam was developed based on the described model is shown in Figure 2-19. 


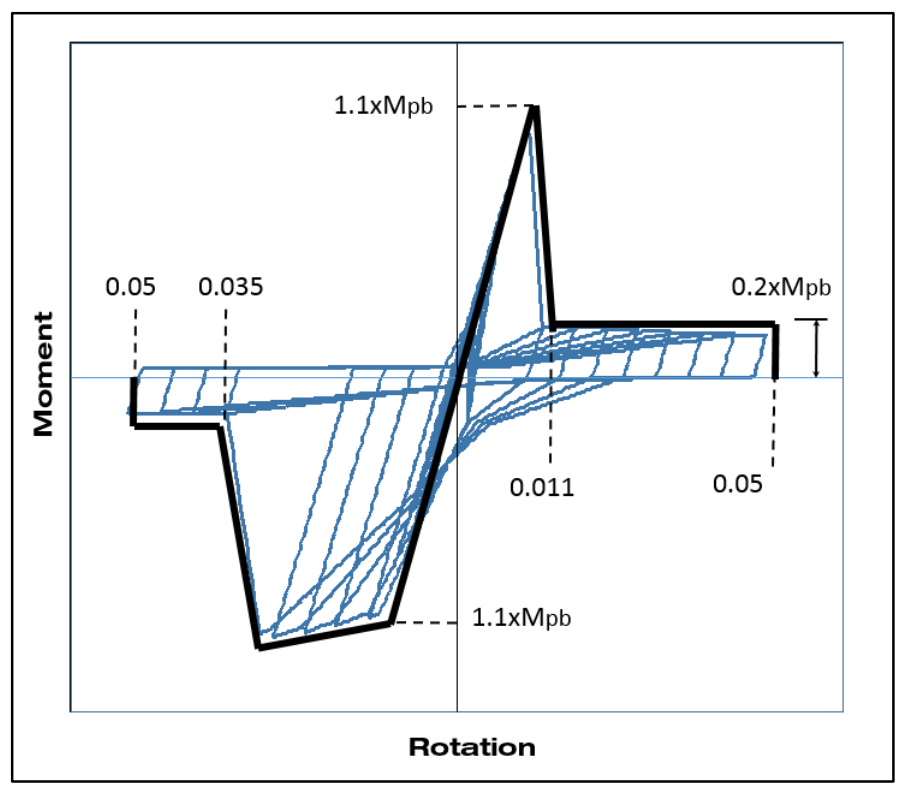

(a)

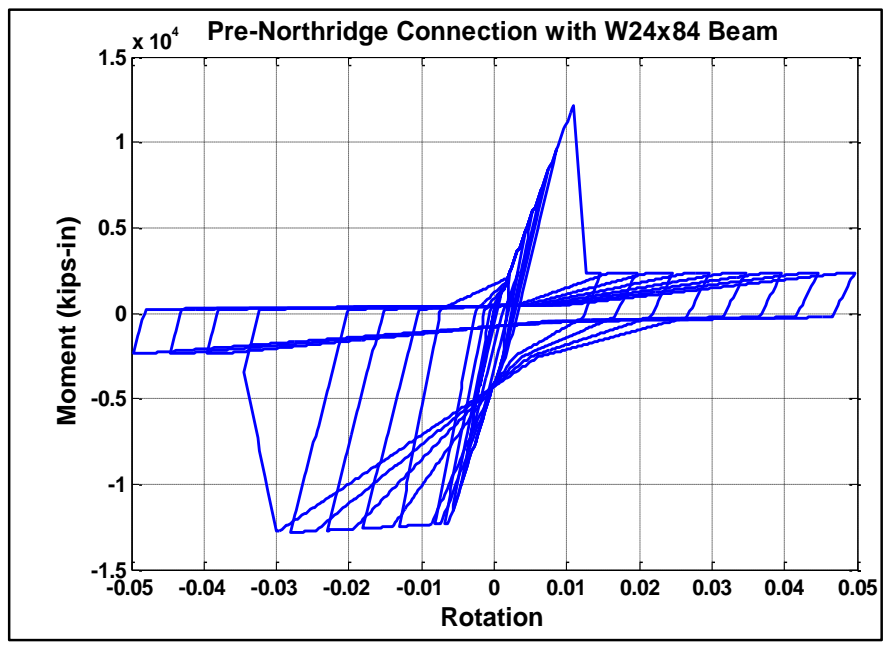

(b)

Figure 2-19. Hysteretic moment-rotation relationship of pre-Northridge connections: (a) developed idealized model; (b) moment-rotation relationship of a pre-Northridge connection with W24x84 beam

\subsubsection{Modeling The Nonlinearity of Steel Panel Zones}

In steel moment-resisting frames, the transfer of moment between beams and columns causes a complex stress and strain distribution in the beam-column joint. As shown in Figure 2-20a, the unbalanced moments in the beam-column joint cause high shear stresses in the panel zone and high normal stresses in a portion of the column flanges connected to beam flanges. Nonlinear 
shear distortion of a panel zone initiates via yielding of the column web at the two corners of the panel zone. The yielding then propagates to the whole panel zone region which allows the panel zone to deform to a parallelogram shape as shown in Figure 2-20b.

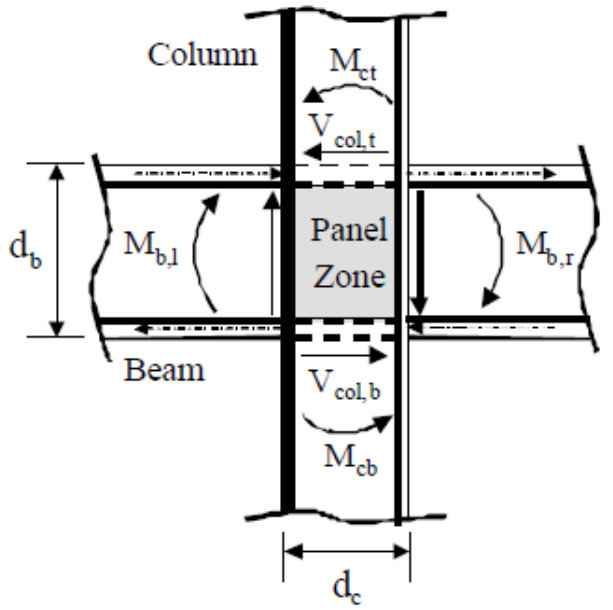

(a)

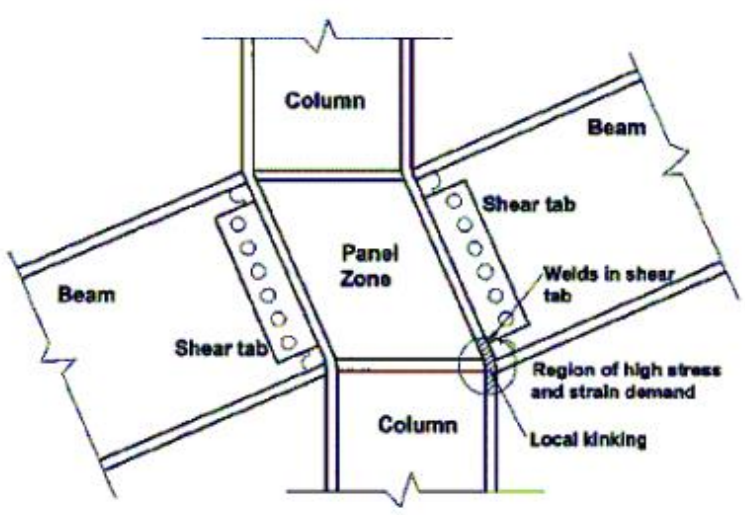

(b)

Figure 2-20. Panel zone forces and deformation: (a) moment and shear forces due to lateral loads, (b) panel zone distortion

The behavior of the panel zone plays a significant role in the overall response of the structure to lateral loads. It has been recognized that the shear yielding of the panel zone is an efficient source of ductility and energy dissipation while its hysteresis behavior is very stable. The typical shear hysteric behavior of panel zones is shown in Figure 2-20. It is shown that the hysteric behavior is generally characterized by large stable hysteresis loops, continuously increasing strength after first yielding. The experimental studies also showed the cyclic response of panel zones is associated with limited deterioration, unless due to buckling or fracture of a welded web doubler plate. Since shear buckling is avoided by code detailing criteria, cyclic deterioration can be neglected in the modeling of steel panel zones. 


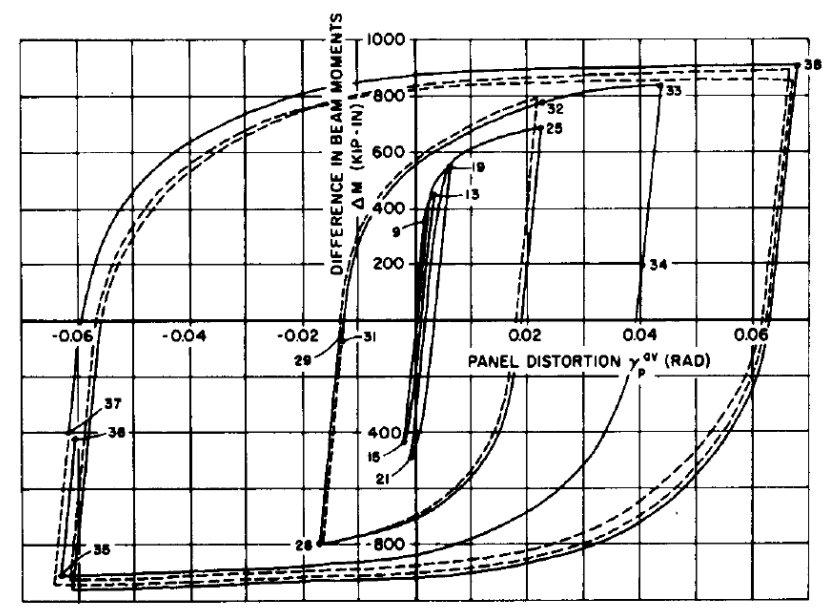

Figure 2-21.Cyclic shear behavior of a weak panel zone

According to the stable shear hysteretic behavior of a panel zone, the design codes, since UBC 1991, have permitted the use of weak panel zones which deform inelastically prior to reaching their design strength. It allows the panel zones to participate in energy dissipation and also provides a sort of controlled deformation capacity and higher ductility. However, the low strength and nonlinearity in panel zones decrease the strength and stiffness of the buildings. Experimental studies have shown that in welded beam-column connections excessive shear distortion of panel zones leads to severe local kinks at the beam and column flanges close to the four corners of the panel zone, eventually causing premature fracture in the joint (Krawinkler, 1978; Lin, Tsai, Kong, \& Hsieh, 2000; Popov, 1987). Therefore, excessive shear distortion of panel zones will have a detrimental effect on the ductility achieved by the nonlinearity of beams. It suggests that the strengths of the beam, columns, and panel zones need to be well proportioned to achieve optimum ductility.

Several mathematical models representing the behavior of panel zones have been developed (Jin \& El-Tawil, 2005; Kim \& Engelhardt M., 1995; Krawinkler, 1978; Tsai \& Popov, 1988). Typically, these models characterize the shear distortion of the panel zone by proposing shear load-shear distortion relationships based on experimental data or modifications to pre-existent 
models. Despite their difference in representation of inelastic behavior, they all agree on elastic response characteristics and the yield strength in shear $V_{y}$ and the elastic shear stiffness $K_{e}$ as expressed in following:

$$
\begin{gathered}
\mathrm{V}_{y}=\frac{F_{y}}{\sqrt{3}} A_{e f f}=\frac{F_{y}}{\sqrt{3}}\left(0.95 d_{c} t_{p}\right) \approx 0.55 F_{y} d_{c} t_{p} \\
\gamma_{y}=\frac{F_{y}}{\sqrt{3} \times G} \\
\mathrm{~K}_{e}=\frac{V_{y}}{\gamma_{y}}=0.95 d_{c} t_{p} G
\end{gathered}
$$

where $A_{e f f}$ is the effective shear area, and $d_{c}$ and $t_{p}$ are depth and web thickness of the column respectively. $\gamma_{y}$ is the yield distortion, and $\mathrm{G}$ is shear modulus of the column material.

A mathematical model of panel zones implemented in this study is the model proposed by Krawinkler (1978) and presented in PEER-ATC -72, and FEMA 355C (Krawinkler \& Venture, 2000). The model includes a trilinear monotonic shear force-shear distortion relationship as shown in Figure 2-22. The elastic control values of this model; the yield strength in shear $\boldsymbol{V}_{\boldsymbol{y}}$ and the elastic shear stiffness $K_{e}$ are the same as already described. In the second stage, after shear yielding of panel zone, the shear force - shear distortion relationship represents a significant increase in shear resistance which can be attributed to the bending resistance of the column flanges. The full plastic shear strength of the joint $V_{P}$ will be attained at a shear distortion value of $4 \gamma_{y} . V_{P}$ is estimated using the following equation:

$$
V_{p}=V_{y}\left(1+\frac{3 K_{p}}{K_{e}}\right) \approx 0.55 F_{y} d_{c} t_{p}\left(1+\frac{3 b_{c} t_{c f}^{2}}{d_{b} d_{c} t_{p}}\right)
$$

where $b_{c}$ and $t_{c f}$ are the width and thickness of the column flange respectively, and $K_{p}$ is the post-yielding shear stiffness of panel zone. In the last stage of the shear force-shear distortion 
relationship, the strength will be sustained beyond the shear distortion of $4 \gamma_{y}$ with an assumed strain-hardening.

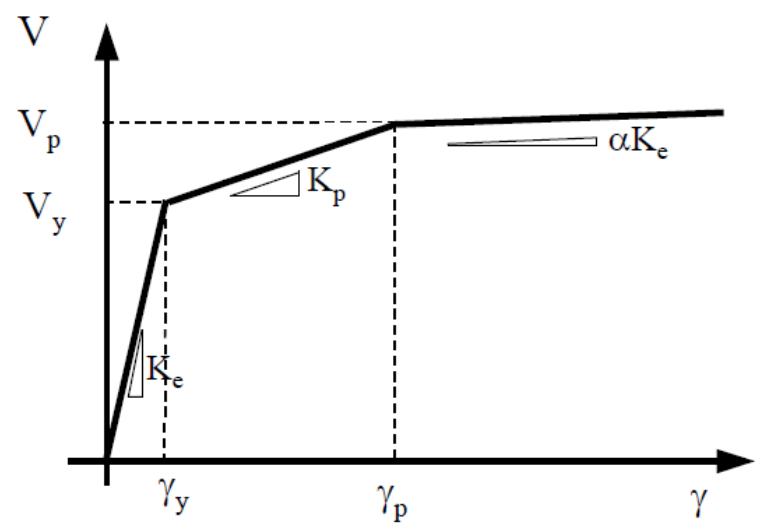

Figure 2-22. Trilinear shear force - shear distortion response of panel zones (Gupta and Krawinkler, 1999)

According to FEMA 355C, in frame analyses in which the numerical model utilizes line elements to model the beams and columns, the panel zones can be either modeled by a scissors model or a parallelogram model shown in in Figure 2-23a and 2-23b respectively. The scissors model is a simplified arrangement to represent the panel zone shear force- shear distortion in numerical models. As shown in Figure 2-22a, this model consists of two rigid elements extended to the panel zone dimensions and joined at the beam-column centerline. The rotation between two rigid elements is controlled by a rotational spring or two rotational springs where the used program includes only bilinear springs. Figure 2-23c illustrates two bilinear springs with shear forcedistortion relationships modeling the panel zone trilinear behavior. In scissors model, the assembly rotates according to moment difference in beams and columns related to panel zone shear. Therefore, the rotation of the spring is equivalent to the distortion of the panel zone. This model involves two approximations: (1) the shear force in the panel zone basically needs to be estimated based on the moment in the beams and columns at panel zone boundaries instead of the 
moment of beams and columns at the spring location, (2) the right angles between panel zone boundaries and beams and column elements are not maintained.

The parallelogram model is a more accurate model which avoids the approximations associated with scissors but it requires more modeling detail. As shown in Figure 2-23b, the model includes 8 rigid elements, three zero-stiffness hinges in three corners and a rotational spring (or two rotational springs) in the remaining corner. The assembly of these elements, hinges and the spring(s) can deform into a parallelogram to simulate the shear distortion of panel zones. The lateral deformation and consequent distortion response of the model are dominated by the characteristics of the rotational spring. 


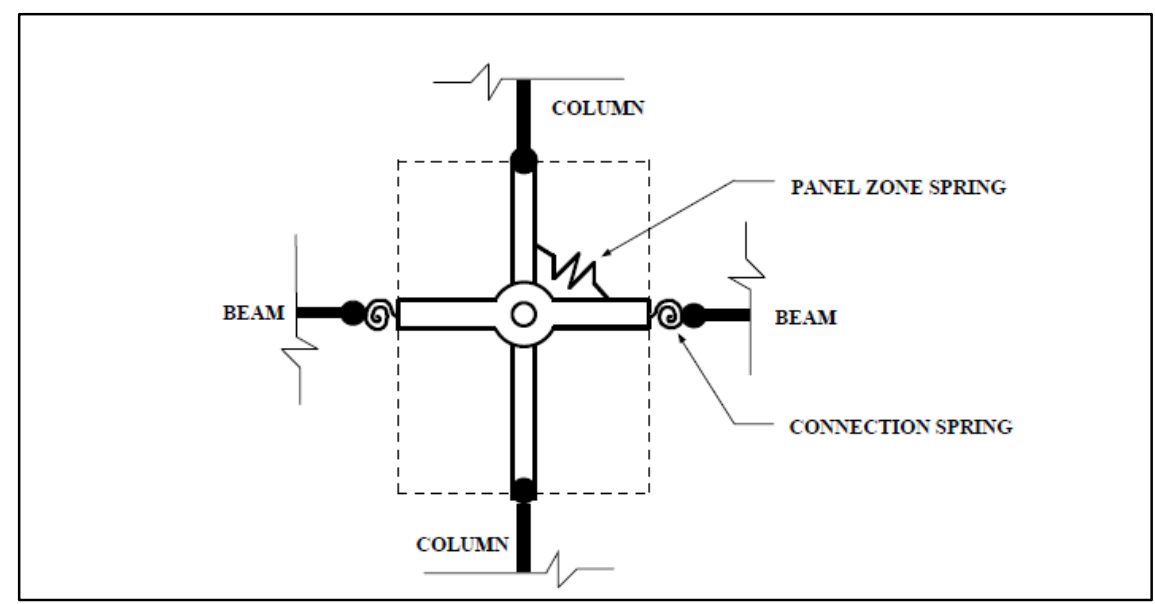

(a)

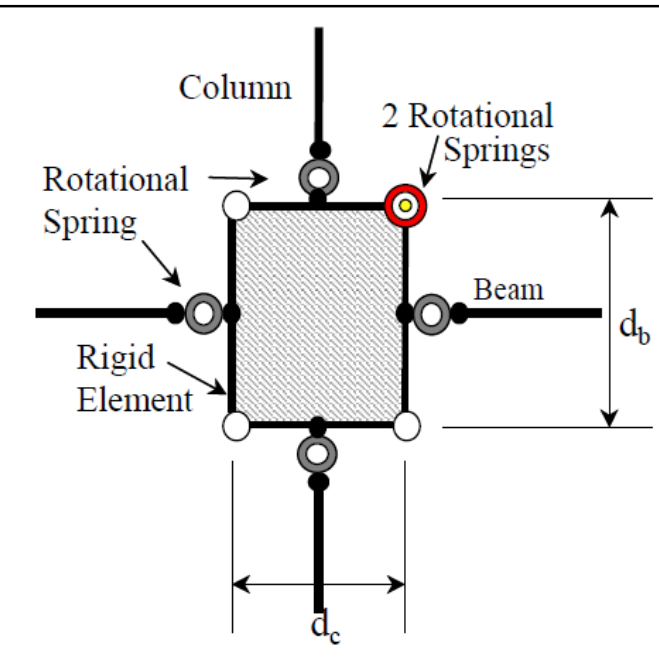

(b)

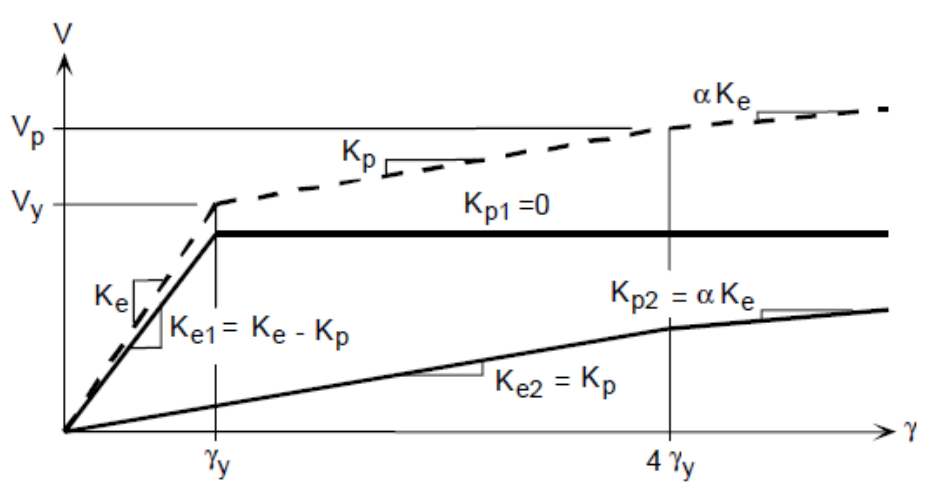

(c)

Figure 2-23. (a) Simplified analytical model of panel zone (scissors model), (b) panel zone model with rigid boundaries (parallelogram model) (c) two springs model simulating the trilinear response (Gupta and Krawinkler, 1999) 


\subsubsection{Modeling of Gravity Connections}

Gravity or shear connections make up a large percentage of the connections in steel momentresisting structures. The gravity connections are usually assumed to be pinned and their contribution to lateral stiffness and strength is conventionally ignored in lateral resisting design and evaluations. However, considering the composite action with the floor slab they may in reality be able to provide a significant contribution to the building's lateral resistance, predominantly in large cyclic deformation when moment frames undergo significant strength and stiffness degradations.

Steel connections (either moment-resisting or shear connections) in concrete slab floor structures are rarely designed as composite systems. Ignoring the composition action results in underestimates of the strength and stiffness of the beams. The composite action also results in shifting in the neutral axis upward along the beam depth which results in a high strain in the bottom beam flange in moment resisting frames. In addition, the slab provides lateral bracing and confinement that tends to prevent beam lateral-torsional buckling and delay development of local buckling in the top flanges. Studies have shown that the typical attachment of the floor slab to girders using shear studs can result in the development of significant composite action, while the reinforcement of the slab and the attachment are typically designed merely for serviceability (Leon, 1990; Leon, Hajjar, \& Shield, 1997).

Several experimental dynamic studies have been conducted to evaluate the seismic response of steel frames with semi-rigid connections with a typical configuration of bolted seat, top and web angles but not including the composite action of the floor slab (Elnashai, Elghazouli, \& DeneshAshtiani, 1998; Nader \& Astaneh-Asl, 1996). The results indicate that semi-rigid frames respond almost as well as full resisting frames in service-level and moderate-level ground motions, although yielding of the seat and top angles led them to experience a large rotation in severe 
ground motions. Respecting their lower stiffness than rigid frames, the semi-rigid frame underwent a lower level of drift compared to a rigid frame with the same capacity to peak ground acceleration ratio (Elnashai, Elghazouli, \& Denesh-Ashtiani, 1998). Elkady and Lignos (2015) showed that considering gravity framing with composite action will result in $50 \%$ increase in shear strength of steel frame buildings with a perimeter SMF system.

Liu and Astaneh-Asl (2000) conducted a comprehensive experimental study including sixteen full-scale tests to evaluate the cyclic response of typical gravity connections. They investigated the effect of composite action due to the floor slab by conducting individual tests on the same gravity frames with and without the floor slab. The obtained results indicated that composite action increases the lateral load capacity of gravity frame to almost twice that of the frame with the bare connection. The typical cyclic response of a gravity frame both with and without floor slab is shown in Figure 2-24. It is shown that after an initial increase in strength and stiffness in frame with slab (Figure 2-24b), at 0.04 radian for the gravity frame configuration, the composite action was terminated by damage to concrete and buckling of metal deck. Afterward, the cyclic response shown is almost identical to the response of the bare connection (Figure 2-24a). The test results indicated that the bare and with slab gravity frames can develop flexural strength up to $20 \%$ and $50 \%$ of the plastic flexural capacity of the beam respectively. 


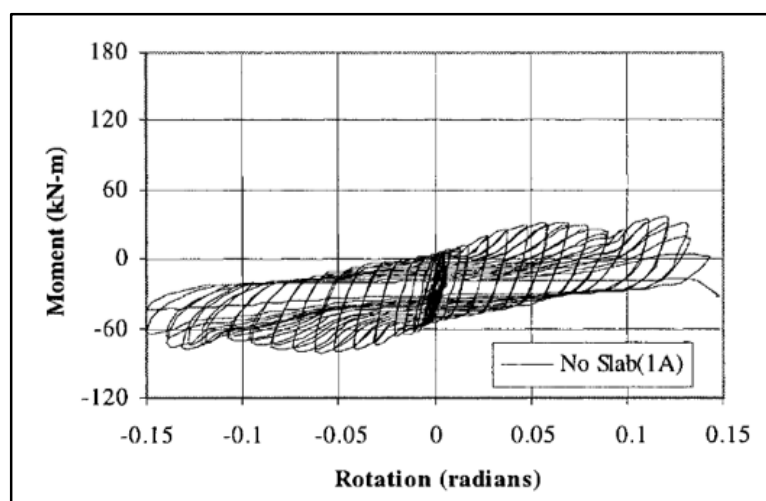

(a)

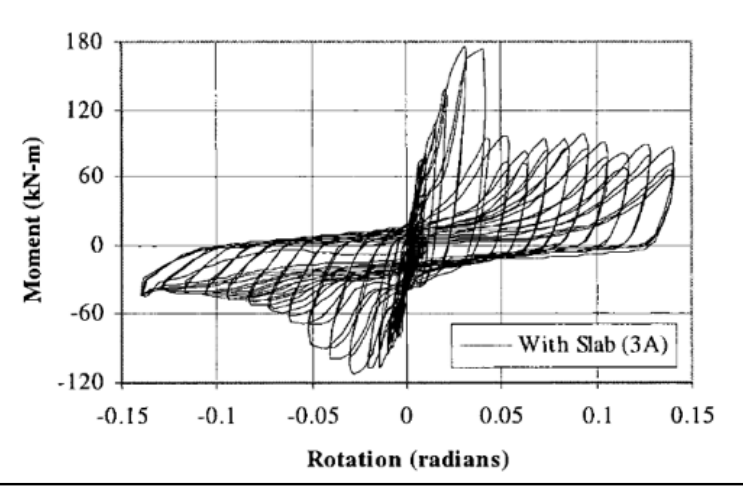

(b)

Figure 2-24. Comparison of moment-rotation response of a gravity framing with and without the floor slab (Liu \& Astaneh-Asl, 2000)

The study showed that the general cyclic response of bare gravity connections is characterized by slip and yielding of shear tabs, followed by yielding of bolt holes and warping in the connection and the end of the beam. Nevertheless, the general cyclic response of the connection and slab is significantly influenced by shifting of the neutral axis which results in strain concentration and consequent local buckling in the bottom flange. It also causes the fracture to be first initiated in the bottom of the connection. The test observations indicated that, even after fracturing of the connection, the gravity frame was able to carry the full gravity loads.

FEMA P-440A (FEMA, 2009) suggests the typical force-displacement backbone response of gravity frames shown in Figure 2-25a. the response includes an almost linear behavior up to maximum strength, and then a strength drop corresponding to loss of composite action, followed by a plateau with a residual strength of $55 \%$ of the maximum strength. The extent of the plateau represents the ductility of the subsystems which depends on the configuration of the gravity connection. When enough rotation in the joint is developed to result in contact between the beam and column, the bolts in shear tabs reach bearing strength failure and the plateau ends. Therefore, the gap between beam and column flange is the major parameter governing the ductility characteristics of the gravity frames. For the beam and column flange, Figure 2-25b, shows the idealized pinched hysteretic response cyclic behavior without cyclic degradation. 


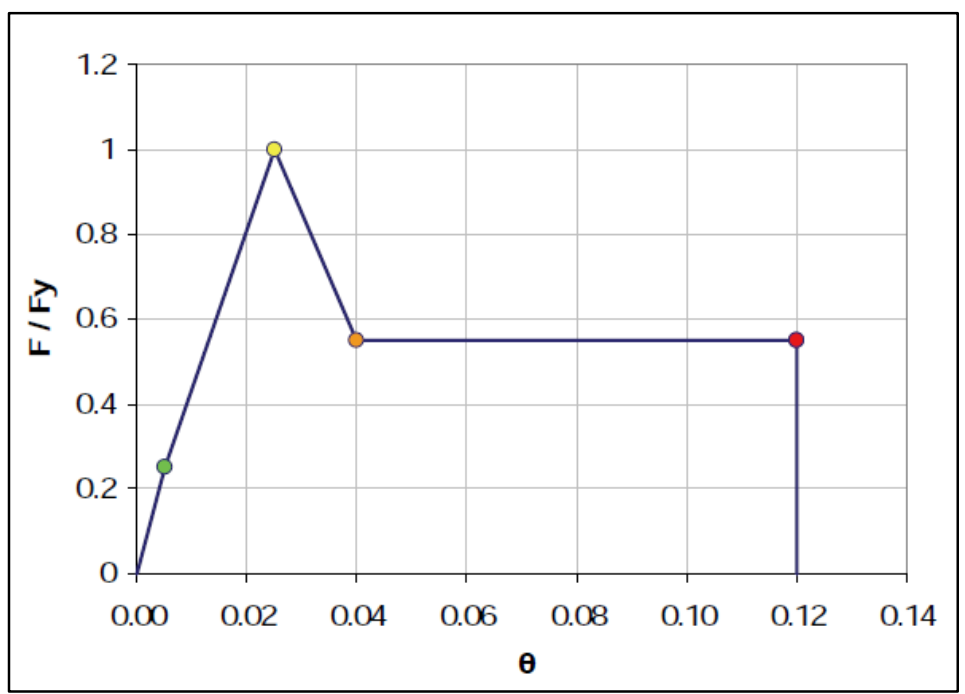

(a)

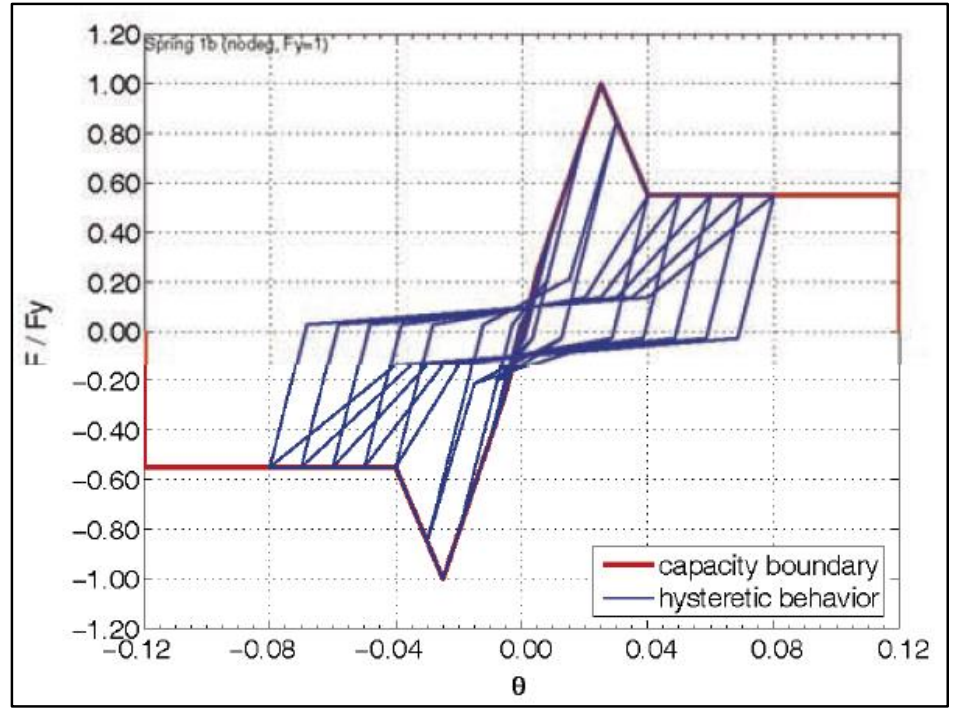

(b)

Figure 2-25. Typical gravity frame response model: (a) the force-displacement capacity backbone; (b) the hysteretic behavior

Elkady and Lignos (2015) developed an approach for explicitly modeling the cyclic behavior of a single-tab gravity connection which was adopted in this study. The approach includes implementing the Pinching4 material model (shown in Figure 2-26a) in OpenSEES to simulate pinched moment-rotation hysteresis behavior of shear tab connections with the floor slab. Pinching4 is able to simulate the pinched force-deformation response of a structural component including cyclic strength and stiffness degradations. As shown in Figure 2-26b, the suggested 
idealized moment-rotation backbone is characterized by: (1) flexural strength and rotation corresponding to bolt slippage $\left(M_{\text {slip }}, \theta_{\text {slip }}\right)$, (2) flexural strength and rotation corresponding to maximum capacity $\left(M_{\operatorname{Max}}, \theta_{\operatorname{Max}}\right)$, (3) flexural strength and rotation corresponding to drop in flexural strength due to concrete slab crushing $\left(M_{\text {drop }}, \theta_{\text {drop }}\right)$, and (4) ultimate rotation capacity, where the connection fails and binding occurs between the steel beam and the column face ( $\theta_{u}$ or $\left.\theta_{\text {blind }}\right)$.

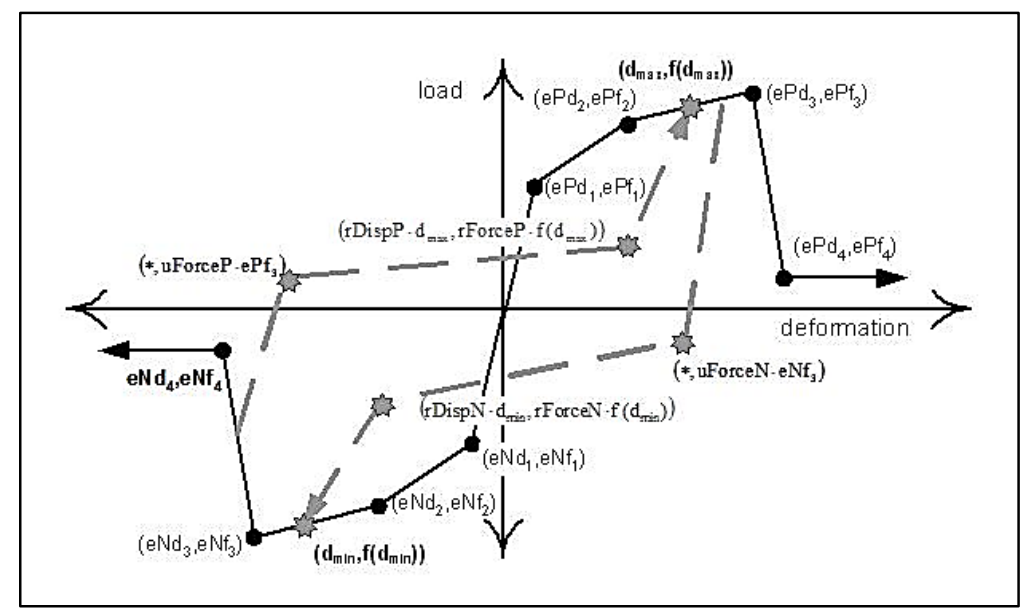

(a)

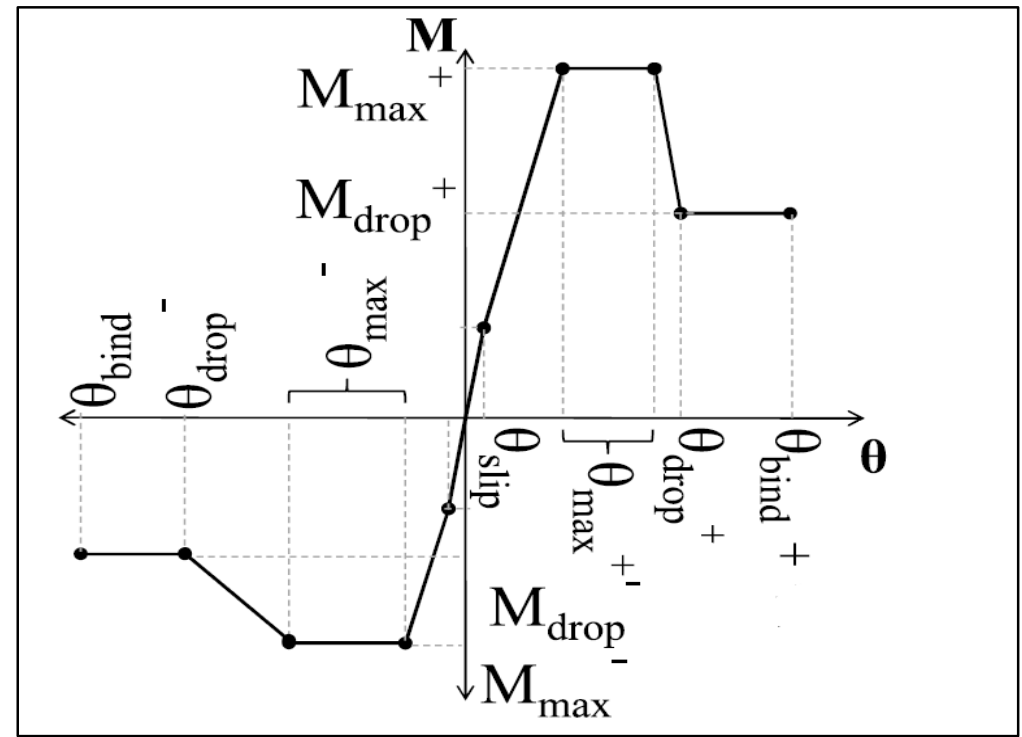

(b)

Figure 2-26. (a) Pinching4 material model (b) Idealized moment-rotation backbone curve simulating the response of composite shear tab connections (Elkady \& Lignos, 2015) 
Elkady and Lignos calibrated the moment-rotation backbone to fit the experimental cyclic response of gravity connections conducted by Liu and Astaneh-Asl (2000). The average parameters for two shear tab connections, connecting $\mathrm{W} 18 \times 35$ and $\mathrm{W} 24 \times 55$ girders to respectively the weak and strong axes of a W14x90 column is shown in Table 2-5. These average parameters were used to develop the gravity connection simulation model in this study.

Table 2-5. The average parameters for developing calibrated moment-rotation backbone of composite shear tab connections (Elkady \& Lignos, 2015)

\begin{tabular}{|c|c|c|c|c|c|c|c|c|c|}
\hline$\frac{M_{\text {Max }}}{M_{p}}$ & $\frac{M_{\text {slip }}}{M_{\text {Max }}{ }^{+}}$ & $\frac{M_{\text {Max }}{ }^{-}}{M_{\text {Max }}{ }^{+}}$ & $\frac{M_{\text {drop }}{ }^{+}}{M_{\text {Max }}{ }^{+}}$ & $\frac{M_{\text {drop }}{ }^{-}}{M_{\text {Max }}{ }^{-}}$ & $\theta_{\text {slip }}$ & $\theta_{\text {Max }}{ }^{+}$ & $\theta_{\text {Max }}^{-}$ & $\theta_{\text {drop }}^{+}$ & $\theta_{\text {drop }}{ }^{-}$ \\
\hline 0.38 & 0.25 & 0.62 & 0.62 & 0.53 & 0.004 & $\begin{array}{c}0.020- \\
0.036\end{array}$ & $\begin{array}{c}0.012- \\
0.028\end{array}$ & 0.040 & 0.060 \\
\hline
\end{tabular}

As shown in Figure 2-26a, in the Pinching4 model cyclic deterioration is characterized by some parameters. The Pair Parameters ( rDispP, rForceP) and ( rDispN, rForceN) define the ratio of demand-rotation and moment of the previous loading cycle at which reloading begins in the positive and negative loading direction respectively. Parameters $u F$ orceP and $u$ ForceN define the ratio of strength developed upon unloading from positive and negative load to the maximum strength developed under monotonic loading. Three parameters gKLim, gDLim, and gFLim, restrict the cyclic degradation corresponding to loading stiffness, unloading stiffness and flexural strength respectively. Table 2-6 represents the average parameters developed by Elkady and Lignos to match experimental cyclic response of gravity connections conducted by Liu and Astaneh-Asl (2000). These average parameters were used to develop the gravity connection simulation model in this study. 
Table 2-6. The average parameters for developing pinching and degradation composite shear tab connections cyclic response (Elkady \& Lignos, 2015)

\begin{tabular}{|c|c|c|c|c|c|c|c|c|}
\hline rDispP & rForceP & uForceP & rDispN & rForceN & uForceN & gKLim & gDLim & gFLim \\
\hline 0.40 & 0.22 & 0.07 & 0.50 & 0.42 & 0.02 & 0.30 & 0.07 & 0.05 \\
\hline
\end{tabular}

For the studied buildings, the type of gravity connection is not specified in the available documents but based on typical practice in the construction industry, conventional bolted single shear tab connections were assumed. Typical floor beams of W14x80 were considered for all three buildings. Therefore, by implementing the pinching 4 model and using parameters presented in Table 2-5 and Table 2-6 for W14x80 gravity beams, the moment-rotation model for the gravity connections was obtained as shown in Figure 2-27. The ultimate rotational capacity of the gravity connection was assumed to be equal to $\theta_{\text {blind }}=0.15$. By considering the limited rotational capacity of the connection which might govern in collapse prediction, this assumption is probably not be critical.

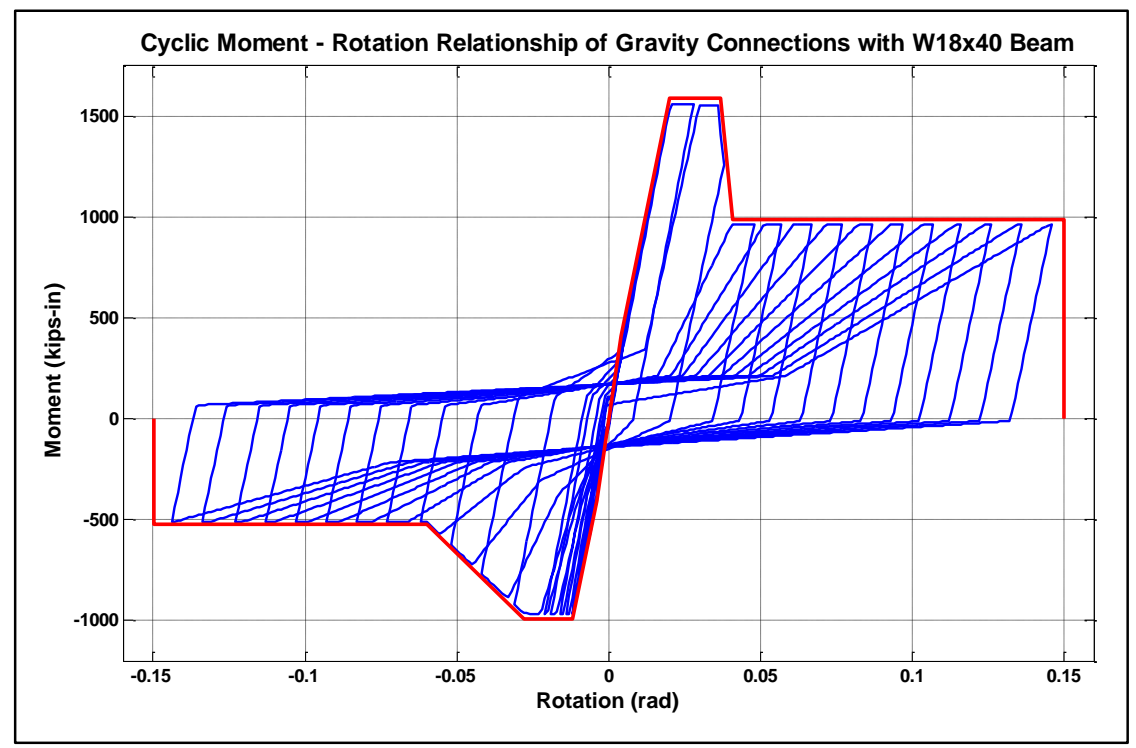

Figure 2-27. Moment-rotation backbone and enclosed cyclic response of the 47-story building's gravity connections with W18x40 beams 


\subsubsection{Gravity Loads and Inertial Masses}

Nonlinear analysis, unlike linear analysis, is load path dependent, therefore a combination of all type of expected load needs to be considered to ensure that a realistic response of the structure is obtained. Accordingly, in this study, a combination of gravity and the wind lateral loads was simultaneously applied to the structural model. According to ASCE 7-10-13 the gravity loads, $Q_{G}$, used in nonlinear seismic evaluation approaches should be according to following combination:

$$
Q_{G}=Q_{D}+Q_{L}+Q_{S}
$$

where $Q_{D}, Q_{D}$ and $Q_{D}$ are the dead load, live load and snow load respectively acting on the structure.

The expected gravity load generally is a combination of unfactored dead load and a fraction of design live load (or snow load for roof level, whichever is greater). Generally, the expected dead load includes: the structure self-weight, architectural finishes (partitions, exterior wall, floor and ceiling finishes) and dead loads associated with utility services and equipment. The expected live load generally is considered as a fraction of nominal design live load. The reduction in live load is characterized by: (1) low probability of occurrence of nominal live load all over the building and (2) low probability of co-occurrence of the nominal live load and extreme wind load (PEER/ATC, 2010).

The expected gravity loads for all buildings were mentioned earlier. The entire gravity load was included in the numerical model in order to capture geometry nonlinearity associated with the Pdelta effect. The typical 2D nonlinear analysis considers leaning columns with applied gravity loads to properly simulate the P-delta effect. In this study all gravity framings were included in a 3D numerical model including gravity framing, therefore, the P-delta effect was explicitly included. 
The inertial mass considered in the numerical model included the self-weight of the building plus some allowance for contents. The masses were lumped at all beam-column junctions (both moment frame and gravity frames) at the floor levels to accurately include inertial effects in the two horizontal directions, as well as rotation about the vertical building axis.

\subsubsection{Geometric Nonlinearity}

Gravity loads acting on the deformed structure lead to an increase of internal forces in structural components, which causes geometric nonlinear effects in the structure, known as P- $\Delta$ effects. The P- $\Delta$ geometric nonlinear effects can be caused by two type of deformations: 1) Along- member deformation, measured relative to the chord connecting two ends of the member and 2) Lateral deformation measured between the two ends of the member associated with the story drift. Lateral deformation is a major concern and the along- member deformation is not generally modeled in seismic and wind analyses and was not considered in this study. The P- $\Delta$ geometric nonlinear effects associated with lateral deformation increase the internal forces and, as a result, less capacity remains available to undergo larger lateral loads. It may consequently lead to loss of lateral resistance, larger lateral residual deformations and even dynamic inelasticity (FEMA, 2009).

In the dynamic response, when the response is quite large, corresponding to negative tangent stiffness, the P- $\Delta$ effect can lead to remarkable amplification in displacement response, such that for a response with $5 \%$ negative stiffness of an SDOF system the P- $\Delta$ effect can cause the system to collapse (PEER/ATC, 2010). However, the P- $\Delta$ effect is normally under control in the positive tangent stiffness range.

Figure 2-28 shows pushover curves of the 47-story building in the X-direction using the ASCE 710 wind loading pattern developed with and without considering the P- $\Delta$ geometric nonlinear effects. Significant stiffness reduction is shown in building response due to the P- $\Delta$ geometric 
nonlinear effects which resulted in the loss of lateral resistance and consequently early instability compared to the response without P- $\Delta$ effects. It indicates that the entire gravity loads should be properly included in the numerical model and the P- $\Delta$ geometric nonlinear effect also needs to be directly considered in both static and dynamic analyses.

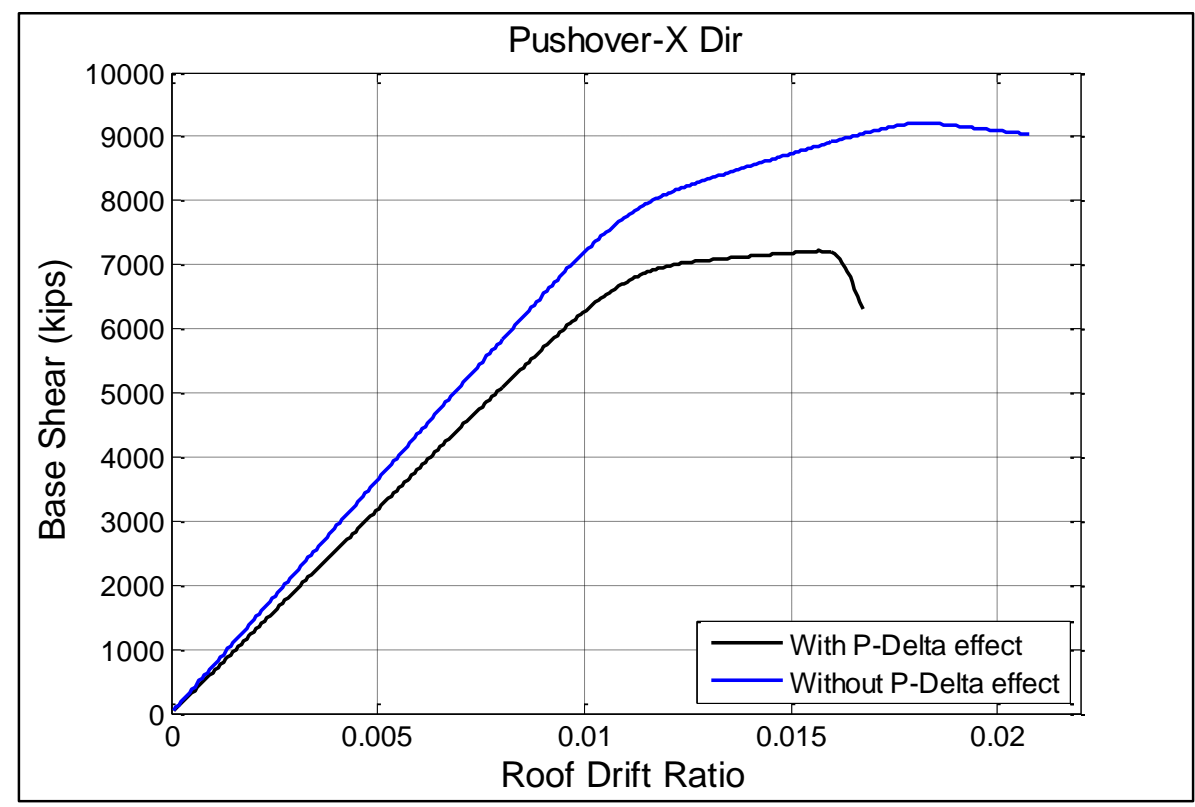

Figure 2-28. Pushover curve responses of the 47-story building using ASCE 7-10 wind load pattern, with and without gravity load illustrating the P-A geometric nonlinear effect

\subsubsection{Shear Deformation of Beams and Columns}

In conventional structural analysis approach, the shear deformations of steel beams and columns are assumed to be insignificant and are neglected. But, it might be significant for high-rise building with a large number of elements where the cumulative shear deformation effect might be significant. Also, for buildings such as the 47-story building with relatively deep beams, neglecting the effect of shear deformations may result in significant errors in lateral stiffness and capacity.

In this study, the effect of shear deformation on the lateral response of the 47-story building was evaluated by conducting pushover analysis using the ASCE 7-10 wind loading pattern for two 
cases: including and neglecting the shear deformation in beams and columns. Figure 2-29 shows the pushover curves for the two cases. It is shown that neglecting shear deformations of the steel beams and columns result in a significant overestimation of the building's lateral stiffness. However, the lateral resistance changed very little.

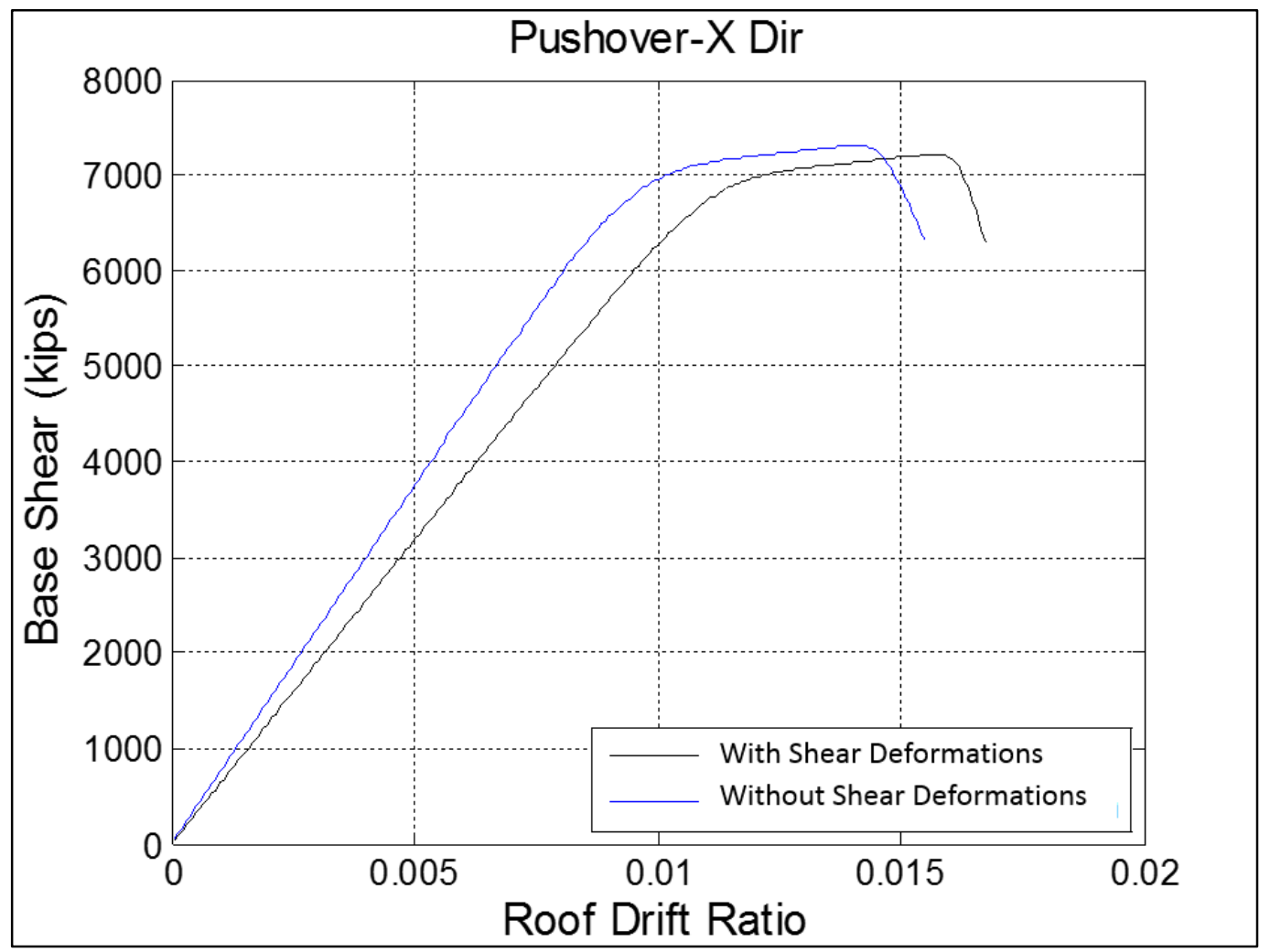

Figure 2-29. Pushover curve responses of the 47-story building, using ASCE 7-10 wind load pattern, with and without beams and columns shear deformations.

\subsubsection{Summary of The Modeling Approach}

In this study, 3D nonlinear analytical models of the three high-rise buildings were developed using OpenSEES version 2.4.6 (PEER, 2015) finite element software. Moment-resisting framing, as well as the gravity framing, were included in the nonlinear structural model. The models consisted of combinations of elastic line elements and nonlinear springs to incorporate the nonlinearity in beams, columns, panel zones and connections. Shear deformation of elements and the P- $\Delta$ geometric nonlinear effect were explicitly considered in the analytical model. Nodal 
constraints were implemented to simulate rigid diaphragm behavior provided by floor slabs in all stories. The analytical modeling approach implemented in this study is summarized by illustrating the modeling details for moment-resisting and gravity framing in Figure 2-30 and Figure 2-31 respectively.

As shown in Figure 2-30, in the moment resisting frames panel zones were modeled with an assembly of rigid line elements and nonlinear springs. The 3D model was developed by adopting parallelogram model proposed by Gupta \& Krawinkler (2000). Nonlinear steel beam and column elements were modeled as assemblies of an elastic linear element and two zero-length nonlinear springs at the ends simulating the development of concentrated inelasticity in the beam/column elements. The cyclic moment-rotation relationship of beams and columns was based on the Modified Ibarra- Krawinkler (MIK) model. As shown in the figure, for the 47-story building, the pre-Northridge connections were simulated by developing a hysteretic moment-rotation model based on available experimental studies and recommendations provided by FEMA 355D. For the 30- and 40-story buildings, with no specified connection details, rather than a cyclic momentrotation relationship, only a rotational limitation equal to 0.02 was implied.

As shown in Figure 2-30, the gravity framing was also incorporated by modeling the columns with lump plasticity as described for the moment resisting frame. The beams were modeled with elastic elements and the semi-rigid gravity connections were explicitly modeled. The treatment of the nonlinear response of typical shear tab gravity connections, including the floor slab composite action effect, made use of the numerical model suggested by Elkady and Lignos based on average parameters obtained from full-scale experimental studies. 


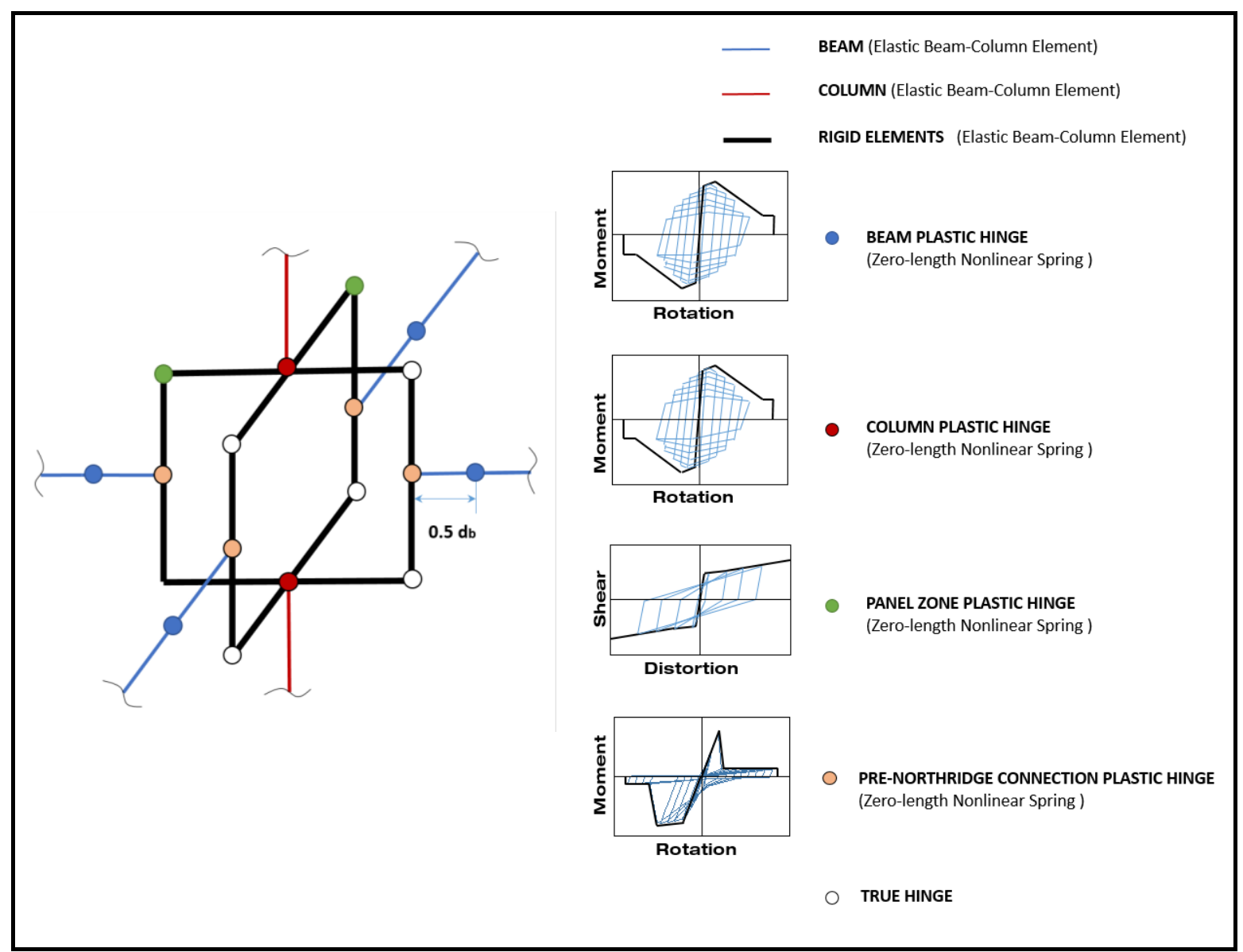

Figure 2-30. Representation of inelasticity modeling in moment-resisting frames

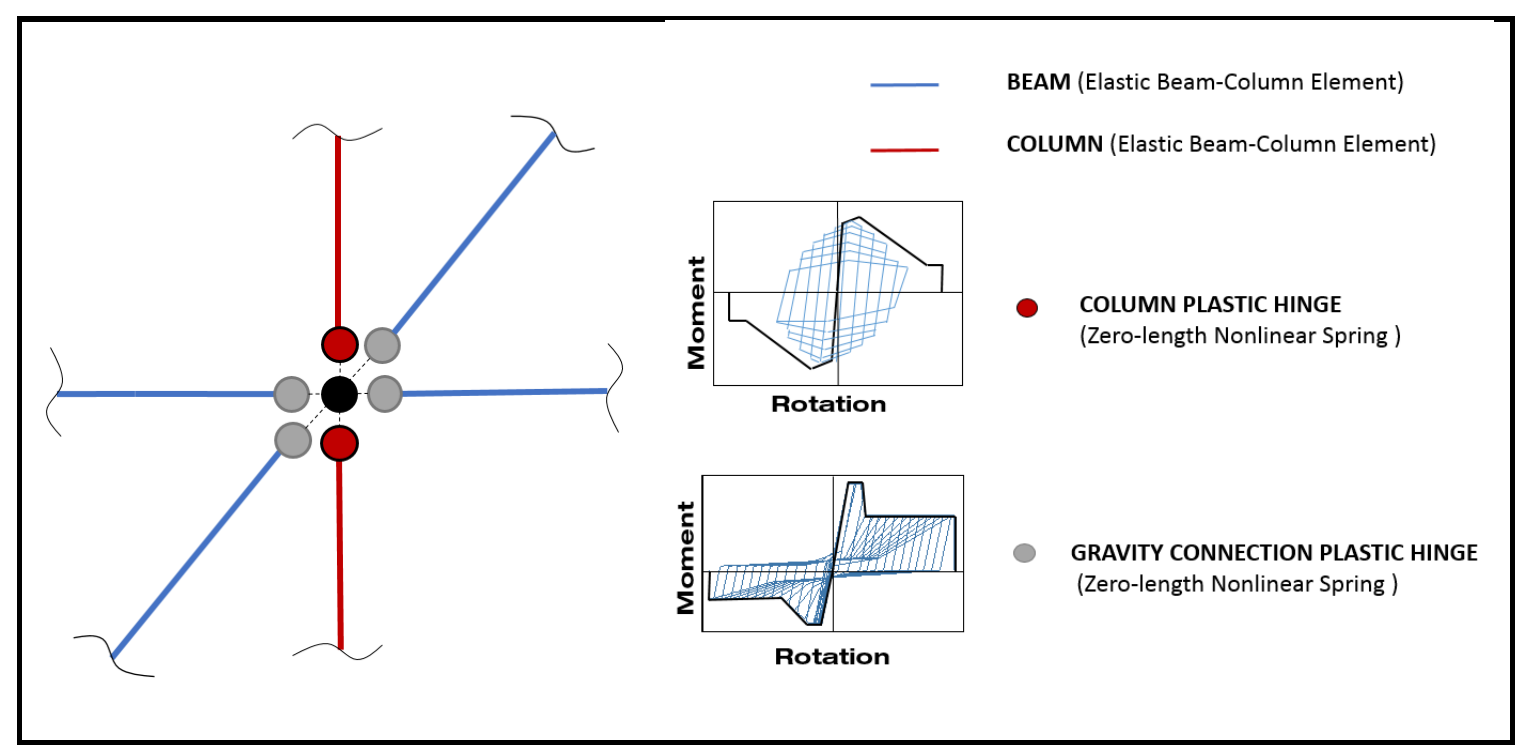

Figure 2-31. Representation of inelasticity modeling in gravity frames 


\section{CHAPTER III}

\section{VALIDATION OF THE ANALYTICAL APPROACH}

\subsection{Introduction}

A reliable analytical approach requires sets of validations which are typically performed by conducting sorts of complementary experimental studies verifying the obtained analytical results. Despite the numerous scaled and full size nonlinear seismic experiments on buildings carried out in the US and around the world, conducting nonlinear wind dynamic experiments has not been a concern in the wind engineering research field. That might be due to the fact that structural component nonlinearity in wind design has not been allowed by current codes and standards. Aeroelastic wind tunnel testing of scaled high-rise models is a practice to acquire wind loads for designing of new high-rise buildings. In this approach, an equivalent aerodynamic model typically representing the first three modes (two fundamental sway and the torsional modes) is subjected to simulated wind boundary layer flow and wind induced forces and acceleration responses are measured. Mooneghi et. al (2015) attempted to develop a bilinear aeroelastic model for tall buildings by incorporating primitive structural nonlinearity to study the effect of softening and hysteretic damping associated with the component nonlinearity in the wind-induced response of high-rise buildings. Despite the rough pioneer attempt, it is yet far from being comparable with seismic nonlinear accurate shake table testing.

Given the above facts regarding the absence of wind nonlinear dynamic experimental data, the analytical approach used in this study was validated by simulating reliable analytical and experimental data available in the literature. This chapter presents the validation analyses conducted in the different stages of this research. 


\subsection{Validation of Fiber-Section Nonlinear Model}

At the early stage of this research, an attempt was made to consider structural component nonlinearity by using the fiber-section method. Despite its advantages in considering the spread of plasticity and capturing the P-M interaction, the major disadvantage was in its inability to capture the cyclic deteriorations, due to its use of the concentrated plasticity of the MIK model. While the fiber-section was not the final approach, the performed validation study which involved comparing three different nonlinearity modeling methods as well as a hand calculation check is presented below.

A 3D 2-story steel structure (as shown in Figure 3-1) modeled in OpenSEES, ANSYS, and SAP2000. The model consists of steel hollow tube column and I-shape beam as illustrated in Figure 3-2. The beams are subjected to uniformly distributed dead and live load as $W_{d l}=1160 p l f$ and $W_{l l}=285 p l f$ respectively. 


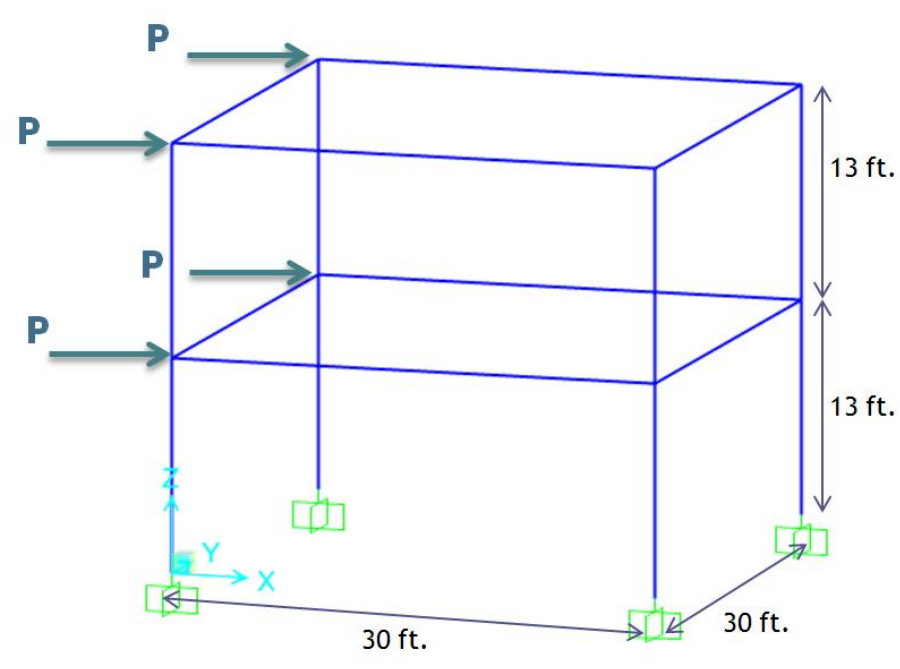

Beam Section:

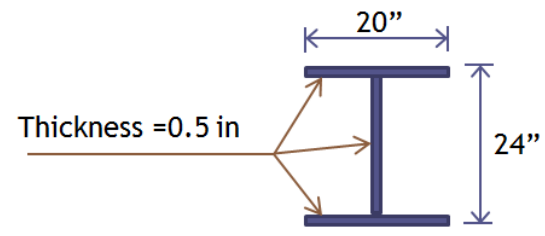

Column Section:

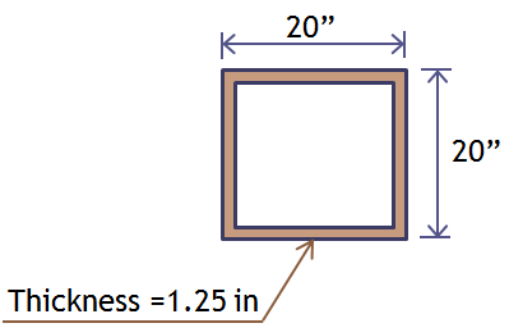

Figure 3-1. Details of the single-bay 2-story model.

The structure was modeled in OpenSEES using nonlinear beam-column element and fiber section discretization. Figure 3-2 shows the typical fiber section subdividing used in this study. Five integration points along each member were considered for all beam and column elements. As shown in the picture the I-shape members (either beams or columns) are divided into 160 fibers including $64(16 \times 4)$ fibers in flanges and $32(16 \times 2)$ fibers in the web. The box section columns are divided to 256 fibers including $64(16 \times 4)$ fiber in each wall.

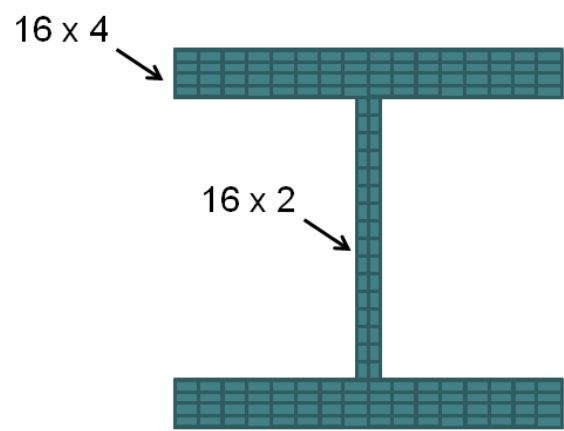

$16 \times 4$

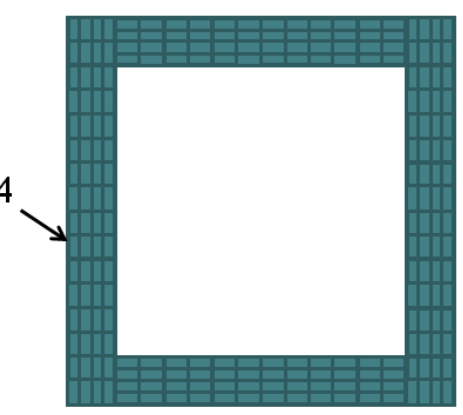

Figure 3-2. Typical fiber-section subdividing for the I-shape and box steel members. 
Force-based elements were used in the modeling. While displacement based analysis is commonly used to get the structural response, some studies have highlighted the benefits of the analysis based on force interpolation functions. By using force interpolation functions (flexibilitybased method), the solution involves only numerical integration errors. The errors can be reduced by increasing the number of the integration points along the length of the element. However, by using deformation-based elements, only refinement of the finite element meshing and consequently higher computational effort can improve the solution (Neuenhofer \& Filippou, 1997).

The Giuffré-Menegotto-Pinto constitutive model is used as the constitution of steel in this study. The model suggested by Giuffré and Pinto (Giuffrè \& Pinto, 1970) and later developed by Menegotto and Pinto (Menegotto \& Pinto, 1973). Figure 3-3 shows both typical monotonic and cyclic behavior of the model. The model contains the isotropic hardening in the cyclic behavior. The model well matched the result obtained from cyclic loading test of steel. 


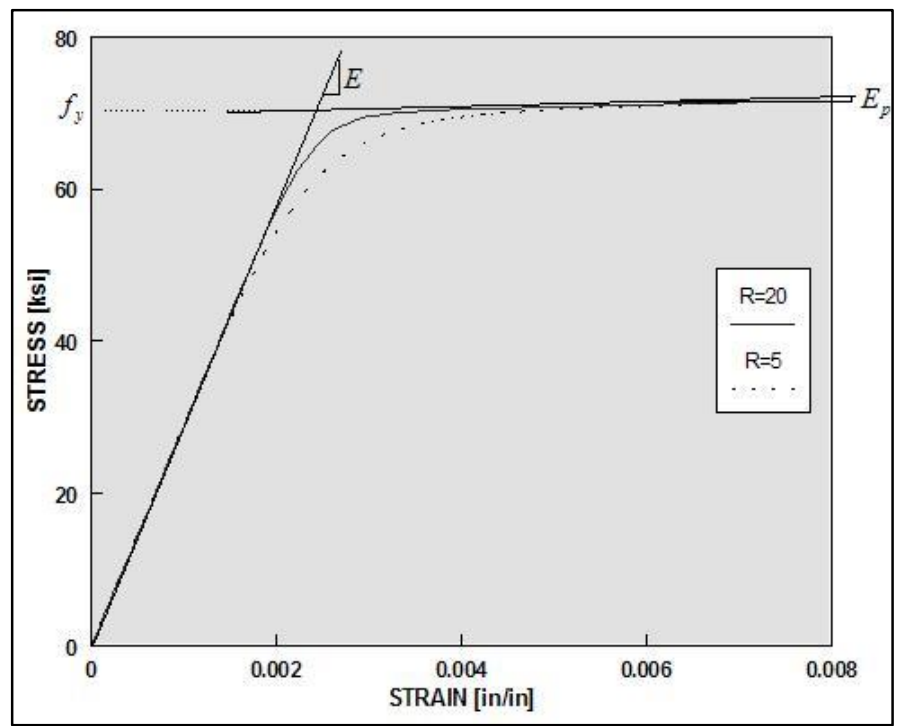

(a)

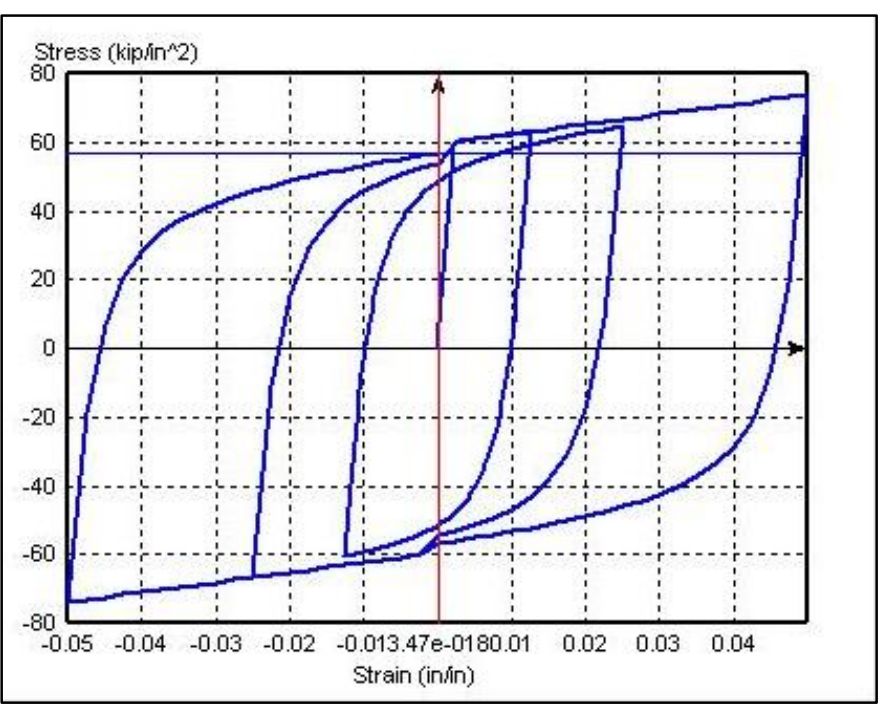

(b)

Figure 3-3. Giuffré-Menegotto-Pinto constitutive model for steel: (a) typical monotonic behavior (b) typical cyclic behavior with isotropic hardening

In the ANSYS model, the elements were modeled using SHELL181 and the nonlinearity was provided by assigning nonlinear steel model to shell elements. Finally, in SAP2000, beams and columns were modeled with linear beam-column elements and the nonlinearity was added through considering zero-length concentrated hinges in both ends of all beams and columns. The mass related to each story was added to the model by considering lumped mass at four beam- 
column junctions in each story level. No additional constraints such rigid diaphragm were considered in the models.

After applying the gravity load, the structure was pushed with four increasing point loads as shown in Figure 3-1. The Roof Displacement versus Base shear diagrams obtained from three different models are shown in Figure 3-4. The figure indicates that OPENSSES and ANSYS models are matching well, while the SAP2000 resulted in a slight higher lateral capacity.

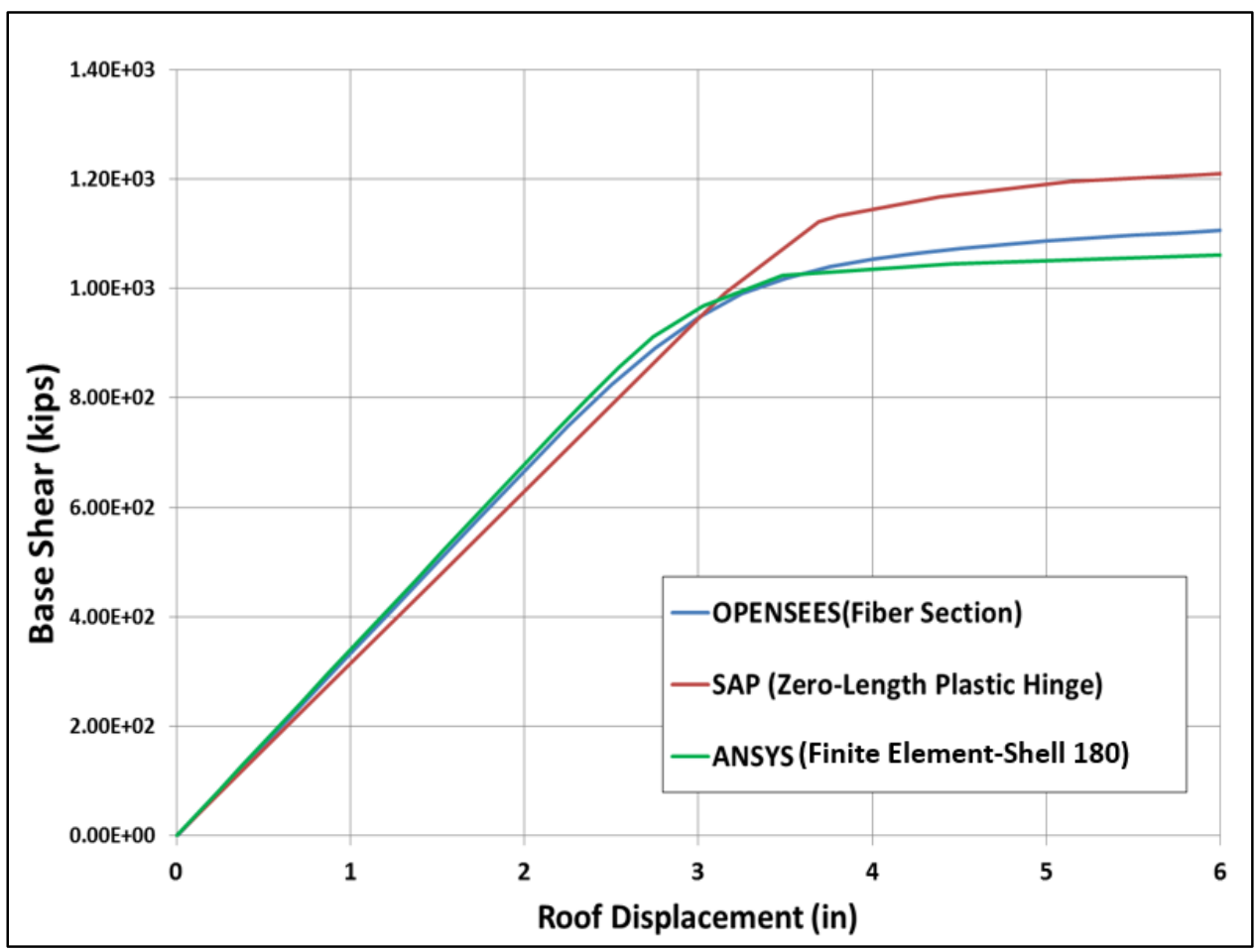

Figure 3-4. Pushover analysis results of the three models to compare the accuracy in simulating the nonlinearity.

Figure 3-5 shows the deformed shape and contours of stresses in the push direction obtained from ANSYS. The deformed shape indicated elastic compression flange local buckling as the failure mode. 

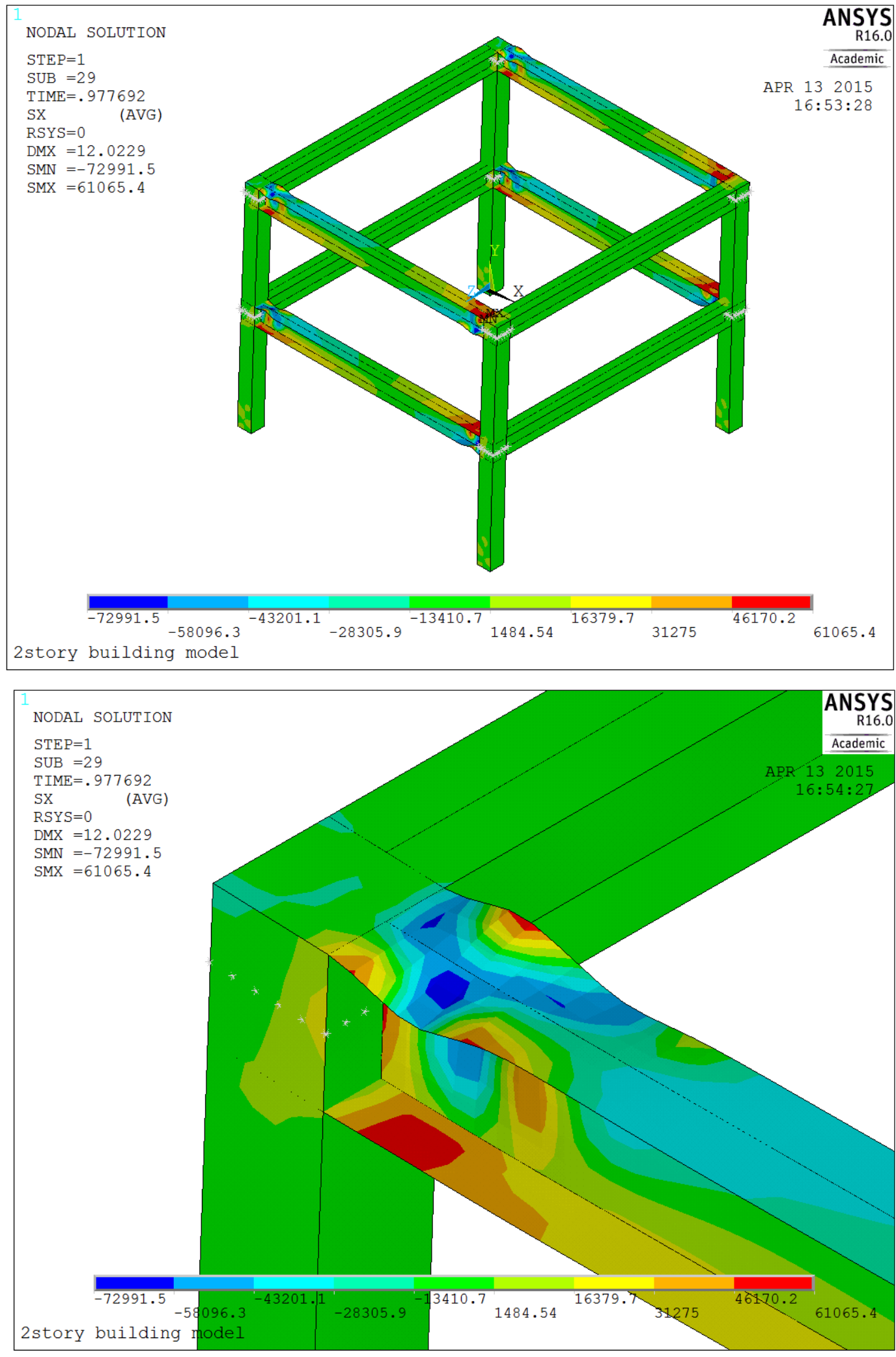

Figure 3-5. Deformed shape and along-push direction stress contours of the 2-story frame 
The same failure mode is concluded by checking through AISC LRFD (AISC-360, 2005) as shown below:

- $\quad$ Flange Slenderness

$\left\{\begin{array}{l}\frac{b}{t}=\frac{10}{0.5}=20 \\ \lambda_{p}=0.38 \sqrt{E / f_{y}}=9.12 \quad==>\quad \text { Non }- \text { compact Flange } \\ \lambda_{r}=1.0 \sqrt{E / f_{y}}=24\end{array}\right.$

- Web Slenderness

$\left\{\begin{array}{l}\frac{h}{t_{w}}=\frac{22.5}{0.5}=45 \\ \lambda_{p}=3.76 \sqrt{E / f_{y}}=90.24 \quad==>\quad \text { Compact } W e b \\ \lambda_{r}=5.7 \sqrt{E / f_{y}}=136.0\end{array}\right.$

- Non-compact flange and compact web $\rightarrow$ possible failure modes: compression flange buckling and lateral torsional buckling.

1. Lateral buckling

$$
\begin{gathered}
\left\{\begin{array}{c}
L_{b}=340 \mathrm{in} \\
L_{p}=1.76 r_{y} \sqrt{E / f_{y}}=194.3 \\
L_{r}=1.95 r_{t s} \frac{E}{0.7 \times f_{y}} \sqrt{\frac{J c}{S_{x} h_{0}}} \sqrt{1+\sqrt{1+6.76\left(\frac{0.7 f_{y}}{E} \frac{S_{x} h_{0}}{J c}\right)^{2}}}=414 \mathrm{in}
\end{array}\right. \\
\quad \rightarrow \quad M_{n}=C_{b}\left[M_{p}-\left(M_{p}-0.7 f_{y} \times S_{x}\right)\left(\frac{L_{p}-L_{b}}{L_{p}-L_{r}}\right)\right] \\
=21241 \text { kips }- \text { in }>M_{p}=15068
\end{gathered}
$$

2. Compression Flange Buckling

$$
M_{n}=\left[M_{p}-\left(M_{p}-0.7 f_{y} \times S_{x}\right)\left(\frac{\lambda_{p}-\lambda_{b}}{\lambda_{p}-\lambda_{r}}\right)\right]=11026 k i p s-\text { in }<M_{p}=15068
$$


As described above pushover analyses were conducted using three different nonlinearity modeling methods including; concentrated hinges, fiber-section model, and shell finite element modeling method. From the obtained results, it can be concluded that all three models showed good agreement in nonlinear static loading, although ANSYS (shell finite modeling) and OpenSEES (Fiber-section model) showed more comparable results than SAP2000 (concentrated hinges) models with slightly higher lateral capacity. The hand calculation using the AISC LRFD (AISC-360, 2005) method indicated the failure mode is consistent with ANSYS finite element model results.

\subsection{Validation of Concentrated Hinge Model}

By enhancing the analytical approach though changing from a fiber-section modeling method to a concentrated hinge model, a verifying analysis to ensure the accurate implementation of the new method was required. To validate the approach, nonlinear pushover analysis of a 2D 2-story SMF frame presented by NIST GCR 10-917-8 report (NIST, 2011) also used by Flores et. al (2014) was repeated.

The three-bay 2-story frame model investigated in this study represents the lateral resisting system of an SMF building using Reduced Beam Section (RBS) connections. The building plan is shown in Figure 3-6a. The frame consisted of elastic beam/column elements and concentrated plastic hinges at ends and parallelograms model representing panel zone nonlinear behavior as described in the previous chapter. Beam and column plastic hinges were modeled using monotonic backbone and cyclic response rules based on the Modified Ibarra-Kraweinler model. The Panel zone model was a model with a trilinear backbone curve and no cyclic deterioration as all described in previous chapter. 


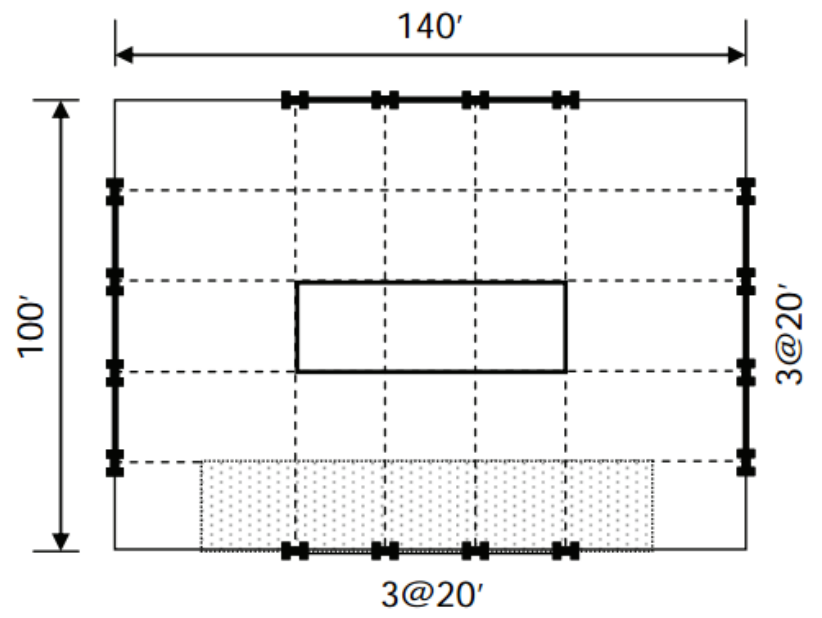

(a)

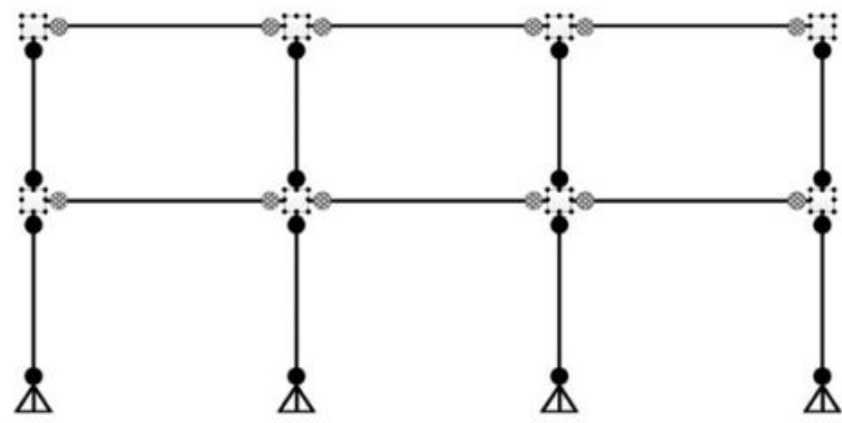

- Plastic Hinges Beams (RBS)

- Plastic Hinges Columns

::: Panel Zones

(b)

Figure 3-6. 2-story steel SMF: (a) the representative building plan;(b) modeling details

P-delta effect was modeled with a leaning column loaded with half gravity load (1.05D+0.25L) of each floor acting on gravity columns which were not included in the idealized model. Nonlinear static pushover analysis was performed using the loading pattern according to fundamental mode shape of the frame. The pushover curve obtained in this study is compared to results presented by Flores et. al (2014) which are identical to results reported in the NIST report. The comparison shows a good agreement between the obtained pushover results and available results which indicates the validation of analytical implemented approach. 


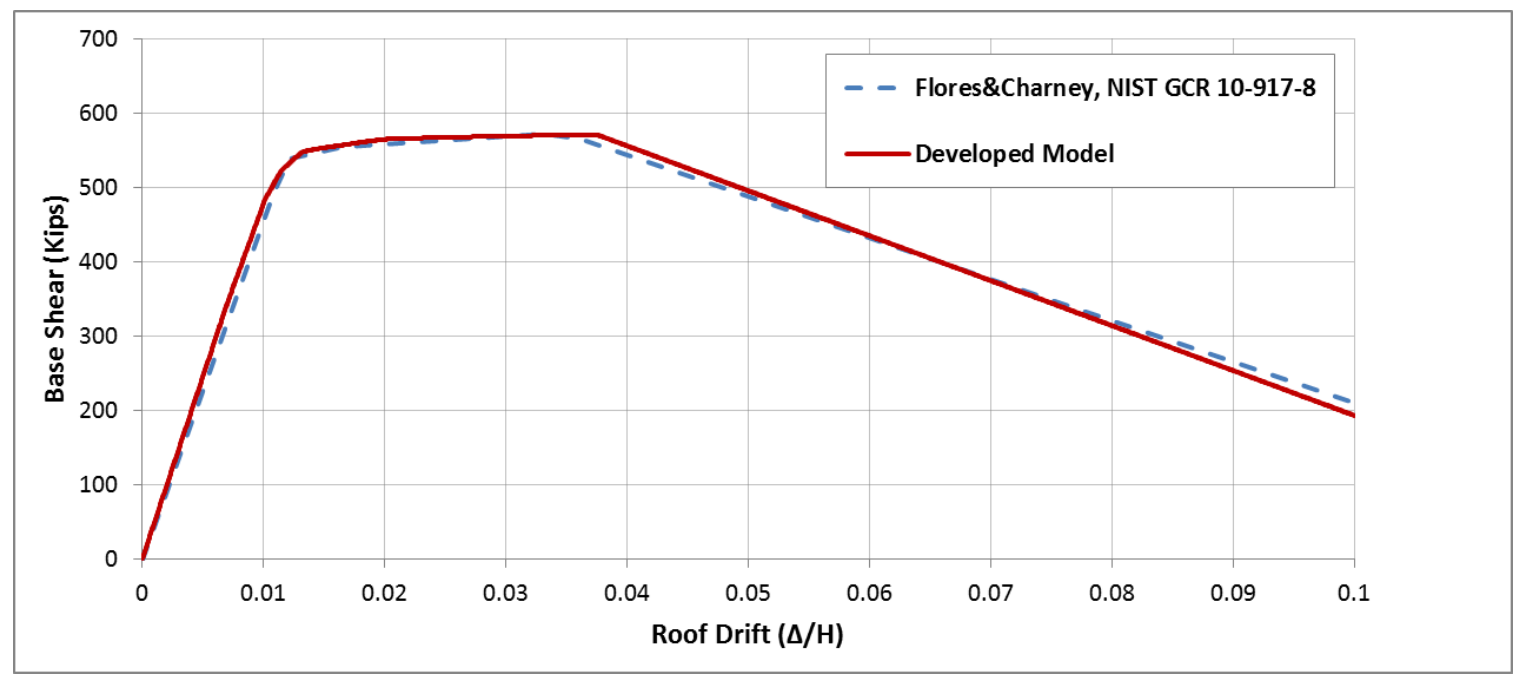

Figure 3-7. Comparison between obtained pushover curves of the 2-story frame

\subsection{Validation of The Modeling of Semi-Rigid Connections}

As discussed in the previous chapter, gravity framing can contribute to structure's lateral stiffness and strength significantly. While the typical gravity connections are considered pinned in building design, experimental studies have shown they are able to develop semi-rigidity affecting the structures lateral response, particularly when they benefit from composite action provided by proper attachment to floor slab. In this study, to achieve a more realistic response of a high-rise building the gravity framing was incorporated into the analytical models considering the full nonlinear moment-rotation response of semi-rigid gravity connections.

In the early stage of this research to validate the approach used to model the nonlinear response of gravity connections, an investigation was conducted. In this investigation, the study on the response of a 2D 3-story frame with different types of semi-rigid connection as presented by Azizinamini (1985), was repeated to validate the approach by comparing the results. The example 2D 3-story frame, as well as the framing information and lateral loading are shown in Figure 3-8a. The considered semi-rigid connections include four top-and-seat and web angle connection and two top-and-seat connections. The details of the connections are shown in Figure 3-8b and 3-8c. The nonlinear moment-rotation response for all semi-rigid connections are presented by 
Azizinamini as shown in Figure 3-9. Among the six mentioned connection cases, four cases are investigated in this study in addition to two pinned and fixed connection cases.

In Azizinamin's approach the nonlinear semi-rigidity of the connection was incorporated in the stiffness matrix of the beam and then the frame was analyzed under given lateral loads to estimate the lateral response for each case. In the approach implemented in this study the frame was modeled with elastic beams and columns and the nonlinear moment-rotation relationship for semi-rigid connections were modeled using zero-length rotational springs. The nonlinear response was introduced using OpenSEES Multilinear Uniaxial Material.

The story displacement response obtained from both OpenSEES analytical model and the results presented by Azizinamini are shown in Table 3-1. The table presents story displacement at all three stories for all six cases including: fixed connections, pinned connections, two top-and-seat and web angle connections (case1 and 4) and two top-and-seat connection types (case5 and 6). Comparing the obtained results indicates a good agreement between two methods and it verifies the approach implemented in this study. 


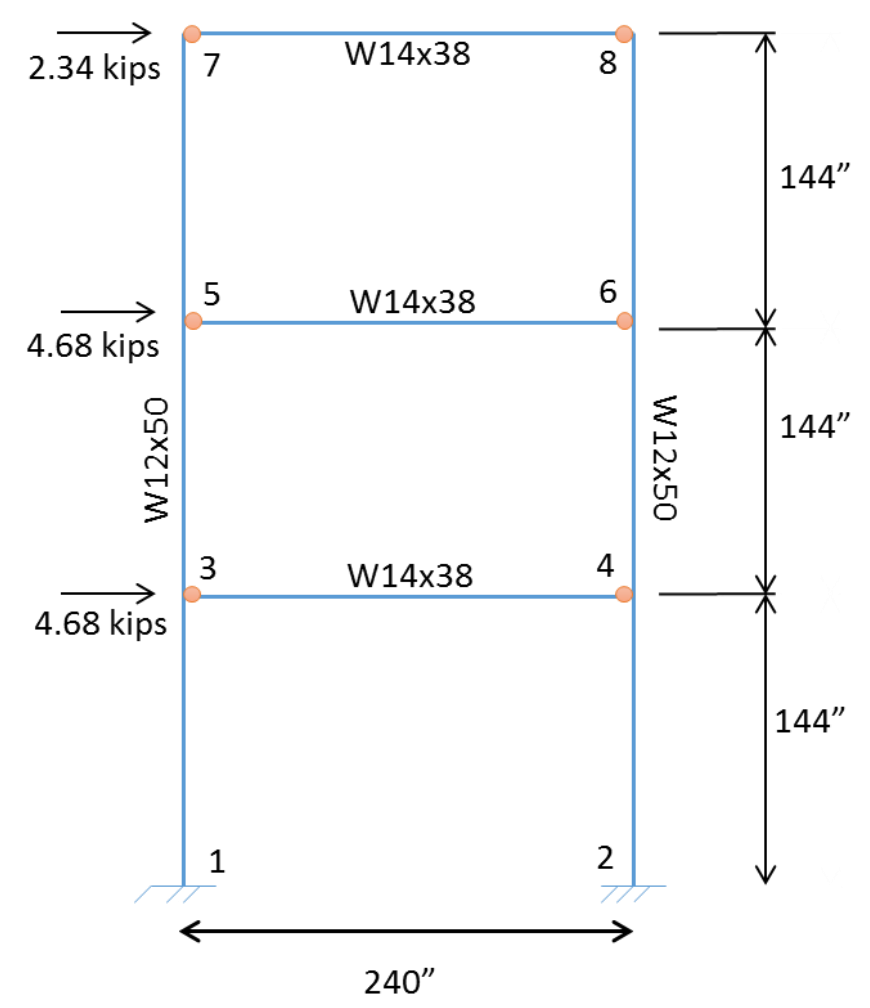

(a)

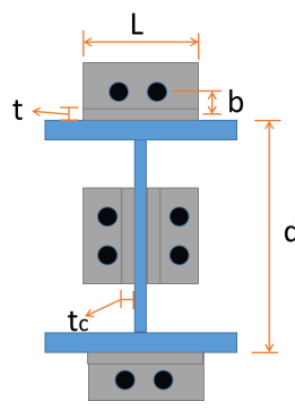

\begin{tabular}{|l|l|l|l|l|l|}
\hline Case & $\begin{array}{l}\text { T } \\
\text { inches }\end{array}$ & $\begin{array}{l}\text { d } \\
\text { inches }\end{array}$ & $\begin{array}{l}\text { tc } \\
\text { inches }\end{array}$ & $\begin{array}{l}\text { L } \\
\text { inches }\end{array}$ & $\begin{array}{l}\text { b+t/2 } \\
\text { inches }\end{array}$ \\
\hline d Case \#1 & 0.375 & 14.1 & 0.25 & 8 & 2.125 \\
\hline Case \#2 & 0.5 & 14.1 & 0.25 & 8 & 2.125 \\
\hline Case \#3 & 0.375 & 14.1 & 0.375 & 8 & 2.125 \\
\hline Case \#4 & 0.5 & 14.1 & 0.375 & 8 & 2.125 \\
\hline
\end{tabular}

(b)

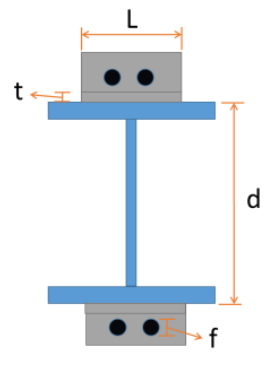

\begin{tabular}{|l|l|l|l|l|}
\hline & $\begin{array}{l}\text { T } \\
\text { inches }\end{array}$ & $\begin{array}{l}\text { d } \\
\text { inches }\end{array}$ & $\begin{array}{l}\text { tc } \\
\text { inches }\end{array}$ & $\begin{array}{l}\text { L } \\
\text { inches }\end{array}$ \\
\hline Case \#5 & 0.375 & 14.1 & 0.75 & 8 \\
\hline Case \#6 & 0.5 & 14.1 & 0.75 & 8 \\
\hline
\end{tabular}

(c)

Figure 3-8. Sample analysis on semi-rigid connections: (a) sample plan frame; (b) details of the top-and-seat and web angle type connections; (c) details of the top-and-seat type connections 


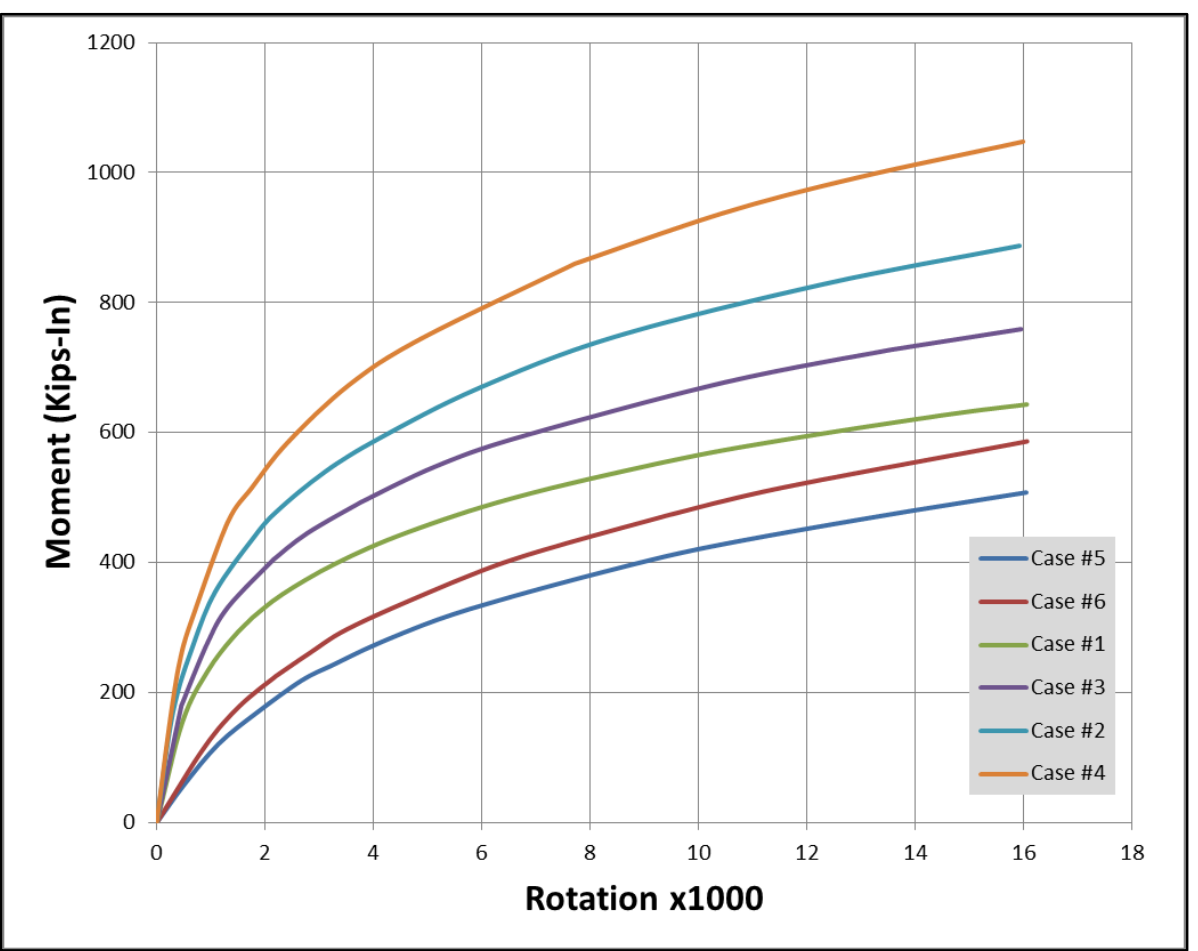

Figure 3-9. Nonlinear moment-rotation response of the semi-rigid connections

Table 3-1. Compression between story displacement responses obtained in this study and Azizinamini's

\begin{tabular}{|c|c|c|c|c|c|c|c|c|c|c|c|c|}
\hline & \multicolumn{2}{|c}{ Fixed } & \multicolumn{2}{c|}{ Pinned } & \multicolumn{2}{c|}{ Case 1 } & \multicolumn{2}{c|}{ Case 4 } & \multicolumn{2}{c|}{ Case 5 } & \multicolumn{2}{c|}{ Case 6 } \\
\hline & *OAM & $* * \mathrm{Az}$ & OAM & $\mathrm{Az}$ & OAM & $\mathrm{Az}$ & OAM & Az & OAM & Az & OAM & Az \\
\hline ST-1 & 0.256 & 0.25 & 1.12 & 1.12 & 0.378 & 0.38 & 0.313 & 0.32 & 0.472 & 0.48 & 0.454 & 0.45 \\
\hline ST-2 & 0.547 & 0.54 & 3.56 & 3.56 & 0.900 & 0.9 & 0.706 & 0.72 & 1.211 & 1.23 & 1.150 & 1.14 \\
\hline ST-3 & 0.697 & 0.69 & 6.42 & 6.42 & 1.221 & 1.23 & 0.922 & 0.95 & 1.771 & 1.8 & 1.658 & 1.64 \\
\hline
\end{tabular}

*OAM: OpenSEES Analytical Model

**Az: Aziznamini’s 


\subsection{Validation of Analytical Approach Using 5-Story Shake Table Results}

Toward comprehensive validation of the analytical approach and dynamic simulation method, nothing would be more helpful than simulating full-scale shake table tests of multi-story buildings and comparing the estimated responses with actual shake table results. To this end, two full-scale shake table test conducted on 5-story and 4-story moment frame buildings were simulated in this study. This section presents the simulation of a shake table study on a 5-story building and the simulation of a 4-story building is presented in the following section.

In 2011, an earthquake simulation was performed on a full-scale 5-story moment frame building in the E-Defense facility, one of the largest shake table facilities in the world located in Miki, Japan. The shake table study was performed in order to investigate the dynamic response due to the implementation of two different seismic isolation systems (Ryan, Dao, Sato, Sasaki, \& Okazaki, 2012). As a reference case, a fixed-base moment fame shake test was also conducted which was used in this study for validation analysis.

The tested building was an asymmetric 5-story structure with two bays in both directions, having staircase located in a corner and other detailing shown in Figure 3-10. The floor system was composite steel beam and concrete slab. The test information including documentation, drawing and excel sheet results all acquired from the NEES database (https://nees.org). The analytical model was developed using OpenSEES finite element software and the modeling approach as described in Chapter II. It should be added that the beam capacities in negative and positive moment were calculated by considering the composite action and also the beam stiffness was doubled to incorporated the composite action effect. 

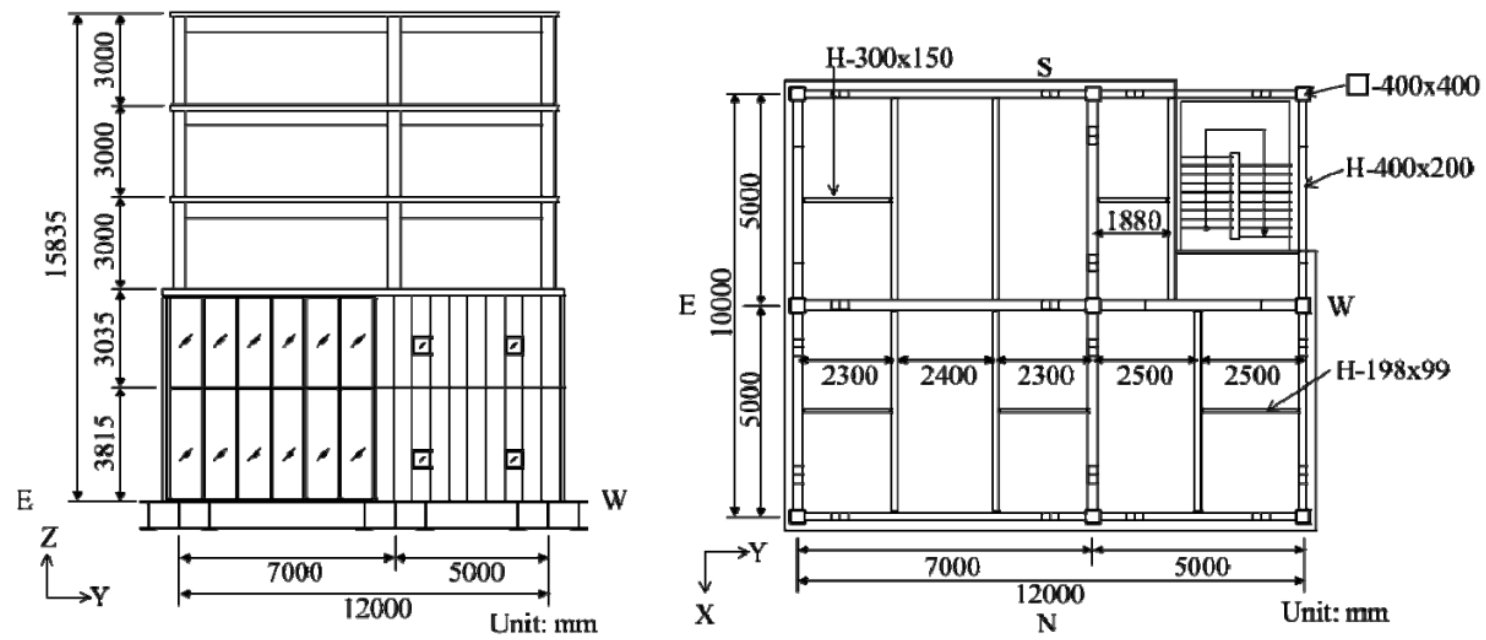

Figure 3-10. Details of the 5-story steel moment frame building (Ryan, Dao, Sato, Sasaki, \& Okazaki, 2012)

Figure 3-11 shows the first five mode shape and the natural periods obtained from both shake table measurements by conducting white noise tests as reported by Dao and Ryan (2013), and as estimated in the analytical simulation. Comparing the test and modal analysis output indicates a good agreement between the results.

\begin{tabular}{|c|c|c|c|c|c|}
\hline & & & & & \\
\hline & & & & & \\
\hline
\end{tabular}

Figure 3-11. Modal shape and natural periods of the 4-story building obtained from both the test and the analytical simulation

The response of the building to 35\% of three-component 1994 Northridge Earthquake at Rinaldi receiving station was simulated by considering $2 \%$ damping incorporated into the model using viscous Rayleigh damping model. The peak drift ratio response obtained from the analytical 
model in two principle directions of the building were compared with shake table results as shown in Figure 3-12. Comparing the results indicates an acceptable agreement which verifies the implemented analytical approach for the linear dynamic response.

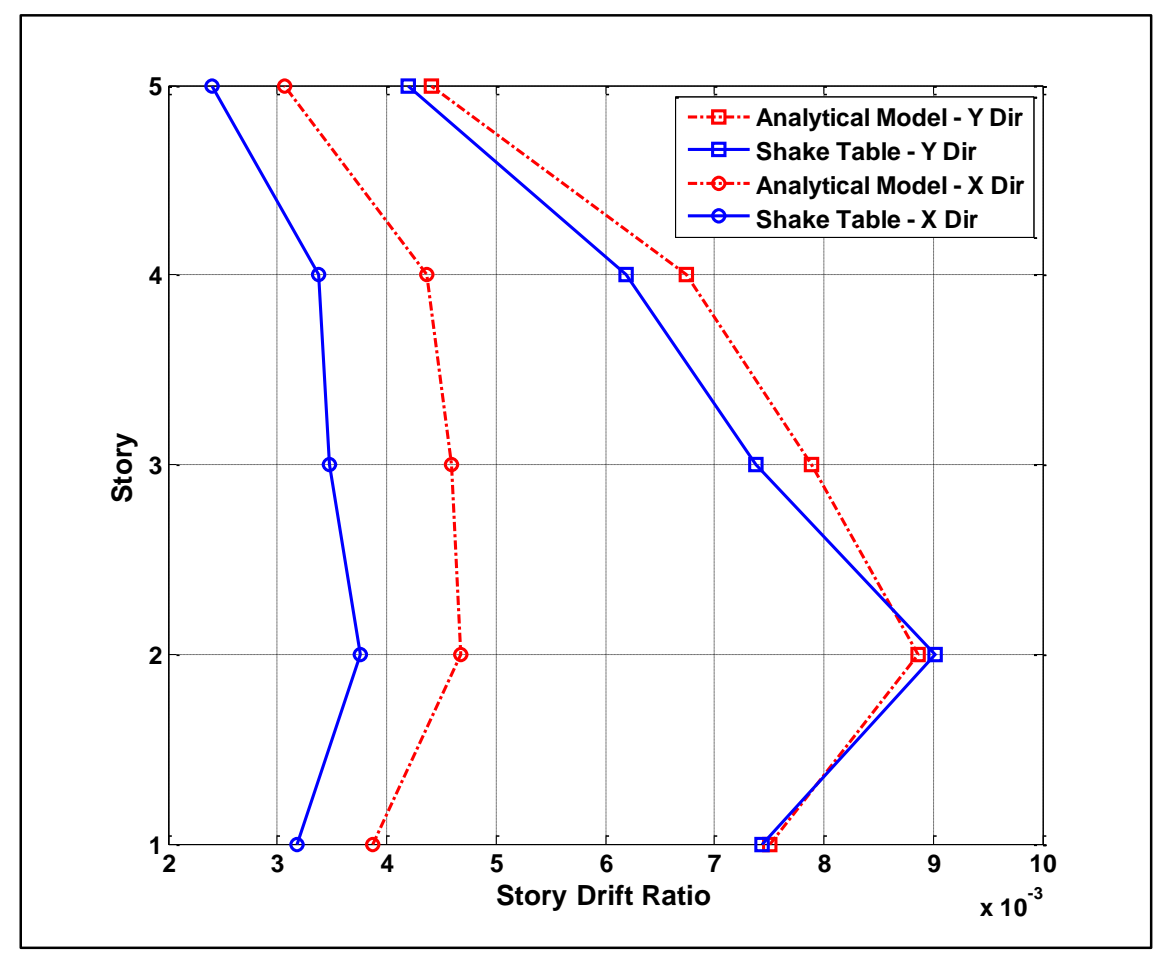

Figure 3-12. Peak story drift ratio of the 5-story building subjected to 35\% Northridge earthquake; the test results versus the analytical simulation results.

The same 5-story building model was used to investigate the validation of developed 3D parallelogram model used to simulate nonlinearity of panel zones in the $3 \mathrm{D}$ model. While the model consisted of a large number of nodes and rigid elements and restrained and released degrees of freedoms to simulate 3D shear deformation of the panel zones, it was a concern that developed model may result in unrealistic deformation. Therefore, an investigation was performed by conducting a pushover analysis in both the $\mathrm{X}$ and $\mathrm{Y}$ directions of the building using a first mode loading pattern. Theoretically, the developed 3D panel zone model provided with very high stiffness model results in the same response for rigid offset model where the panel zone area is modeled with absolute zero deformation. As shown Figure 3-13 the high-stiffness 3D 
panel zone model is compared with rigid offset models developed in OpenSEES and SAP2000.

The figure shows good agreement which proves the reliability of the developed panel zone model.

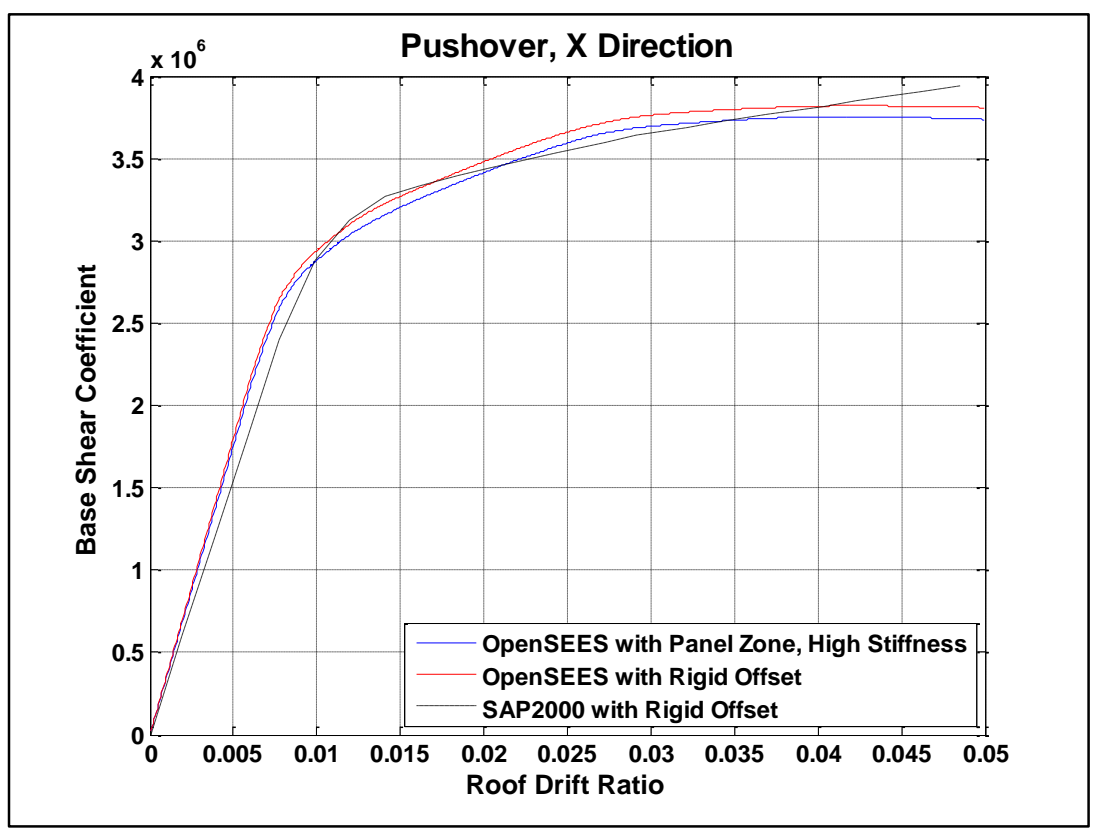

(a)

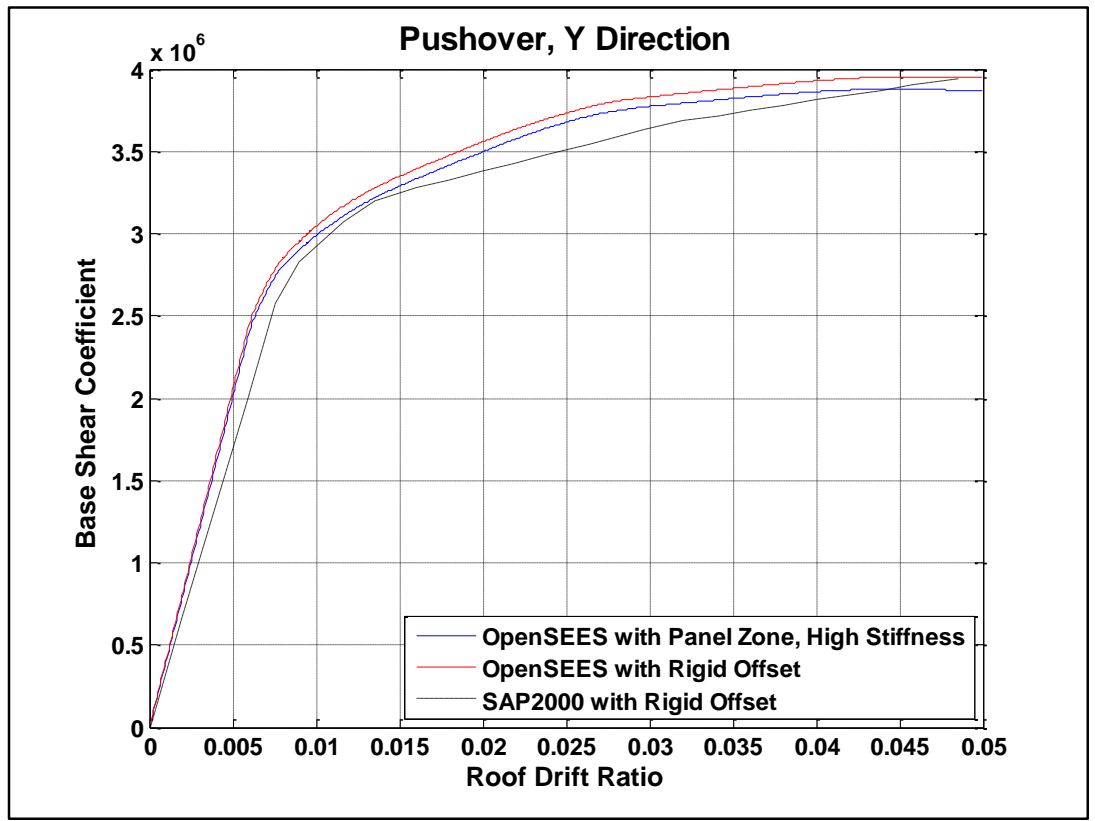

(b)

Figure 3-13. Pushover results for different methods of panel zone modeling: (a) In X-Direction, (b) in Y-direction 


\subsection{Validation of Analytical Approach Using 4-Story Shake Table Results}

The full-scale shake table test of the 5-story moment frame building as described in the previous section did not extend the dynamic response of the building to the nonlinear range. Therefore, the conducted simulation did not challenge the dynamic nonlinearity modeling approach. To evaluate the dynamic nonlinear analytical approach, other sets of shake table tests were simulated, where the responses associated with significant nonlinear dynamic response were experimented with.

In 2007, a full-scale 4-story steel special moment frame building, with details shown in Figure 3-14, was tested in the E-Defense shake table facility. The building was subjected to increasing scaled intensities of three-dimensional ground motion of the1995 Kobe earthquake up to the overall collapse of the building. This experimental study was used to validate the developed nonlinear dynamic analytical approach by comparing the estimated responses with actual shake table results. To this end, all the test information including documentation, drawing and excel sheet results were acquired from NEES database (https://nees.org).

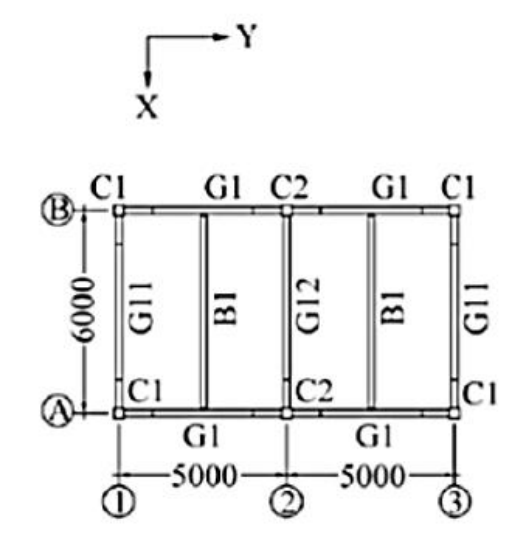

Unit: $\mathrm{mm}$

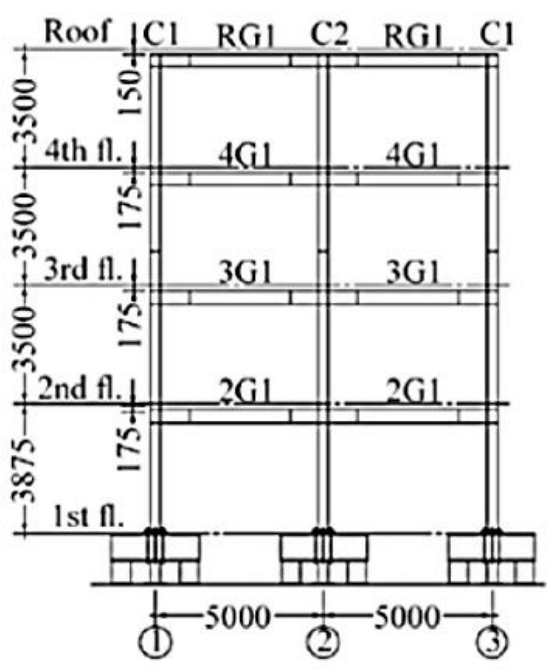

(b) Y-elevation

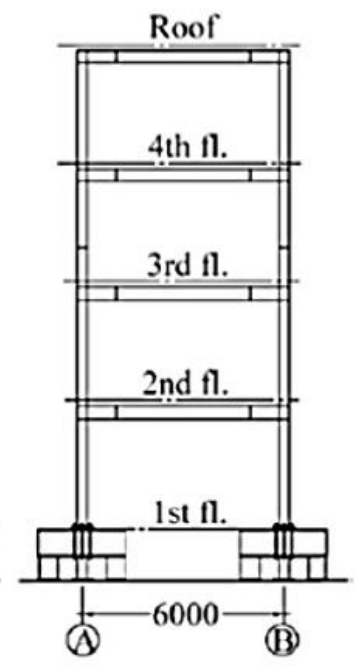

(c) X-elevation

Figure 3-14. Details of the 5-story steel moment frame building 
The 4-story building was modeled in OpenSEES using the modeling approach described in Chapter II and the same method to incorporate composite action effects as described for a 5-story building shake table test model. The estimated modal responses including the mode shapes and natural periods of the first five modes are presented in Figure 3-15. The figure also provides a comparison with actual natural periods of the two first modes measured through white noise shake table tests in two principle directions of the building. A good agreement between the estimated and measured natural periods was obtained.

\begin{tabular}{|c|c|c|c|c|}
\hline & & & & \\
\hline
\end{tabular}

Figure 3-15. Modal shape and natural periods of the 4-story building obtained from both the test and the analytical simulation

In this shake table study, the full-scale 4-story building was tested with different scaled 1995 Kobe earthquake ground acceleration recorded at Takatori station. The unscaled ground acceleration in three dimensions, including two horizontal and the vertical components, are presented in Figure 3-16. Table 3-2 shows the different intensities of scaled ground motion applied in the shake table testing and corresponding building's response states and design stages. In this study, four cases including $0.1,0.2,0.4$ and 0.6 intensity scales containing both elastic and inelastic responses, were simulated. Estimated Peak story drift responses in two given $\mathrm{X}$ and $\mathrm{Y}$ directions are compared with actual recorded shake table responses as shown in Figure 3-17. The comparison concludes acceptable accuracy in predicting the shake table results in both elastic and inelastic range which generally validates the implanted analytical approach. 


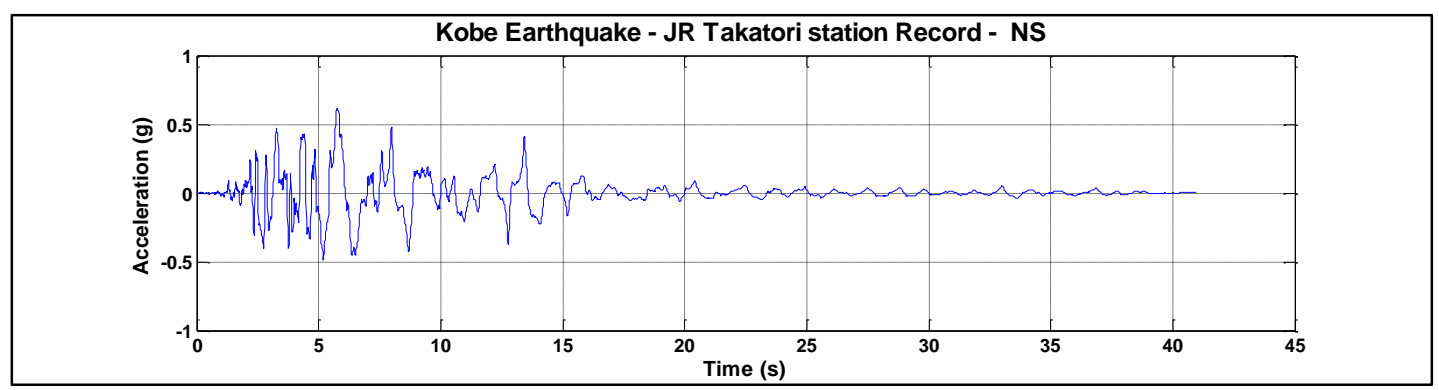

(a)

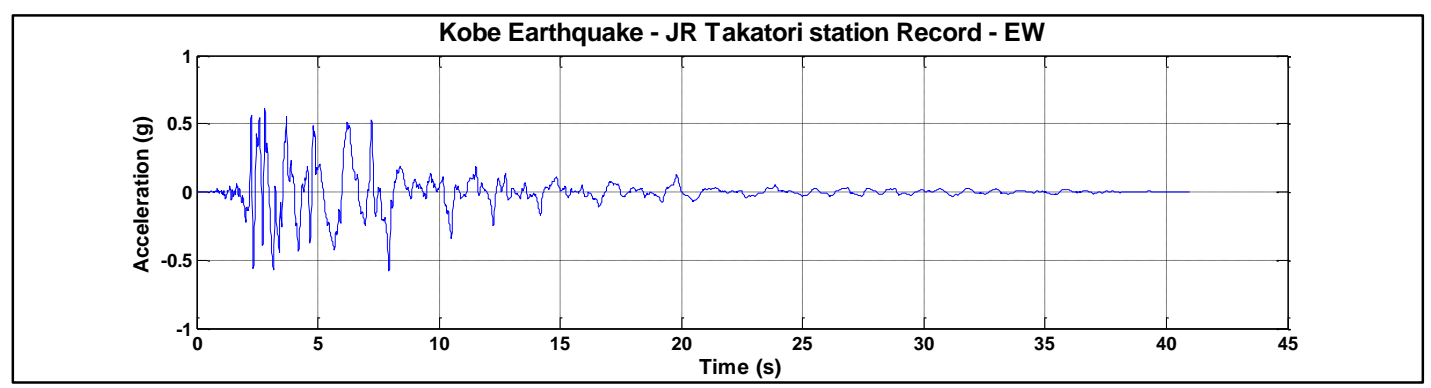

(b)

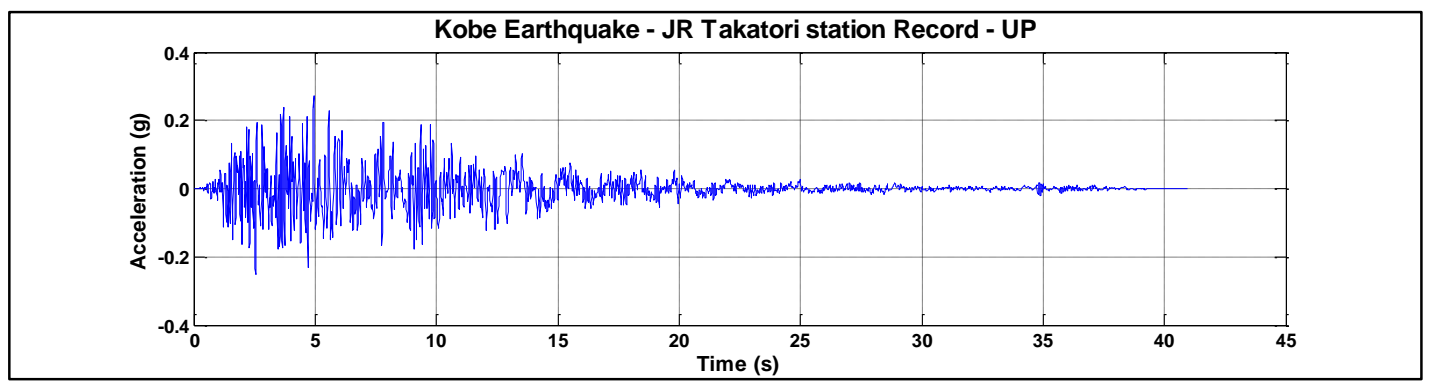

(c)

Figure 3-16. 1995 Kobe earthquake ground acceleration recorded at Takatori Station: (a) in N-S direction; (b) in E-W direction; and (c) in vertical Direction 
Table 3-2. Intensities of the applied excitations and corresponding building's response states and the design stages

\begin{tabular}{|c|c|c|c|}
\hline $\begin{array}{c}\text { Number of the } \\
\text { excitation }\end{array}$ & $\begin{array}{c}\text { Amplification } \\
\text { factor }\end{array}$ & $\begin{array}{c}\text { State of the } \\
\text { specimen }\end{array}$ & \\
\hline 1 & 0.05 & elastic & \\
\hline 2 & 0.1 & elastic & \\
\hline 3 & 0.125 & elastic & \\
\hline 4 & 0.2 & elastic & $\begin{array}{c}\text { corresponding to the design } \\
\text { earthquake level-1 }\end{array}$ \\
\hline 5 & 0.4 & elasto-plastic & $\begin{array}{c}\text { corresponding to the design } \\
\text { earthquake level-2 }\end{array}$ \\
\hline 6 & 0.6 & elasto-plastic & \\
\hline 7 & 1.0 & collapse & \\
\hline
\end{tabular}




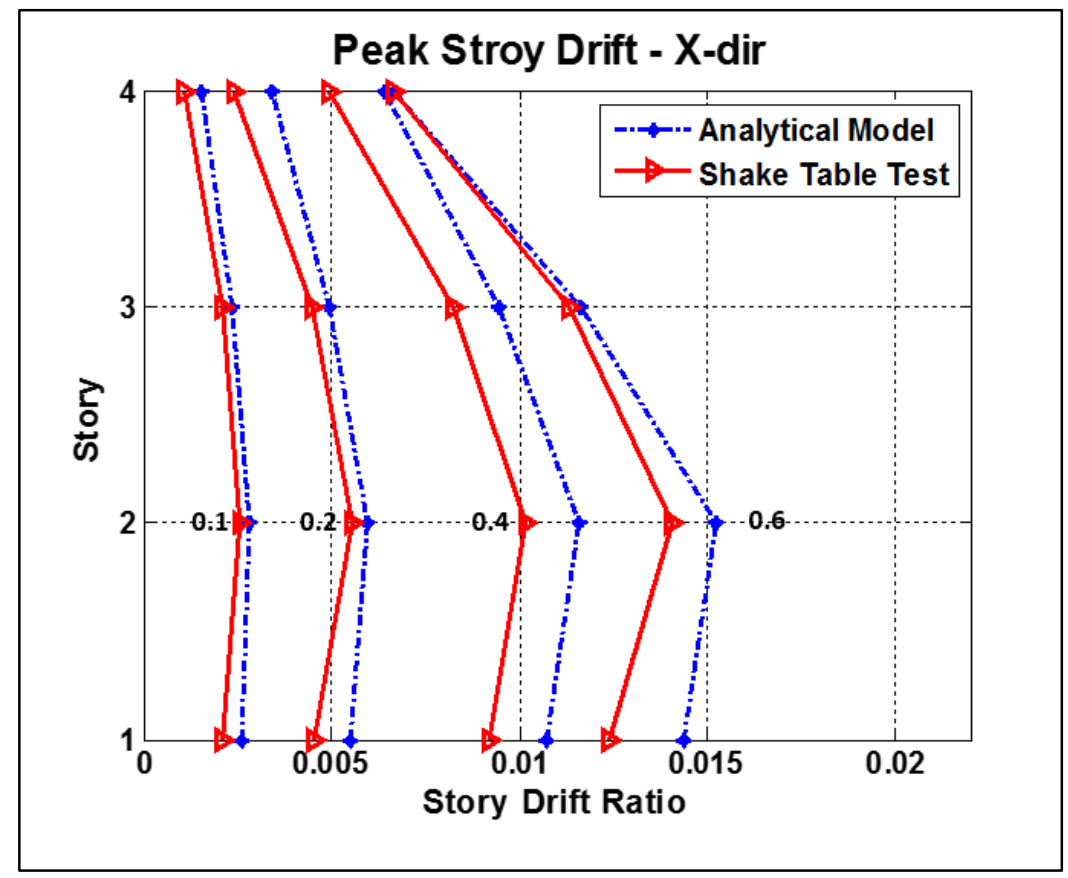

(a)

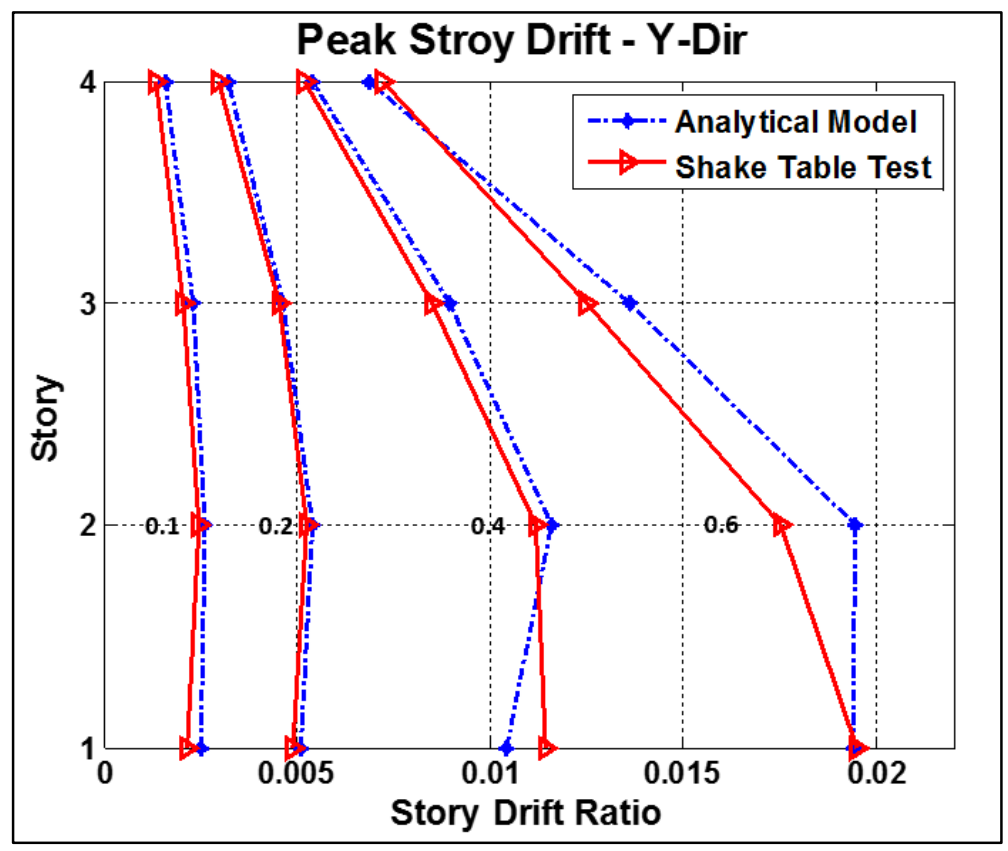

(b)

Figure 3-17. Peak story drift ratio of the 4-story building subjected to different intensities of Kobe earthquake; test results versus analytical simulation results: (a) in X-direction; (b) in $Y$ direction 


\section{CHAPTER IV}

\section{STUDY ON ANALYTICAL MODELING OF DAMPING}

\subsection{Introduction}

In structural analysis, damping is a term for describing the energy dissipation mechanisms in a structure due to dynamic responses of structural and nonstructural components leading to a reduction in vibration intensity with time. In general, damping of a building is considered as the combination of three damping components (NEHRP, 2004):

- Inherent Damping: Inherent damping of a building in its elastic vibration. The major source of inherent damping is the internal friction in the structural materials, connections, and nonstructural components.

- Hysteresis Damping: damping corresponding to the dissipated energy in the post-yielding nonlinear response of structural members of the lateral resisting system of the building.

- Added Damping: different damping systems (such as isolators, passive and active dampers and etc.) added to the building to enhance the damping characteristics and accordingly improve the performance of the building to dynamic lateral loads such as seismic and wind loads.

In response history analysis, Hysteretic energy dissipations of structural are considered directly with inelastic elements. The inherent damping, according to the type of the structure needs to be properly considered. Due to its friction basis, inherent damping is mainly displacement dependent instead of being velocity-dependent as viscous dampers are. Therefore, a frictional damping model is the most appropriate model to represent the inherent damping. But the complication and nonlinearity associated with the frictional model have led to simplifying the inherent damping with equivalent linear viscous damping (Charney, 2008). 
The general equation of motion for a multi-degree of freedom system is as following:

$$
M \ddot{u}(t)+C \dot{u}(t)+f_{S}(u(t))=P(t)
$$

where $\ddot{u}(t), \dot{u}(t)$ are dynamic acceleration and velocity vectors respectively. $\mathrm{M}$ and $\mathrm{C}$ are the mass and damping matrices. $f_{S}(u(t))$ is a vector relating the resisting force and the displacement. For a linear system $f_{s}(u(t))=K u(t)$ where $\mathrm{K}$ is the stiffness matrix. $P(t)$ is the vector of dynamic load.

Representing the damping depends on the approach implemented to solve the dynamic equilibrium equations. In modal superposition analysis, viscous damping ratios for each mode can be directly implemented into analysis without the need to provide the full damping matrix. However, the damping matrix can be recovered. In this method, the generated damping matrix will be diagonalized (classical damping matrix (Gavin, 2014)). It is most efficient in the analysis of linear systems, while for an n-degree of freedom system, the equation of motion can be simplified to $n$ uncoupled equations.

The Equivalent Linear viscous damping model is also commonly used in the direct integral analysis of nonlinear systems with fully coupled equations. In some cases, particularly in nonlinear systems, using equivalent linear damping models may cause inaccurate response predictions. These inaccurate responses are due to generation of unrealistic damping forces in some nodes during dynamic analysis (Zareian \& Medina, 2010).

Improper modeling of structural damping results in the inadequate estimation of force and deformation demands and consequently leads unreliable structural assessment and design procedures. 


\subsection{Linear Viscous Damping Models}

Linear viscous damping models are usually used to generate the damping matrix $C$ in dynamic analysis of Multi-degree-of-freedom systems such as multi-story buildings. Three commonly used linear viscous damping models are explained in following:

\section{- Rayleigh Damping}

In this model, the classical damping matrix $C$ is composed of a linear combination of mass and stiffness matrices (Chopra, 1995).

$$
C=\alpha M+\beta K
$$

The $\alpha$ and $\beta$ coefficients can be determined from specifying two damping ratios $\zeta_{i}$ and $\zeta_{j}$ for $i$ th and $j$ th modes. The damping ratio for other modes varies with curve and the equation shown Figure 4-1a. As shown in the figure, the damping ratios for natural frequencies lower than $\omega_{i}$ and higher than $\omega_{j}$ increase sharply. It gives emphasis to the importance of including all major participating modes in the frequency range chosen to determine coefficients. The physical equivalent of Rayleigh damping model is to have a system externally supported by mass-proportional dampers (MPDs) at corresponding inertial degrees of freedom and the presence of stiffness-proportional dampers (KPDs) joining two adjacent degree of freedoms as illustrated in Figure 4-1b (Zareian \& Medina, 2010). 


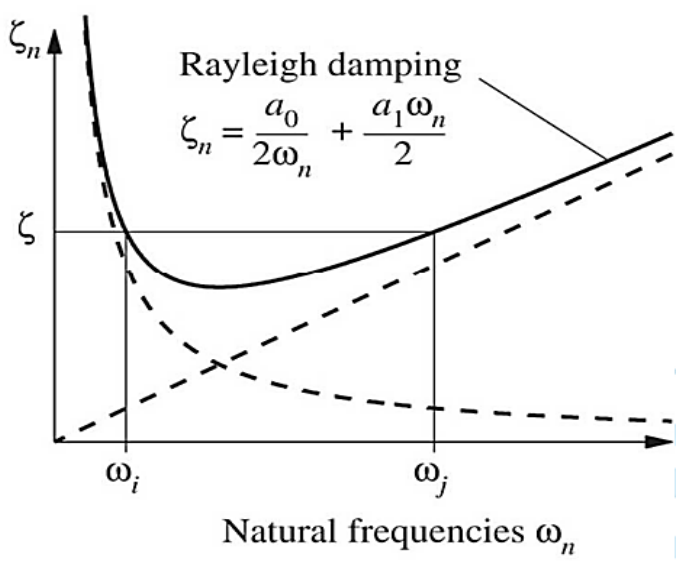

(a)

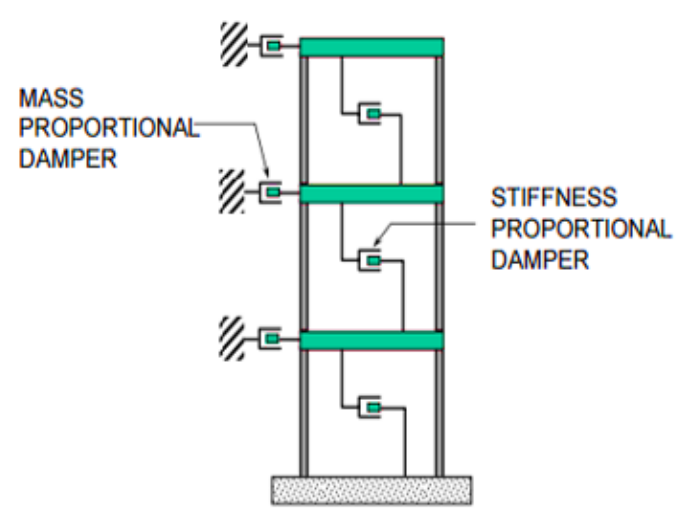

(b)

Figure 4-1. Rayleigh damping: (a) variation of modal damping ratio with natural frequencies; (b) illustration of MPD and KPD component damping.

- Caughey Damping

This model is a generalization of Rayleigh's damping model where a classical damping matrix $C$ can be generated by specifying damping ratios for more than two modes. In this method, the general term of the damping matrix is represented as following equation (Coughey \& Kelley, 1960):

$$
C=M \sum_{b} a_{b}\left[M^{-1} K\right]^{b}
$$

In above equation as many terms as needed with arbitrary coefficients $a_{b}$ can be used. In most general representation of the damping matrix the number of terms is equal to $\mathrm{N}$, the number of DOF. Using more than two terms would result in a bandwidth greater than that of the stiffness matrix which may consume a large solution time (Charney, 2008).

- Superposition of Modal Damping Matrices

The alternative approach to generate the damping matrix is to superimpose all modal damping matrices. The general term of the damping matrix is represented as follows (Chopra, 1995): 


$$
C=M\left(\sum_{n=1}^{N} \frac{2 \zeta_{n} \omega_{n}}{m_{n}} \phi_{n} \phi_{n}^{T}\right) M
$$

where the $n$th term represents the contribution of the $n$th mode with frequency, damping ratio, Eigenvector and the modal mass equal to $\omega_{n}, \zeta_{n}, \phi_{n}$ and $m_{n}$ respectively.

\subsection{Inaccurate Dynamic Response of Nonlinear System from Rayleigh Damping Model}

Several studies in past few years investigated the unrealistic forces and consequently inaccurate responses of nonlinear systems using the Rayleigh damping model. It is shown that, using the Rayleigh damping model proportional to mass and initial stiffness matrices, may lead to generation of unrealistic damping moments at joints where structural elements undergo an abrupt change in stiffness due to yielding (Bernal, 1994; Medina \& Krawinkler, 2004; Hall, 2006; Charney, 2008; Zareian \& Medina, 2010 and Chopra \& McKenna, 2016). These spurious damping forces are due to increase of effective damping to several hundred percents in lower modes (Charney, 2008). This error will lead to an underestimation of the peak displacement response in the structure as well as the collapses potential of the building and an overestimation of internal forces for elements with no significant change in their stiffness (Zareian \& Medina, 2010).

Alternatively, the Rayleigh damping model can be used proportional to the tangent stiffness of the system updating in each step of the analysis. Although this approach is computationally more expensive, is more appropriate and results in more realistic responses (Zareian \& Medina, 2010). Charney (2008) shows that using the Rayleigh damping model proportional to mass and tangent stiffness matrices, when the coefficients are set based on initial stiffness, will lead to moderate spurious damping forces, while setting coefficients based on tangent stiffness removes the errors and no spurious damping forces will develop. 


\subsection{Suggestions for Proper Damping Modeling for Inelastic Systems}

Based upon the conducted studies on modeling the damping in inelastic systems several solutions have been suggested to alleviate or reduce spurious damping forces. Bernal 1994 suggests to assemble the damping matrix by using a stiffness matrix condensed to the size of inertial DOFs, more practically a full DOF stiffness matrix with a set of zero rows and columns for massless DOFs. Hall (2006) suggests eliminating the mass proportional contribution in assembling damping matrix $(\alpha=0)$ to eliminate spurious damping forces associated with seismic rigid body motion response and bound stiffness proportional damping with appropriate caps based on dominant non-linear mechanism.

Charney (2008) suggests excluding nonlinear elements while providing initial stiffness proportional damping when assembling the damping matrix based on the initial stiffness of the system. Assembling the damping matrix based on the tangent stiffness of the system is also recommended to obtain more realistic responses. In this approach, it is suggested to use reduced frequencies to determine the coefficient to provide limited damping in lower modes in the nonlinear stage.

Chopra and McKenna (2016) recommend generating the damping matrix by superposition of modal damping matrices as the most reliable solution to completely eliminate the spurious damping forces. This solution is regardless of the number of modes included or values assigned to modal damping ratios. The study compared responses predicted using three damping models: (1) Rayleigh damping based on initial stiffness, (2) Rayleigh damping using tangent stiffness; and (3) superposition of modal damping matrices. The results indicated that compared to the superposition of modal damping model, Rayleigh damping based on initial stiffness led to unrealistic results, and Rayleigh damping using tangent stiffness resulted in improved results. However, Rayleigh damping proportional to the tangent stiffness matrix is not recommended. 


\subsection{Investigation on Damping Modeling in The 47-Story Building Model}

State-of-the-art findings in the literature strongly recommend using superposition of modal damping matrices to alleviate spurious damping forces. But for Wind Analysis of a high-rise building with very large 3D model and long wind analysis duration (around one hour), the implementation of this method was not feasible. Therefore, an investigation was performed to compare results obtained from the different possible approach of Rayleigh damping and results from the superposition of modal damping method for a free vibration analysis and most reliable method of Rayleigh damping was selected for the wind nonlinear time-history analyses.

\section{Rayleigh Damping Method in OpenSEES}

The Rayleigh damping assigned to all elements and nodes (except zero-length elements and truss elements) is expressed as following equation.

$\mathrm{C}=\$$ alphaM $* \mathrm{M}+\$$ betaK $*$ Kcurrent + \$betaKinit $*$ Kinit + \$betaKcomm $*$ KlastCommit

where \$alphaM is the factor applied to the element or node mass matrix M. \$betaK is the factor applied to the current element stiffness matrix Kcurrent. \$betaKinit is the factor applied to the initial element stiffness matrix Kinit. And finally, \$betaKcomm is the factor applied to the last committed element stiffness matrix KlastCommit. It indicates that the model is flexible to present the damping either based on initial stiffness matrix or current stiffness matrix or last committed stiffness matrix or any combination of them. In this investigation the free vibration responses of the 47-story building using four following damping models were obtained:

1) Rayleigh damping based on initial stiffness matrix

2) Rayleigh damping based on current stiffness matrix

3) Rayleigh damping based on last committed stiffness matrix

4) superposition of modal damping matrices 


\section{FREE VIBRATION ANALYSES}

Free vibration analyses in both elastic and inelastic responses for models using four mentioned damping models were performed. For free vibration analysis, first the building was statically pushed by scaled ASCE 7-10 wind load (two scale factors corresponding to elastic and inelastic response were used) and then the load was removed and it followed by a dynamic free vibration analysis.

\section{- Elastic Free Vibration}

For elastic free vibration analyses, the building was pushed statically by $10 \%$ of ASCE 7-10 wind loading of the building and then by removing the load free vibration analyses were performed with the four different damping models: Rayleigh damping based on initial stiffness matrix, current stiffness matrix, and last committed stiffness matrix and finally superposition of modal damping matrices. For all damping models, $2 \%$ damping ratio was considered. In Rayleigh damping models, the $1^{\text {st }}$ and $20^{\text {th }}$ modes were used to calculate mass and stiffness matrix coefficients. In the method using superposition of modal damping matrices, 20 modes contributed to assemble the damping matrix.

Figure 4-2 shows the Roof displacement responses to free vibration for the four different 47-story building models using different damping assumption. The figure indicates coincident responses for all damping assumption to elastic free vibration. As recommended by literature, in the elastic range different damping methods as investigated in this study such as Rayleigh damping models, Rayleigh damping based on initial stiffness matrix, current stiffness matrix and last committed stiffness matrix and finally superposition of modal damping matrices, all resulted in the same response for the 47 -story building model. 


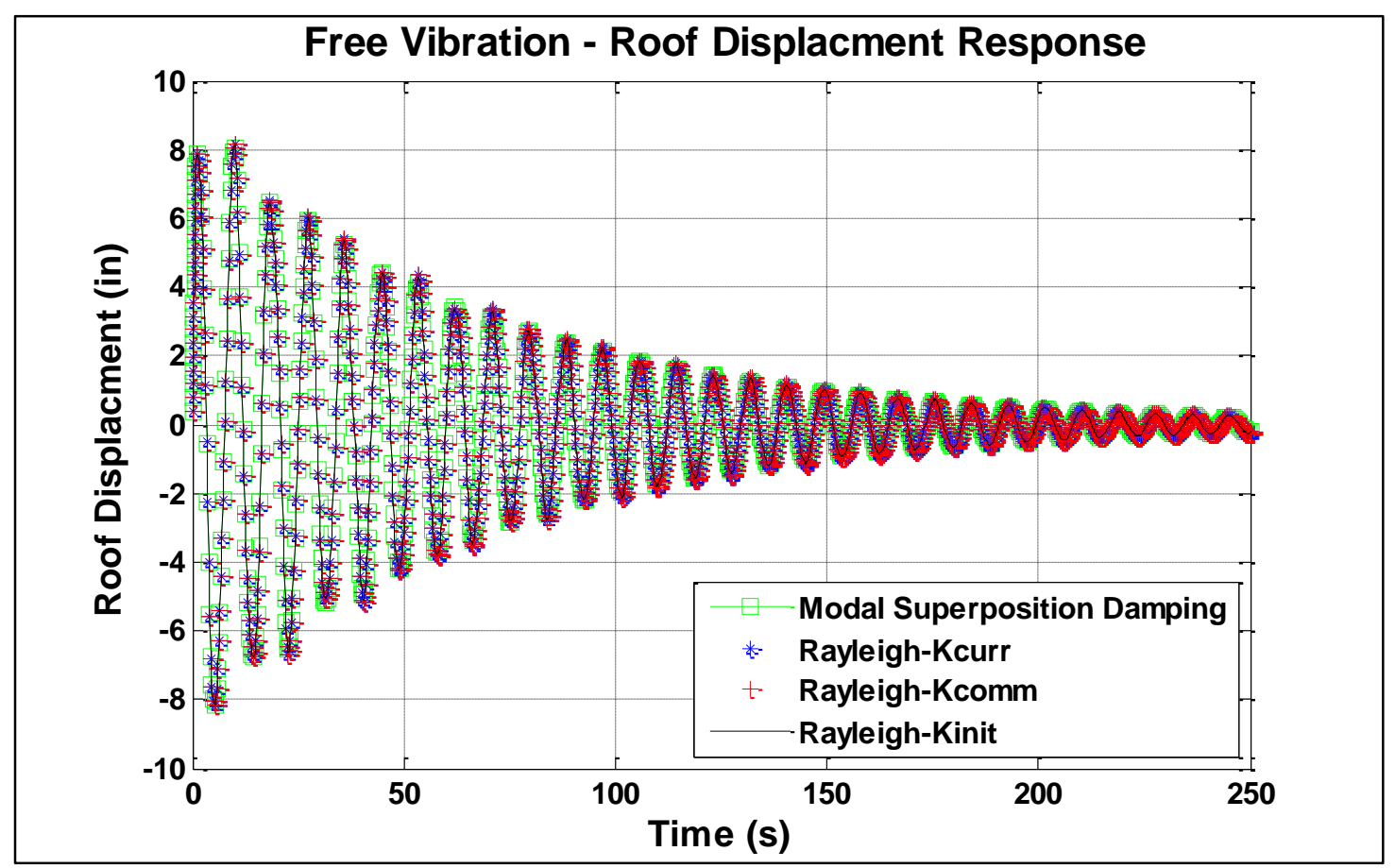

Figure 4-2. Elastic free vibration; the roof displacement responses for the four different damping models

For each damping model, the actual viscous damping ratio was measured from the obtained response to be compared with the assumed damping (2\% of critical damping for all four implemented damping model) and examine if the analytical model properly reflects the assumed damping. To this end, the time history response was first transformed to frequency domain response for recognizing the participation of different modes to free vibration response with initial lateral deformation corresponding to scaled ASCE 7-10 wind loading.

The frequency domain free vibration response of the 47-story building model using Rayleigh damping based on tangent stiffness matrix is shown in Figure 4-3. The figure indicates that the free vibration response with initial sway due to scaled ASCE 7-10 wind loading is dominated by the first mode response of the building in that direction. Natural frequencies obtained from the frequency-domain transformation for all responses corresponding to different damping models are shown in Table 4-1. 


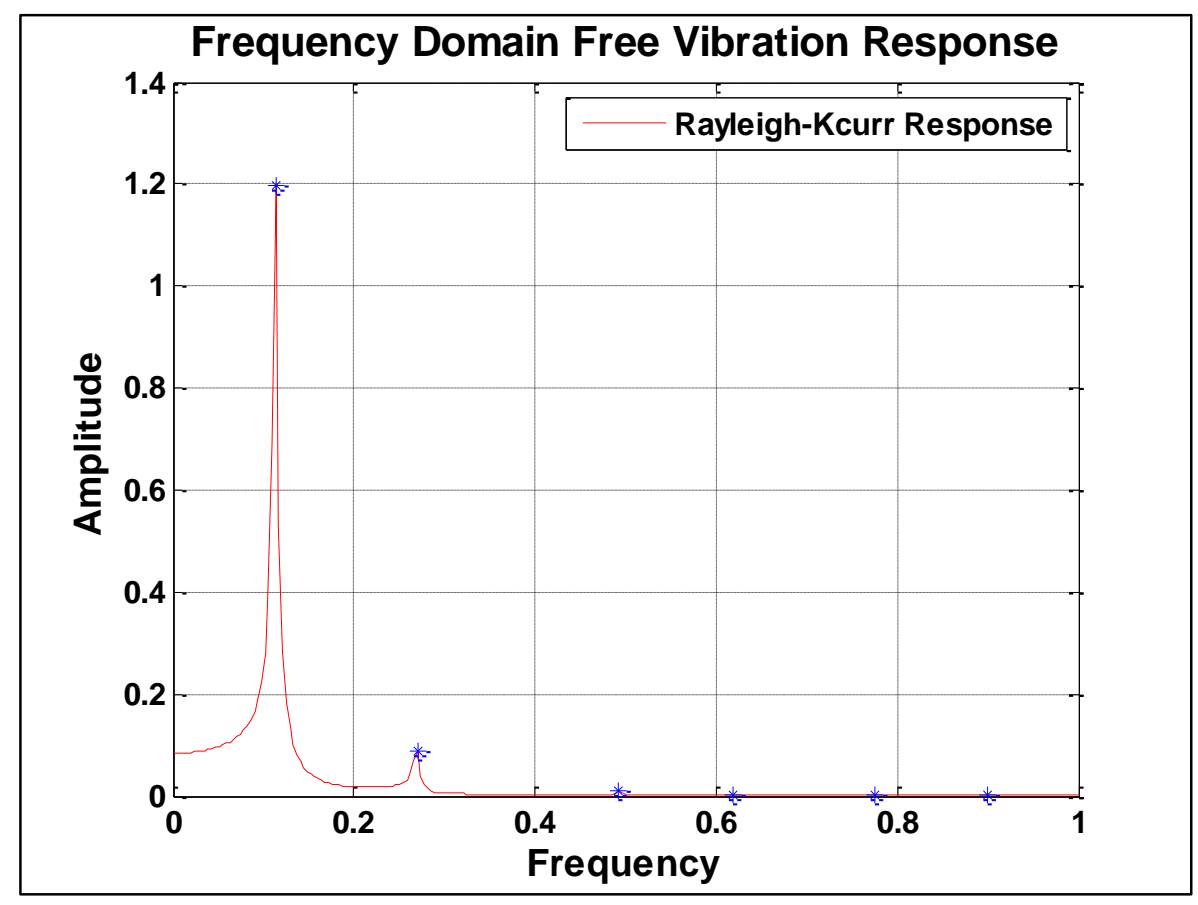

Figure 4-3. Frequency domain elastic free vibration response of the 47-story building; using Rayleigh damping based on tangent stiffness matrix

An exponential trend curve was then fitted to the free vibration response to measure the actual damping of the system. According to dynamic of structure fundamentals, the free vibration response amplitude of each mode of underdamped systems $(\zeta<1)$ decreases by according to $e^{-\zeta \omega_{n} t}$ (Chopra, 1995). Considering the response dominated by the first mode the whole response is considered to by decayed according to $e^{-\zeta \omega_{1} t}$. Comparing it with the obtained fitted exponential curve the equivalent viscous damping coefficient were measured as reported in Table 4-1. Figure 4-4 shows the exponential curve fitted to the free vibration response using Rayleigh damping based on tangent stiffness matrix. 


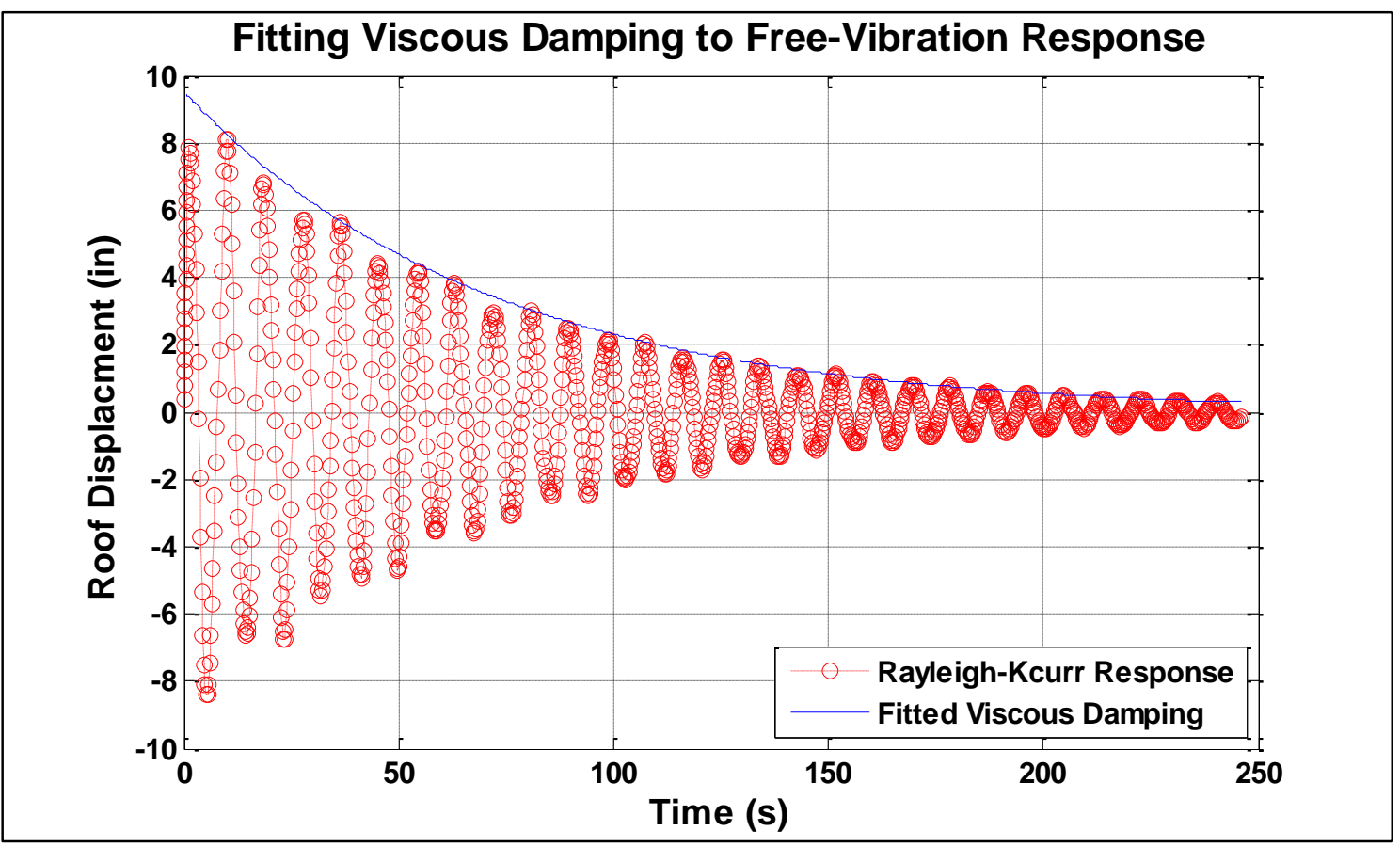

Figure 4-4. Fitted viscous damping to free vibration response of the 47-story building; using Rayleigh damping based on tangent stiffness matrix

\section{- Nonlinear Free Vibration}

For Nonlinear free vibration analyses, the building was pushed statically by $125 \%$ of ASCE 7-10 wind loading of the building. The loading corresponded the development of nonlinearity in structural elements as shown in Figure 4-5. The figure indicates the nonlinearity in a large number of beams as well as a few columns and panel zones. By removing the load free vibration analyses were then performed with the four different damping models: Rayleigh damping based on initial stiffness matrix, current stiffness matrix and last committed stiffness matrix and finally superposition of modal damping matrices. For all damping models, the same damping ratio (2\%) was considered. The same as for elastic free vibration, for Rayleigh damping models, the 1st and 20th modes were used to calculate mass and stiffness matrix coefficients. Also in the superposition of modal damping matrices method, 20 modes contributed to assemble the damping matrix. It also should be noted that the Rayleigh damping was not assigned to nonlinear zerolength springs representing the nonlinearity in all beams, columns, panel zones and semi-rigid 
connections, as it was recommended by several recent studies to avoid development of spurious damping forces (Charney, 2008; Zareian \& Medina, 2010).

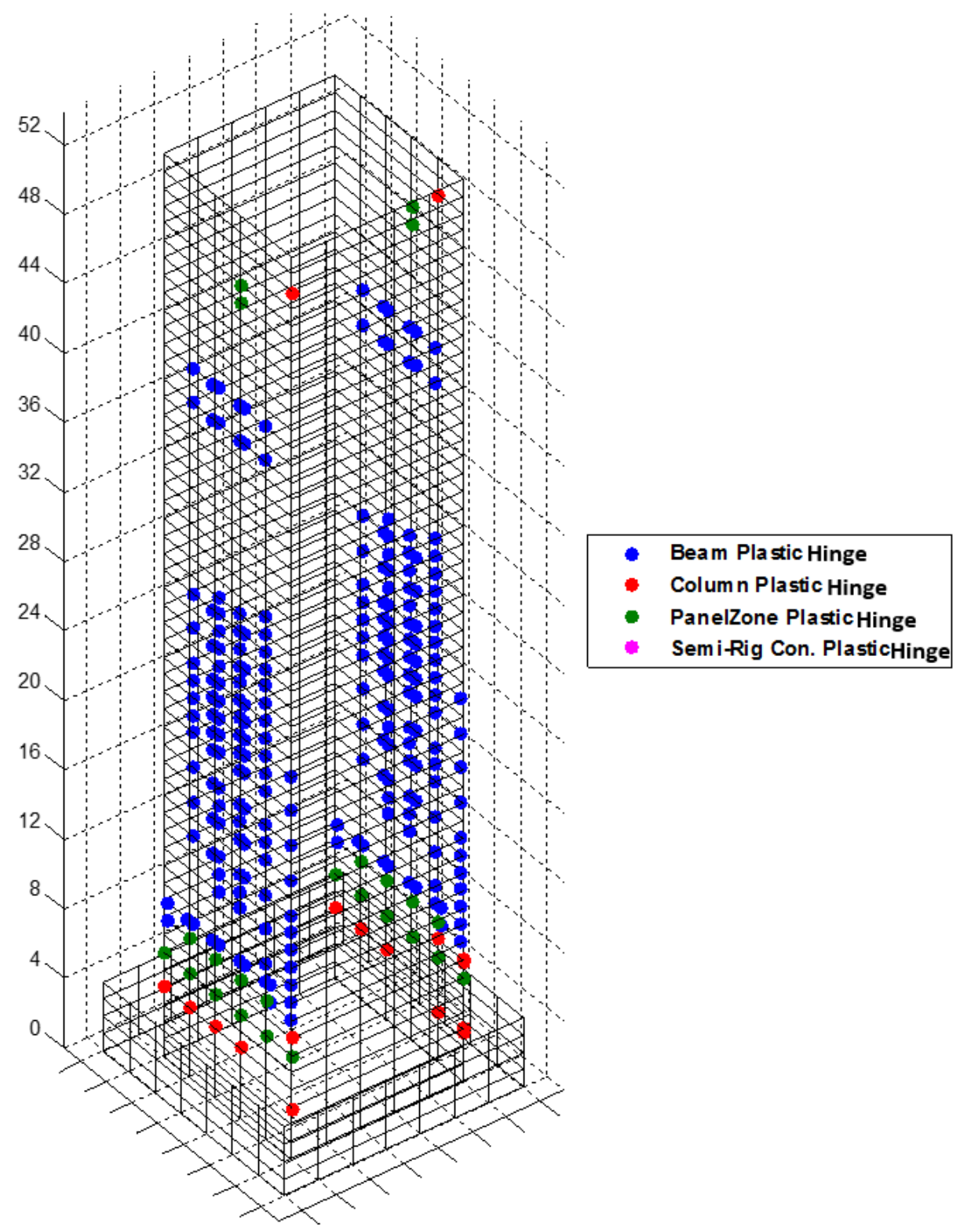

Figure 4-5. Nonlinearity in structural members of the 47-story building due to statically pushing by $125 \%$ of ASCE 7-10 wind loading 
Roof displacement responses to inelastic free vibration for four different 47 -story building models using different damping assumption is shown in Figure 4-6. The figure indicates coincident nonlinear responses for all damping assumption to inelastic free vibration.

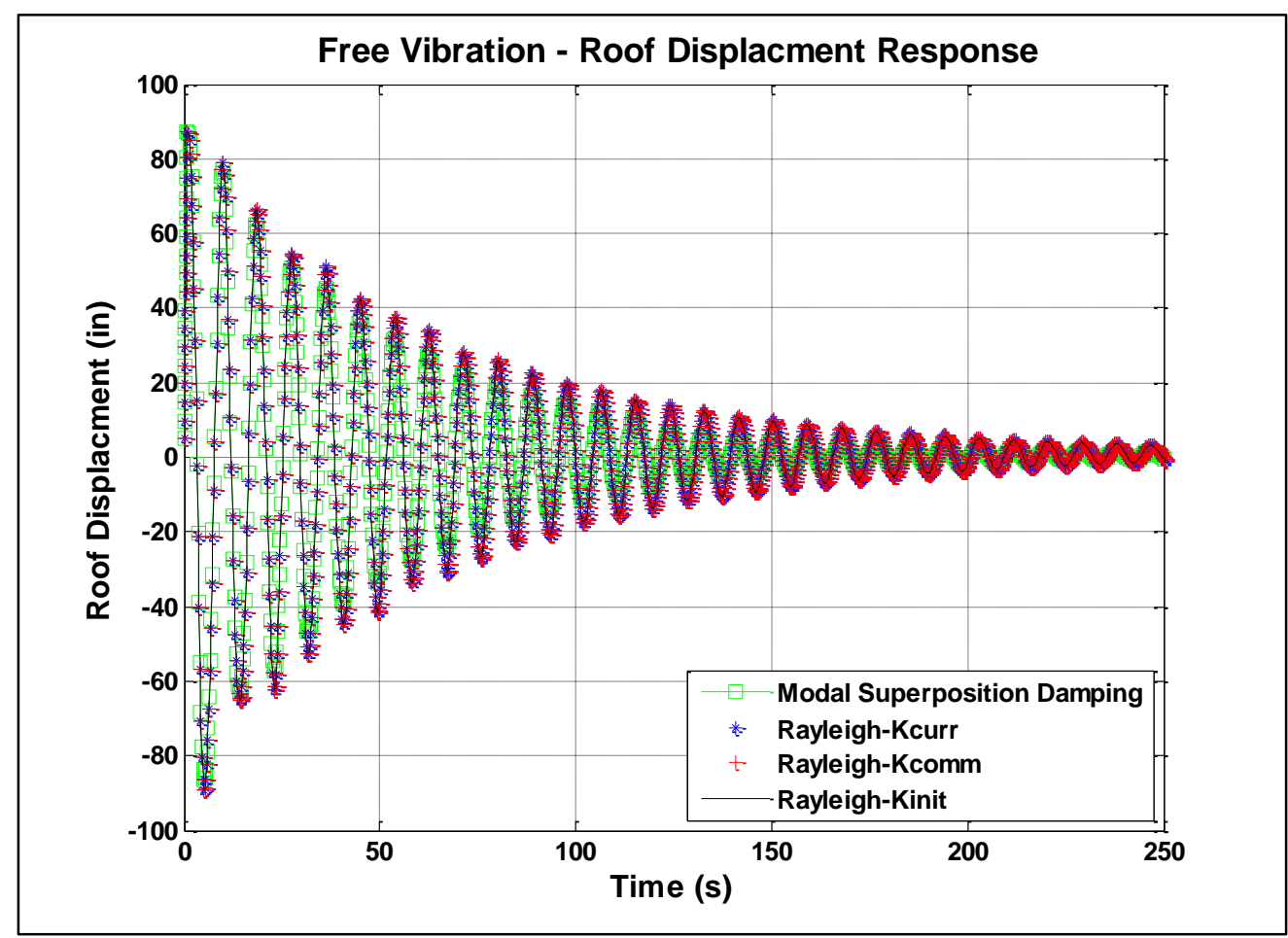

Figure 4-6. Inelastic free vibration; the roof displacement responses for the four different damping models

Figure 4-7 shows frequency domain inelastic free vibration response of the 47 -story building model using Rayleigh damping based on tangent stiffness matrix. The figure indicates that the inelastic free vibration response is also dominated by the first mode response of the building. Natural frequencies obtained from the frequency-domain transformation for all responses corresponding to different damping models are shown in Table 4-1. 


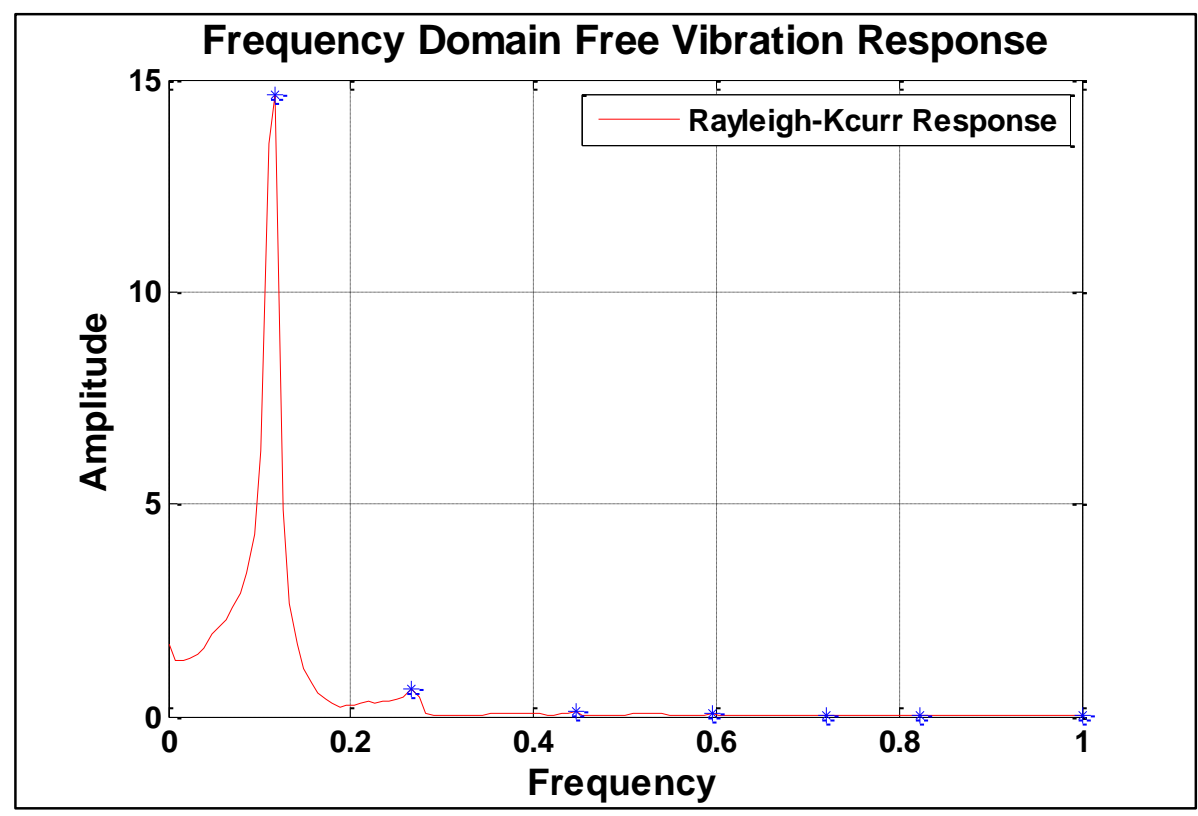

Figure 4-7. Frequency domain inelastic free vibration response of the 47-story building; using Rayleigh damping based on tangent stiffness matrix

As already explained, an exponential trend curve was then fitted to the free vibration response to measure the actual damping of the system. Figure 4-8 shows the exponential curve fitted to free vibration response using Rayleigh damping based on tangent stiffness matrix. The obtained equivalent viscous damping coefficient for inelastic free vibration for models with different damping assumptions is reported in Table 4-1. 


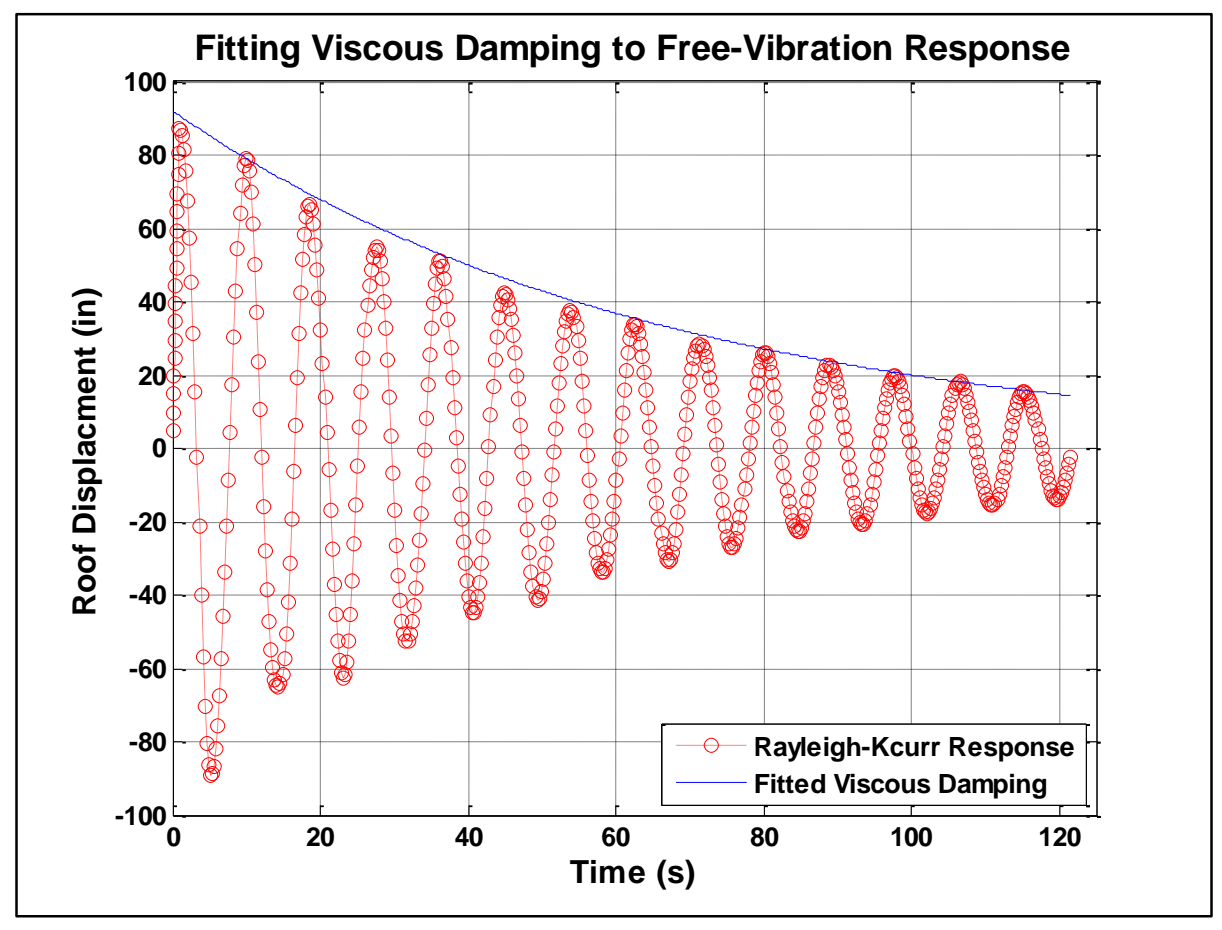

Figure 4-8. Fitted viscous damping to inelastic free vibration response of the 47-story building; using Rayleigh damping based on tangent stiffness matrix

Table 4-1 summarizes the results obtained from both elastic and inelastic free vibration analyses for four models with different damping models: Rayleigh damping based on initial stiffness matrix, current stiffness matrix and last committed stiffness matrix and finally superposition of modal damping matrices. The measured natural frequencies obtained from frequency domain transforms as well as the measured viscous damping coefficients obtained from fitting exponential trend curves for all damping models are shown in the table. 
Table 4-1. Natural frequencies and the viscous damping coefficients obtained from elastic and inelastic free vibration analyses.

\begin{tabular}{|c|c|c|c|c|}
\hline \multirow{2}{*}{ Damping Modeling method } & \multicolumn{2}{|c|}{ Elastic Response } & \multicolumn{2}{c|}{ Nonlinear Response } \\
\cline { 2 - 5 } & Natural Frequency & Damping & Natural Frequency & Damping \\
\hline Rayleigh-Kinit & 0.1174 & 0.0199 & 0.1096 & 0.0273 \\
\hline Rayleigh-Kcomm & 0.1174 & 0.0196 & 0.1096 & 0.0271 \\
\hline Rayleigh-Kcurr & 0.1174 & 0.0196 & 0.1096 & 0.0271 \\
\hline Modal Superposition Damping & 0.1174 & 0.0209 & 0.1096 & 0.0273 \\
\hline
\end{tabular}

\subsection{Discussion and Conclusion}

A comprehensive investigation considering inherent damping in an analytical model of building structures was conducted in this section. The available literature mainly warned about unrealistic damping forces associated with using Rayleigh damping in inelastic systems. Several solutions such as: using Rayleigh damping based on tangent stiffness matrix, excluding nonlinear elements from providing stiffness proportional damping and etc. However, generating a damping matrix by superposition of modal damping matrices was recommended as the most reliable method resulting in the complete elimination of the spurious damping forces.

For a large 3D analytical model, such as 47-story building model developed in this research, using superposition of modal damping matrices method is super time-consuming, which makes it infeasible to use. Thus, the recommended solution of excluding nonlinear elements from providing stiffness proportional damping was used. Elastic and inelastic free vibration analyses were performed using scaled ASCE 7-10 wind loading as the initial condition. Then, the obtained result from using Rayleigh damping (with only stiffness proportional damping for elastic members) was compared with superposition of modal damping matrices method- as the most reliable method with no spurious damping forces - to make sure that the damping is considered properly. 
While Rayleigh damping in OpenSEES can be generated based on initial, tangent and current stiffness matrixes, all three alternatives were included in this investigation. Measured damping from both elastic and inelastic free vibration analyses for all alternative Rayleigh damping methods as well as a superposition of modal damping matrices method are presented in Table 4-1. It is shown that the both elastic and inelastic stages all alternative Rayleigh damping methods (with only stiffness proportional damping for elastic members) resulted in the coincident measured damping as was measured for superposition of modal damping matrices method and they were almost equal to the assumed viscous damping ratio of $2 \%$. It suggests that Rayleigh damping methods with excluding nonlinear members from providing stiffness proportional damping regardless of the type of stiffness matrix (initial, tangent or current stiffness matrix) results in more reliable viscous damping simulation for the 47 -story building model. 


\section{CHAPTER V}

\section{WIND PRESSURE TESTING AND WIND DYNAMIC LOADING}

\subsection{Introduction}

To perform wind nonlinear dynamic analyses, it was required to obtain accurate dynamic wind loadings for all three high-rise building models investigated in this research. For the 47-story building, the dynamic wind loading was acquired by conducting sets of wind pressure testing on a rigid scaled model. For the other two buildings (30- and 40-story buildings) the wind loadings were obtained using the wind tunnel data presented by Tokyo Polytechnic University (TPU) aerodynamic database for high-rise buildings. Conducting the wind pressure testing, also provided the chance to study the characteristics of the wind loading on a high-rise building due to effects of surrounding buildings and building shape characteristics.

The characteristics of wind loading on the 47-story building were studied by conducting a set of wind tests in 12-fan Wall of Wind facility at Florida International University on a rigid 1/400 scale model. Three sets of wind tests were conducted on three models including: (1) an isolated prismatic model; (2) an isolated full building model including shape details (the skirt and the penthouse); and (3) A full building model surrounded by neighboring buildings. The objectives of this experimental study were to obtain realistic wind loading data for the 47 -story building as well as to evaluate the significance of both the interference effect of sheltering buildings and the effect of the shape characteristics on the magnitude and distribution of wind loads on the building. The test results were also compared with TPU wind tunnel test results.

Wind loadings for the 30-story and 40-story buildings were acquired from TPU wind tunnel testing data for wind tunnel cases with the closest aspect ratios in the suburban terrain. The steps to convert the measured pressures to wind loading applicable to finite element model are also explained in this chapter. 


\subsection{Experimental Procedure}

\subsubsection{Wind Test Facility and Characteristics of Approaching Flow}

Wind pressure experiments were carried out in the 12-fan Wall of Wind (WOW) facility at Florida International University. It is an open jet facility with the capability of testing of structures in hurricane-level wind speeds $(70 \mathrm{~m} / \mathrm{s})$, Figure $5-1$. Electric fans are arranged in two rows of six fans generating a wind field with $20 \mathrm{ft}$. $(6 \mathrm{~m})$ width and $14 \mathrm{ft}$. $(4.3 \mathrm{~m})$ height. The contraction section attains a uniform flow field and is followed by a $32 \mathrm{ft}$. $(9.75 \mathrm{~m})$ long flow conditioning section containing spires and floor roughness, which generates a simulation of the Atmospheric Boundary Layer (ABL), including turbulence characteristics of the desired flow. The model was located on a turntable $20 \mathrm{ft}$. $(6.1 \mathrm{~m})$ from the exit of the flow conditioning section. 


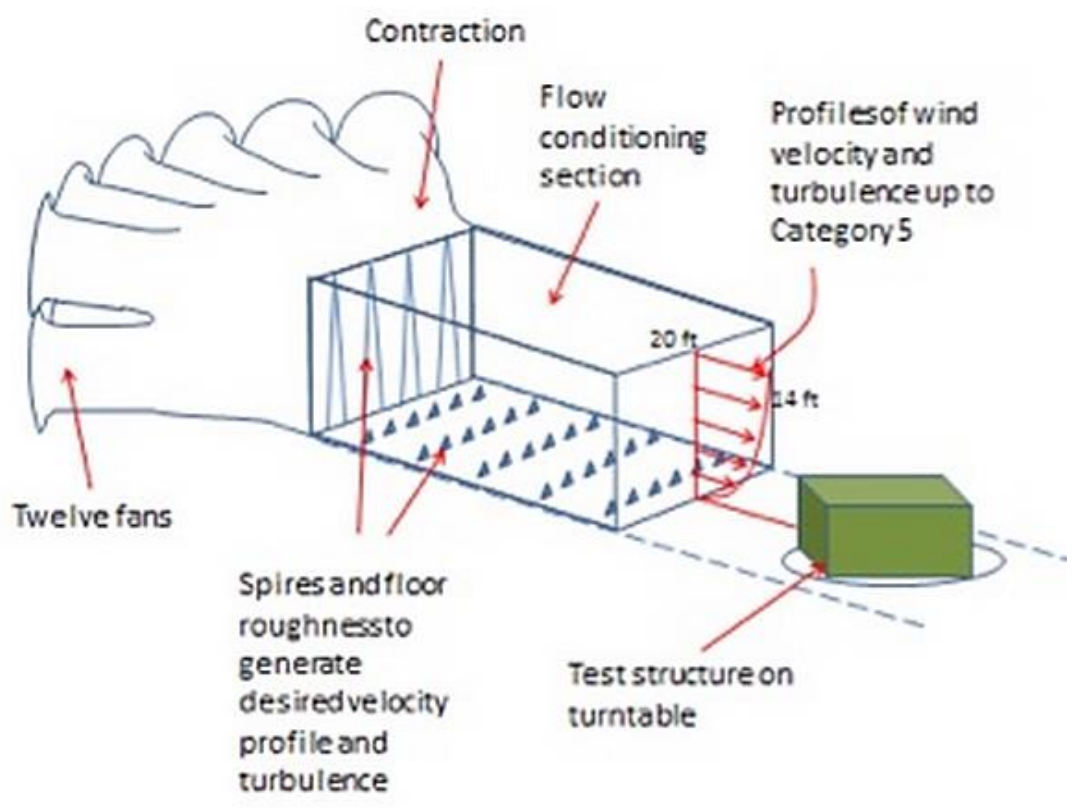

(a)

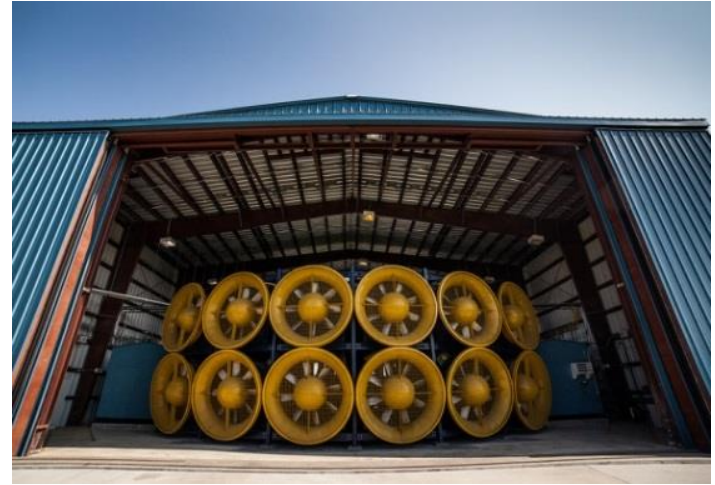

(b)

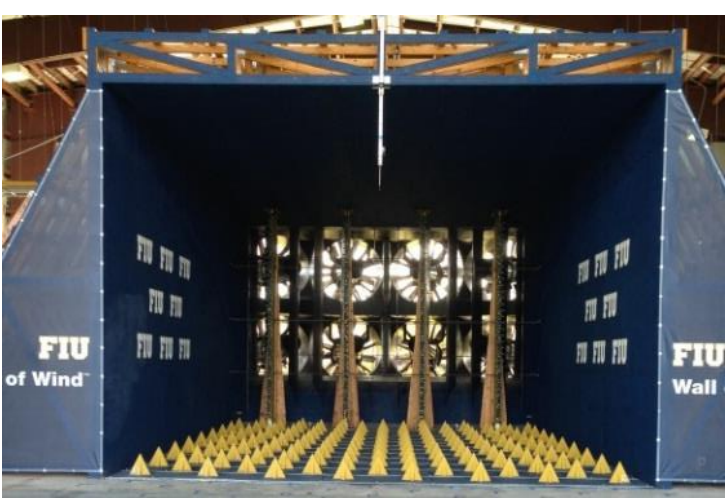

(c)

Figure 5-1. 12-fan Wall of Wind; (a) schematic model, (b) intake side, (c) exit side

In this study, a flow condition corresponding to suburban terrain was simulated using triangular spires and roughness elements. Before installing the model, measurements were carried out to evaluate the characteristics of the incoming wind. Figure 5-2a shows the mean wind speed profile versus the target $\mathrm{ABL}$ according to ASCE 7-10 specifications for exposure $\mathrm{B}$ (power law coefficient $\alpha=1 / 4)$. Figure 5-2b shows the turbulence intensity of wind flow compared with ASCE 7-10 specifications for exposure B and AIJ Recommendations (AIJ, 2004) for Categories IV and V. Although the mean wind speed profile is comparable to the code, the longitudinal turbulence 
intensity profile is lower than the value suggested by ASCE 7 but higher than AIJ-IV (AIJ 2004). Generally, the lower longitudinal turbulence intensity may result in overestimates of the acrosswind and torsional responses as well as underestimates of the along-wind responses (Kwon, Spence, \& Kareem, 2014)

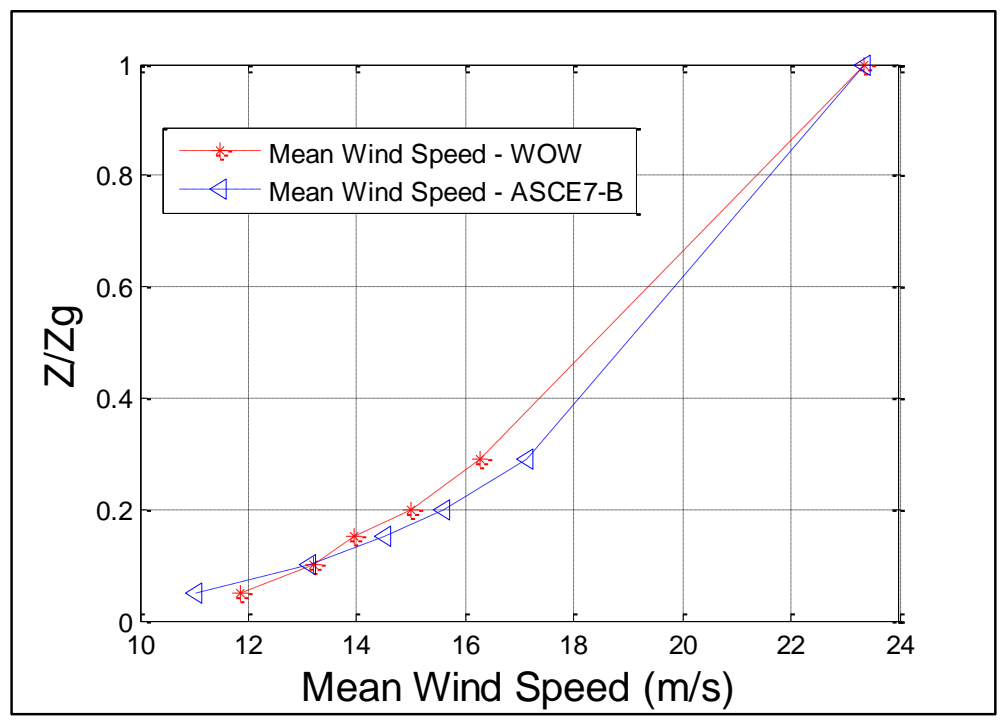

(a)

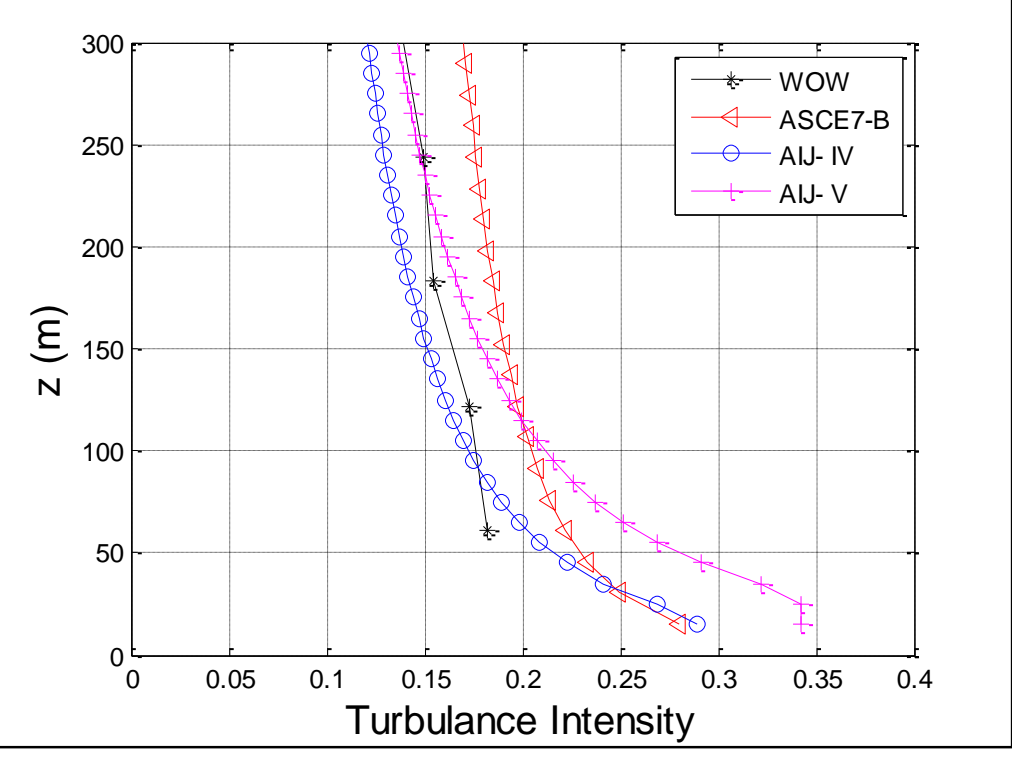

(b)

Figure 5-2. Simulated suburban terrain: (a) ABL profile and (b) turbulence intensity profile 


\subsubsection{Test Models}

To study wind loads on the 47-story building, a rigid model with a geometric length scale of 1:400 was made from 1/8-inch-thick Plexiglas sheets. Three sets of wind tests, corresponding to three levels of comprehensiveness, were carried out to evaluate the significance associated with ignoring the realistic environmental detail in each level. The three sets corresponded: (1) Test \#1 tests on an isolated prismatic shape model, Figure 5-3a; (2) Test \#2 - isolated full building model (including the penthouse and the skirt), Figure 5-3b; and (3) Test \#3 - full building model and all existing buildings within a full-scale radius of approximately $650 \mathrm{ft}$. (200 m) from the 47-story building at the time of Hurricane Alicia, 1983, Figure 5-3c.

Figure 5-4 illustrates the pressure tap distribution on the model surfaces and the roof of the prismatic model. As shown in the figure, 90 pressure taps on each face of the model in addition to 24 pressure taps on the roof, totaling 384 pressure taps were uniformly mounted on the model to permit measurements of the pressure distribution during the wind tests. For the second set of tests the penthouse and the skirt, with the detail (shown in Figure 5-4), were added to the prismatic model. Finally, the third set of the tests were carried out after mounting immediate surrounding buildings (see Figure 5-5). 


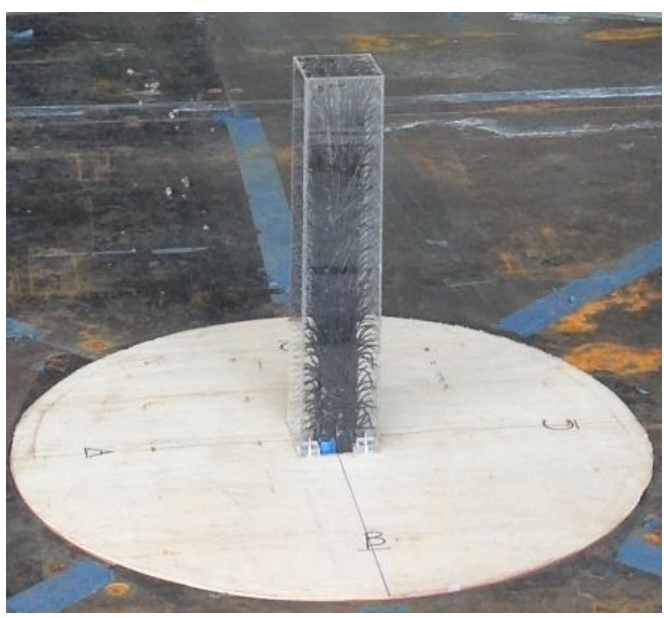

(a)

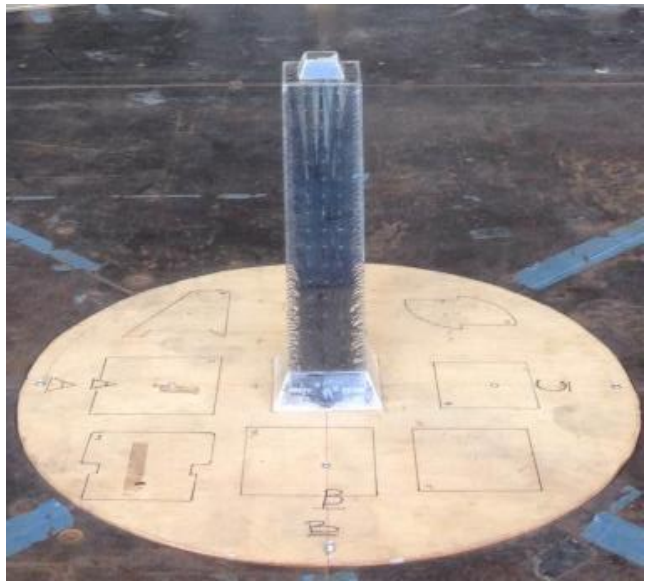

(b)

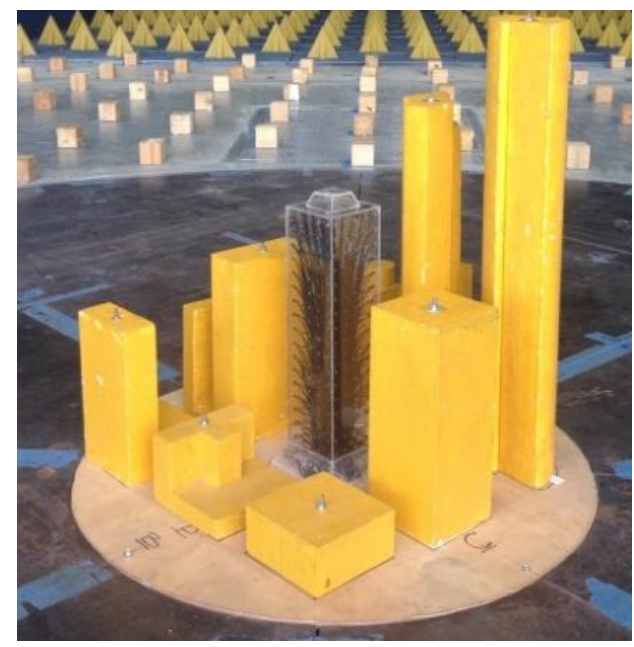

(c)

Figure 5-3. Test Models: (a) Test \#1: isolated prismatic shape model, (b) Test \#2: isolated full building model, (c) Test \#3: full building model and surrounding buildings 

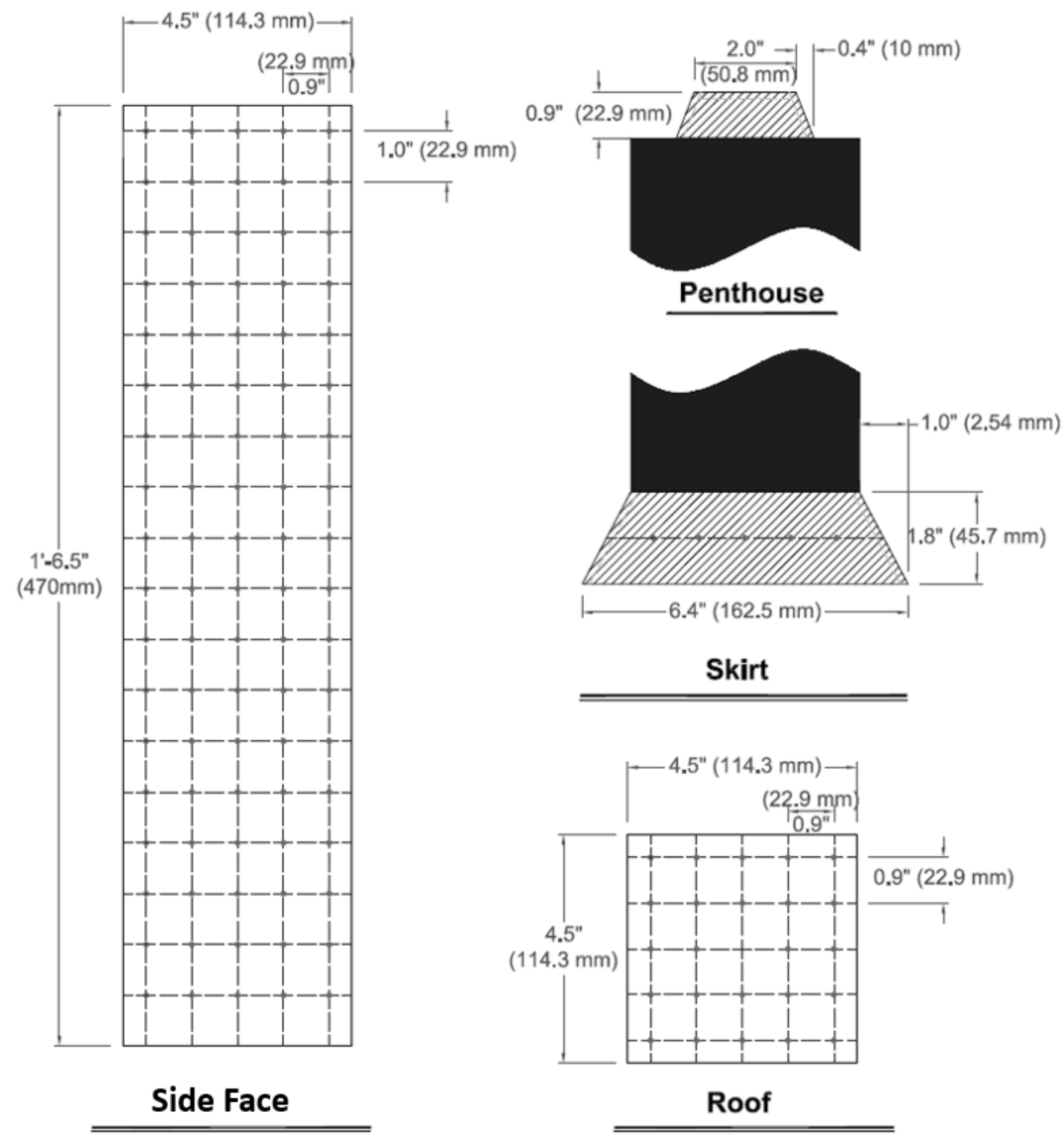

Skirt

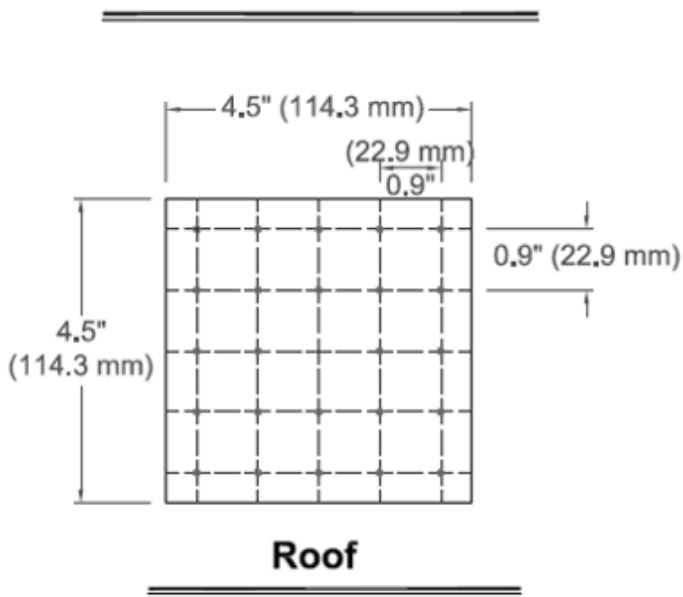

Figure 5-4. Wind tunnel model details including pressure taps layout on the sides and the roof of the prism-shape model; scaled detail of the penthouse; and scaled detail of the skirt part 


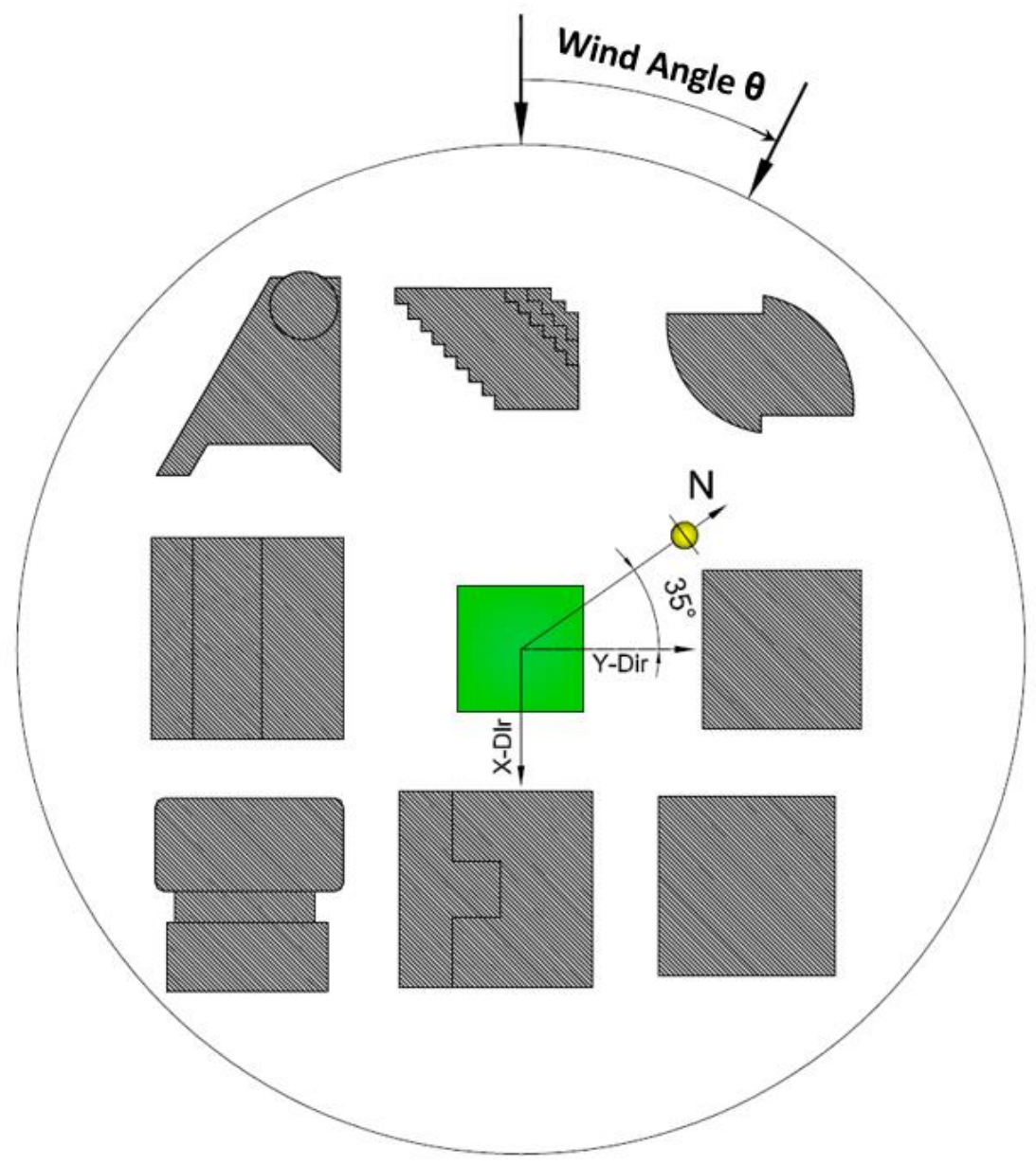

Figure 5-5. Immediate surroundings of the 47-story building, illustration of approaching wind and the notation of the directions

In these experiments, six 64-channel Scanivalve Corporation pressure scanning systems were used for pressure measurements. Each set of the wind tests was done by conducting the pressure measurements for incrementally increasing wind angles as shown in Table 5-1. The wind angle $\theta$ was defined as the angle from the given X-Dir axis (see Figure 5-5 for the coordinates and force notations) along a clockwise direction. In each single test, pressure data were acquired at a sampling frequency of $512 \mathrm{~Hz}$ for a period of one minute. During the wind tests the wind speed was measured at 10 feet $(3.05 \mathrm{~m})$ height using a Cobra probe mounted on a portal frame over the model. 
Table 5-1. Protocol of the wind pressure tests

\begin{tabular}{|c|c|c|c|c|c|c|c|c|c|c|}
\hline \multicolumn{11}{|c|}{ Testing Protocol for High-rise Building Model Tests } \\
\hline \multirow{3}{*}{ Test_\#1 } & \multirow{2}{*}{$\begin{array}{c}\text { Wind } \\
\text { Angles } \\
\text { (degree) }\end{array}$} & 0 & 5 & 10 & 15 & 20 & 25 & 30 & 35 & 40 \\
\hline & & 45 & 50 & 55 & 60 & & & & & \\
\hline & \multicolumn{3}{|c|}{ Exposure $=\mathrm{B}$} & \multicolumn{5}{|c|}{$\begin{array}{c}\text { Mean reference wind speed }=30 \mathrm{mph}(13.4 \mathrm{~m} / \mathrm{s}) \text { at } \\
\text { roof top }\end{array}$} & \multicolumn{2}{|c|}{$\begin{array}{l}\text { Duration= } \\
1 \text { min }\end{array}$} \\
\hline \multirow{3}{*}{ Test_\#2 } & \multirow{2}{*}{$\begin{array}{l}\text { Wind } \\
\text { Angles } \\
\text { (degree) }\end{array}$} & 0 & 5 & 10 & 15 & 20 & 25 & 30 & 35 & 40 \\
\hline & & 45 & 50 & 55 & 60 & & & & & \\
\hline & \multicolumn{3}{|c|}{ Exposure $=\mathrm{B}$} & \multicolumn{5}{|c|}{$\begin{array}{c}\text { Mean reference wind speed }=30 \mathrm{mph}(13.4 \mathrm{~m} / \mathrm{s}) \text { at } \\
\text { roof top }\end{array}$} & \multicolumn{2}{|c|}{$\begin{array}{l}\text { Duration= } \\
1 \text { min }\end{array}$} \\
\hline \multirow{4}{*}{ Test_\#3 } & \multirow{3}{*}{$\begin{array}{l}\text { Wind } \\
\text { Angles } \\
\text { (degree) }\end{array}$} & 0 & 15 & 30 & 45 & 60 & 75 & 90 & 105 & 120 \\
\hline & & 135 & 150 & 165 & 180 & 195 & 210 & 225 & 240 & 255 \\
\hline & & 270 & 285 & 300 & 315 & 345 & & & & \\
\hline & \multicolumn{3}{|c|}{ Exposure $=\mathrm{B}$} & \multicolumn{5}{|c|}{$\begin{array}{l}\text { Mean reference wind speed }=30 \mathrm{mph}(13.4 \mathrm{~m} / \mathrm{s}) \text { at } \\
\text { roof top }\end{array}$} & \multicolumn{2}{|c|}{$\begin{array}{l}\text { Duration= } \\
1 \text { min }\end{array}$} \\
\hline
\end{tabular}

For the pressure measurements 4-foot $(1.22 \mathrm{~m})$ long, 0.053-in $(1.34-\mathrm{mm})$ diameter PVC tubes were used to connect the pressure taps to the pressure scanners. As shown in Figure 5-6, the pressure tubing transfer function (Irwin, Cooper, \& Girard, 1979) was measured for the given tubing system to account for possible pressure signal distortion. Finally, the data collected were low-pass filtered with a cutoff frequency of $120 \mathrm{~Hz}$. 


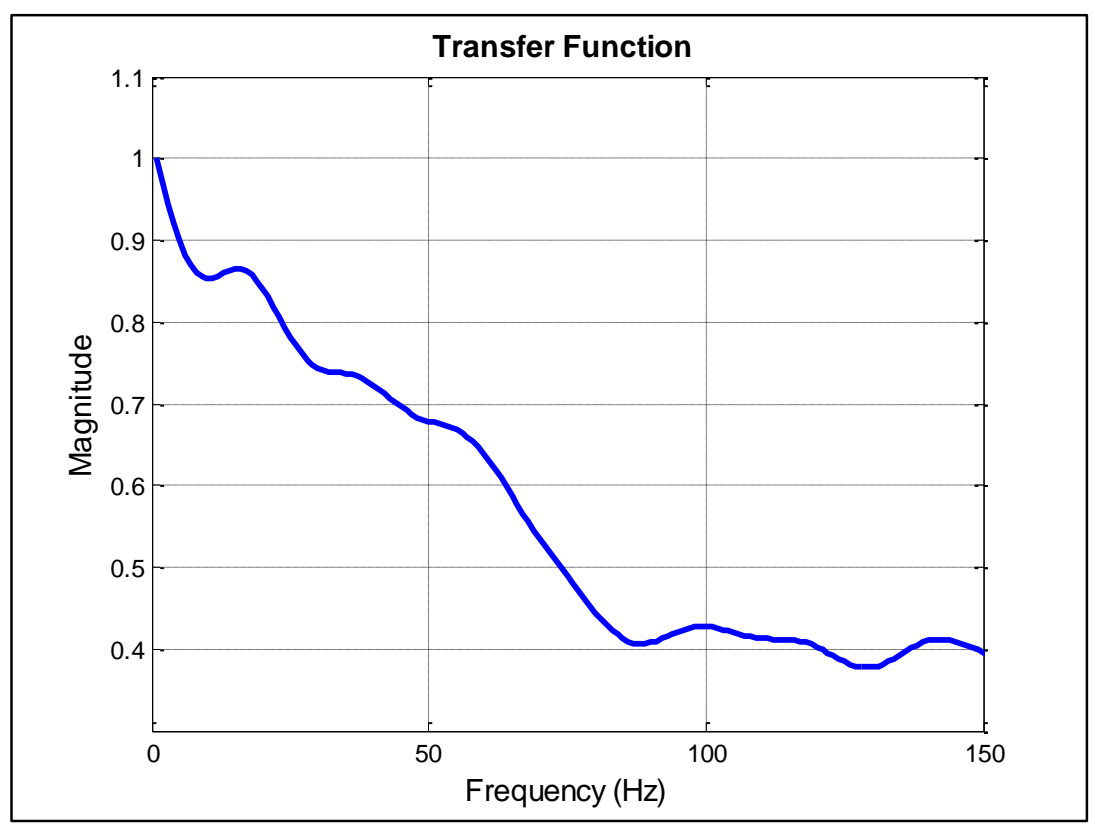

(a)

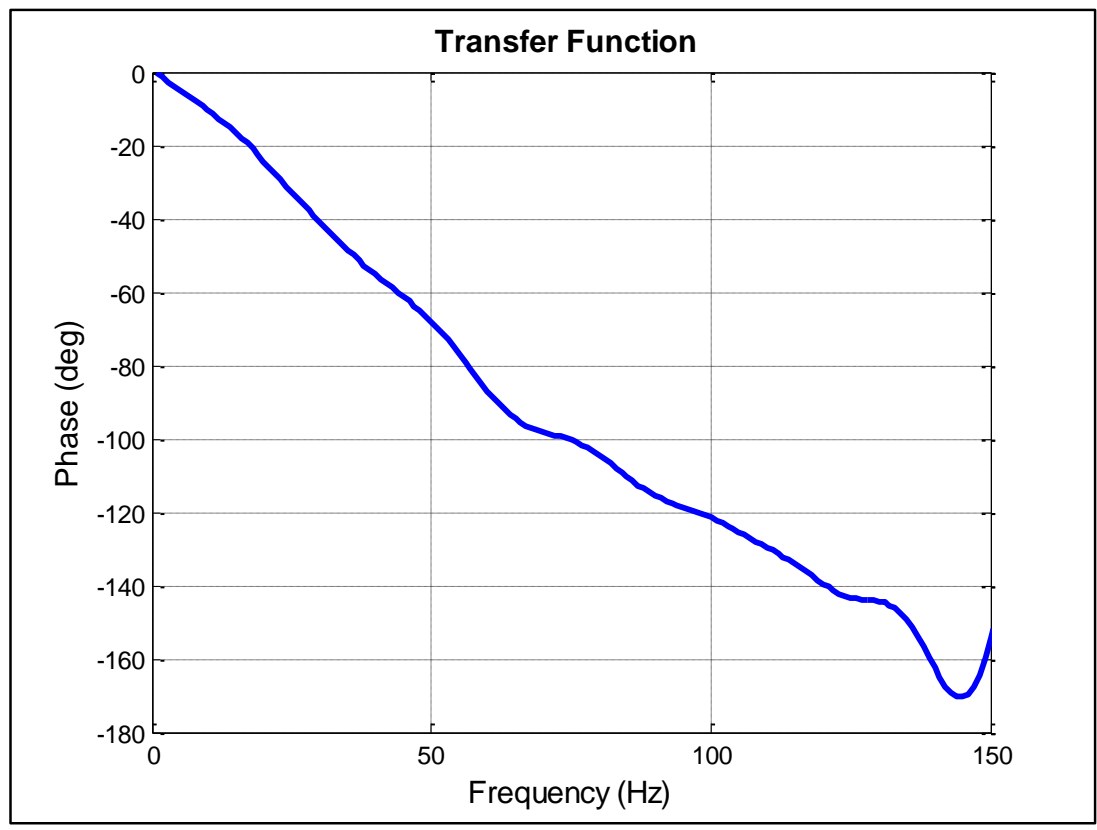

(b)

Figure 5-6. Transfer function of the tubing system 


\subsubsection{Definition of Studied Parameters}

The distribution of aerodynamic wind pressure on the model is presented in terms of mean pressure coefficient contours over the faces around the building model. The mean pressure coefficient for each pressure tap is expressed as follows:

$\overline{C_{p_{\iota}}}=\frac{\bar{P}_{l}}{P_{\text {ref }}}=\frac{\bar{P}_{l}}{\frac{1}{2} \rho V_{\text {ref }}{ }^{2}}$

Where $\bar{P}$ is the average measured pressure at tap $i ; P_{\text {ref }}$ is the reference wind pressure of the of the approaching wind at roof height. $V_{r e f}$ is mean velocity at reference height (roof height); and $\rho$ is the air density.

The aerodynamic forces acting on the high-rise building model are presented in terms of mean base force coefficients and fluctuating base force coefficients with incident wind direction as a variable. Mean base force coefficients $\overline{C_{F x}}$ and $\overline{C_{F y}}$ are expressed as follows:

$\overline{C_{F x}}=\frac{\bar{F}_{x}}{\frac{1}{2} \rho V_{r e f}{ }^{2} B H}$
$\overline{C_{F y}}=\frac{\overline{F_{y}}}{\frac{1}{2} \rho V_{r e f}{ }^{2} B H}$

Where $\bar{F}_{x}$ and $\bar{F}_{x}$ are average base shear in $\mathrm{x}$ and y direction respectively, and $H$ and $B$ are the height and width of the building respectively. The fluctuating base force coefficients $C_{F x_{r m s}}$ and $C_{F y_{r m s}}$ are expressed as following:

$\begin{aligned} C_{F x_{r m s}} & =\frac{\sigma_{F x}}{\frac{1}{2} \rho V_{r e f}^{2} B H} \\ C_{F y_{r m s}} & =\frac{\sigma_{F y}}{\frac{1}{2} \rho V_{r e f}{ }^{2} B H}\end{aligned}$ 
Where $\sigma_{F x}$ and $\sigma_{F y}$ are standard deviation of base shear forces in the $\mathrm{x}$ and $\mathrm{y}$ direction respectively. Area averaged wind pressure coefficients on wall surfaces of model were used in this study to provide a comparison between the two set of wind test data obtained in this study and wind tunnel data presented in TPU aerodynamic database. The area averaged wind pressure coefficient for face $j$ and time $t$ was obtained using following equation.

Area Average $C_{p}(j, t)=\frac{\sum_{i=1}^{N j} C_{p_{i}}(t) \cdot A_{i}}{\sum_{i=1}^{N j} A_{i}}$

Where $C_{p_{i}}(t)$ is the pressure coefficient at time $t$ for the pressure tap at point $i ; A_{i}$ is the effective area of wind pressure measured at point $i$; and $N_{j}$ is the number of measured points on surface $j$.

\subsubsection{Wind Test Results and Discussion}

The mean wind pressure coefficient distribution on the surfaces of the model for Test_\#1 (isolated prismatic model) and Test \#2 (isolated full building model) are presented for the wind at the zero-degree direction in Figure 5-8 and Figure 5-8 respectively. The figure shows the pressure coefficients on the models when the wind flow is normal to the upstream face. As expected, in both tests the mean pressure coefficients are generally positive on the windward wall with a maximum value at the stagnation point approximately two-thirds of the way up the height of the building. But in Test_\#2 mean pressure coefficients at the skirt abruptly decrease, leading to negative pressures (suction) in lower elevations close to the base. Due to flow separation at the edges of the windward face, negative pressures (suction) are generally observed on the side walls and leeward face. For Test_\#2 at the skirt, the mean pressure coefficients tend to increase on the side walls and leeward face. This change is more significant on the leeward face. Comparing the pressure distribution in Test_\#1 and Test_\#2 indicates that building shape characteristics of the 47-story building, particularly at the skirt, affected the local pressure distribution, which would be 
relevant in the design of the system cladding. However, apart from this change overall wind pressure magnitude and distribution was not significantly affected.

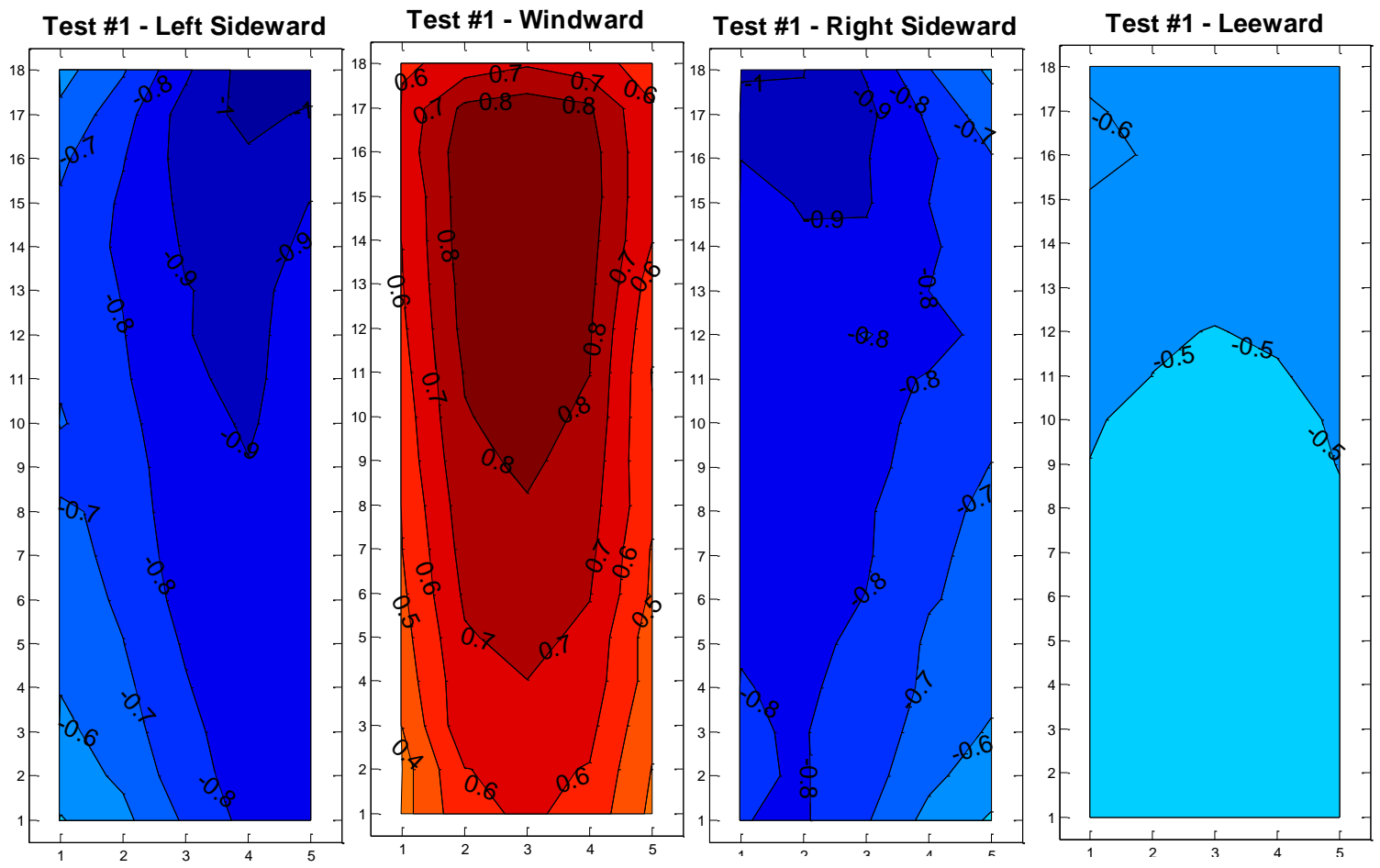

Figure 5-7. Surface mean pressure coefficient distribution for Test \#1 (prismatic shape model) for the wind flow normal to the upstream face 

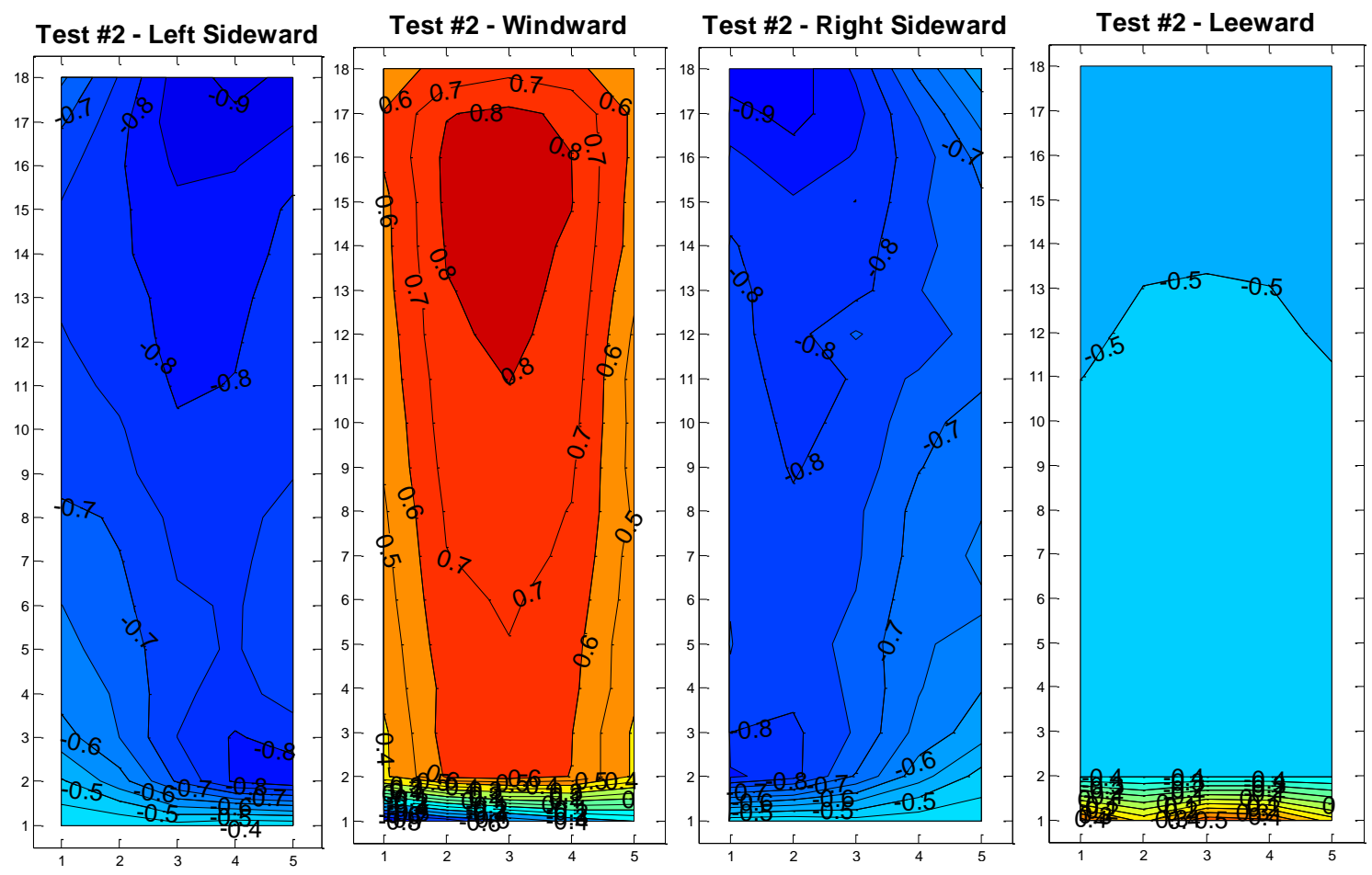

Figure 5-8. Surface mean pressure coefficient distribution for Test \#2 (including penthouse and the skirt) for the wind flow normal to the upstream face

Figure 5-9 presents the mean base force coefficients, $\overline{C_{F x}}$ and $\overline{C_{F y}}$ respectively, in the noted $\mathrm{X}$ and $\mathrm{Y}$ directions of the building as a function of wind direction. Because of biaxial symmetry, data obtained for directions $0^{\circ}$ to $60^{\circ}$ could be used to extend results to cover the full $360^{\circ}$ range of wind direction in Test \#1 and \#2. As shown in the figure, the mean wind force coefficient for the isolated model, Test \#1 and Test \#2, are almost coincident for most wind directions, i.e. adding the penthouse and skirt did not affect the average wind forces significantly. However, a slight decrease in average force coefficients can be seen for wind directions where the magnitude of the force coefficient was highest. The figure indicates that the results from Test \#3, where the model included the surrounding buildings, departed very significantly from the other results. The interference effects of surrounding building generally lead to large reductions in mean wind forces on the 47-story building. 


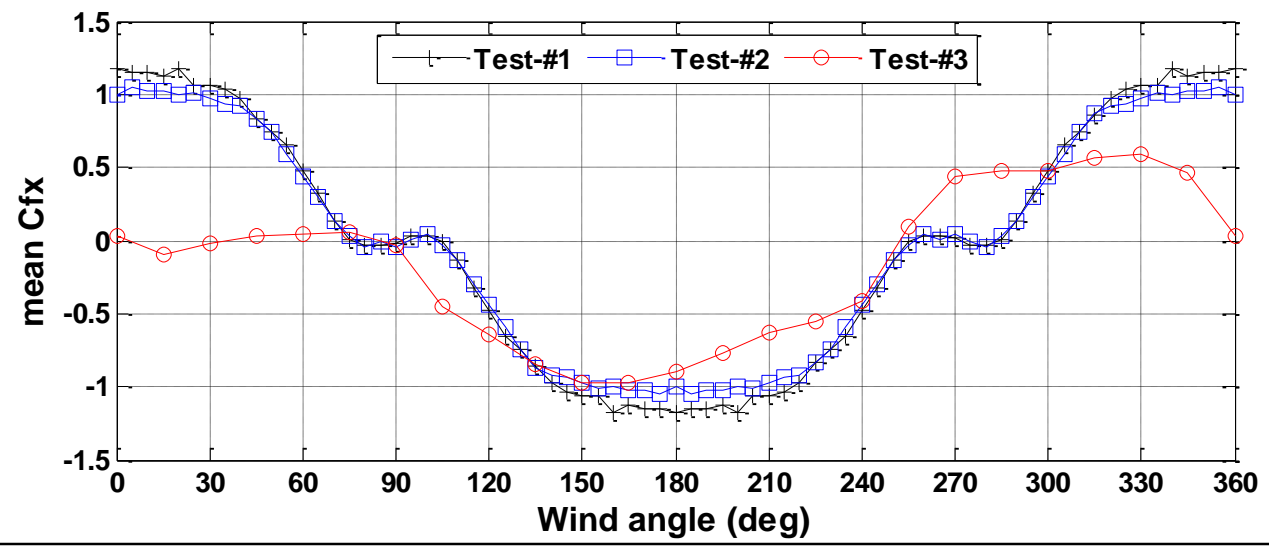

(a)

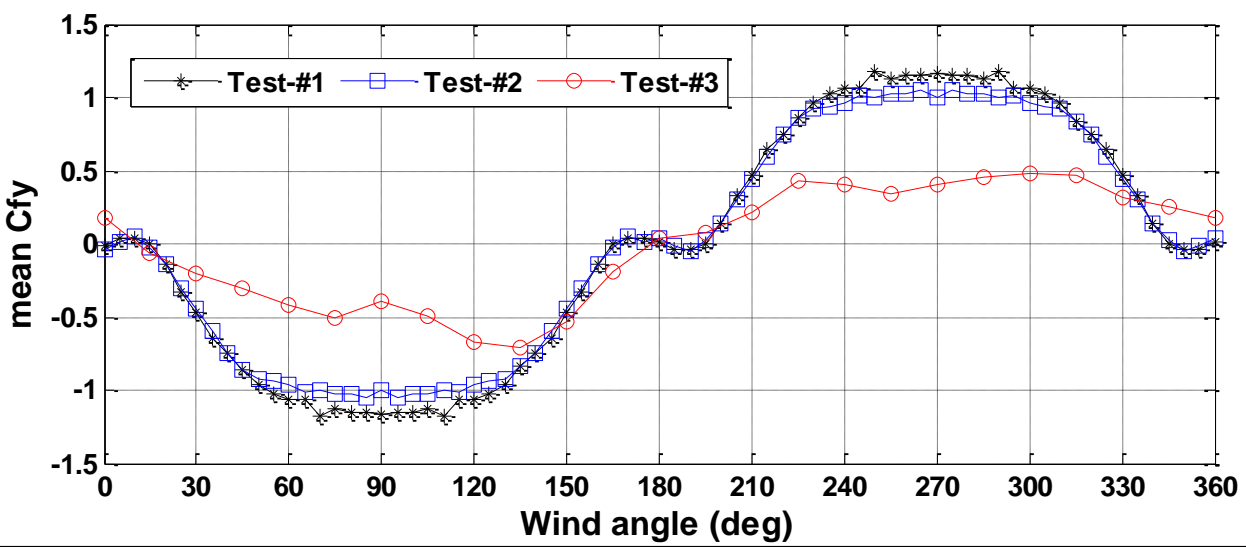

(b)

Figure 5-9. Mean base force coefficients versus the wind directions: $(a)$ in the $x$-direction; $(b)$ in the $y$-direction

The fluctuating base force coefficients in the $\mathrm{X}$ and $\mathrm{Y}$ directions, $C_{F x_{r m s}}$ and $C_{F y_{r m s}}$, are shown in Figure 5-10 as a function of wind direction. The figure indicates that the presence of the penthouse and the skirt slightly decreased the fluctuating wind forces on 47-story building model. The model surrounded by neighboring buildings experienced very significant reductions in fluctuating wind forces for most wind directions. The behavior of the mean and fluctuating base force coefficients of Test \#3 shown in Figure 5-10 suggest that the directions from $120^{\circ}$ to $180^{\circ}$ where the building gets the least sheltering from surrounding buildings constitute the critical wind directions. 


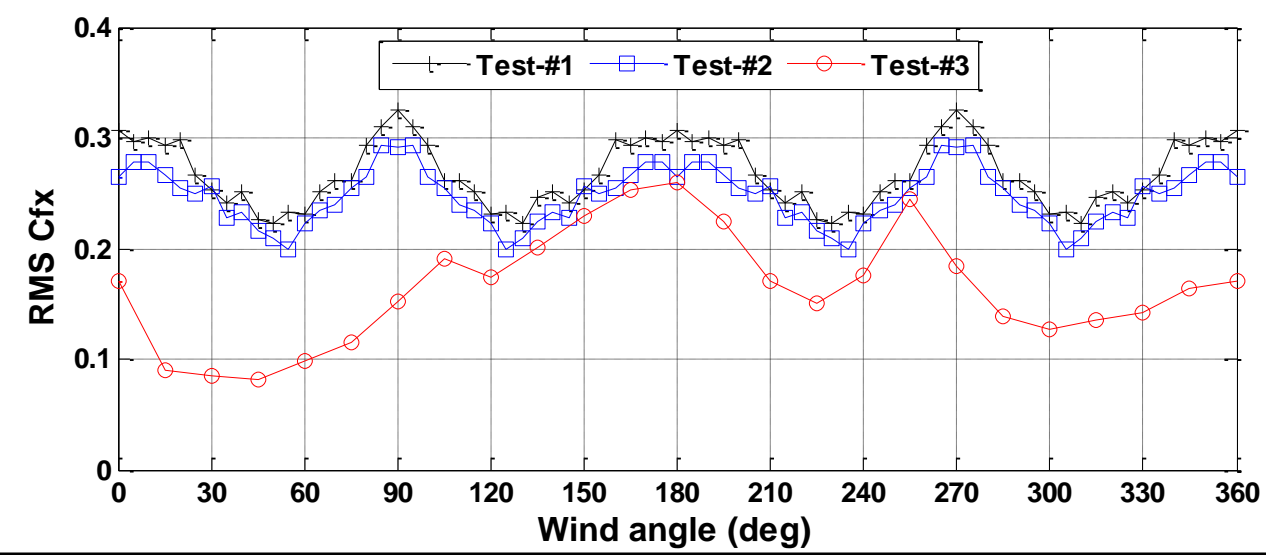

(a)

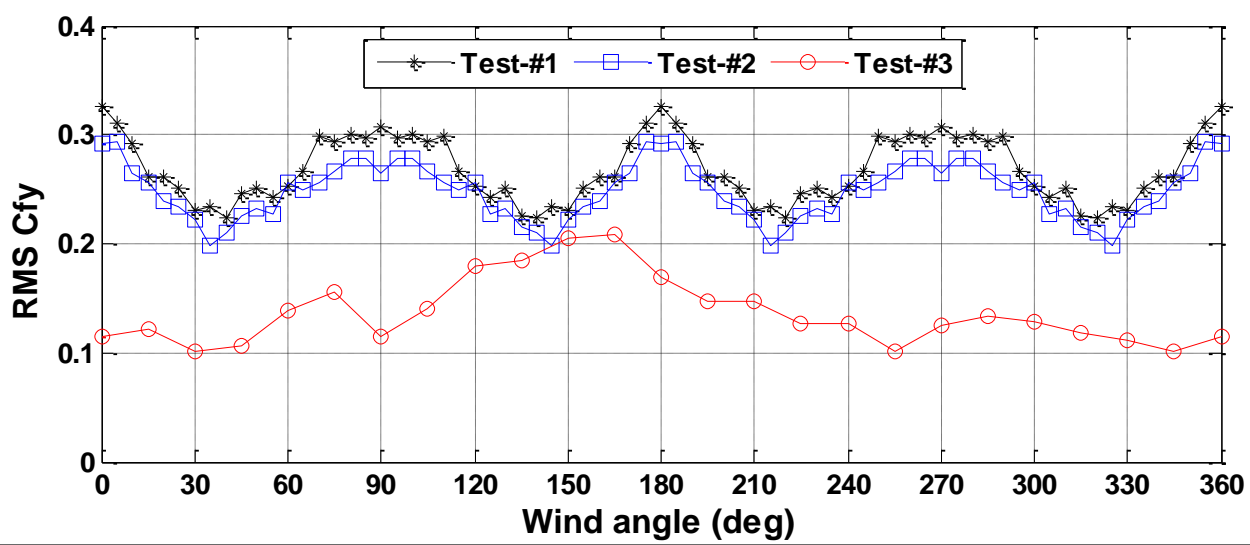

(b)

Figure 5-10. Fluctuating base force coefficients versus the wind directions: (a) in the $x$-direction; (b) in the $y$-direction

\subsubsection{Comparing Results with TPU Wind Tunnel Database}

Tokyo Polytechnic University (TPU) has provided a web-based database on wind tunnel experiments of high-rise buildings with varied aspect ratios, entitled: "TPU Aerodynamic database of high-rise buildings." The database provides wind tunnel data such as pressure coefficient time series and statistical values of local wind pressure coefficients for high-rise buildings for a range of Breadth/Depth/Height ratios. For the 47-story building with approximate Breadth/Depth/Height ratio equal to $1 / 1 / 4$, the TPU wind tunnel data was acquired. The corresponding TPU wind tunnel data is related to a 3.9 in $\times 3.9$ in $\times 15.7$ in $(0.1 \mathrm{~m} \times 0.1 \mathrm{~m} \times 0.4 \mathrm{~m})$ 
model with 400 mounted pressure taps (100 pressure taps on each wall). Wind pressure measurements were made at a sampling frequency of $1000 \mathrm{~Hz}$ and with average wind speed at roof elevation around $24.6 \mathrm{mph}(11 \mathrm{~m} / \mathrm{s})$.

The TPU wind tunnel results were compared with WOW test result for the isolated prismatic model (Test-\#1) to assess the level of consistency of the wind tunnel test results. Figure 5-11 shows area averaged wind pressure coefficients on wall surfaces of the building model obtained from both the TPU wind tunnel data and WOW test results. The figure indicates that the agreement between the TPU and WOW results is reasonable. The differences are within the range that can be attributed to the differences in the modeling of the incident turbulence and terrain characteristics. 


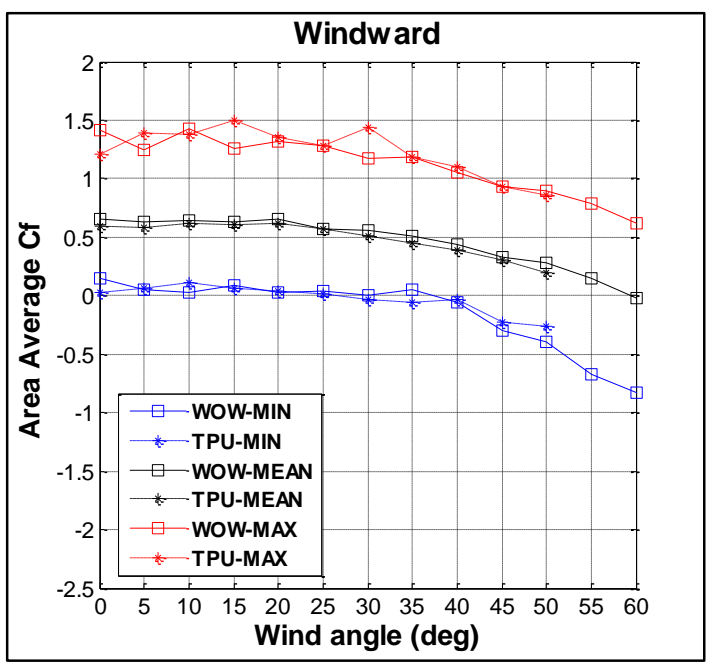

(a)

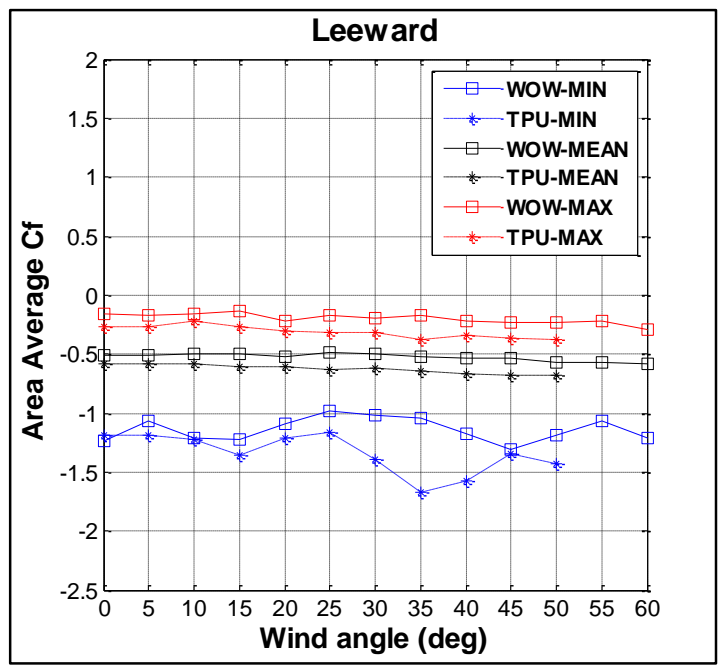

(c)

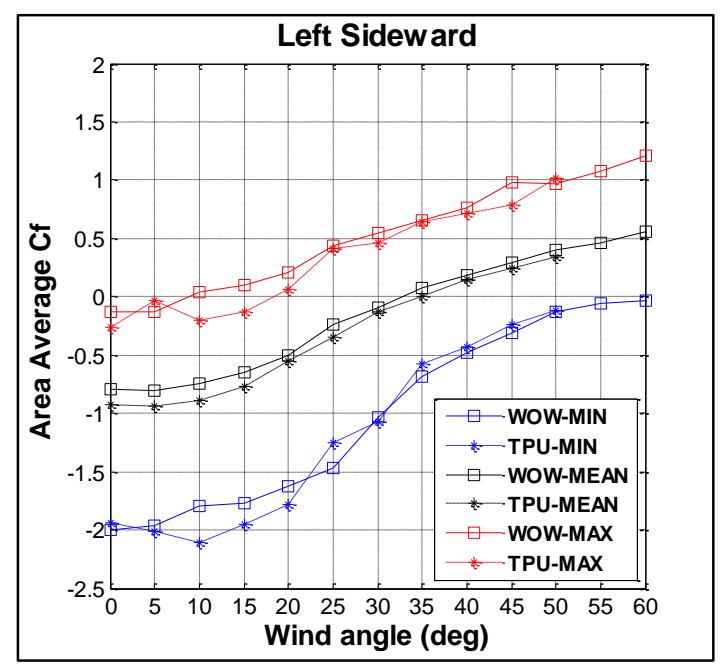

(b)

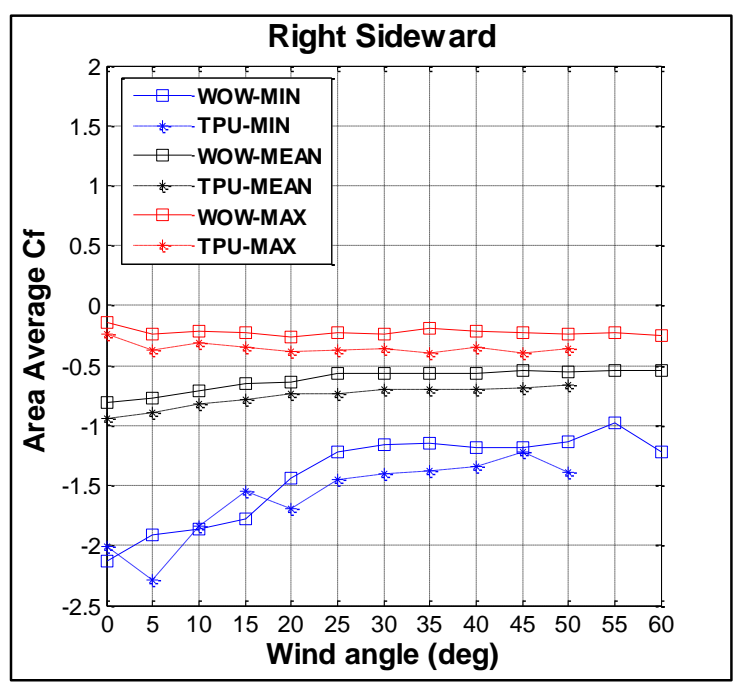

(d)

Figure 5-11. Area average $C_{f}$ of WOW and TPU results for testing the isolated model: (a) on the windward face; (b) on the left sideward face; (c) on the leeward face; and (d) on the right sideward face

\subsection{Wind Time-History Loading}

As described in the previous section the wind loading for the 47-story model was measured by conducting wind pressure test in WOW facility. The following section provides an introduction to TPU database which is used to acquire wind loading data for two other buildings. Then, the procedure to produce wind loading applicable to finite element model from either WOW or TPU wind data is explained. 


\subsubsection{TPU Wind Tunnel Database}

TPU Aerodynamic Database for High-rise Buildings provides wind tunnel test data of wind loads on high-rise buildings. It also contains contours of statistical values of local wind pressure coefficients, graphs of statistical values of area averaged wind pressure coefficients on the wall surfaces and time series data of point wind pressure coefficients. The data corresponding to models with different aspect ratios in different wind directions is presented on the website. The database can be used to calculate local wind pressures, area averaged wind pressure coefficient on wall surfaces, and wind-induced dynamic responses of high-rise buildings. Figure 6-2 shows a typical data page on TPU website indicating various types of raw time history data and corresponding available statistical outputs.

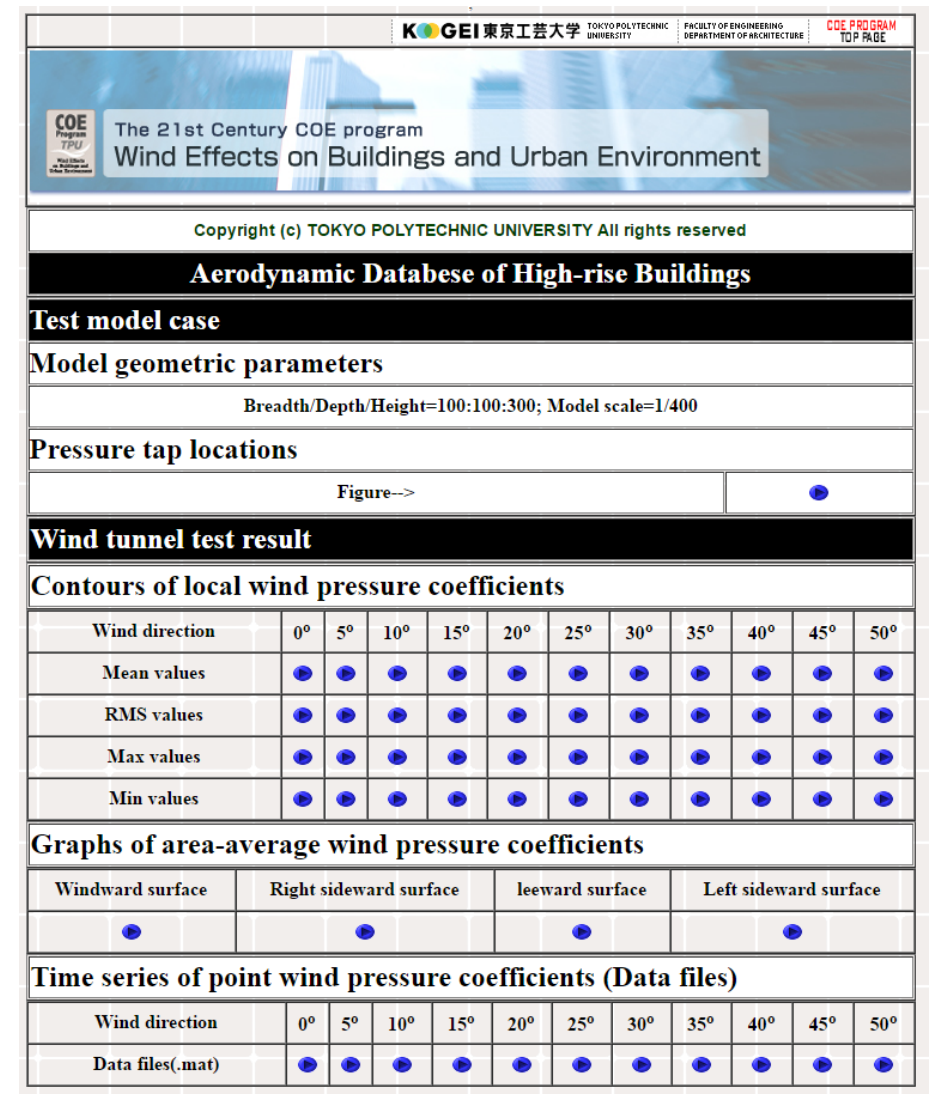

Figure 5-12. Typical data available on TPU Aerodynamic Database (http://wind.arch.tkougei.ac.jp/system/eng/contents/code/tpu) 
The database provides wind pressure coefficients time series for a grid of pressure channels around the model measured during wind tunnel testing. Figure 5-13a shows a typical channel positioning provided by the TPU database and the corresponding positions around the building. The arrangement of pressure taps in respect to their location on different faces around the building is illustrated in Figure 5-13b. 


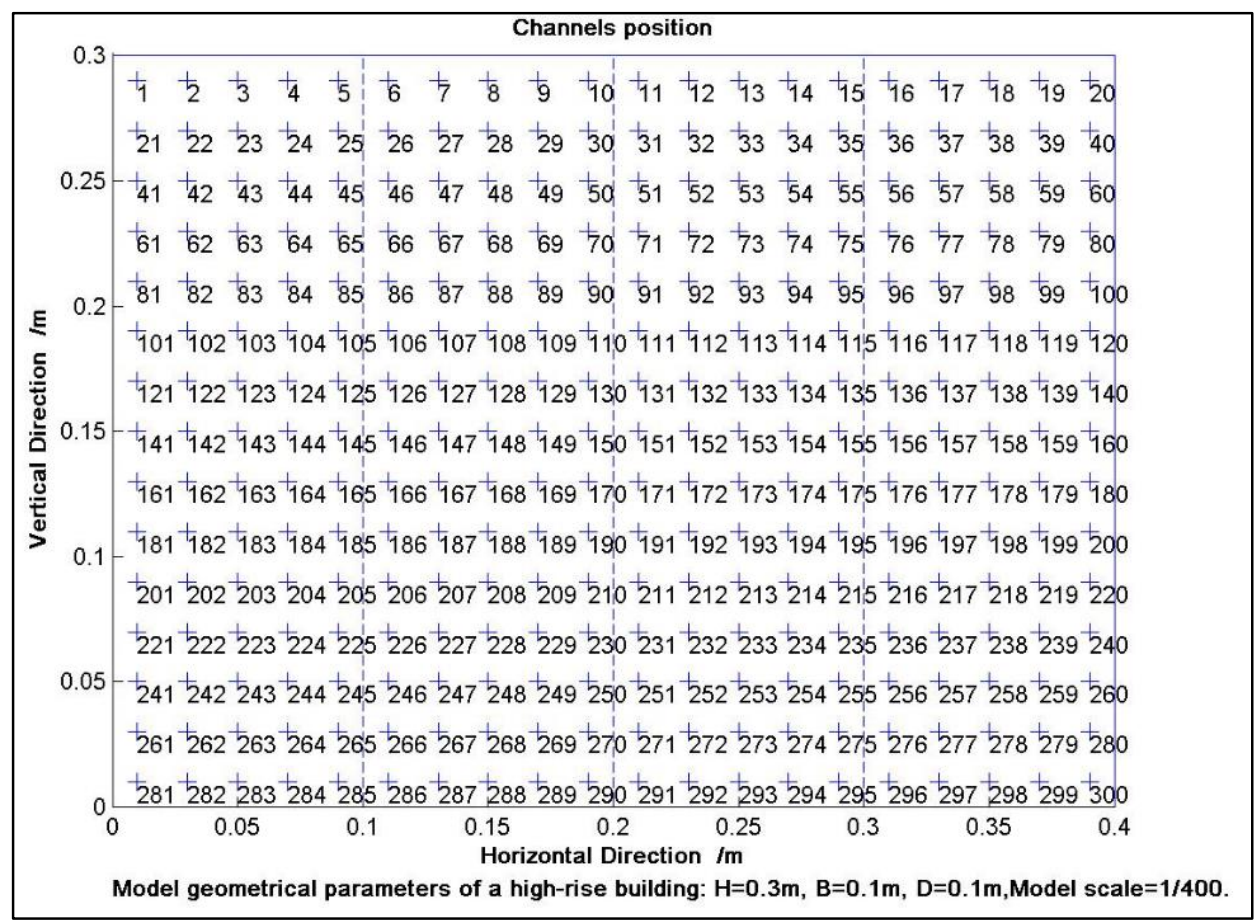

(a)

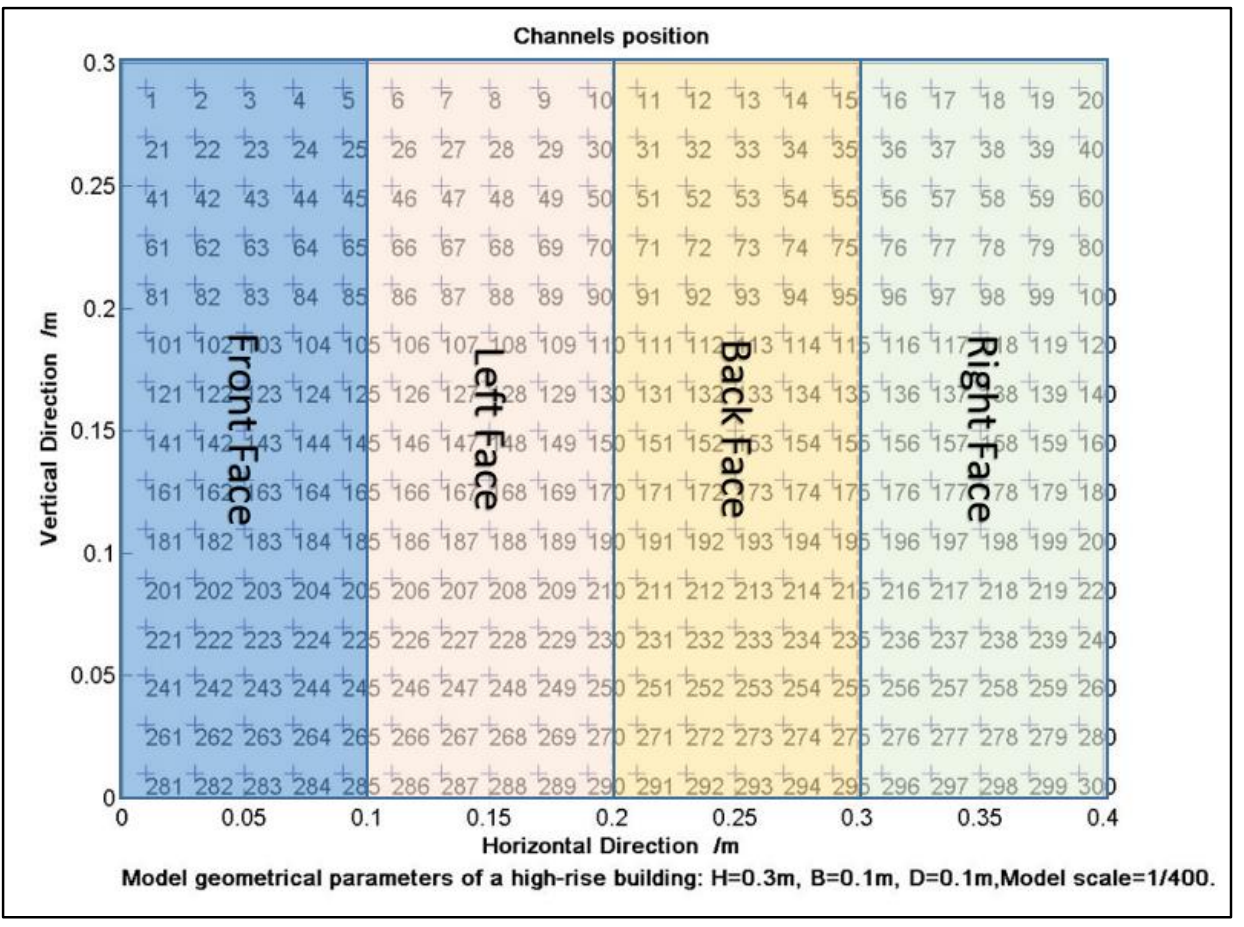

(b)

Figure 5-13. Typical pressure channel positioning in TPU Aerodynamic Database and corresponding positions around the building (http://wind.arch.tkougei.ac.jp/system/eng/contents/code/tpu) 
The database provides wind tunnel data for building cases with specific aspect ratios of breadth, depth, and height of the building (as shown in Figure 5-14). For 30-story building with actual aspect ratios: $\mathrm{B} / \mathrm{D}=1: 1$ and $\mathrm{B} / \mathrm{H}=1: 2.63$ ( $\mathrm{B}$ : breadth, $\mathrm{D}$ : depth and $\mathrm{H}$ : height), and for 40-stroy building with actual aspect ratios: $\mathrm{B} / \mathrm{D}=1: 1$ and $\mathrm{B} / \mathrm{H}=1: 4.375$ the available data for models with closest aspect ratios $(\mathrm{B} / \mathrm{D}=1: 1$ and $\mathrm{B} / \mathrm{H}=1: 3$ and $\mathrm{B} / \mathrm{D}=1: 1$ and $\mathrm{B} / \mathrm{H}=1: 4$ respectively) were obtained from the database. Wind pressure coefficients related to exposure B in different wind directions from zero to $45^{\circ}$ were acquired and used to generate the corresponding dynamic wind loading.

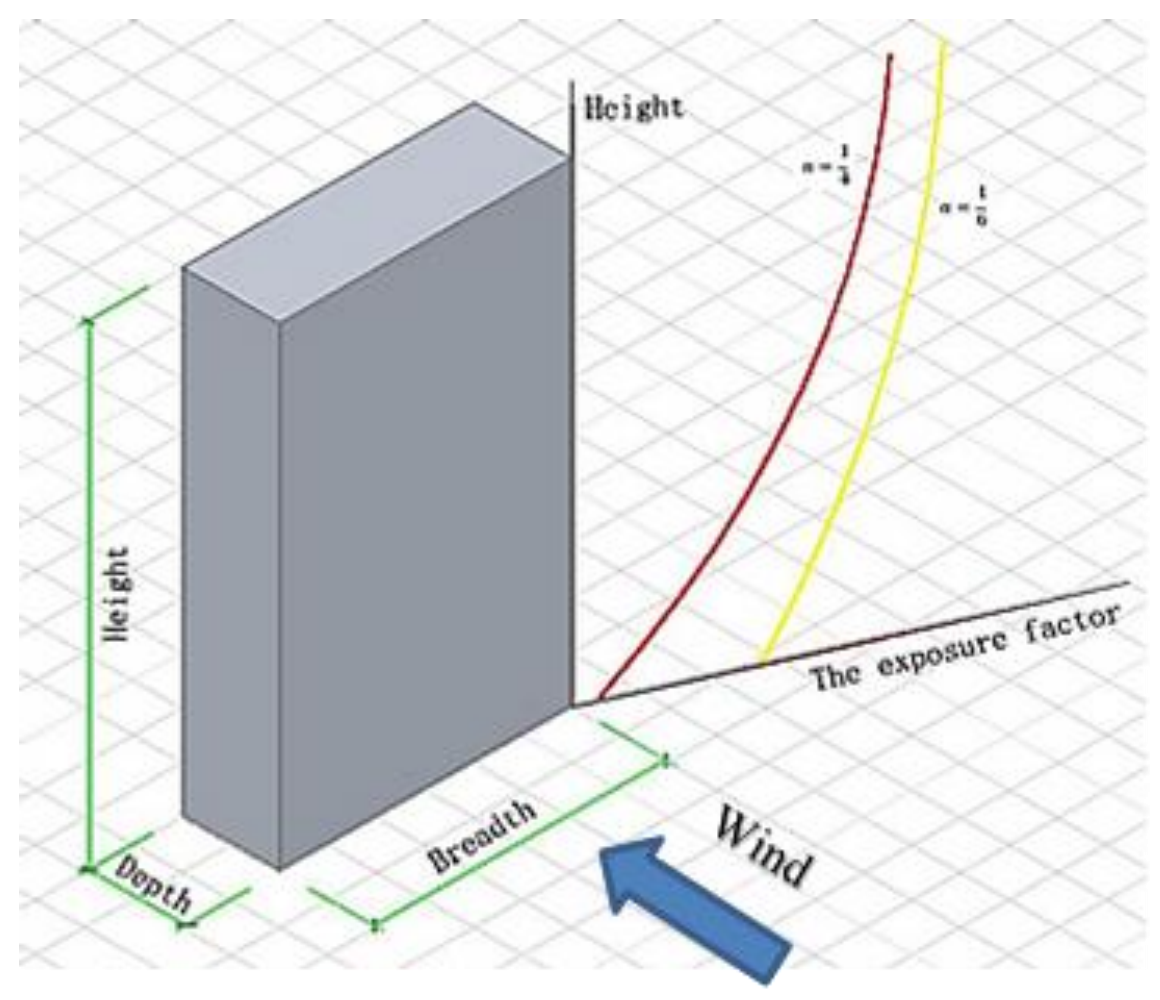

Figure 5-14. Illustration of breadth, depth and height of the building (http://wind.arch.tkougei.ac.jp/system/eng/contents/code/tpu)

\subsubsection{Wind Speed Scaling and Time Scaling}

From all above steps, the wind pressure data for three buildings from either WOW or TPU were acquired in term of pressure coefficient time series. In order to determine the equivalent pressure 
on building surface it had to be multiplied by reference pressure expressed in term of a function of square wind speed at the reference elevation:

$$
P_{i}=C_{p_{i} \cdot \frac{1}{2}} \cdot \rho \cdot V^{2}
$$

where:

$P_{i}=$ Pressure on surface of building corresponding to $\mathrm{i}^{\text {th }}$ pressure tap,

$C_{p_{i}}=$ Pressure coefficient related to $\mathrm{i}^{\text {th }}$ pressure tap,

$V=$ Mean wind velocity measured at the reference (roof) elevation.

In this study 3 -second basic wind speed in $33 \mathrm{ft}$. elevation for open terrain, used as design wind speed according to ASCE 7-10 was selected to represent the wind loading levels and associated wind hazard levels. Therefore, in each step the mean velocity at roof elevation needed to be estimated accordingly for a given basic wind speed. Both terrain and elevation conversions have to be conducted to obtain the hourly mean wind speed at roof elevation of the building.

To determine the equivalent time-history duration on the full-scale building, the time scale between actual building and model used in wind tunnel testing had to be determined. The time scale $\lambda_{T}$ is related to the geometric scale $\lambda_{L}$ and the velocity scale $\lambda_{V}$ as follows:

$$
\lambda_{T}=\frac{\lambda_{L}}{\lambda_{V}}
$$

the following section shows an example of wind speed conversion and calculating the loading time step for prototype building.

\section{Wind Speed conversion}

Through the following calculations the 115 basic wind speed at $33 \mathrm{ft}$ is used. The elevation is used to calculate the corresponding hourly mean wind speed at roof elevation of the 47-story building.

$\left[\bar{V}_{3 s}(33 \mathrm{ft})\right]_{c}=115 \mathrm{mph}$ 


$$
\begin{aligned}
& {\left[\bar{V}_{3 s}(33 f t)\right]_{c}=\left[\bar{V}_{h r}(33 f t)\right]_{c} \times 1.525} \\
& {\left[\bar{V}_{\mathrm{hr}}(33 f t)\right]_{c}=75.4 \mathrm{mph}} \\
& {\left[\bar{V}_{h r}(z)\right]_{B}=\left[\bar{V}_{\mathrm{hr}}(33 \mathrm{ft})\right]_{c} \times\left(\frac{Z_{r e f_{c}}}{z_{c}}\right)^{\frac{1}{\alpha_{c}}} \times\left(\frac{z_{B}}{z_{r e f_{B}}}\right)^{\frac{1}{\alpha_{B}}}} \\
& {\left[\bar{V}_{h r}(623 \mathrm{ft})\right]_{B}=75.4 \times\left(\frac{900}{33}\right)^{\frac{1}{6.5}} \times\left(\frac{623}{1200}\right)^{\frac{1}{4}}=106.4 \mathrm{mph}}
\end{aligned}
$$

\section{Time Scaling}

The following calculations determine the corresponding wind exposure duration for prototype model based on each second wind exposure of the model in the wind tunnel testing.

$$
\begin{aligned}
& \lambda_{t}=\frac{\lambda_{l}}{\lambda_{v}} \\
& \lambda_{l}=\frac{0.4 \mathrm{~m}}{620 \mathrm{ft}}=\frac{0.4 \mathrm{~m}}{189 \mathrm{~m}}=\frac{1}{472.5} \\
& \lambda_{v}=\frac{30 \mathrm{mph}}{106.4 \mathrm{mph}}=1 / 3.54 \\
& \lambda_{t}=\frac{\lambda_{l}}{\lambda_{v}}=\frac{1 / 472.5}{1 / 3.55}=1 / 133
\end{aligned}
$$

Database Time history Time Step $=0.00195 \mathrm{~s}(512 \mathrm{~Hz})$

Scaled Time Step $=0.0195 s \times 133=0.26 s$

Resampling to $4==>$ S caled Time Step $=\frac{0.26}{4}=0.065 \mathrm{~s}$

\subsubsection{Wind Time-History Point Loads}

After calculating the wind pressure time-histories related at each pressure tap location, the corresponding time-history point loads were obtained by using the following equation: 
$F_{i}=P_{i} \cdot A_{i}=C_{p_{i}} \cdot \frac{1}{2} \cdot \rho \cdot V^{2} \cdot A_{i}$

where $A_{i}$ is the surface area corresponding to the $i^{\text {th }}$ pressure tap. For the wind tunnel data provided by either WOW or TPU database, the pressure taps are uniformly distributed over the building's surface. Therefore, $A_{i}$, identical for all points, was easily obtained by dividing the whole area by the number of the pressure taps.

The time-history point loads for all pressure tap locations were obtained as described above. But there was a challenge that pressure taps were located inside the frame panels and the finite elements model was consisting of the only beams and columns. Therefore, it was required to find the equivalent time-history point loads on the beam-column junctions according to available inside-panel wind point loads (as shown in Figure 5-15). To this end, for each single inside-panel point load, the four load components at the corners of the enclosing panel were calculated. Then, through the time-domain summation of the loads in each beam-column junctions, the wind timehistory point loading for each beam-column junction was obtained.

For 47-story, 40-story and 30-story buildings the wind loading distributions were simulated through 256 (64 on each face), 208 (52 on each face) and 160 (40 on each face) time-history point loads around the building respectively, Figure 5-17.
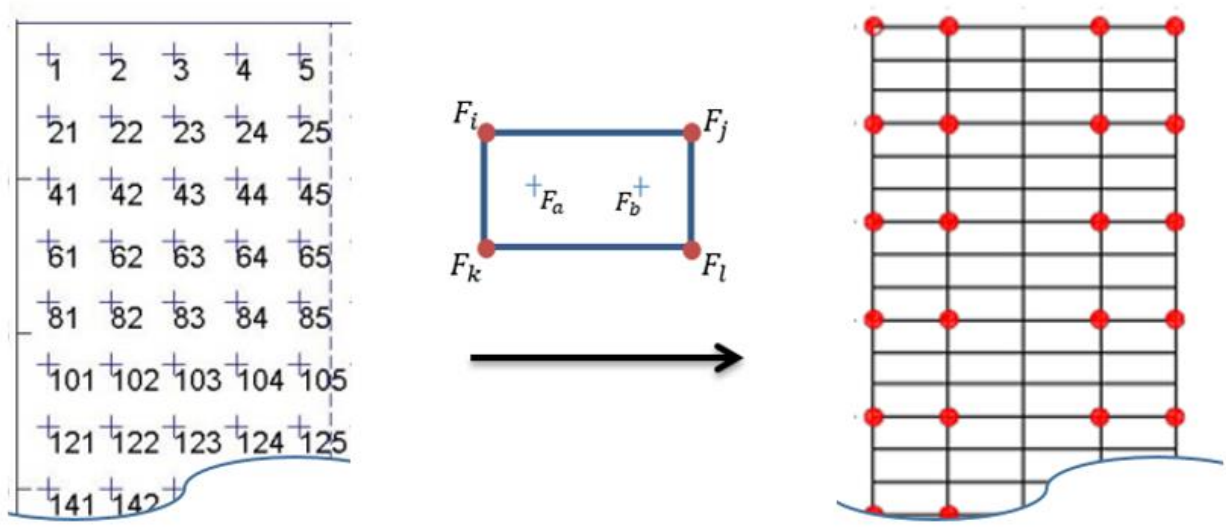

Figure 5-15. Converting measured loads to the location of the applied time-history point loads 


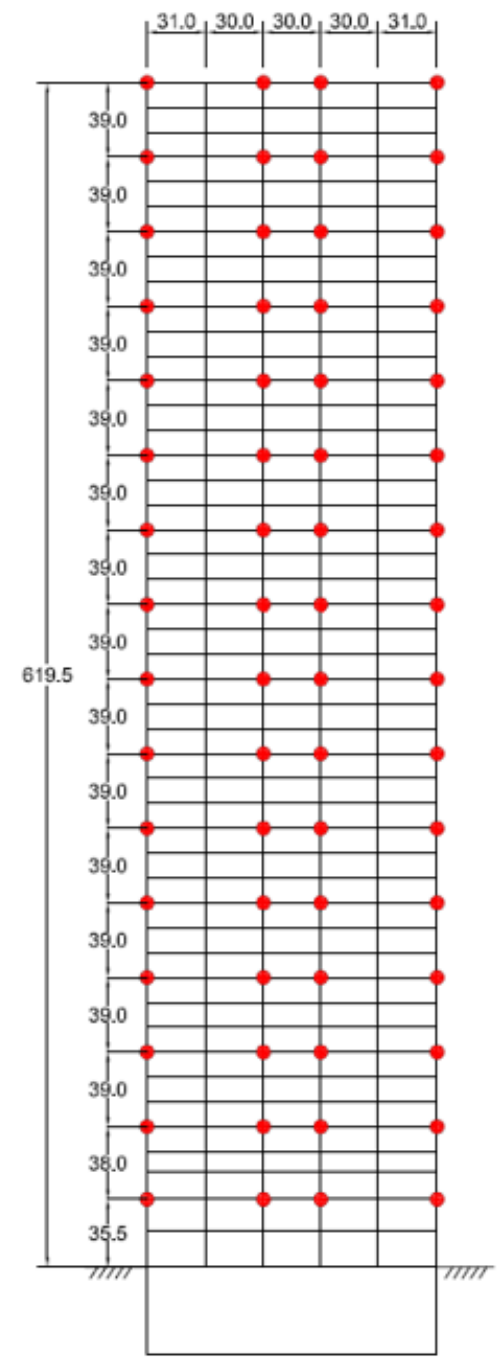

47-Story Building

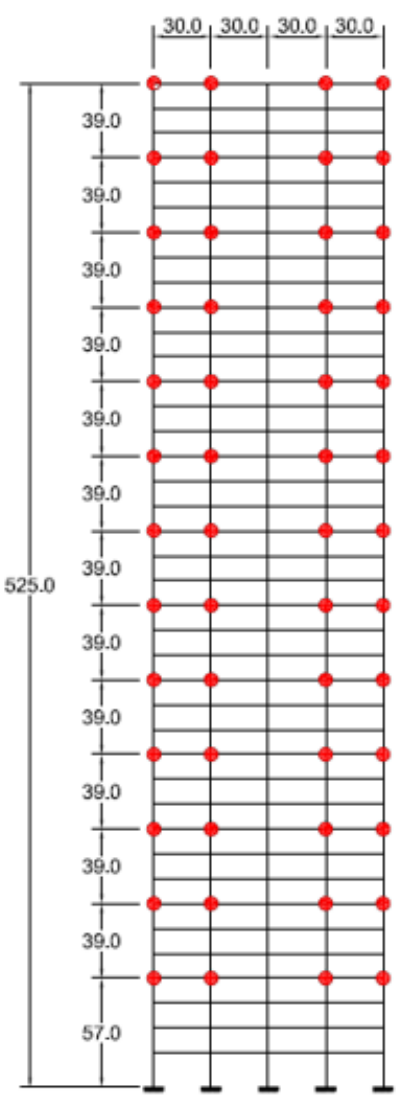

40-Story Building

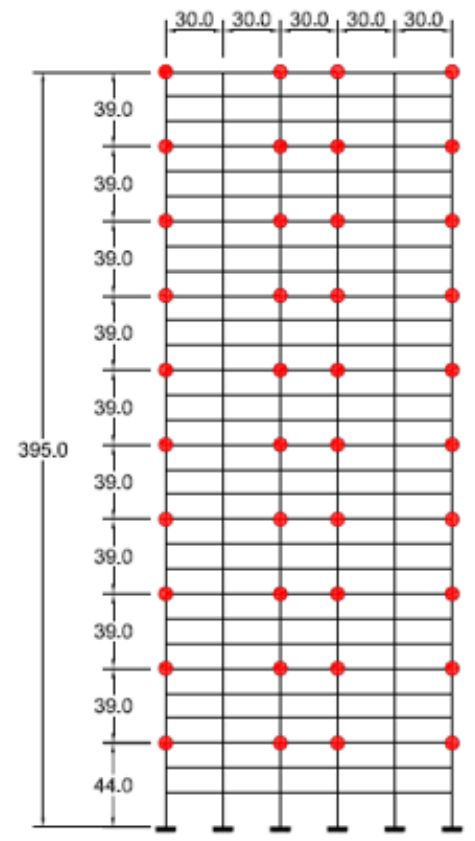

30-Story Building

Figure 5-16. Location of applied time-history loads on each building's face for all three buildings

Figure 5-17 shows the typical time-history point load used in this study. As shown in the figure, a ramp-up also added to the beginning of time-histories, gradually increasing the forces in first several seconds, from zero to the initial amount. It was to avoid the dynamic impact effect influencing the dynamic response of the building. 


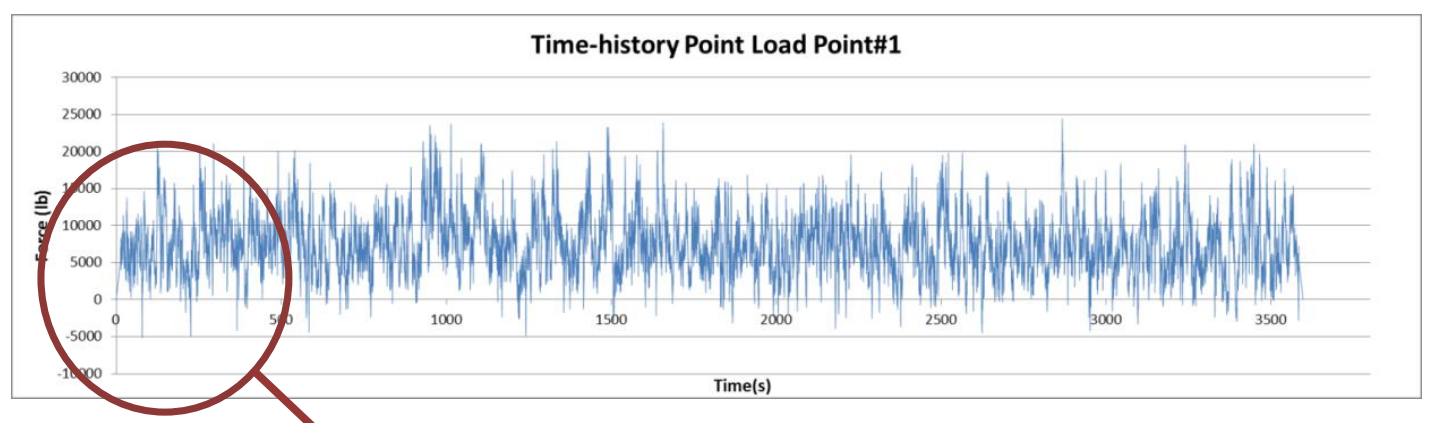

(a)

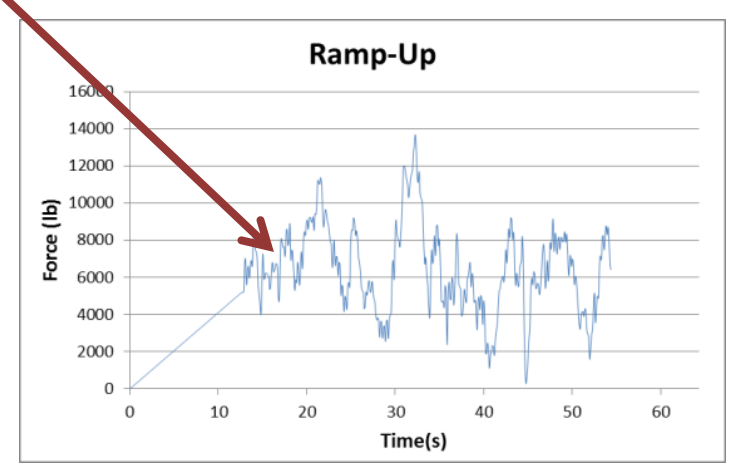

(b)

Figure 5-17. Wind time-history loading; (a) typical point load, (b) the added ramp-up portion 


\section{CHAPTER VI}

\section{ANALYSIS RESULTS}

\subsection{Introduction}

This chapter presents the finite element analysis results of three steel moment frame high-rise buildings investigated in this research. The 3D finite element models of the buildings were developed in accordance with the analytical approach explained in Chapter II. The wind dynamic loading data for the existing 47-story building was acquired by conducting wind pressure testing on a scale model as described in chapter V. For the two other buildings the wind dynamic loading data obtained from TPU Aerodynamic Database, which provides wind pressure database based on wind tunnel experiments on high-rise building models, was used. This chapter consists of sections presenting the results for each building model separately. Each section begins by presenting the modal analysis results, followed by reporting the static nonlinear (pushover) wind analysis results and the estimated static wind over-strength of the building. The wind nonlinear response history analysis results are then explained and the wind directionality characteristics are also discussed. An Incremental dynamic analysis (IDA) approach is implemented to provide a comprehensive estimation of wind nonlinear dynamic response for different levels of wind hazard. It represents the buildings responses as functions of design basic wind speed. The IDA results provide wind structural response data required to conduct the wind performance assessments as presented in the next chapter.

\subsection{Analysis Results of The 47-Story Building}

\subsubsection{Modal Analysis Results}

The basic dynamic response characteristics of the 47-story building were first investigated by conducting the modal analysis. The mode shapes, natural periods and the type (either translational or torsional) of the first five modes of the building are shown in Figure 6-1. As expected the first 
two modes correspond to pure cantilever deformation modes in two principal directions of the building followed by the torsional mode. The relatively low natural frequency of the two first modes indicates the high flexibility of the building which results in large lateral displacement and associated P- $\Delta$ effects which significantly restrict the lateral resistance of the building.

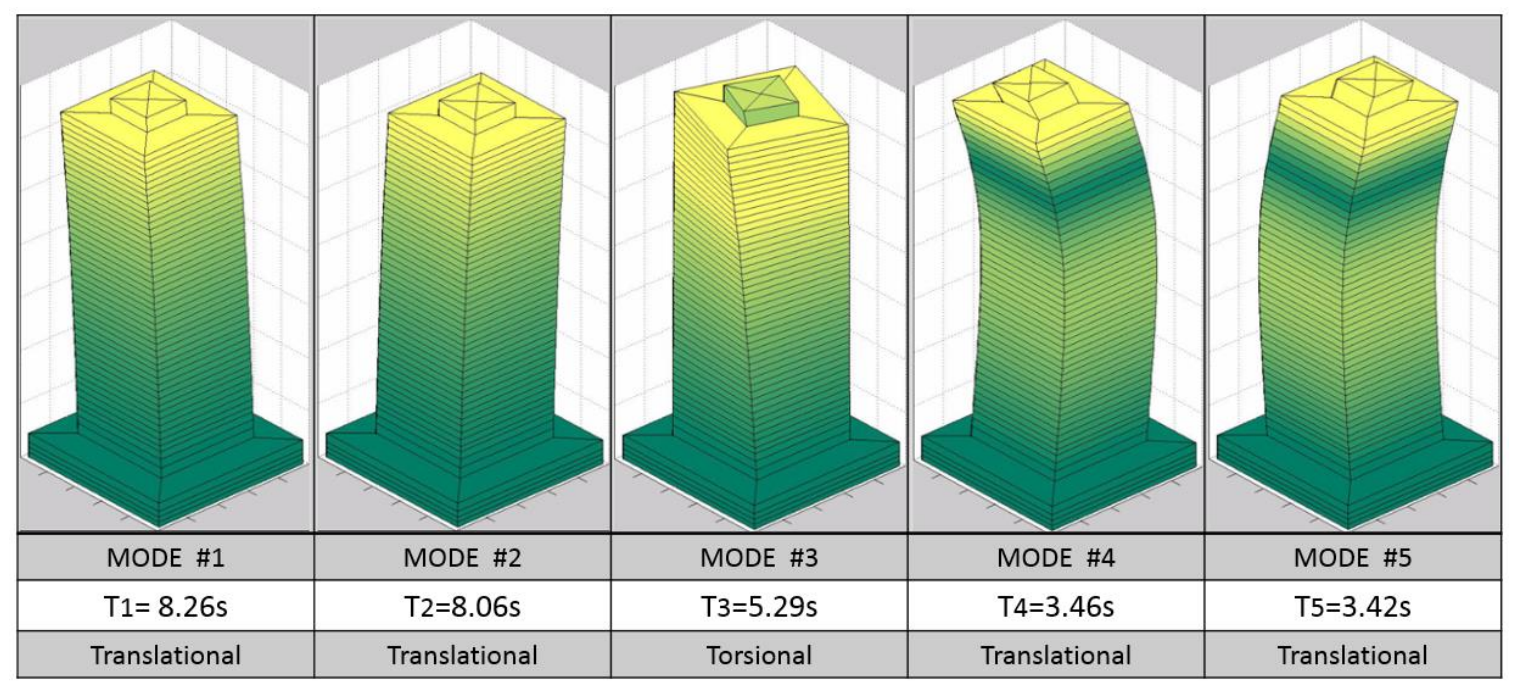

Figure 6-1. Modal Shapes, natural periods and the types of first five modes of 47-story building model

\subsubsection{Nonlinear Static (Pushover) Analysis}

In nonlinear pushover analyses, it was aimed to subject the model of the building to an incremental lateral load with a loading pattern representing the distribution of wind loads on building surfaces during a wind event, to evaluate initiation and distribution of nonlinearity throughout the whole structure. To this end, the ASCE 7-10 wind loading pattern was used to conduct nonlinear pushover analyses. While the realistic wind loading on building surfaces may be very different from the pattern suggested ASCE7 due to several influencing parameters such as wind attack direction and configuration of neighboring buildings, nonlinear pushover analysis may be still helpful to (1) check and debug the nonlinear model, (2) provide an understanding of the initiation and development of inelasticity and the amount of strength and deformation and, (3) since standard one-hour nonlinear wind dynamic analysis is very time-consuming, the wind 
nonlinear pushover can be used to roughly estimate how variations in the component properties and modeling assumptions affect the building response.

In each floor of building models, the ASCE 7-10 wind load was calculated based on pressure distribution given by the code multiplied by the area corresponding to that floor (half the height of below and above stories by the width of the building in that specific surface side). The load was applied to the defined center point, constrained to all other nodes in that floor providing floor's rigid diaphragm. The displacement control point used in nonlinear pushover analyses was the center point of the top level (roof) of the building. The total gravity load was applied prior to pushing building with the incremental lateral load. The P- $\Delta$ geometric nonlinear effect was also considered during nonlinear pushover analysis. Increasing the lateral loads was continued until the structure encountered instability and accordingly the analysis stopped converging.

Nonlinear static pushover wind analyses of the 47-story building were carried out in two principal directions of the building. In these analyses, the model was subjected to gravity loads and scaled incremental static wind loading in accordance with ASCE 7-10 directional procedure (chapter27), case 1, applied to all above-ground stories. The pushover analyses were displacement-controlled and continued until lateral instability and collapse of the building. The pushover base shear- roof drift ratio curves obtained for two principal directions of the building $\mathrm{X}$ and $\mathrm{Z}$ (compared to coordination introduced in Figure 5-5, the Y-direction was replaced with Z-direction for consistency with the finite element model coordination) are shown in Figure 6-2a and Figure 6-2b respectively. Locations of plastic hinges for different types of nonlinearity for both pushover analyses in two major directions $\mathrm{X}$ and $\mathrm{Z}$ are shown in Figure 6-3 and Figure 6-4 respectively.

For X-direction response, extensive nonlinearity in beams between levels $10^{\text {th }}$ to $30^{\text {th }}$ and several middle column hinging all in perimeter moment frames are shown in Figure 6-3. The significant contribution of gravity connections also is evident in the near-collapse stage. The collapse 
mechanism in X-direction pushover is produced by a combination of beams and column hinging in the $6^{\text {th }}$ to $12^{\text {th }}$ stories.

For Z-direction response, the beam nonlinearity is spread through all stories and corner column plastic hinging is significant. Gravity framing contribution was relatively less than for the Xdirection response. Considering an almost symmetric framing, the difference between responses in two direction results from the presence of asymmetric corner columns with a weak axis parallel to Z-direction. In the Z-direction pushover response the collapse mechanism is produced by column hinging within the $2^{\text {nd }}$ story level.

The wind design base shear according to contemporary local building code identical in both building direction was 2830 kips. As shown in Figure 6-2, the nonlinear response of the building resulted in ultimate lateral resistance equal to 7090 and 7065 kips for the $\mathrm{X}$ and $\mathrm{Z}$ directions respectively. Wind over-strength in the building's two major directions, $\Omega_{\text {wind }}$ and $\Omega_{\text {wind }}$, defined as the ultimate lateral resistance capacity (obtained from nonlinear static pushover wind analysis) to the wind design base shear, was equal to 2.50 . Since the building had not been apparently designed for drift limits, this over-strength can be attributed to remarkable nonlinear capacity and contribution of gravity framing, in addition to other factors such as design efficiency and inherent conservatism in the design approach. 


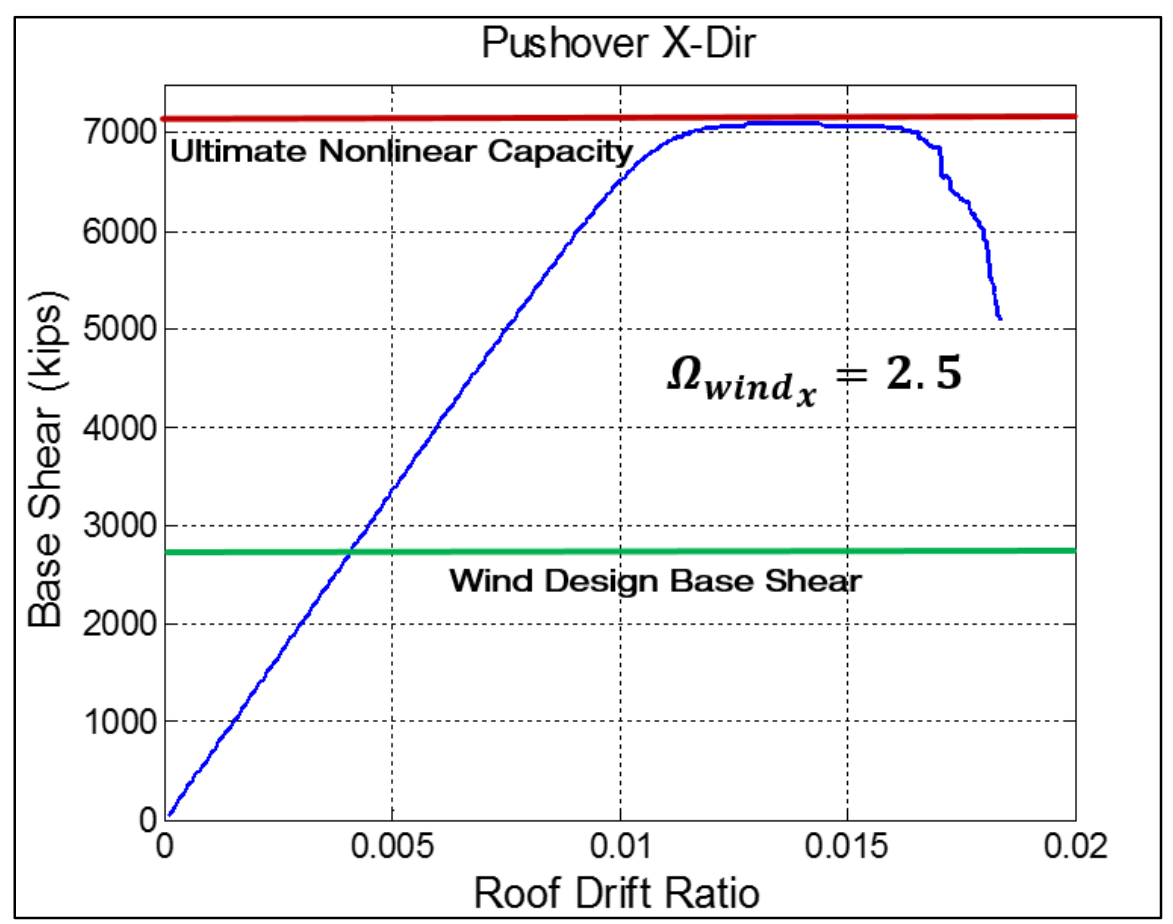

(a)

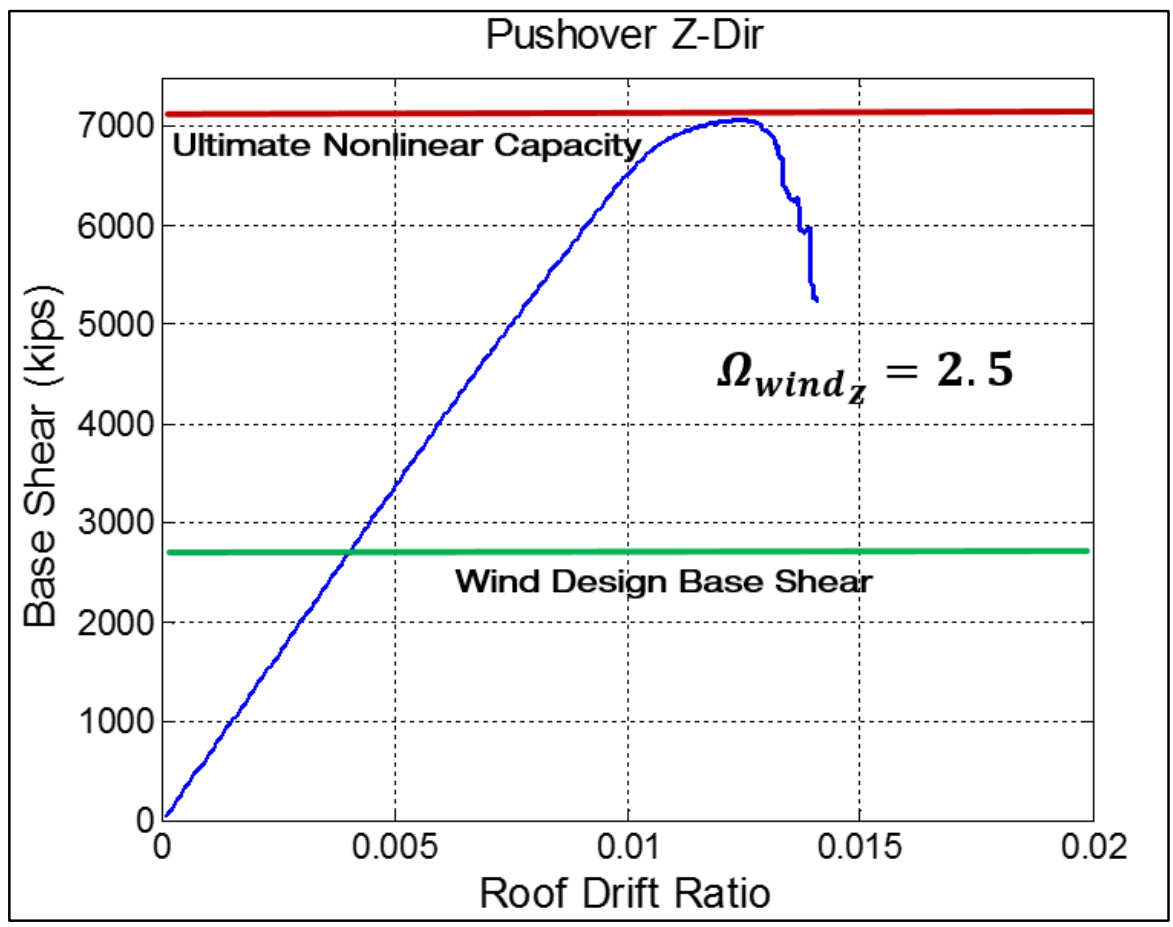

(b)

Figure 6-2 . Wind nonlinear pushover: (a) Pushover curve for X-direction response; (b) Pushover curve in Z-direction response 
- Beam Plastic Hinge - Column Plastic Hinge - Panel Zone Plastic Hinge

- Pre-Northridge Connection Plastic Hinge - Gravity Connection Plastic Hinge

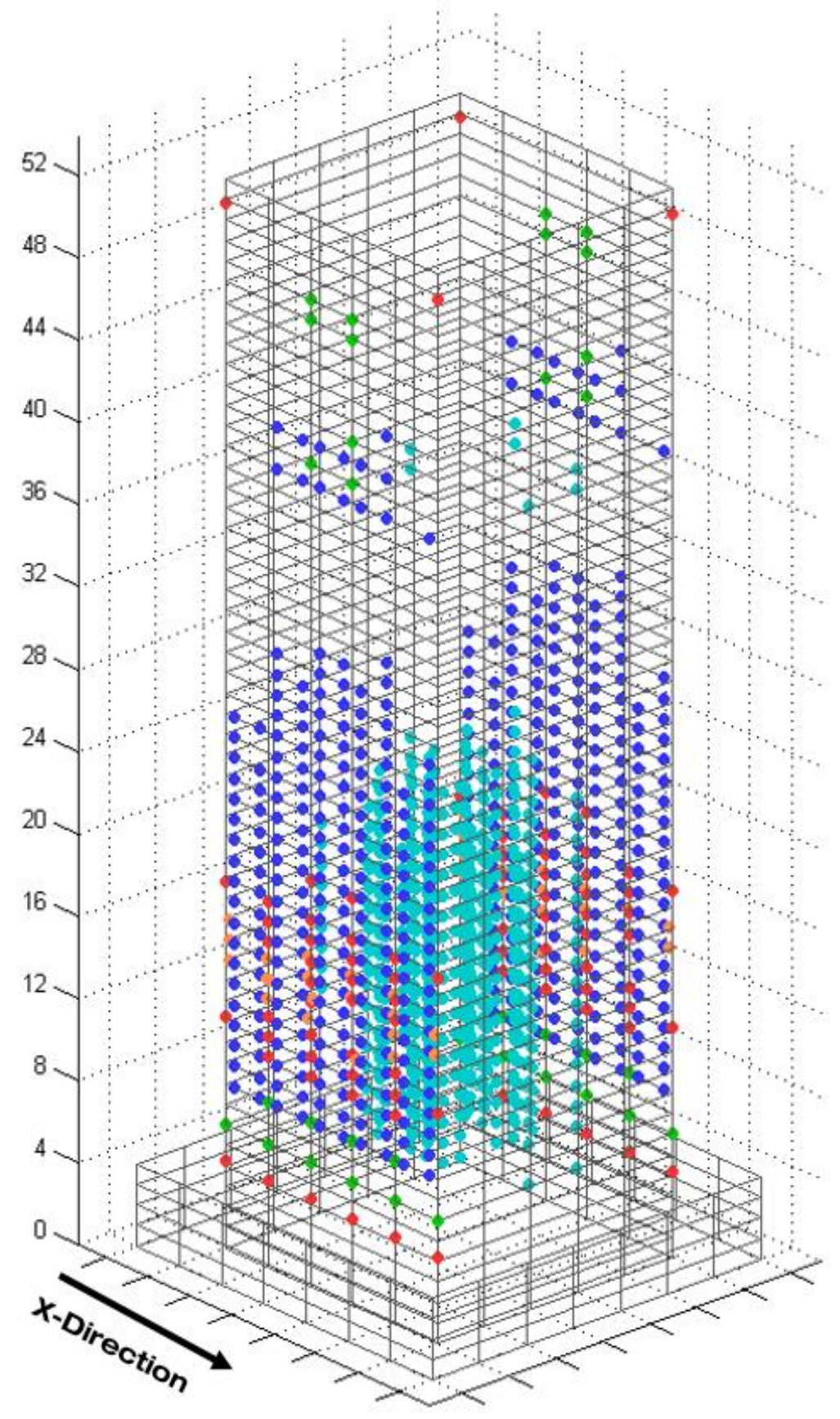

Figure 6-3. Wind pushover response of the 47-story building; nonlinearity distribution in nearcollapse stage in $X$-direction response 
- Beam Plastic Hinge Column Plastic Hinge - Panel Zone Plastic Hinge

- Pre-Northridge Connection Plastic Hinge - Gravity Connection Plastic Hinge

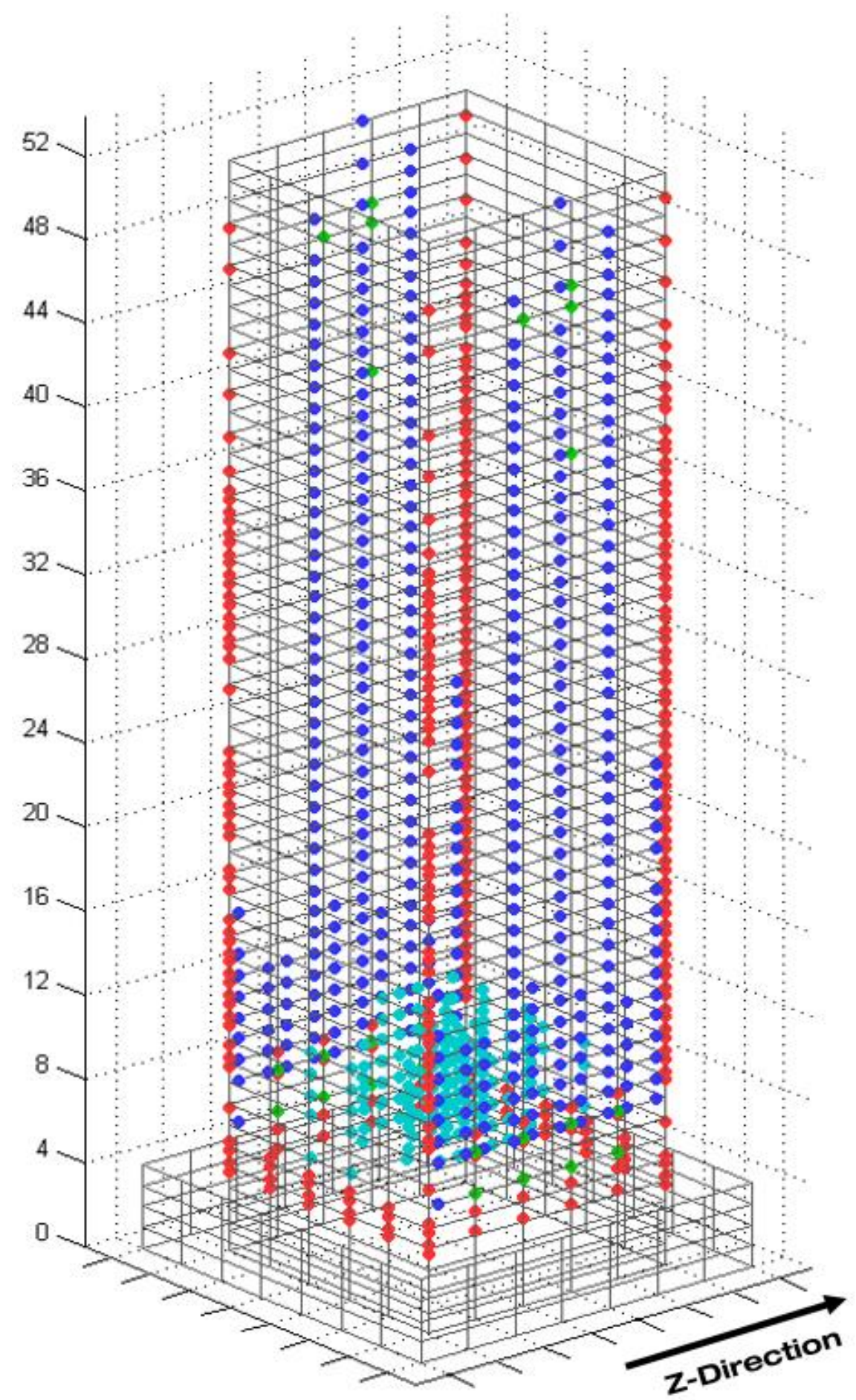

Figure 6-4. Wind pushover response of the 47-story building; nonlinearity distribution in nearcollapse stage in Z-direction response 


\subsubsection{Nonlinear Response History Analysis Results}

Nonlinear Response History analysis is the most reliable analysis currently available to evaluate the response of structures to dynamic excitations such as earthquake and wind events. This analysis improves the response prediction by incorporating explicit nonlinear behavior of structural components as well as reducing the uncertainties associated with structure and load dynamic characteristics. Therefore, it can provide the most realistic response and consequently most reliable performance assessment of a structure subjected to the dynamic excitations. Since the nonlinear dynamic analysis incorporates inelastic member response to cyclic wind loading, it needs to explicitly simulate hysteresis energy dissipation of each nonlinear component. The nonlinear dynamic analysis ideally intends to simulate the full-range of structural response from the linear response to the onset of nonlinearity and corresponding induced damage and finally the collapse of the building. However, more related experimental data and enhanced analytical capabilities are still required to provide more precise simulations of dynamic responses of nonlinear structural systems. Generally, there are two basic approaches for solving dynamic equilibrium equations of a nonlinear system: Direct integration and Modal Response History.

\section{Nonlinear Modal Response History Method}

The Nonlinear Modal Response History Method also known as Fast Nonlinear Analysis (FNA) is a modal analysis method to solve nonlinear dynamic equilibrium equations. In this approach, for each time step the modal equations are uncoupled and solved and the forces in nonlinear elements are calculated by integrations at the end of the time step. This is an efficient method for nonlinear response history analyses for mostly elastic structures with a limited number of nonlinear elements/links (Wilson, 2002). Using the FNA method required the following considerations to assure that the modal forces are able to represent the nonlinear forces:

1) All nonlinear degree of freedoms should be associated with a mass or mass moment of inertia 
2) For modal analysis, the Ritz vector method should be used,

3) The analysis should include a sufficient number of modes to capture at least $90 \%$ mass participation, while it should be assured that the static modal load participation ratio of each nonlinear degree of freedom is $100 \%$.

4) To ensure the convergence to a mathematically accurate solution, sufficiently small time steps should be chosen.

\section{Direct Integration Method}

The Direct Integration Method is based on solving the nonlinear dynamic equilibrium equation by using step-by-step numerical integration procedure. This approach involves the attempt to satisfy the dynamic equilibrium at discrete time intervals. In this approach, the velocity and displacement calculated at the end of each time step are used as the initial condition of the next time step. It allows incorporating the nonlinear nature of the system by updating the component properties corresponding to the deformed shape at the end of each time step. Therefore, this step-by-step method provides a very efficient and compatible solution for nonlinear history analyses. This method is very sensitive to the time step size and a sufficiently small time step, depending on the size and the characteristics of the model, needs to be chosen to ensure the convergence of the analysis.

In this study, the Direct Integration Method of OpenSEES was used to solve nonlinear dynamic equations. An algorithm coded in OpenSEES controls the solution of nonlinear history response analysis. This algorithm adopts the Newmark direct integration method with coefficients $\gamma=0.5$ and $\beta=0.25$ (Average Acceleration Method) as the main method. If the Newmark method fails the algorithm directs the solution to try Newton with Initial Tangent method, Broyden method and finally Newton with Line Search Method in sequence. 
For the 47-Story building, wind nonlinear history response analyses were performed by subjecting the building model to simulated dynamic wind loading in terms of 256 time-history point loads around the building surface. The duration of the analyses was around 4000 to 6000 seconds including one hour of wind loading and a ramp-up portion to avoid dynamic impact effect. A critical damping ratio equal to $2 \%$ using Rayleigh damping method was applied in nonlinear history response analyses. To avoid the spurious damping forces, the damping matrix was assembled based on the tangent stiffness and nonlinear elements were excluded while providing damping to the elements.

Wind directionality characteristics of the wind dynamic response were investigated by conducting a set of response history analyses for wind incidents with a constant basic wind speed of $82 \mathrm{mph}$ and directions of attack varying from $0^{\circ}$ to $360^{\circ}$ at an increment of $15^{\circ}$. Peak story drift ratio and peak base shear responses in two major building directions are shown as functions of wind attack angles in Figure 6-5c and 6-5d. It is shown that the responses roughly follow the loading characteristics as shown in terms of resultant base shear and overturning coefficients directly obtained from wind testing data, shown in Figure 6-5a and 6-5b. As shown in the figure the largest loading and responses are obtained in $120^{\circ}$ to $180^{\circ}$ wind direction range which is considered as the critical wind directions of the 47-story building. 


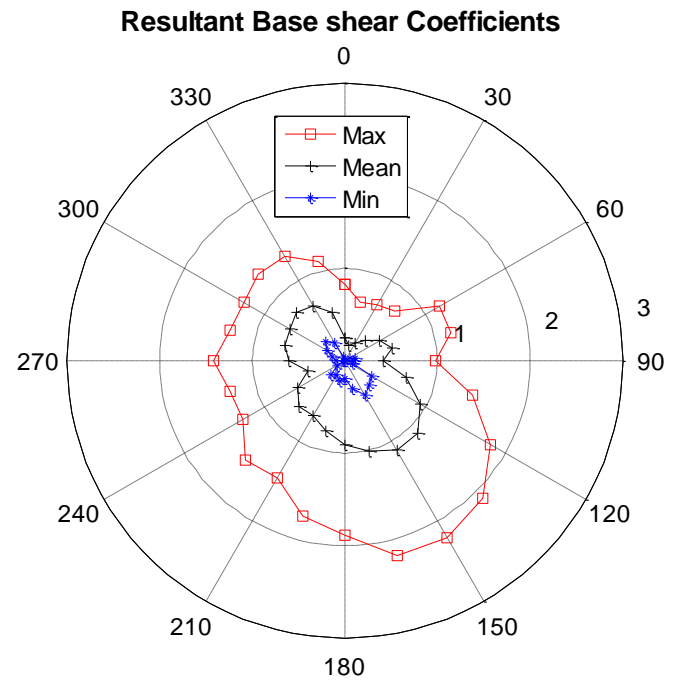

(a)

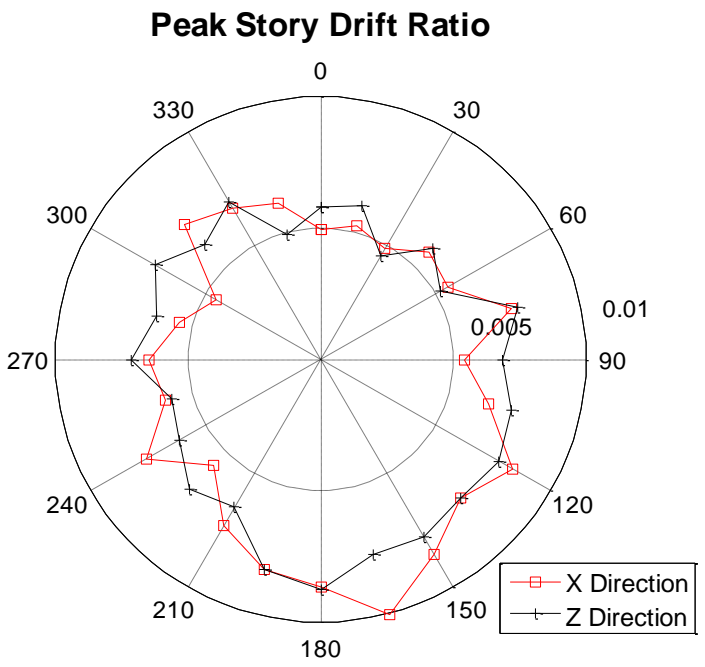

(c)

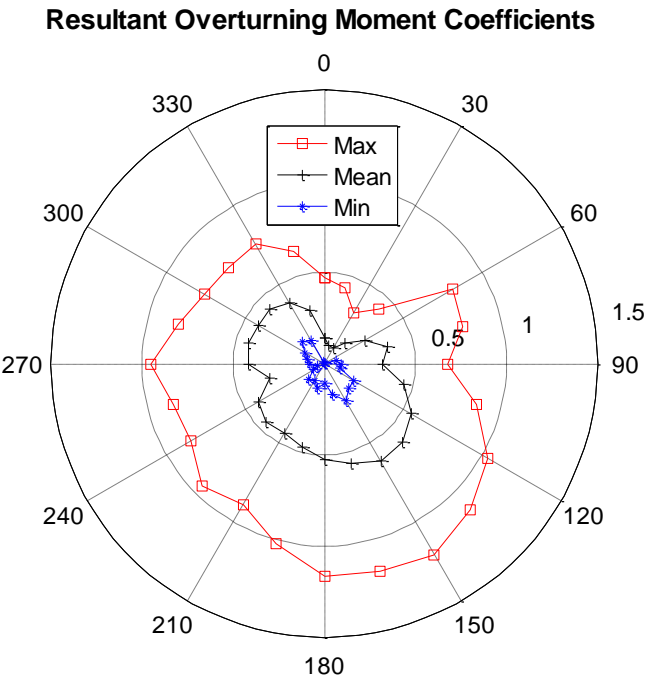

(b)

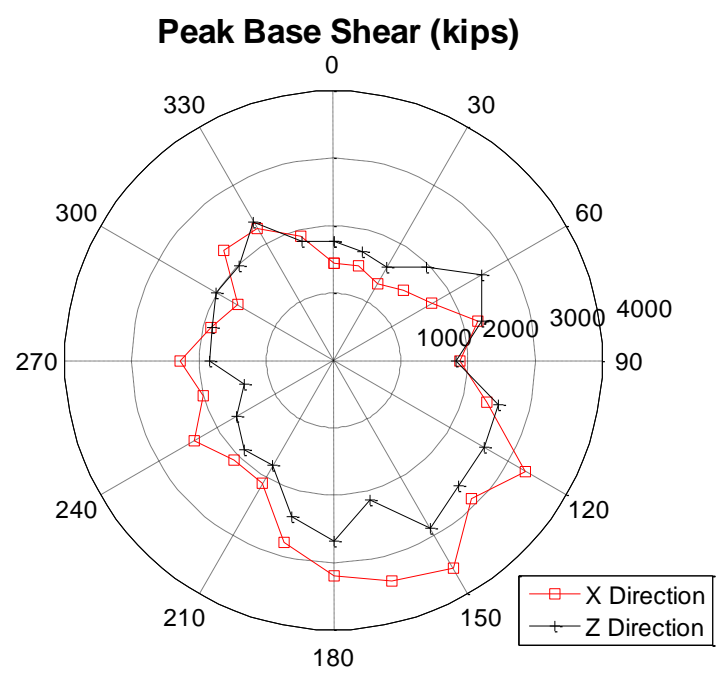

(d)

Figure 6-5. Wind directional dynamic response of the 47-story building: (a) peak story drift ratio; (b) peak base shear

To study both the interference effect of the neighboring buildings and the effect of building shape characteristics on the dynamic response of the building, a set of wind dynamic analyses conducted using wind pressure data obtained from three WOW tests at a constant wind speed of $82 \mathrm{mph}$. In all three analyses, the wind loading at $180^{\circ}$ wind direction was used. The selected wind direction corresponds a relatively open side of the building. Figure 6-6 shows the wind dynamic responses including story drift ratio and peak floor acceleration in both principal 
directions of the building for three different WOW test wind loading data. Besides the illustration of stiffness distribution along the building, the figure shows the effect of the real environment of the building in an almost open direction. It is shown that the along-wind responses are almost consistent, although the cross-wind responses are significantly affected by interference and shape characteristics effects. In this wind direction the building shape characteristics (Test \#2 compared to Test \#1) had a more significant effect than surrounding buildings interference (Test \#3 compared to Test \#2). 


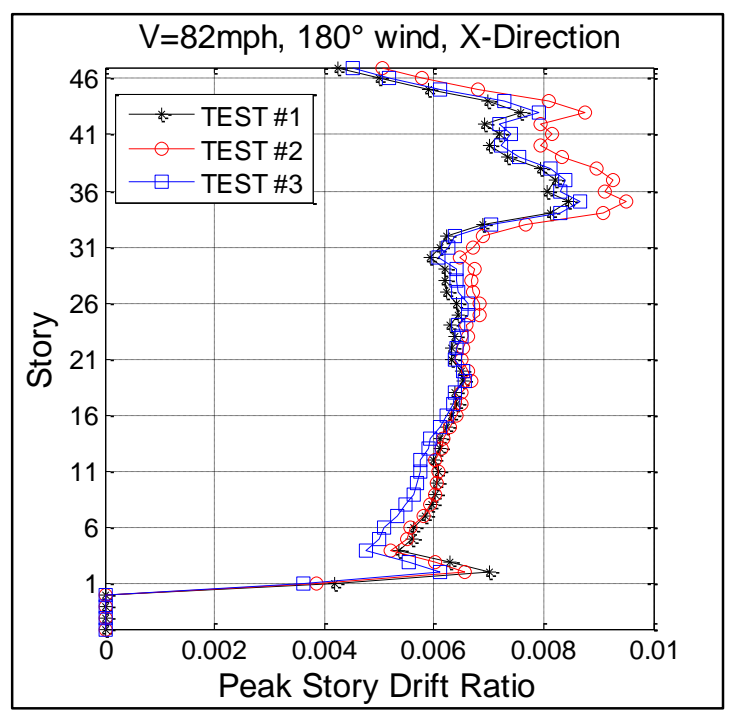

(a)

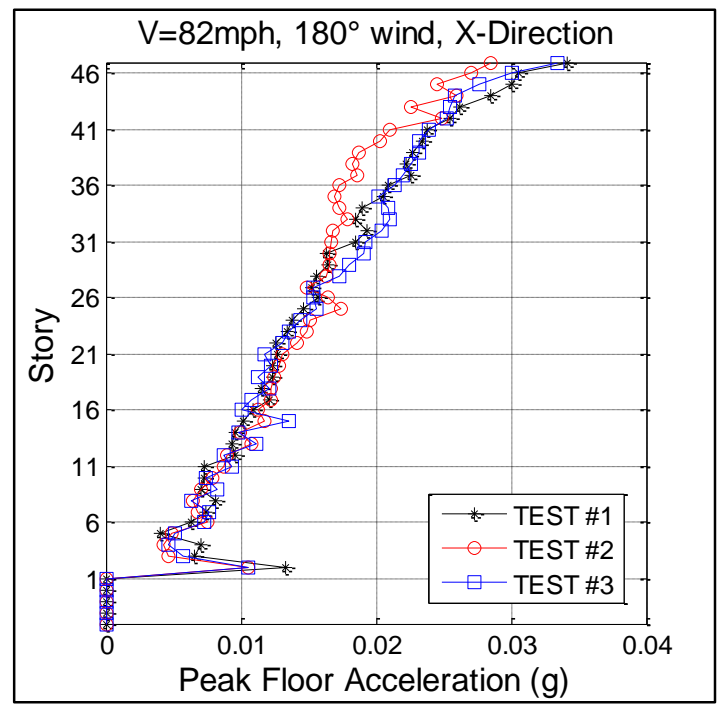

(c)

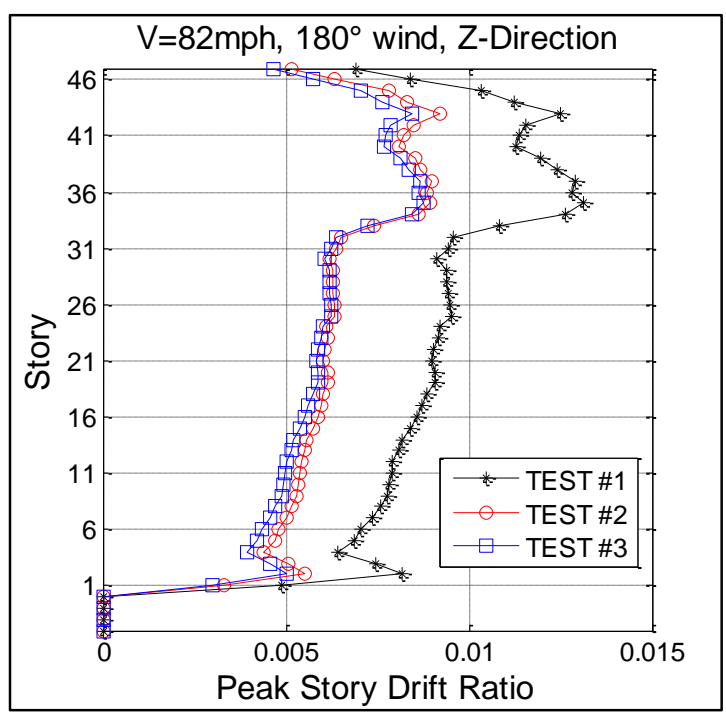

(b)

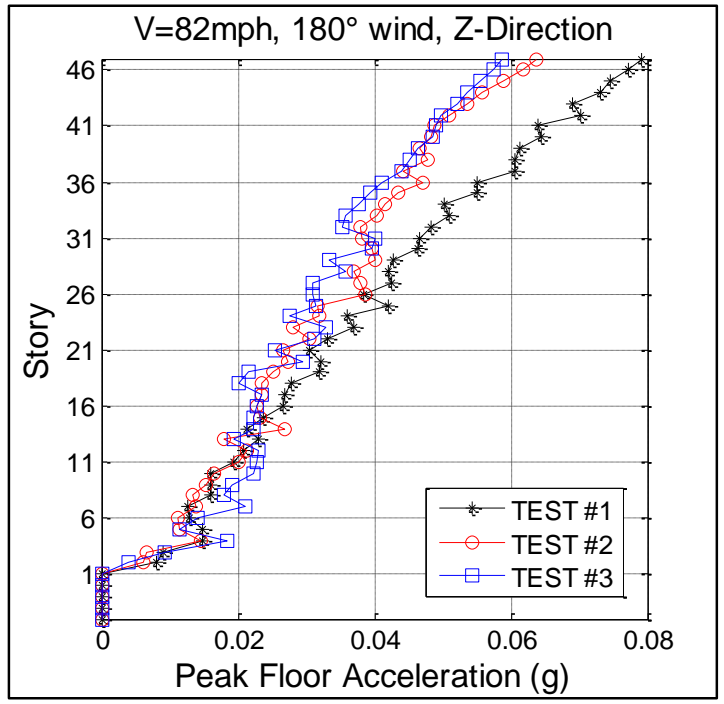

(d)

Figure 6-6. Wind dynamic responses for $V=82 \mathrm{mph}$ wind speed and $180^{\circ}$ wind direction for the three conducted wind pressure tests: (a) peak story drift ratio in X-direction: (b) peak story drift ratio

Z-direction; (c) peak floor acceleration in X-direction: (d) peak floor acceleration in Z-direction

\subsubsection{Incremental Dynamic Analysis (IDA) Results}

Incremental dynamic analysis (IDA) is a modern method of structural analysis which is used to predict the response of the structures under different levels of the dynamic loads. IDA provides a way to evaluate the behavior of the structure at multiple limit states to make sure that appropriate 
levels of performance are provided corresponding to a wide range of potential hazard levels, instead of the design for just the maximum considered dynamic load. IDA is generally performed by incrementally scaling the dynamic loads to higher levels and determining the responses at each intensity level. IDA curves plot the Engineering Demand Parameters (EDPs) with respect to the intensity measures (IMs). In this study, the design basic wind speed (as defined in ASCE 7-10) is used as the scale of the wind hazard and the engineering demand parameters are specific responses of a structure such as: inter-story drifts and peak floor accelerations corresponding to the different intensity measures.

The Incremental dynamic analysis approach was applied in this study to provide a full-range evaluation of the dynamic response of the high-rise building models subjected to wind excitations. The IDAs were carried out at 16 wind loading levels corresponding to basic wind speeds $50,60,65,76,82,86,90,96,100,104,110,114,120,125,130$ and $136 \mathrm{mph}$. Among them, 76, 104, 114 and136 mph corresponded to 10-year, 50-year, 100-year and 700-year Mean Recurrence Intervals (MRI) retrieved from ATC Council web page (http://windspeed.atcouncil.org). According to obtained critical wind directions and considering the fact that the dominant wind direction in Houston is southeast $\left(145^{\circ}-235^{\circ}\right.$ in given coordination), the Incremental dynamic analyses were repeated for six wind directions including $120^{\circ}, 135^{\circ}, 150^{\circ}, 165^{\circ}, 180^{\circ}$ and $195^{\circ}$.

Figure 6-7 shows the IDA curves for peak story drift ratio and MRS floor acceleration for the mentioned wind directions. As shown in the figure collapse due to dynamic wind loading occurs averagely at about $130 \mathrm{mph}$ in accordance with large story sway in the X-direction (roughly considered as the along-wind direction), although the building experiences relatively large drift ratios in the cross-wind direction ( $\mathrm{Z}$ direction). In the aspect of floor acceleration responses, remarkably higher responses are obtained in the cross-wind direction ( $\mathrm{Z}$ direction). 


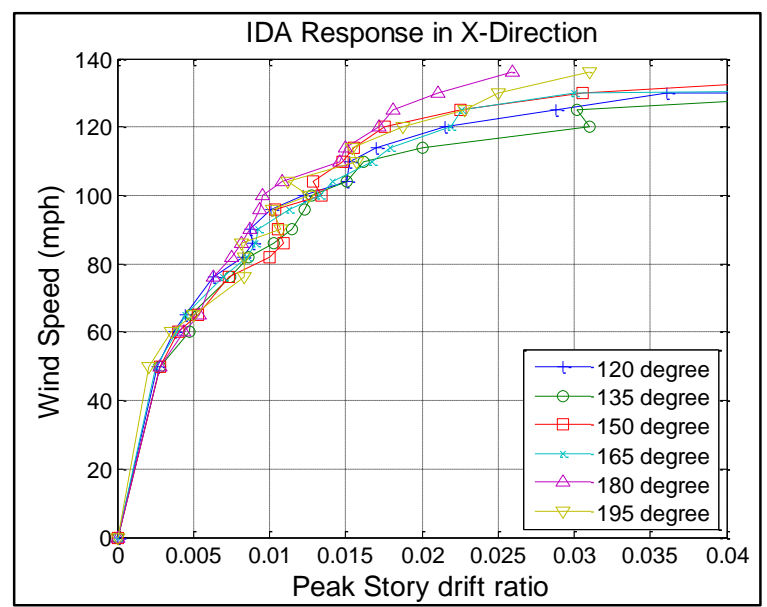

(a)

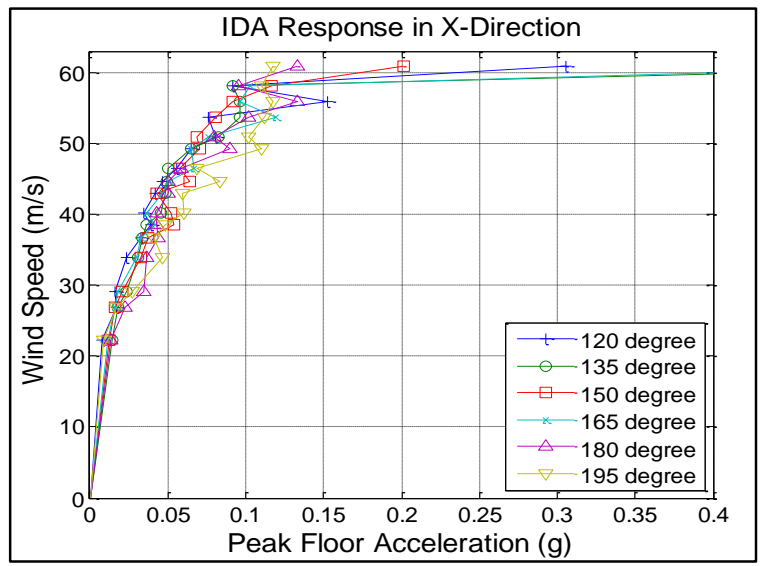

(c)

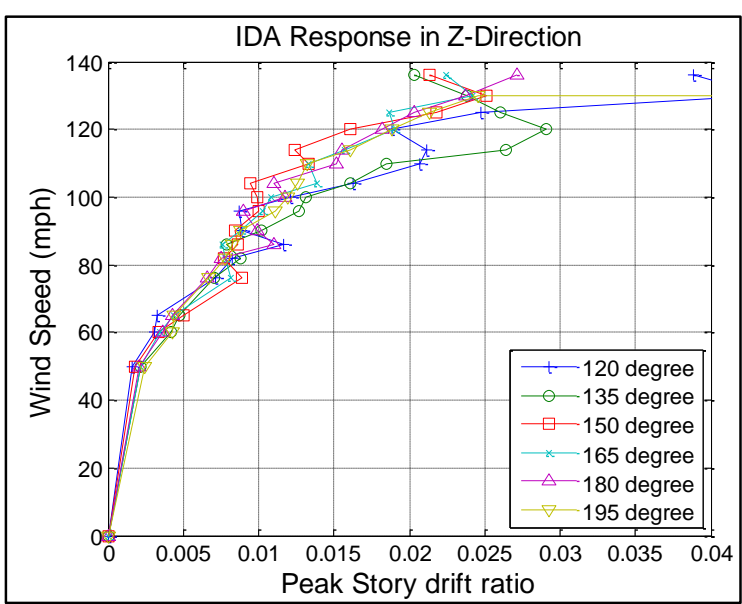

(b)

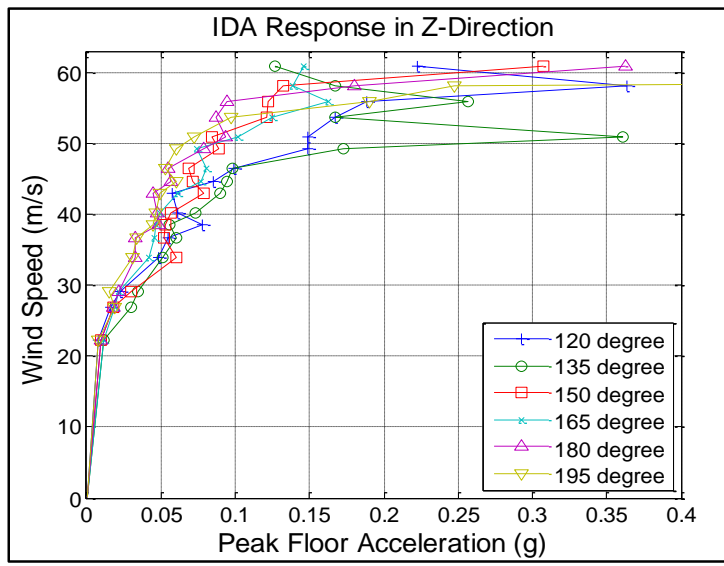

(d)

Figure 6-7. IDA responses of the 47-story building: (a) peak story drift ratio in X-direction; (b) peak story drift ratio in Z-direction; (c) peak floor acceleration in X-direction; (d) peak floor acceleration in Z-direction

\subsection{Analysis Results of The 30-Story Building}

\subsubsection{Modal Analysis Results}

The Modal analysis was first conducted to evaluate the basic dynamic response characteristics of the 30-story building. The mode shapes, natural periods and the type (either translational or torsional) of the first five modes of the building are shown in Figure 6-8. The typical sequence of pure cantilever modes with around 5 seconds period in two building's principal directions, followed by the torsional mode with around 4 seconds periods and finally second modes in the major directions with around 2 seconds periods is observed in the modal response of the building. 


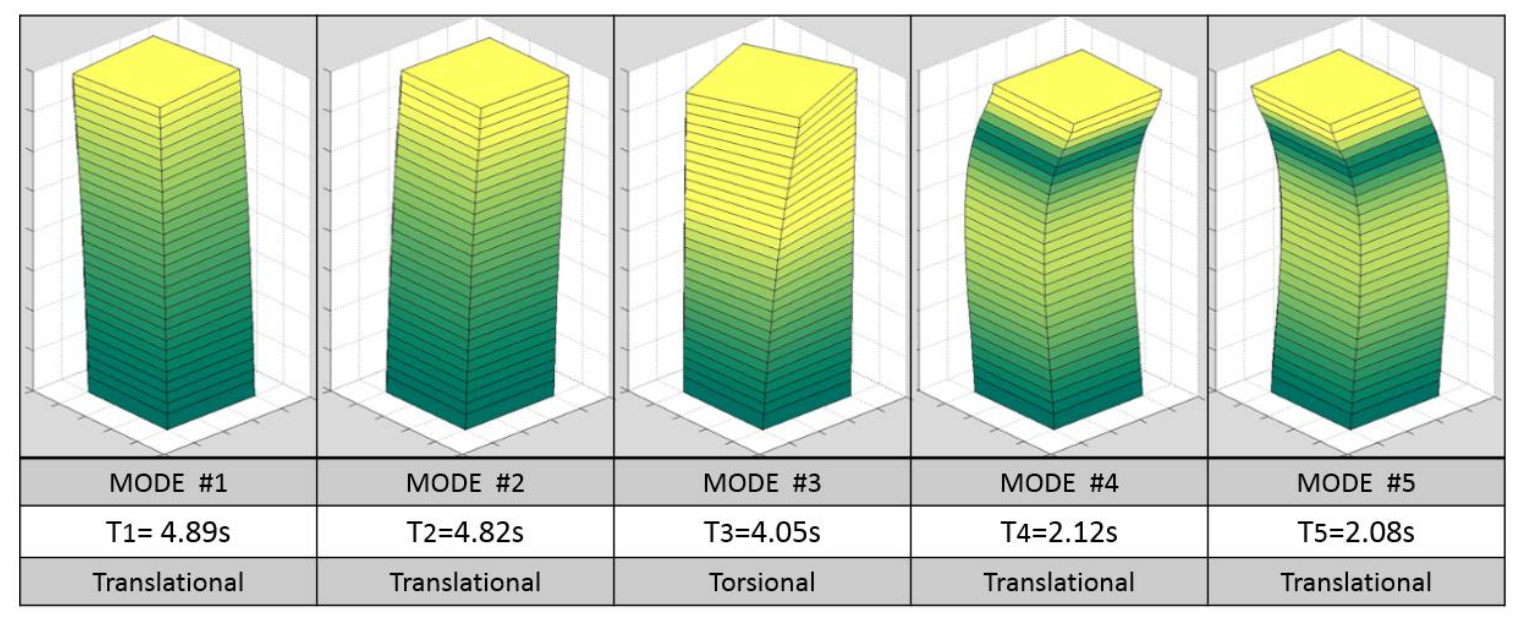

Figure 6-8. Modal Shapes, natural periods and the type of the first five modes of the 30-story building

\subsubsection{Nonlinear Static (Pushover) Analysis Results}

Nonlinear static pushover wind analyses of the 30-story building were carried out in the two principal directions of the building by subjecting the building model to gravity loads and a scaled incremental static wind loading in accordance with ASCE 7-10, case 1 directional wind loading. The pushover analyses were continued until lateral instability and finally collapse of the building. The base shear- roof drift ratio pushover curves obtained for the two principal directions of the building ( $\mathrm{X}$ and $\mathrm{Z}$ direction) are shown in Figure 6-9a and 6-9b respectively. The locations of the plastic hinges corresponding to the different types of nonlinearity in both pushover analyses (in the two major directions of $\mathrm{X}$ and $\mathrm{Z}$ ) are shown in Figure 6-10 and Figure 6-11 respectively. In the both direction responses, extensive panel zone yielding is shown which is due to the bare column webs with no web doubler plate in panel zone area. Significant beam and column plastic hinging in upper levels ranged from $18^{\text {th }}$ to $30^{\text {th }}$ story are also shown for both pushover responses. The contribution of gravity framing is also found to be significant as evidenced by extensive gravity connection plastic hinging. With almost the same response in two directions more beam and column hinging in Z-direction response are shown which can be attributed to longer analysis convergence in that direction. It can be also shown in pushover curves shown in Figure 6-9. The 
wind design base shear according to ASCE 7-10, identical in both building direction, was equal to 2820 kips. As shown in Figure 6-9, the nonlinear response of the building resulted in ultimate lateral resistance equal to 10700 kips, identical in both $\mathrm{X}$ and $\mathrm{Z}$ direction responses. Wind overstrength in two building major directions $\Omega_{w i n d_{x}}$ and $\Omega_{w i n d_{z}}$, was identically equal to 3.7 . The significant over-strength may be attributed to the fact that the seismic loading had governed the design of the building. 


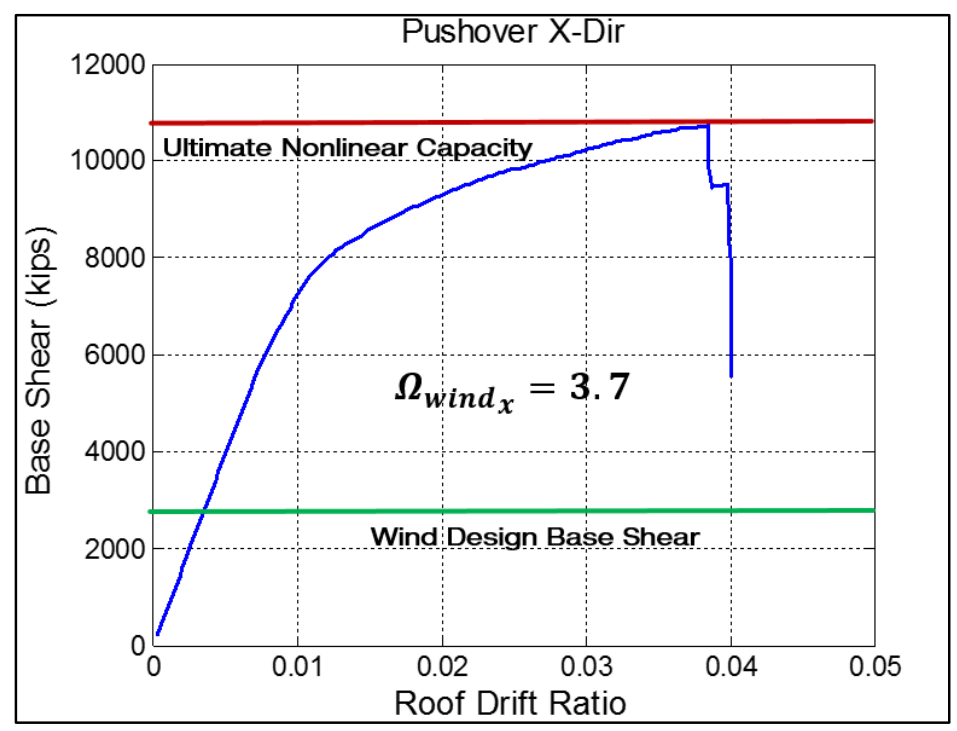

(a)

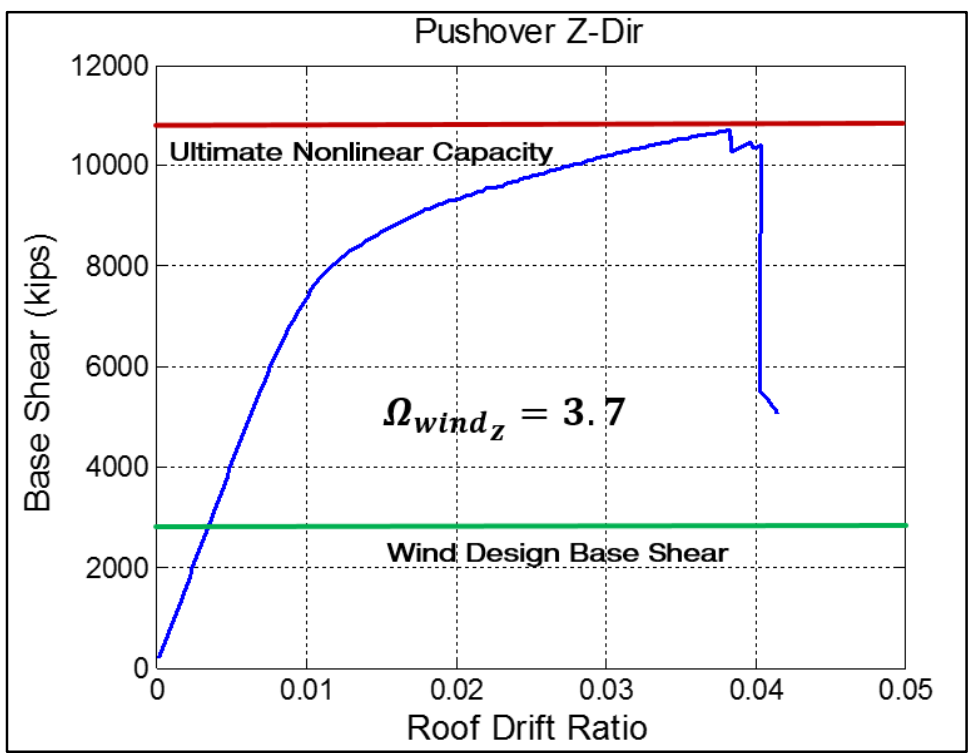

(b)

Figure 6-9. Wind pushover: (a) pushover curve in X-direction response; (b) Pushover curve in Zdirection response 
- Panel Zone Plastic Hinge - Gravity Connection Plastic Hinge

- Beam Plastic Hinge - Column Plastic Hinge

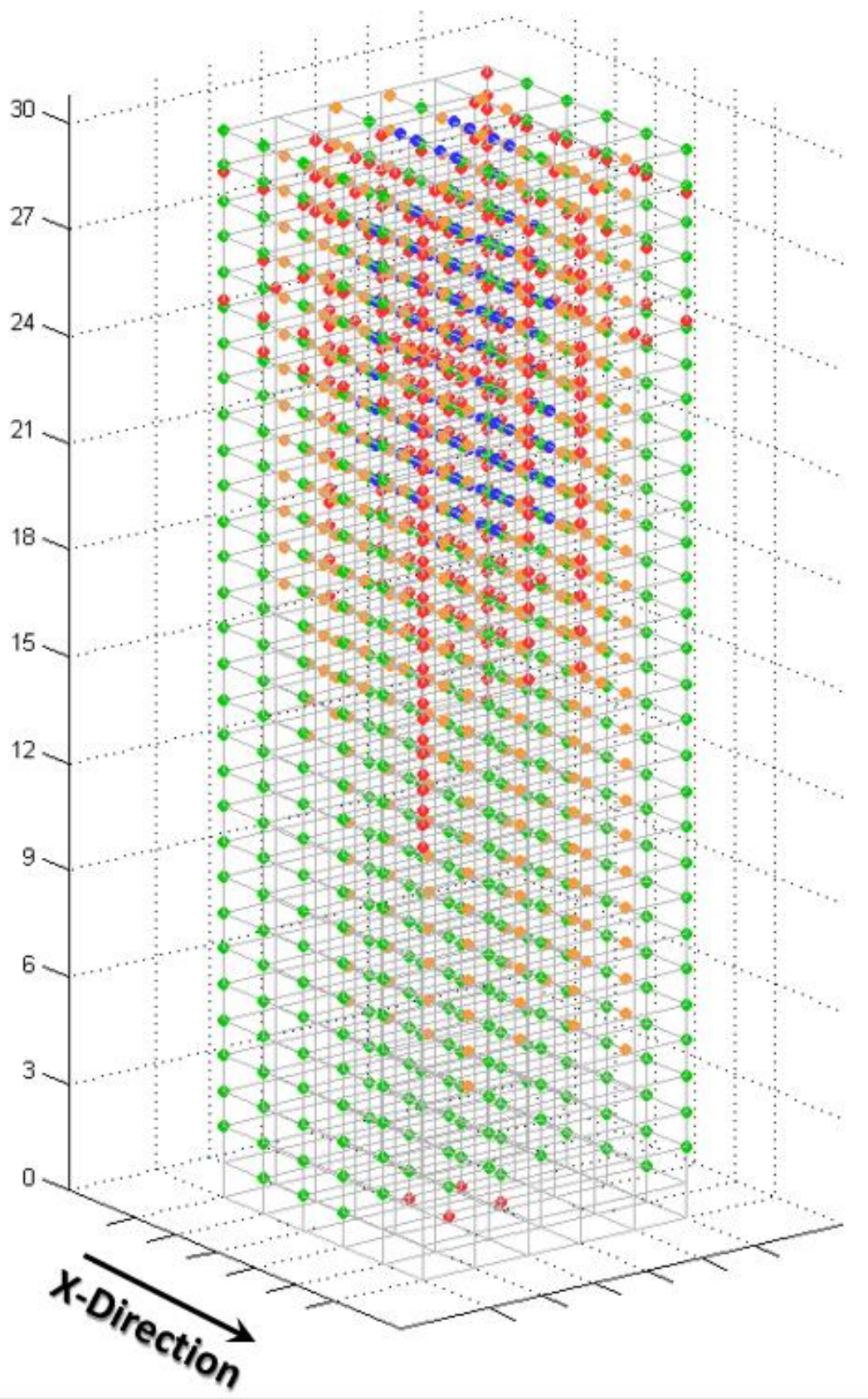

Figure 6-10. Wind pushover response of the 30-story building; nonlinearity distribution in nearcollapse stage in $\mathrm{X}$ direction response 
- Panel Zone Plastic Hinge - Gravity Connection Plastic Hinge

- Beam Plastic Hinge - Column Plastic Hinge

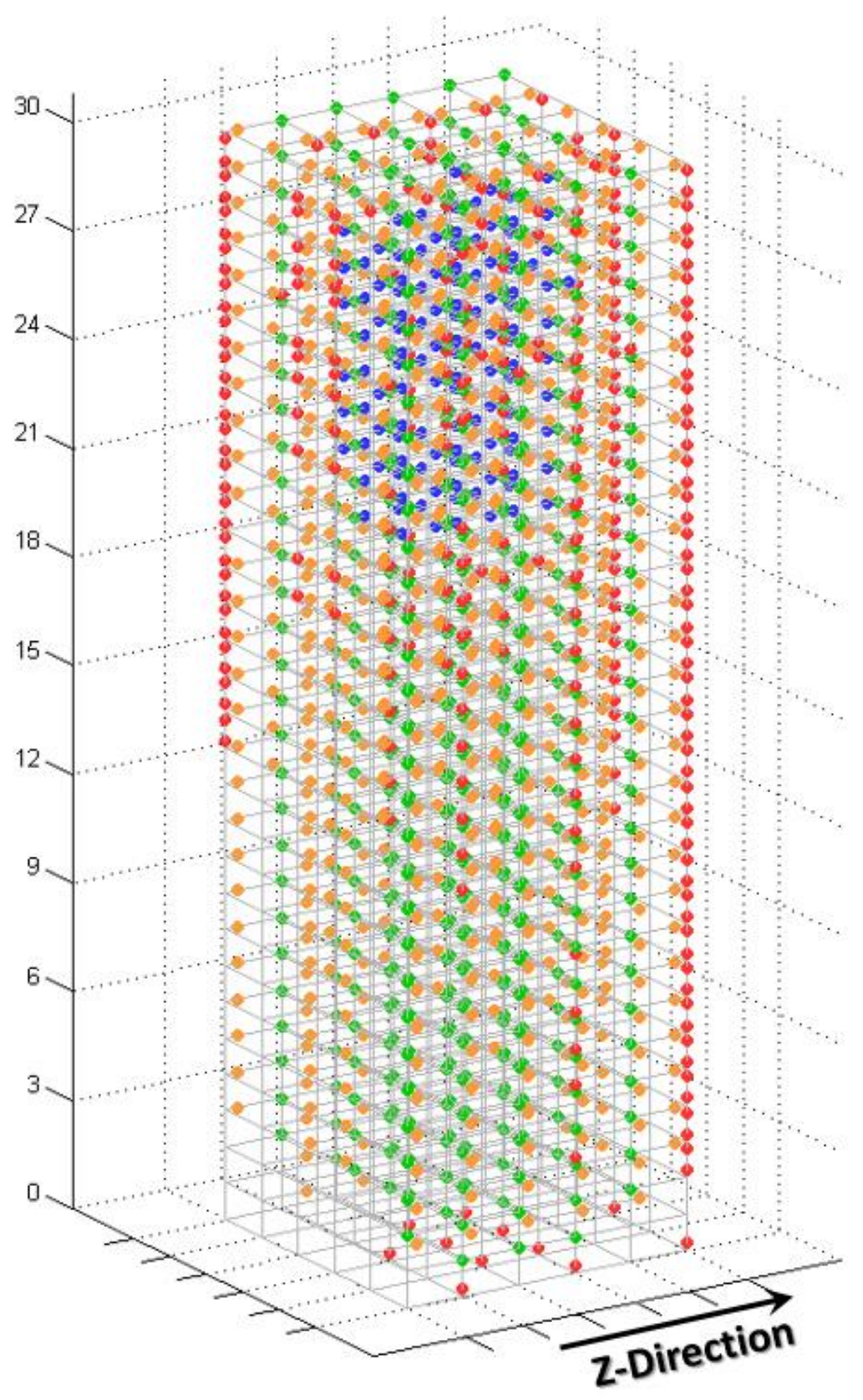

Figure 6-11. Wind pushover response of the 30-story building; nonlinearity distribution in nearcollapse stage in Z-direction response 


\subsubsection{Nonlinear Response History Analysis Results}

Wind nonlinear history response analyses were performed by subjecting the 30 -story building model to the simulated dynamic wind loading in terms of 160 time-history point loads around the building surface. A critical damping ratio of $2 \%$ was applied using Rayleigh damping model. The nonlinear elements were excluded during damping assignment, in order to avoid associated unrealistic damping forces.

The wind directionally effects on the dynamic response of the building were studied by considering the fact that the TPU wind tunnel data is obtained for an isolated wind tunnel model and it does not reflect the effects associated with interference of neighboring buildings. Figure 6-12 and Figure 6-13 represent the dynamic responses including the peak story drift, peak base shear and peak floor acceleration for the wind loading related to the basic wind speed of $76 \mathrm{mph}$ represented as a function of the wind direction. For a symmetrical building like the 30-story building using the isolated wind tunnel data, the responses from $0^{\circ}$ to $45^{\circ}$ represent the wind responses for all the wind directions around the buildings. As shown the figures, for 0-degree wind response the cross-wind peak response is almost as significant as the along-wind response. It is shown that by changing wind direction from $0^{\circ}$ to $45^{\circ}$ a decreasing trend in the both responses is evident. 


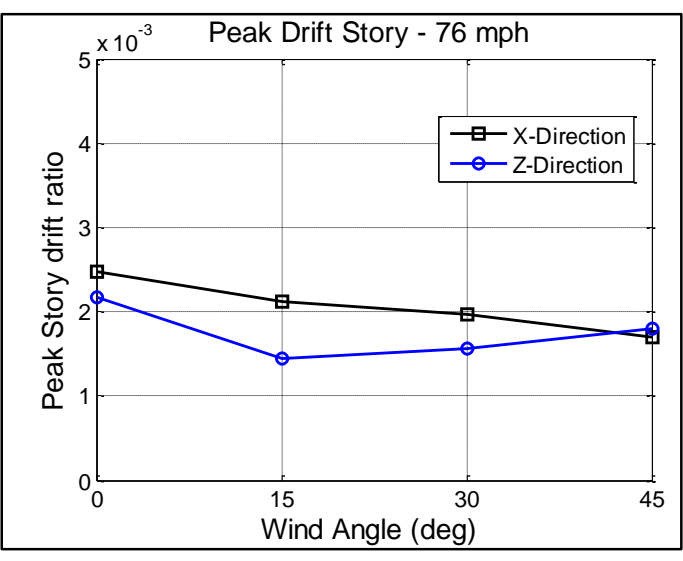

(a)

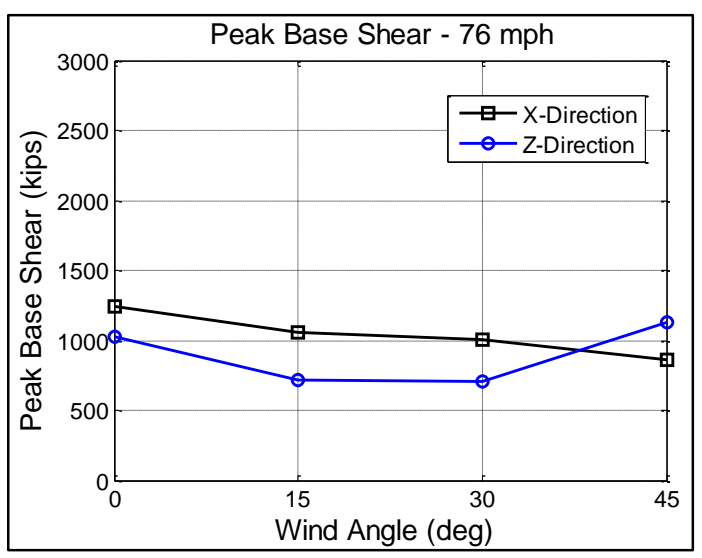

(b)

Figure 6-12. Wind directional dynamic response of the 30-story building: (a) peak story drift response; (b) peak base shear repose

Figure 6-13 represents the peak drift and floor acceleration responses over the building stories for different wind directions. It is shown that the overall peak drift ratio response is related to $27^{\text {th }}$ story, however, the peak floor acceleration is related to the roof level. The same trend of the decrease in the responses with changing the wind direction from $0^{\circ}$ to $45^{\circ}$ is shown in the figure. 


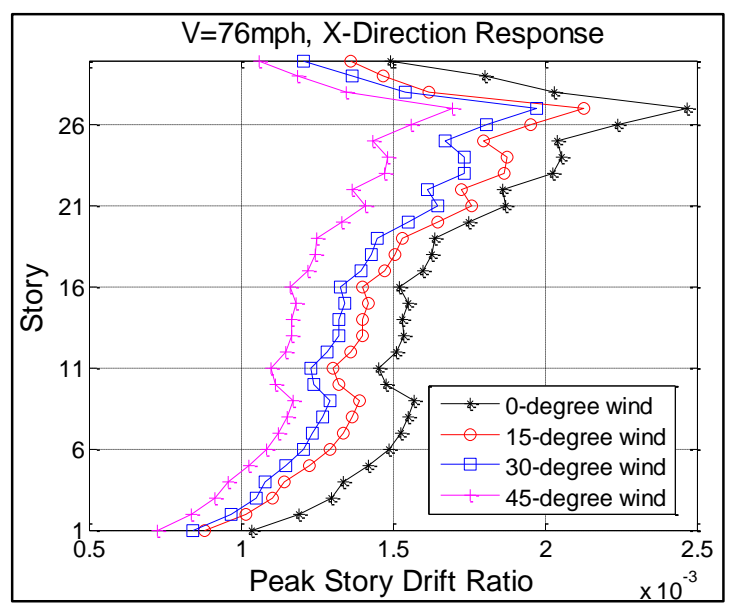

(a)

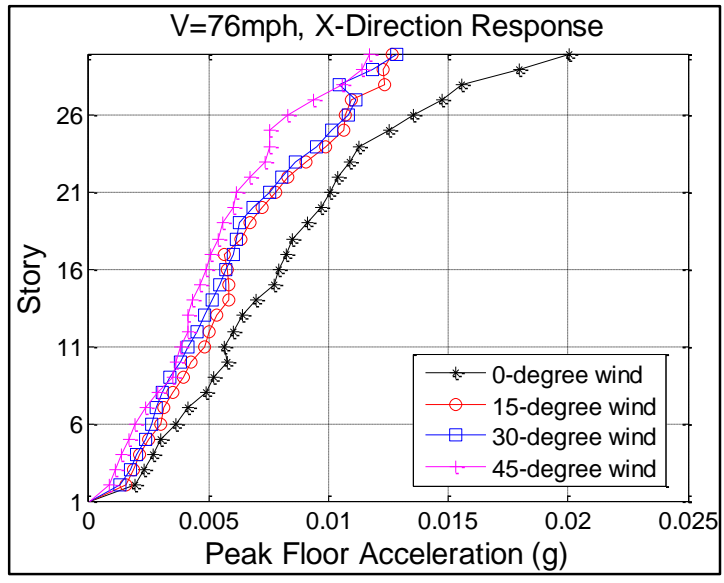

(c)

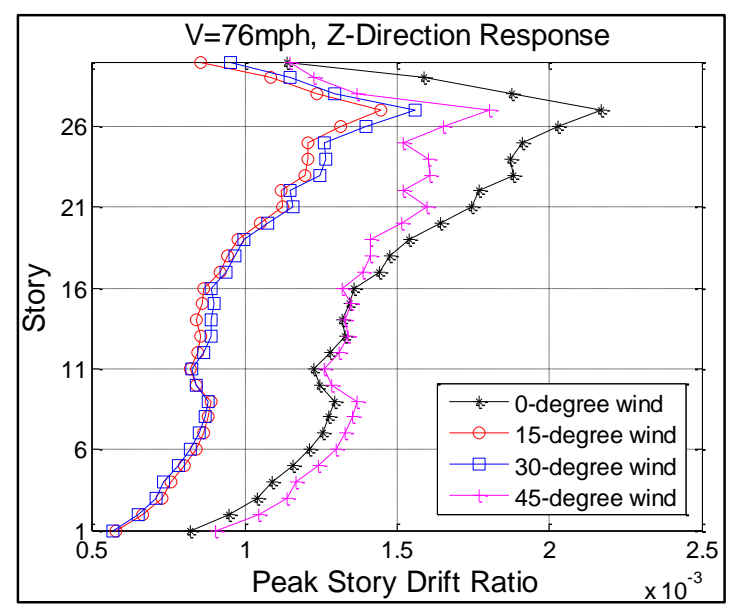

(b)

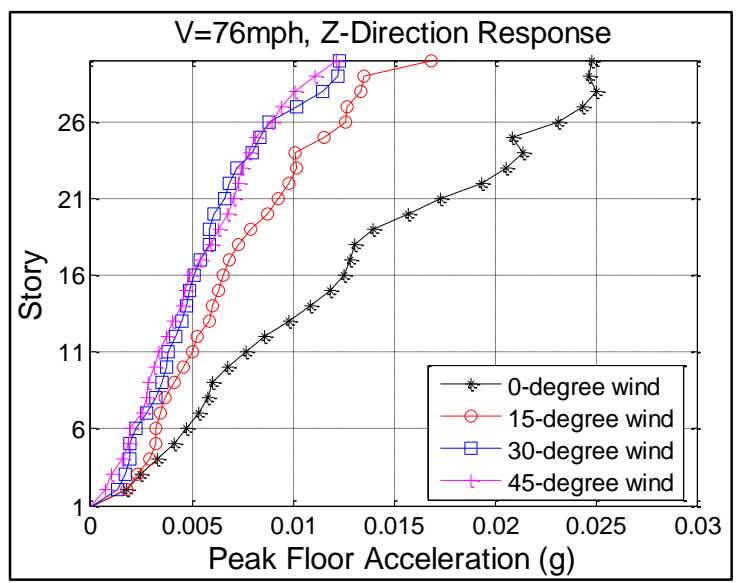

(d)

Figure 6-13. Wind directional dynamic response of the 30-story building: (a) peak drift story ratio

$X$-Direction; (b) peak drift story ratio in Z-Direction; (a) peak floor acceleration in X-Direction; (b) peak floor acceleration in Z-Direction

\subsubsection{Incremental Dynamic Analysis (Ida) Results}

For the 30-story building, incremental dynamic analyses were performed for 17 incremental levels of basic wind speeds. As already mentioned, the basic wind speed was chosen as the intensity measure in this study. The IDA was carried out by conducting wind nonlinear response history analyses with wind loads corresponding to basic wind speeds including 76, 90, 100, 105, $110,115,120,125,130,135,140,145,150,155,160,165$ and $170 \mathrm{mph}$. The IDAs were repeated for the wind angles of $0^{\circ}, 15^{\circ}, 30^{\circ}, 45^{\circ}$. As already explained for a symmetric square building 
and isolated wind tunnel data, the responses for the range of $0^{\circ}-45^{\circ}$ wind directions represent the responses for all wind directions around the building.

Figure 6-14 shows the IDA curves for peak story drift ratio and peak floor acceleration responses for all four wind directions. As shown in the figure, by changing the wind direction from zero to $45^{\circ}$ the responses decrease and the final collapse of the building occurs in accordance basic wind speed of $160 \mathrm{mph}$ in zero and $15^{\circ}$ wind directions, however for $30^{\circ}$ and $45^{\circ}$ wind directions the building was able to survive under the dynamic wind loads of $170 \mathrm{mph}$ basic wind speed. It is also shown that the larger floor acceleration is generally experienced in cross-wind direction. 


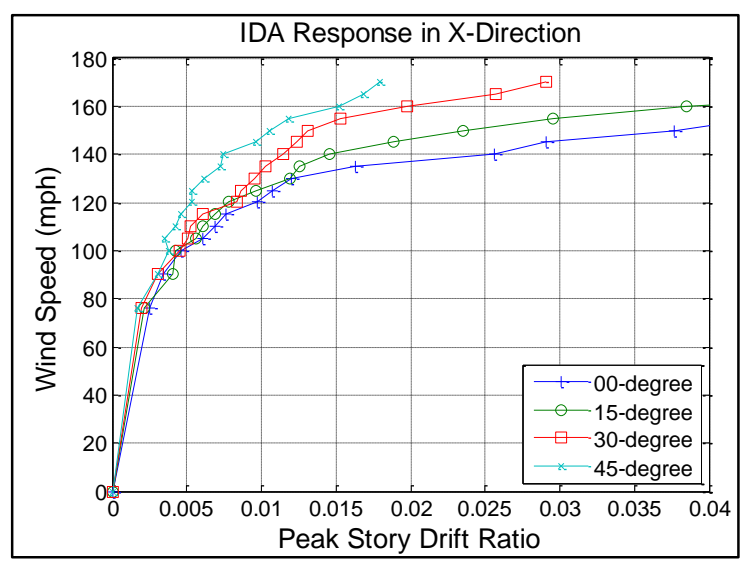

(a)

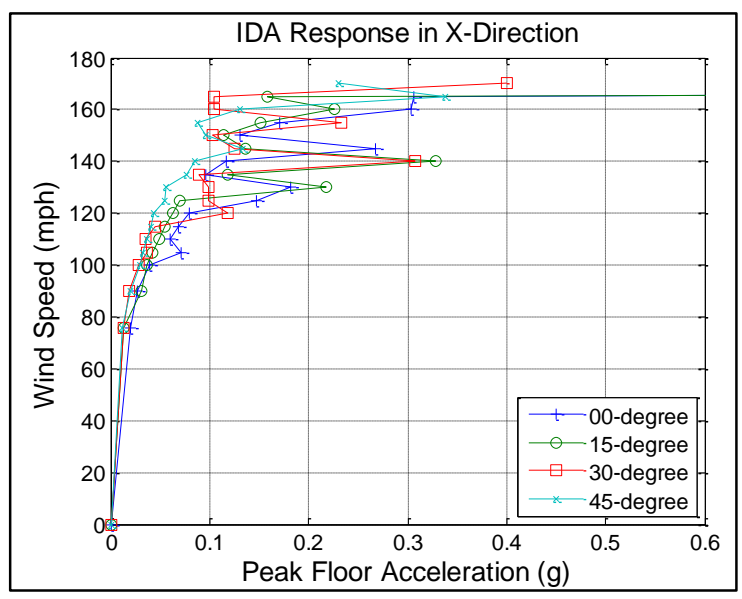

(c)

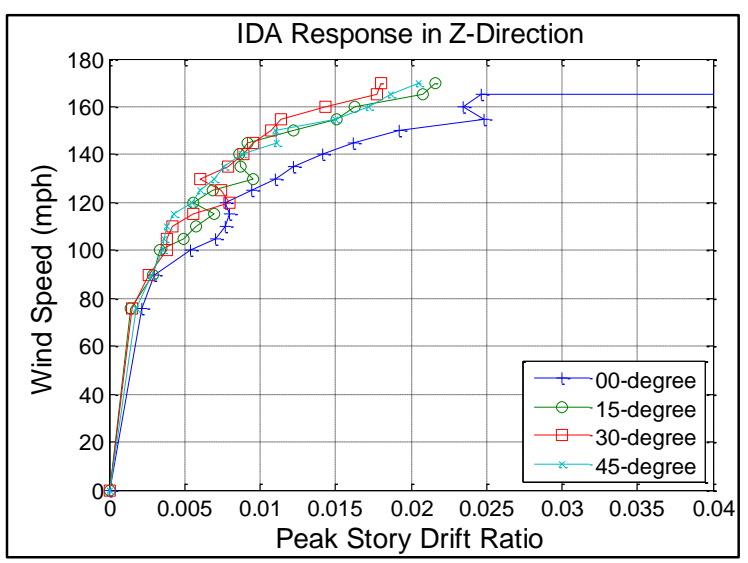

(b)

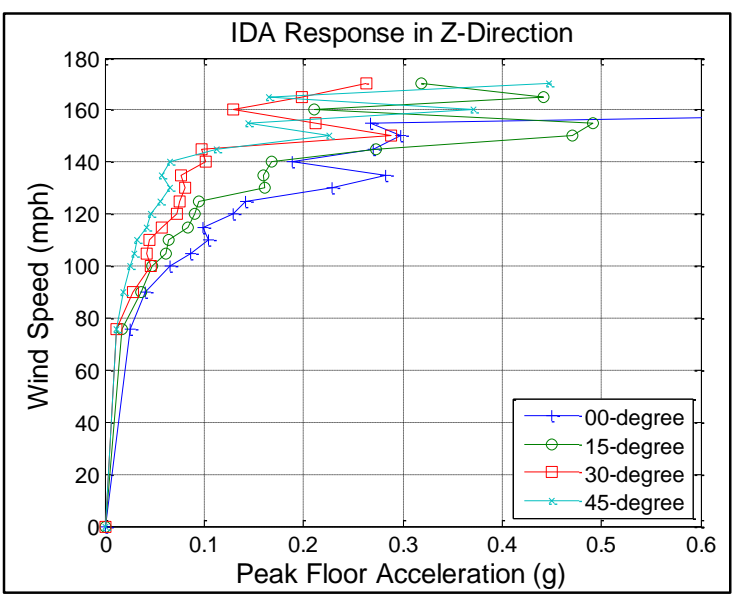

(d)

Figure 6-14. IDA responses of the 30-story building: (a) peak story drift ratio in X-direction; (b) peak story drift ratio in Z-direction; (c) peak floor acceleration in X-direction; (d) peak floor acceleration in Z-direction

\subsection{Analysis Results of The 40-Story Building}

\subsubsection{Modal Analysis Results}

The modal response of the 40-story building is shown in Figure 6-15. The figure shows the mode shapes, natural periods and type (either translational or torsional) of the first five modes of the building. It is shown that the same mode type sequence as for the two other high-rise buildings is obtained. 


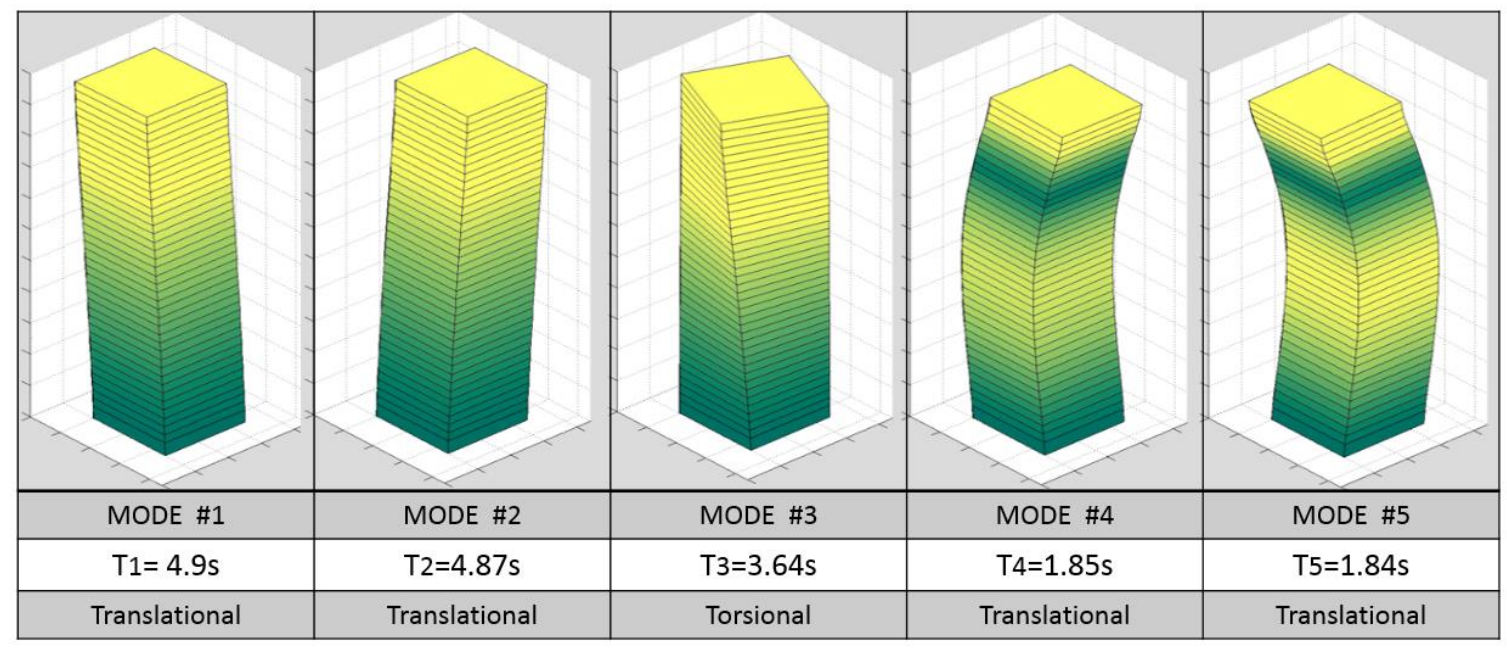

Figure 6-15. Modal Shapes, natural periods and the type of the first five modes of the 40-story building

\subsubsection{Nonlinear Static (Pushover) Analysis Results}

By subjecting the building model to gravity loads and the scaled incremental static wind loading, nonlinear static pushover wind analyses were carried out on the 40-story building in the two principal directions of the building. Figure 6-16a and 6-16b show the pushover curves obtained for two principal directions of the building, $\mathrm{X}$ and $\mathrm{Z}$ direction respectively. Figure 6-17 and Figure 6-18 illustrate the locations of plastic hinges for different types of nonlinearity in the pushover analyses in $\mathrm{X}$ and $\mathrm{Z}$ directions respectively. The same as for the 30-building model, extensive panel zone yielding is shown in both direction responses. Significant beam plastic hinging in lower levels up to 12 th story is also shown in both direction responses, while column plastic hinging is more significant in middle and upper levels. Remarkable nonlinearity in gravity connections is shown. It indicates a significant contribution of gravity framing in the lateral response of the building. Generally, as expected for a symmetric square building, the same responses and inelasticity destitutions are obtained for pushover in both directions. The wind design base shear according to ASCE 7-10, identical in both building direction, was equal to 3240 kips. As shown in Figure 5-16 the nonlinear response of the building resulted in the ultimate lateral resistance equal to 11940 kips identical for both $\mathrm{X}$ and $\mathrm{Z}$ direction responses. The wind 
over-strength in the two building major directions $\Omega_{\text {wind }}$ and $\Omega_{\text {wind }}$, were identically obtained equal to 3.5. Since the building design had been governed by the wind, the over-strength may reflect the remarkable lateral capacity of IMF high-rise buildings by allowing the nonlinearity of structural components as well as the contribution of the gravity framing.

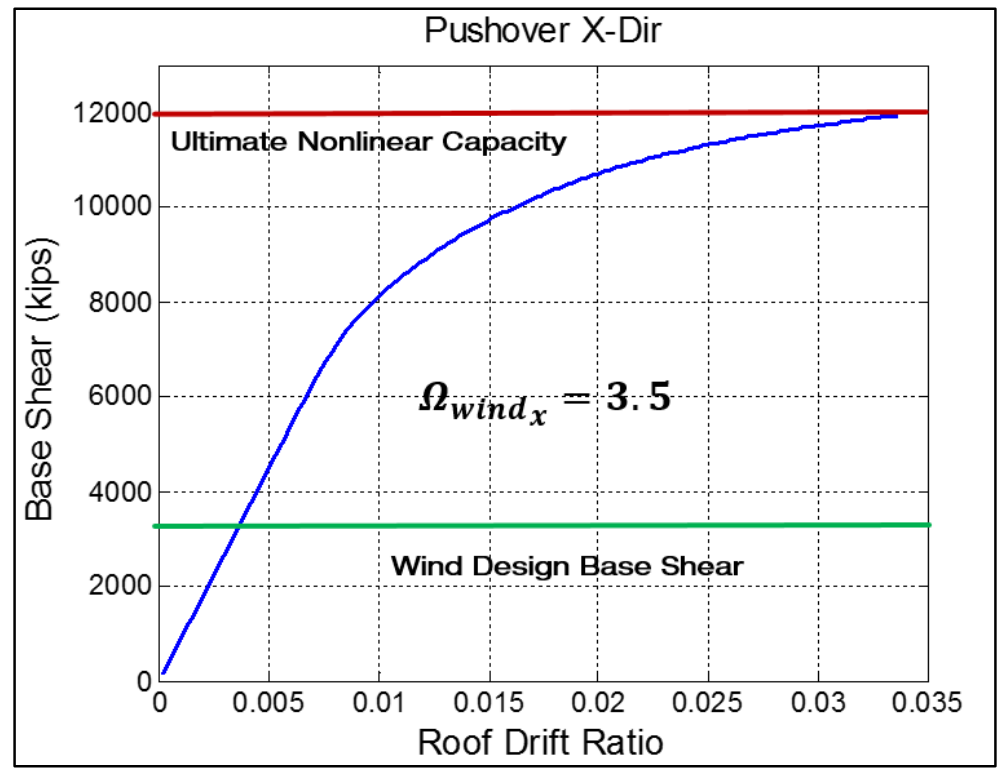

(a)

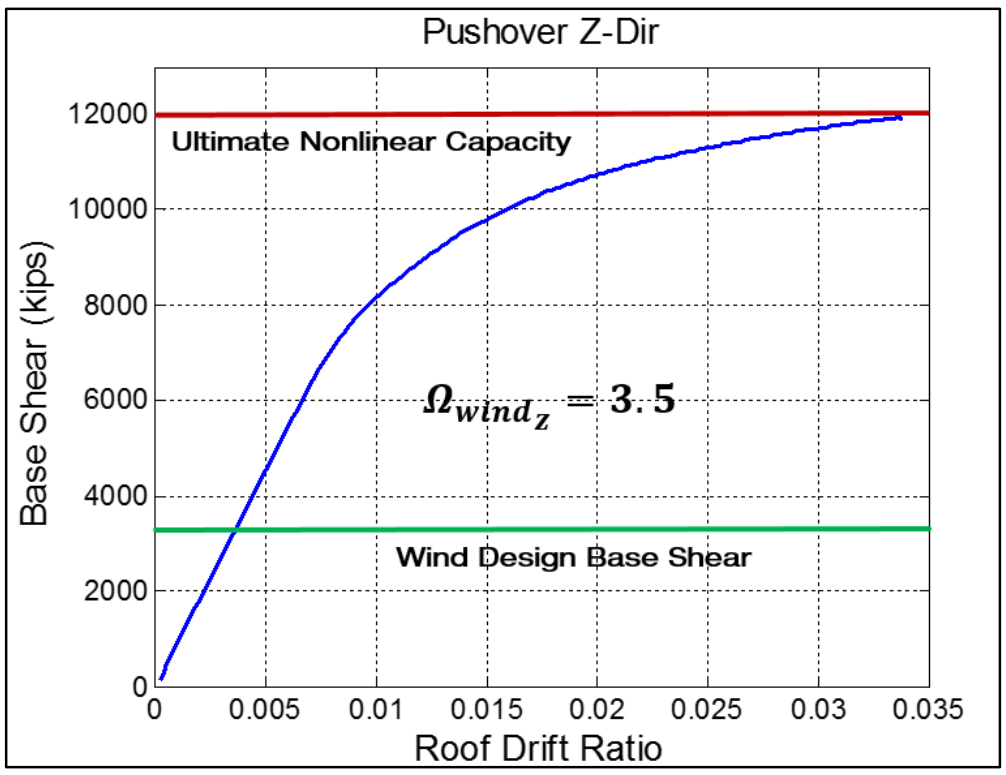

(b)

Figure 6-16. Wind pushover: (a) pushover curve in X-direction response; (b) Pushover curve in Z-direction response 


\section{- Panel Zone Plastic Hinge - Gravity Connection Plastic Hinge - Beam Plastic Hinge - Column Plastic Hinge}

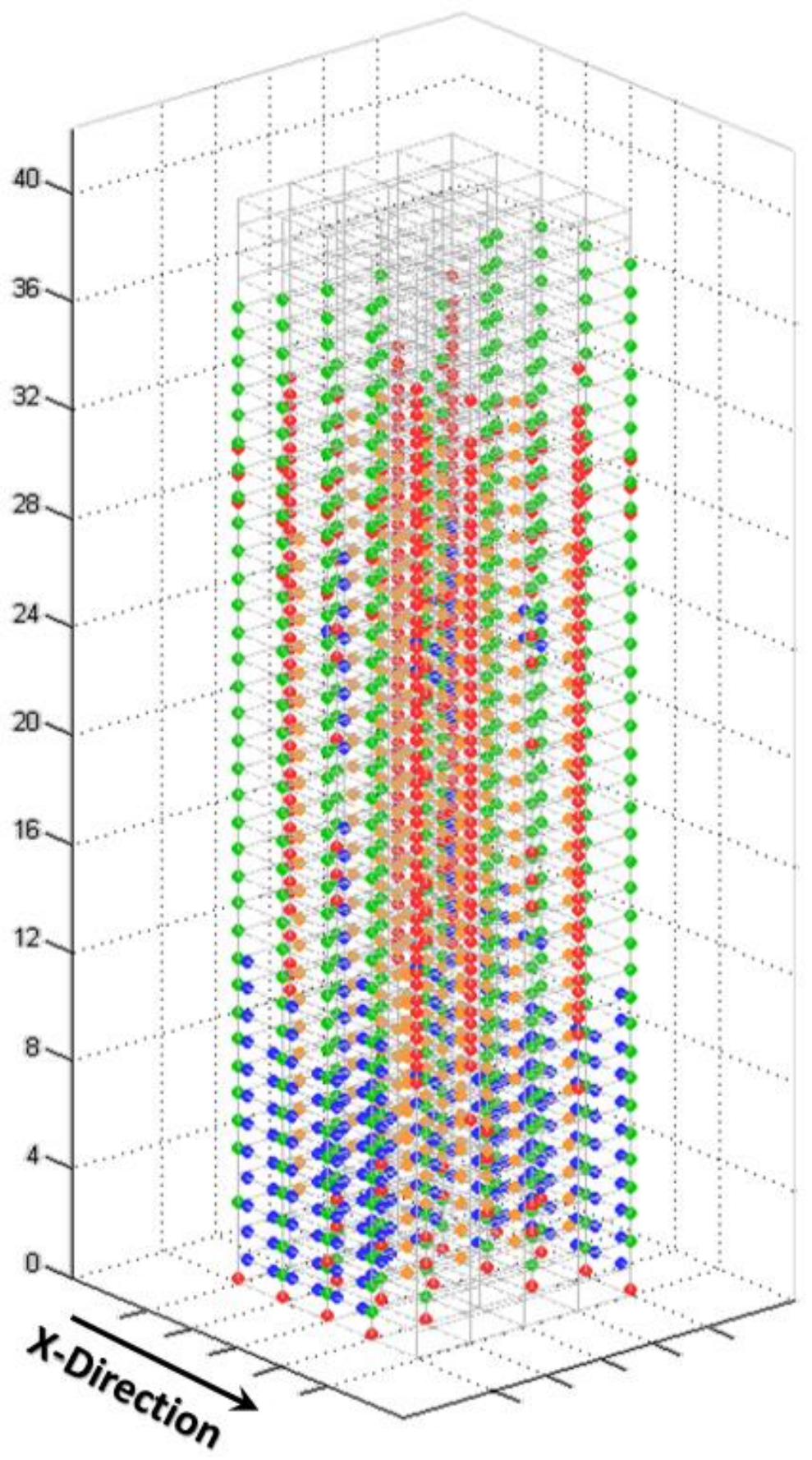

Figure 6-17. Wind pushover response of the 40-story building; nonlinearity distribution in nearcollapse stage in the $X$-direction response 
- Panel Zone Plastic Hinge - Gravity Connection Plastic Hinge

- Beam Plastic Hinge - Column Plastic Hinge

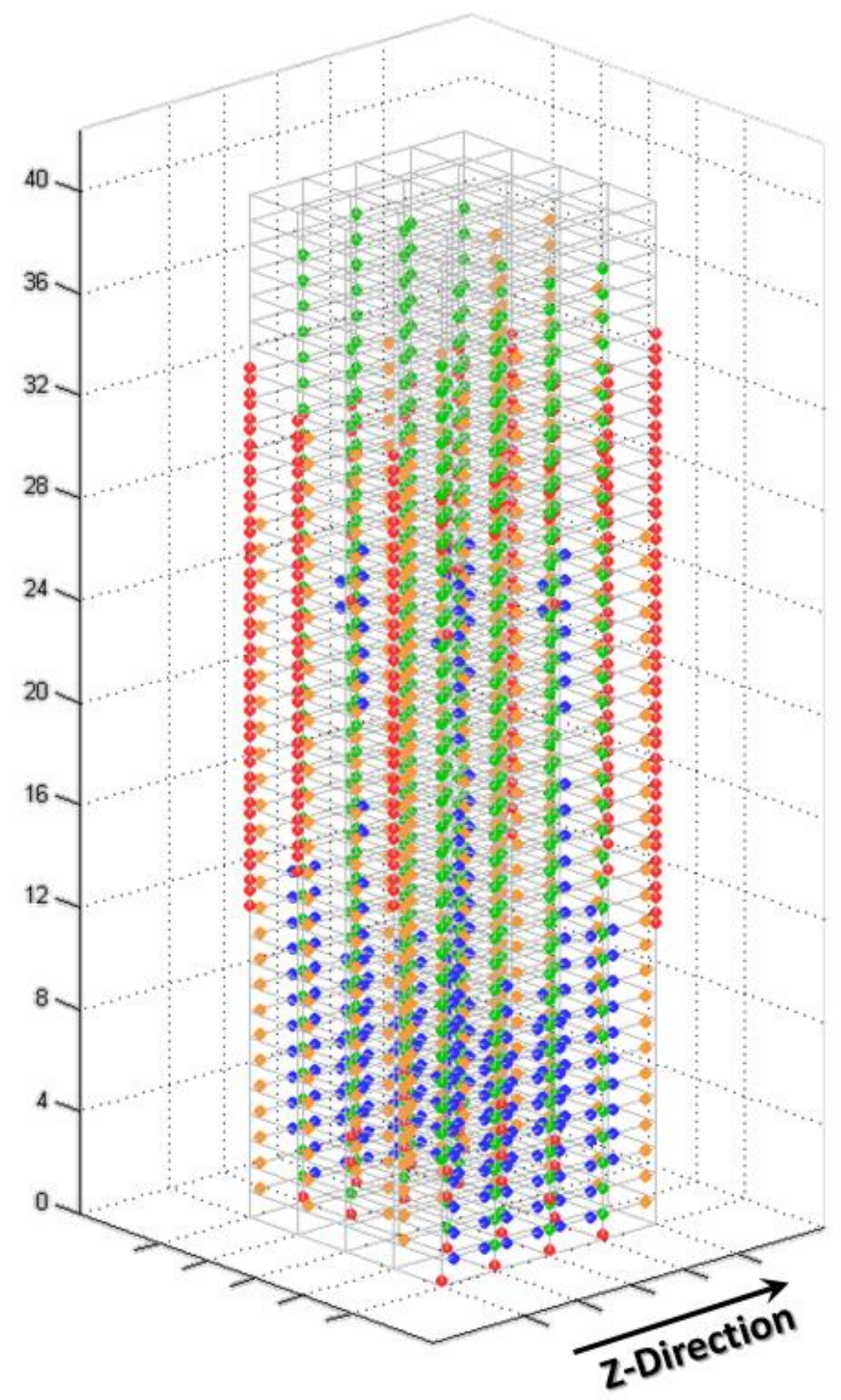

Figure 6-18. Wind pushover response of the 40-story building; nonlinearity distribution in nearcollapse stage in the Z-direction response 


\subsubsection{Nonlinear Response History Analysis Results}

Wind nonlinear history response analyses of the 40 -story building were conducted by applying dynamic wind loading simulated by 208 time-history point loads around the building by using the procedure described in previous chapter. The same as for two other high-rise building models, $2 \%$ critical damping ratio using Rayleigh damping were considered only for linear elements to avoid spurious damping forces.

The study of wind directionality of the dynamic wind response was conducted by plotting the wind responses for a constant wind speed of $76 \mathrm{mph}$ versus the wind directions as shown Figure 6-19 and Figure 6-20. The figures show a general decreasing trend in responses by increasing the wind angle from $0^{\circ}$ to 45 .

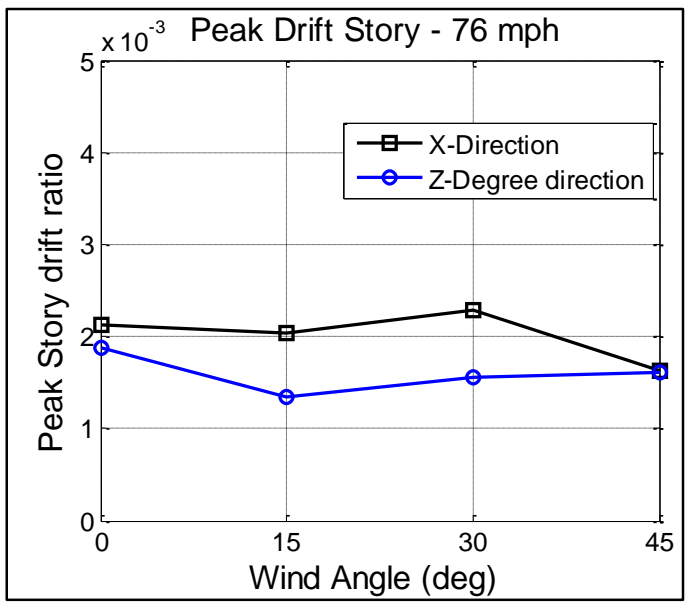

(a)

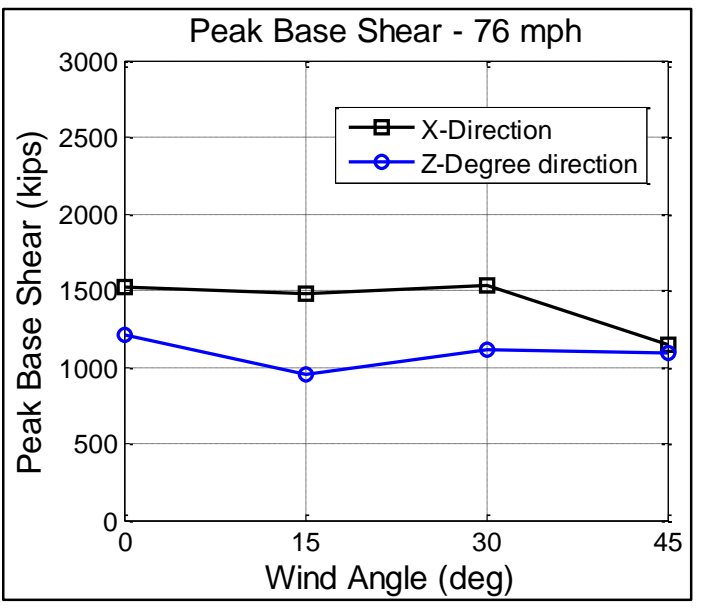

(b)

Figure 6-19. Wind directional dynamic response of the 40-story building: (a) peak drift story ratio; (b) peak base shear

Peak story drift and peak floor acceleration responses over the building's height are shown in Figure 6-20. It is shown that the peak drift ratio response corresponds to the $25^{\text {th }}$ story, however, the peak floor acceleration is related to the roof level. 


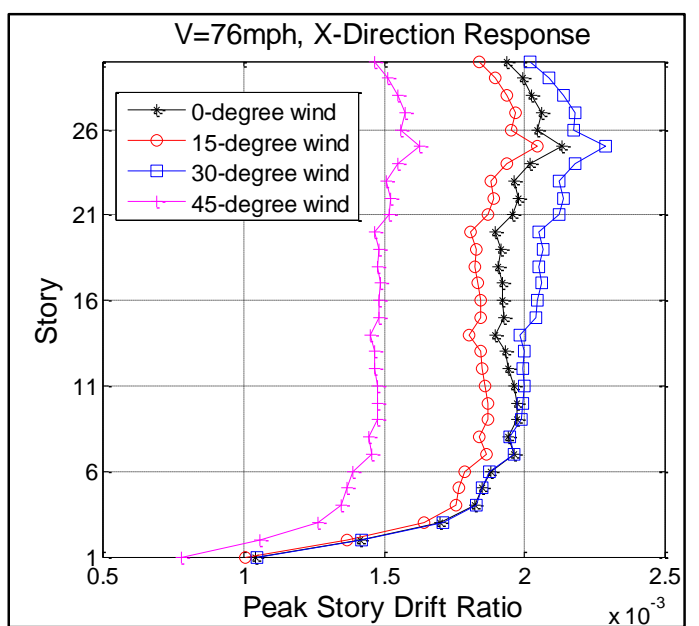

(a)

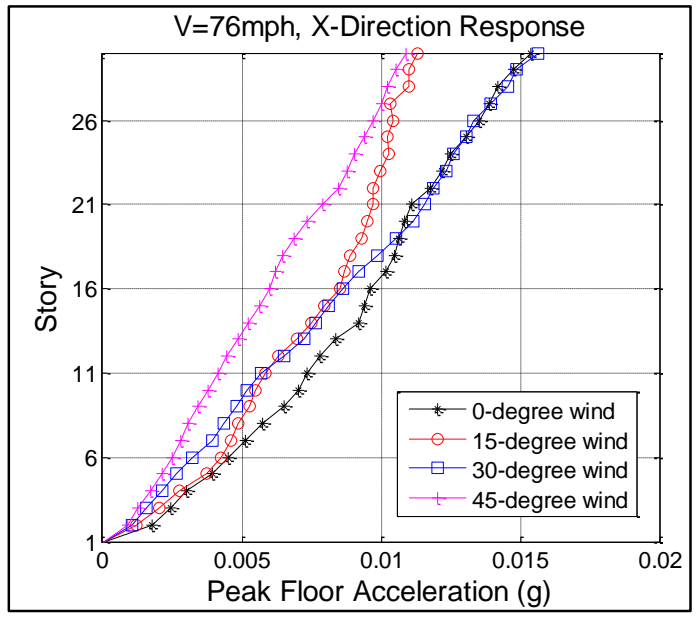

(c)

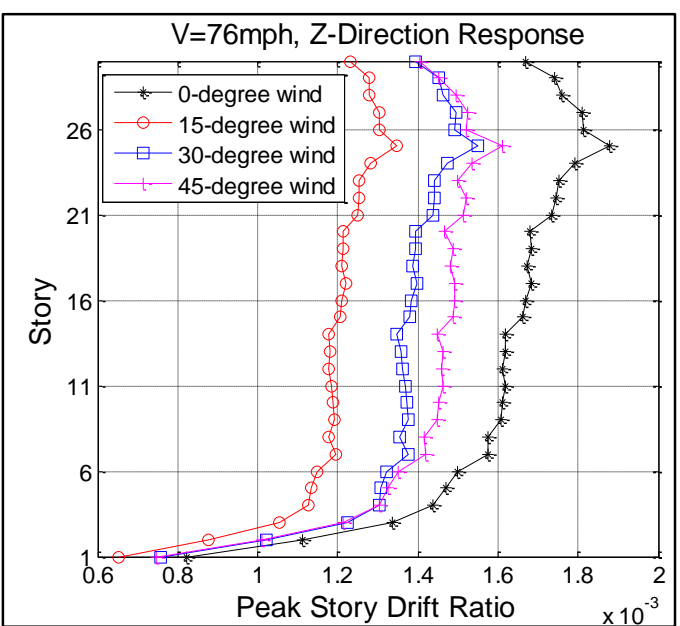

(b)

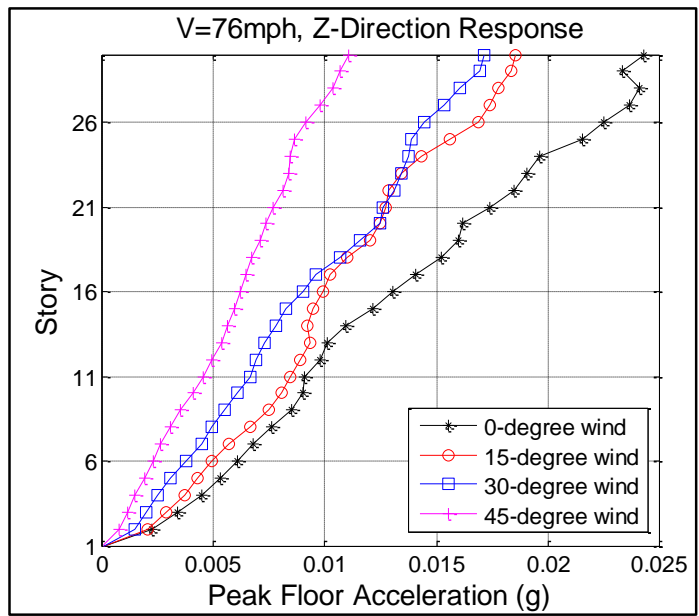

(d)

Figure 6-20. Wind directional dynamic story-response of the 40-story building: (a) peak drift story ratio in X-direction; (b) peak drift story ratio in Z-direction; (a) peak floor acceleration in $X$-direction; $(b)$ peak floor acceleration in Z-direction

\subsubsection{Incremental Dynamic Analysis (Ida) Results}

For the 40-story building, the incremental dynamic analyses were performed for 17 levels of wind speeds for varied wind directions. The IDA in each wind direction was carried out by conducting wind nonlinear response history analyses with wind loads corresponding basic wind speeds including $76,90,100,105,110,115,120,125,130,135,140,145,150,155,160,165$ and 170 mph. The IDAs were repeated for the wind angles of $0^{\circ}, 15^{\circ}, 30^{\circ}, 45^{\circ}$. 
IDA curves of peak story drift ratio and peak floor acceleration responses in all four wind directions are shown in Figure 6-21. As shown in the figure, the building collapse corresponds to wind speed of $150 \mathrm{mph}$ represented by a large inter-story drift in an along-wind direction (Xdirection). The same as observed for two other buildings larger floor acceleration response is experienced in a cross-wind direction (Z-direction).

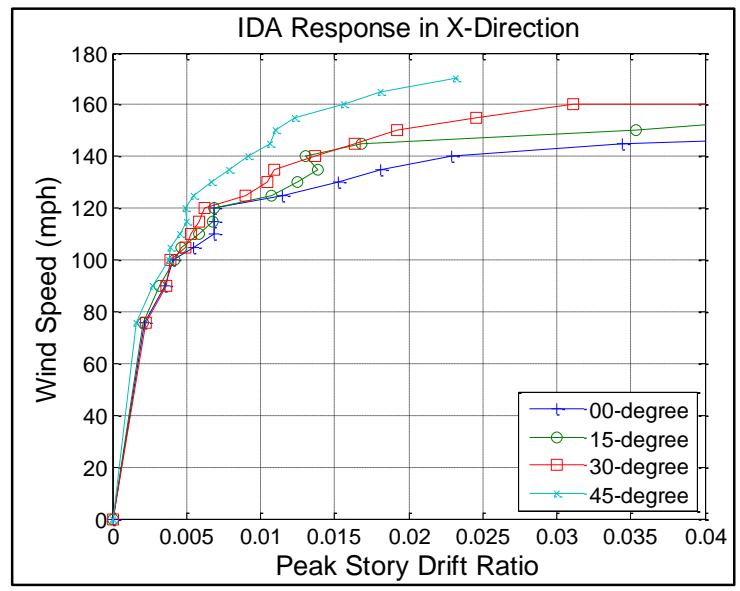

(a)

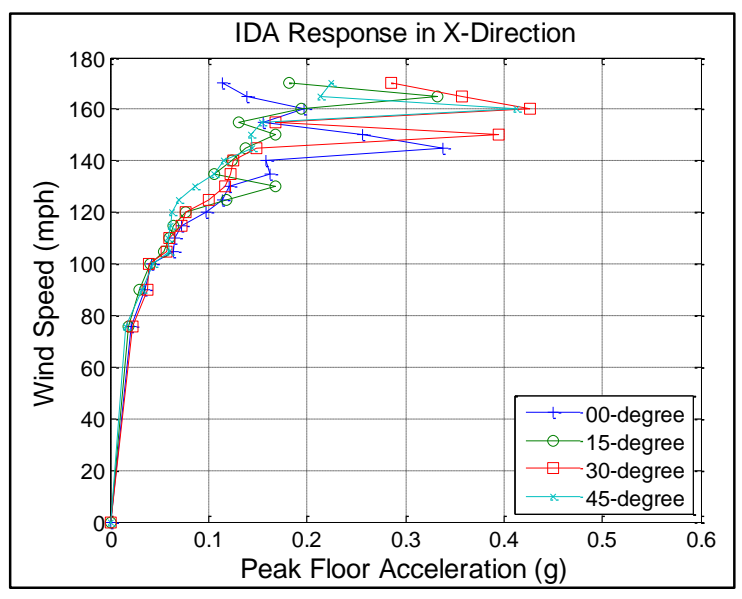

(c)

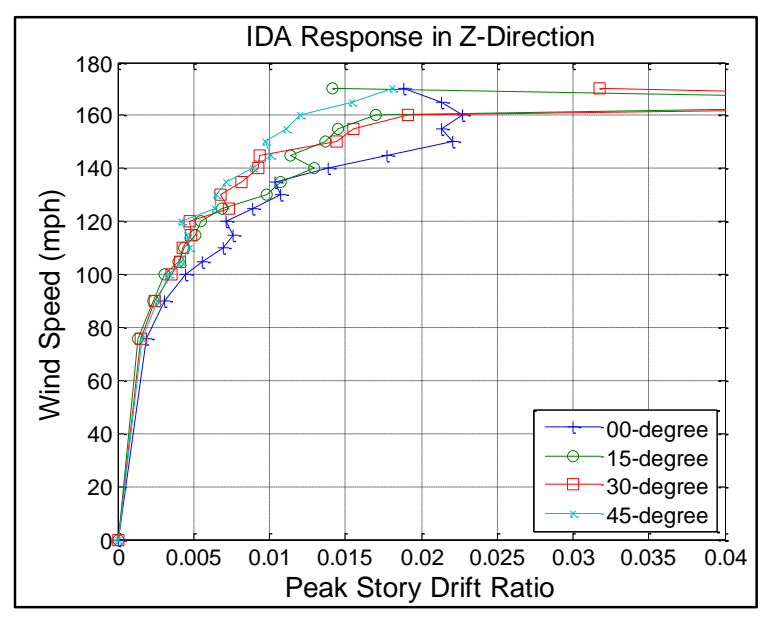

(b)

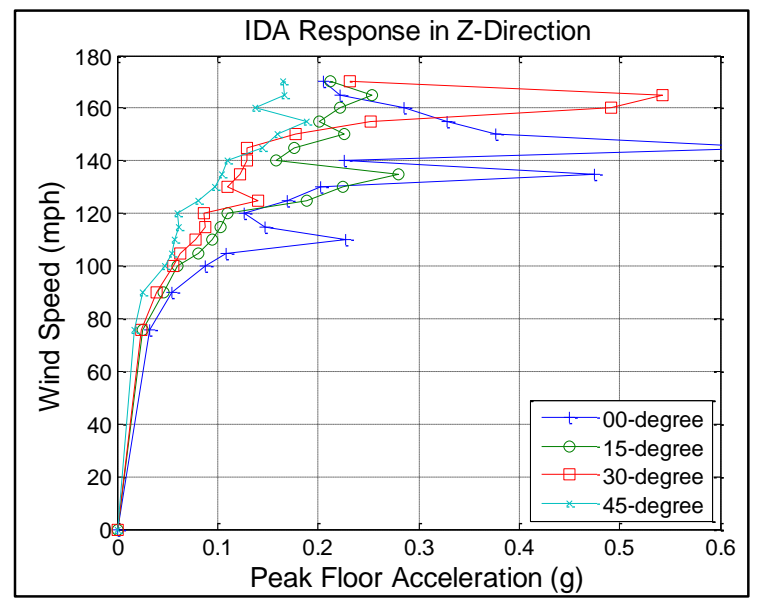

(d)

Figure 6-21. IDA responses of the 40-story building: (a)peak story drift ratio in X-direction; (b) peak story drift ratio in Z-direction; (c) peak floor acceleration in X-direction; (d) peak floor acceleration in Z-direction 


\section{CHAPTER VII}

\section{DEVELOPMENT AND IMPLANTATION OF A WIND PERFORMANCE ASSESSMENT APPROACH}

\subsection{Introduction}

Development and practical implementation of a wind performance-based evaluation approach for high-rise buildings are the major objectives of this study. A wind performance assessment approach is to reflect the functionality and resilience of buildings for different levels of wind hazard by developing a set of engineering metrics and indicators representing the building's status in both component and global response levels. Generally, conducting the wind performance assessment requires a comprehensive understanding of the wind responses of the building to the different levels of wind hazard and an engineering framework introducing the different types of the building performances concerned, performance levels and the corresponding acceptance criteria. The framework needs to consider different types of performances representing different aspects of the functionality and resilience of high-rise buildings during wind events. Similar to the seismic performance based evaluation approach, the framework is to require a set of performance objectives related to several discrete hazard levels (depending on the building's type) where by meeting the performance requirements it promises acceptable functionality and resilience of the building during extreme wind events. The specified performances need to be properly correlated to structural outputs known as "Engineering Demand Parameters (EPDs)" to reflect the performances status of the building. Performance levels and associated acceptance criteria need to be defined to present the status of the building with respect to a specific performance in terms of discrete performance levels set according to associated damage and money loss of retrofitting. 
In practice, by conducting wind IDAs, comprehensive data representing the wind responses of the high-rise building is obtained. Using the IDA results along with a framework, relating the engineering parameters to performance indicators and introducing associated performance levels and acceptance criteria, will provide a comprehensive performance assessment of the high-rise building subjected to wind loads. This chapter presents the development of a wind performancebased evaluation approach by using IDA results in conjunction with a developed performancesbased framework partially adopted from available state-of-the-art wind performance criteria. This chapter also presents the implementation of the approach to all the three high-rise building and discusses the estimated performances.

\subsection{Development of The Wind Performance Assessment Approach}

In this study, a wind performance assessment approach is developed based on using wind IDA results in conjunction with a wind performance-based framework developed in accordance with the wind performance assessment criteria available in the literature. The proposed wind performance-based engineering framework generally considers four performance levels: (1) Fully Operational (FO); (2) Limited Operations (LO); (3) Life Safety (LS) and (4) Collapse Prevention (CP) as described in Table 7-1. The table explains each performance level with its corresponding level of functionality interruption and damage to the building. The table also relates a wind hazard range in terms of Mean Recurrence Interval (MRI) to each performance objective. Correspondingly, in order to undertake a wind performance based design/evaluation the framework requires the building to meet FO, LO, LS and CP performance objectives for windstorm and hurricanes events with 1-10 years, 50-100 years, 700-1700 years and 10,000 years return period respectively. 
Table 7-1. Proposed performance levels

\begin{tabular}{|c|c|c|c|c|}
\hline \multicolumn{5}{|c|}{ PERFORMANCE LEVELS } \\
\hline BUILDING ELEMENT & $\begin{array}{c}\text { COLLAPSE } \\
\text { PREVENTION (CP) }\end{array}$ & LIFE SAFETY (LS) & LIMITED OPERATIONS (LO) & FULLY OPERATIONAL (FO) \\
\hline Storm Level (MRI Range) & $10,000 \mathrm{Yr}$ & $700-1700 \mathrm{Yr}$ & $50-100 \mathrm{Yr}$ & $1-10 \mathrm{Yr}$ \\
\hline $\begin{array}{l}\text { GENERAL OVERALL } \\
\text { BUILDING PERFORMANCE }\end{array}$ & $\begin{array}{l}\text { Building } \\
\text { survives but } \\
\text { unrepairable }\end{array}$ & $\begin{array}{l}\text { Repair likely required } \\
\text { before occupancy } \\
\text { - } \text { Significant cost and time } \\
\text { to repair } \\
\text { - Access \& egress } \\
\text { compromised but } \\
\text { generally functional } \\
\text { Relocation of occupants } \\
\text { likely req'd within building } \\
\text { - Severe level of occupant } \\
\text { discomfort expected }\end{array}$ & $\begin{array}{l}\text { - Building repairs minor } \\
\text { - } \text { Minor disruption to } \\
\text { access \& egress } \\
\text { Immediate re- } \\
\text { occupancy expected } \\
\text { Significant occupant } \\
\text { discomfort during storm } \\
\text { event } \\
\text { - All MEP \& other sytems } \\
\text { repairable }\end{array}$ & $\begin{array}{l}\text { - } \quad \text { No water infiltration } \\
\text { normal operational remain } \\
\text { functional } \\
\text { - Access \& egress unaffected } \\
\text { - } \quad \text { Continuous occupancy \& use } \\
\text { of building expected } \\
\text { - Minor effect to occupant } \\
\text { comfort }\end{array}$ \\
\hline
\end{tabular}

The proposed approach considers three type of performance of high-rise buildings subjected to wind loading reflecting the strength and functionality status of building for different levels of wind hazard. Three performances considered in this approach can be divided into two levels of (a) component level response and (b) global level response performances. The three types of performance considered in this study include: (1) structural component performance, (2) cladding performance to wind-induced shear deformation; and (3) serviceability motion comfort performance. Among them, the first one is related to component level response, and the two latter are related to global level response. The procedure of wind performance assessment for evaluating these three performances including introducing the performances levels and the corresponding acceptance criteria are explained in following sections.

\subsubsection{Structural Component Performance}

The first type of performance considered in this approach is the structural component performance which reflects the level of force/deformation demand developed in structural components due to dynamic wind response in respect to force/deformation capacity. The level of the nonlinearity developed in the structural components correlates with the damage to structural the frame, the corresponding functionality interruption and the consequent rehabilitation costs. For the wind performance based assessment, component performance levels are set to reflect different stages of damage to the structural system including: no damage, minor damage, 
significant damage and extensive damage (unrepairable) respectively for FO, LO, LS and CP performance levels as shown in Table 7-1.

For component response performance, the acceptance criteria were adopted from the wind performance-based design framework suggested by Griffis et al. (2012). The framework considers three component response performance levels including: (1) Continued Occupancy (CO); (2) Operational (Op); and (3) Limited Interruption (LI) in accordance with the level of force/deformation developed as shown in Figure 7-1a, for both deformation-controlled and forcecontrolled components. While the criteria are suggested in accordance with the ASCE 7-10 nonlinear component force- deformation relationship model, it is adopted and fitted to the MIK model used in this study. Accordingly, the acceptance criteria of the three performance levels of CO, Op and LI were adopted respectively for FO, LO and LS performance levels. Figure 7-1b shows the performances and corresponding acceptance criteria developed for different types of structural components used in this study. The figure shows the acceptance criteria considered for the CP performance level in accordance with the capping strength/deformation for displacementcontrolled components.

For component performance assessment, in each hazard intensity level which is represented by the corresponding basic wind speed, the dynamic responses of all structural components are evaluated in accordance with acceptance criteria shown in Figure 7-1b. Moving to next performance level was considered when at least $10 \%$ of the same component type passed the performance limit. 

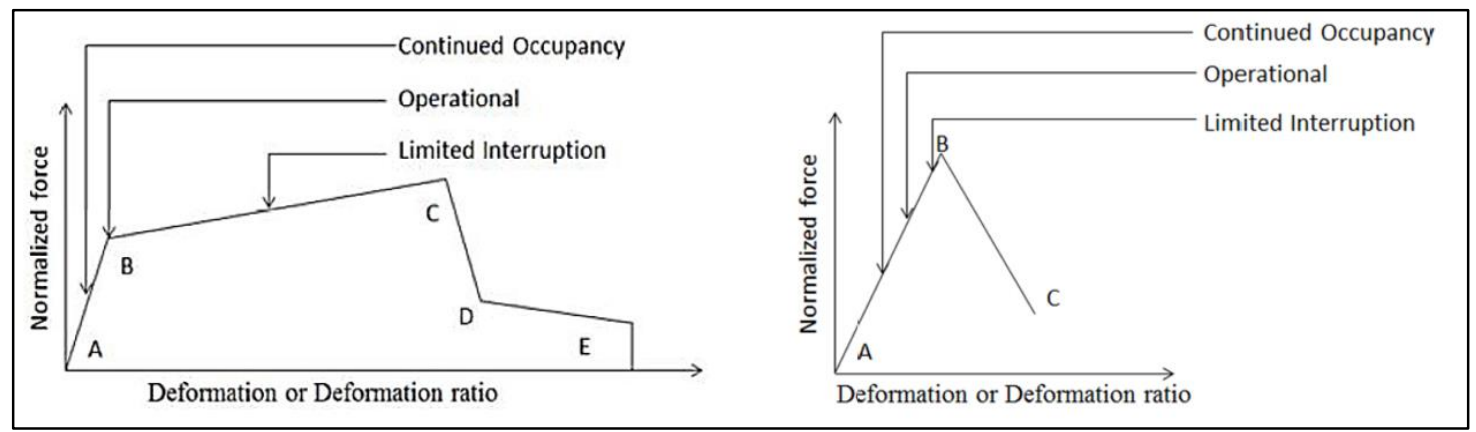

(a)

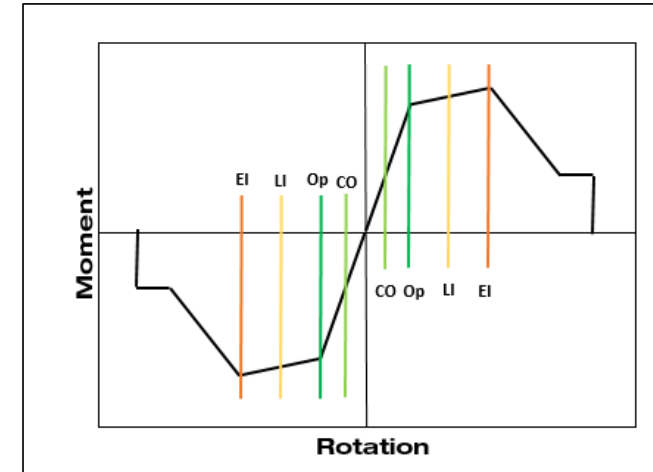

Steel Beam and Column Elements

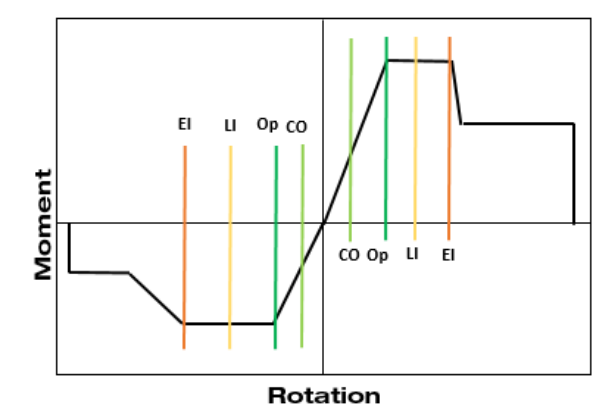

Gravity Connections

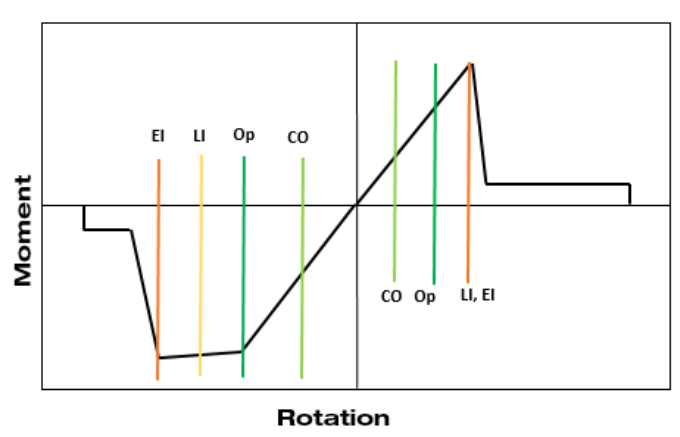

Pre-Northridge Connections

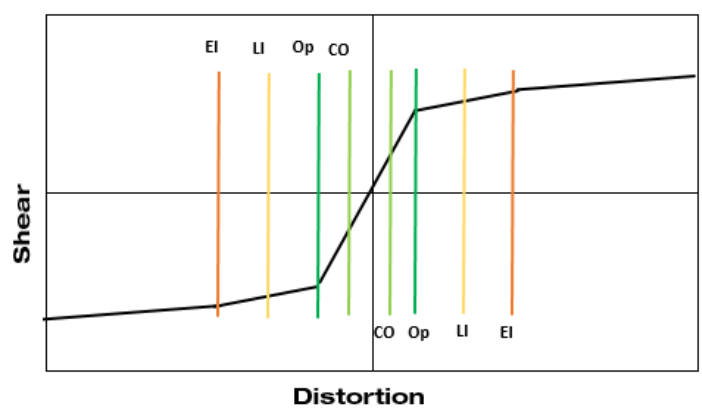

Panel Zone

(b)

Figure 7-1. Component acceptance criteria: (a) recommended by Griffis et al. (2012); (b) adopted and used in this study

Two additional performance types evaluated in associated with building's global response are: (1) performance of cladding system to large shear deformation; and (2) serviceability performance for motion comfort. The approach to assessing these performances is explained in following sections. 


\subsubsection{Cladding Performance to Wind-Induced Shear Deformation}

The cladding is one of the major concerns in the response of high-rise buildings to extreme wind excitations. The performance of high-rise buildings in major recent hurricanes indicates significant damage to cladding mainly occurred due to wind-borne debris. However, by allowing nonlinearity in the structural components larger shear deformation will be expected. This large shear distortion in interaction with cladding system may also result in significant damage to the cladding. This concern necessitates considering the performance of cladding to shear deformation in the wind performance assessment approach. Certainly, other important types of performance related to the cladding response to extreme wind events (such as damage due to wind-borne debris and local overload due to excessive local pressure) need to be considered which requires profound research about current claddings and it is beyond the scope of this research.

In this wind performance assessment approach, the method suggested by Griffis (2003) and Aswegan et al (2015) was adopted to evaluate the damage to the cladding system due to lateral deformation of the building. In this method, the wind-induced shear deformation for a given cladding or partition panel called Drift Damage Zone (DDZ) is evaluated by a measure called Deformation Damage Index (DDI). Figure 7-2 presents the estimated DDI for a given DDZ in the $\mathrm{n}^{\text {th }}$ story. It is shown that the equation incorporates the vertical and horizontal displacements of all four corners to estimate the precise shear distortion acting on the given cladding or partition panel. 


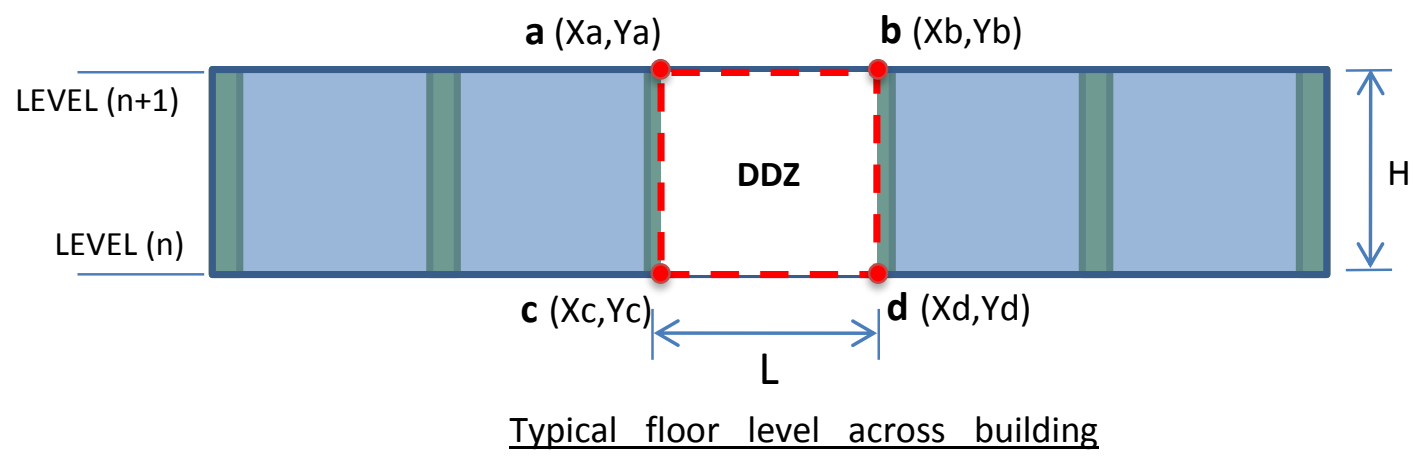

$$
\begin{aligned}
& \text { D1 }=(X a-X c) / H, D 2=(X b-X d) / H \\
& \text { D3 }=(Y d-Y c) / L, \quad D 4=(Y b-Y a) / L \\
& \mathrm{DDI}=0.5 \times(\mathrm{D} 1+\mathrm{D} 2+\mathrm{D} 3+\mathrm{D} 4)
\end{aligned}
$$

Figure 7-2. Definition of Deformation Damage Index (DDI) for a given Drift Damage Zone (DDZ) in $n^{\text {th }}$ story

In the current approach, the acceptance criteria for cladding response performances were adopted from the framework suggested by Griffis et al. (2012). Regarding curtain wall cladding systems, the framework suggests acceptance criteria as follows: (1) H/400 shear deformation for $\mathrm{CO}$ performance level; (2) H/220 shear deformation for Op performance level; and (3) H/140 shear deformation for LI performance level, where $\mathrm{H}$ is the story height. With respect to performance levels introduced in this study, the following acceptance criteria were considered: (1) H/400 shear deformation for FO performance level; (2) H/220 shear deformation for LO performance level; and (3) H/140 shear deformation for LS. The approach does not consider the CP performance level for cladding, for a structurally unrepairable building the damage to the cladding is most probably not critical.

\subsubsection{Serviceability Motion Comfort Performance}

The dynamic nature of the wind loads in conjunction with the flexibility of high-rise buildings may pose serious serviceability issues by causing discomfort to occupants during the wind induced motion. For today's tall buildings, the minimum building load criteria of ASCE 7 are usually supplemented by additional design checks to control building motions in order to 
maintain human comfort under less extreme winds that occur more frequently than the design wind loads. These criteria vary depending on the building occupancy, but generally limit the maximum building accelerations to less than about 10 to 25 milli-g's for wind speeds with 1 - to 10-year return periods. Wind serviceability performance assessment allows a comprehensive estimation of the serviceability status of the building under different levels of wind hazard to be undertaken.

In the current approach, a serviceability motion comfort performance assessment has been developed by adopting the performance limits suggested by Chang (1973) presented in Table 7-2 , perception ranges found in practice presented in Table 7-3, and the ISO provisions (ISO, 2004) shown in Figure 7-3. The two earlier comfort criteria suggest motion discomfort is associated with induced wind acceleration without regard to frequency of motion. However, ISO provides motion comfort threshold curves for residential and office buildings as a function of building's natural frequency. ISO requires the criteria for wind response for wind-induced vibrations to be based on 1-year MRI wind.

Estimating the building's serviceability status over the full range of wind hazard as suggested in this approach is critical for high-rise buildings which are supposed to stay operational or accommodate people during hurricanes (shelter-in-place buildings). Appropriate performance objectives need to be considered in accordance with the expected functionality to assure acceptable performance of the buildings during extreme wind events. 
Table 7-2. Serviceability performance criteria related to motion comfort (Chang, 1973)

\begin{tabular}{|c|c|}
\hline Peak Floor Acceleration & Comfort Limit \\
\hline$<5 \mathrm{mg}$ & Not Perceptible \\
\hline $5-15 \mathrm{mg}$ & Threshold of Perceptibility \\
\hline $15-50 \mathrm{mg}$ & Annoying \\
\hline $50-150 \mathrm{mg}$ & Very Annoying \\
\hline$>150 \mathrm{mg}$ & Intolerable \\
\hline
\end{tabular}

Table 7-3. Suggested serviceability criteria related to motion comfort

\begin{tabular}{|c|c|}
\hline Peak Acceleration Level & Perception Description \\
\hline$<5 \mathrm{mg}$ & Imperceptible to most occupants \\
\hline $5-15 \mathrm{mg}$ & Perceptible range to most occupants \\
\hline $20-25 \mathrm{mg}$ & Target range for office building occupancy \\
\hline$>28 \mathrm{mg}$ & Annoying range for most occupants \\
\hline$>40 \mathrm{mg}$ & Very annoying and difficult walking for most occupants \\
\hline
\end{tabular}

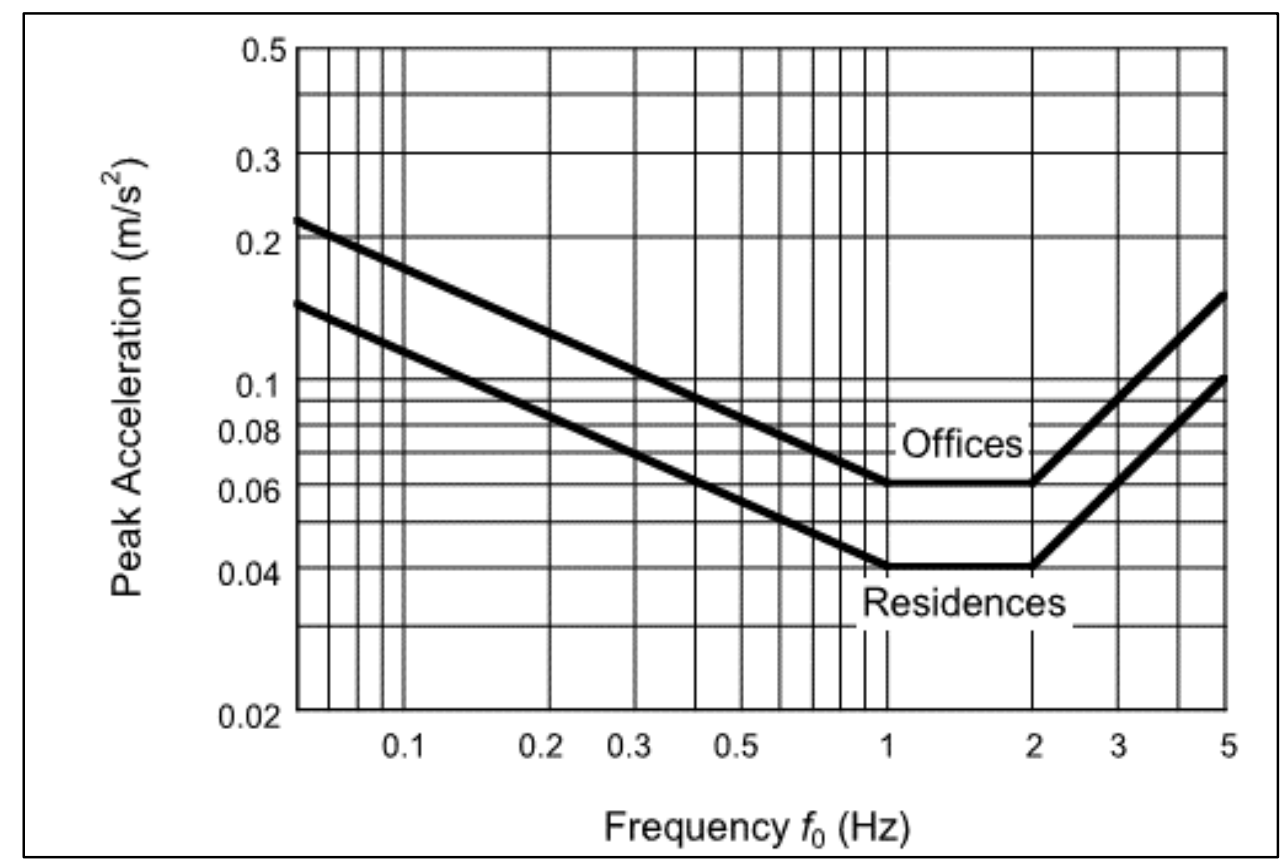

Figure 7-3. Basic evaluation curves for wind-induced vibration (ISO 10137 (2004), Figure D.1) 


\subsection{Wind Performance Assessment of the Case Study Buildings}

A wind performance-based approach practically was implemented to assess the performance of three case study buildings. Using the IDA results and the assessment approach explained in the previous section, the three buildings were evaluated for three type of wind performance including: structural components, cladding response to shear deformation and serviceability motion comfort response. The following sections describe the evaluations and the estimated performances.

\subsubsection{Wind Performance Assessment of The 47-Story Building}

\section{Structural Component Performance}

Wind performance assessment of the structural components was conducted by evaluating the responses of all existing components with respect to the introduced acceptance criteria. Figure 7-4 to Figure 7-6 show the performance status for all structural components resulting from IDAs for all six critical wind directions. In the figures, the black thick line is the performance envelope which represents the expected performance level for the entire building for that specific wind direction. For example, for $180^{\circ}$ wind direction, it is shown that at $76 \mathrm{mph}$ wind speed the component performance changes from Fully Operational (FO) to Limited Operations (LO). Maintaining the LO performance level from $76 \mathrm{mph}$ to $82 \mathrm{mph}$, the component performance level changes to Life Safety (LS) level at $86 \mathrm{mph}$ basic wind speed. It remains at the same performance level until the building collapse level corresponding to $136 \mathrm{mph}$ where it finally exceeds to Collapse Prevention (CP) criterion. 

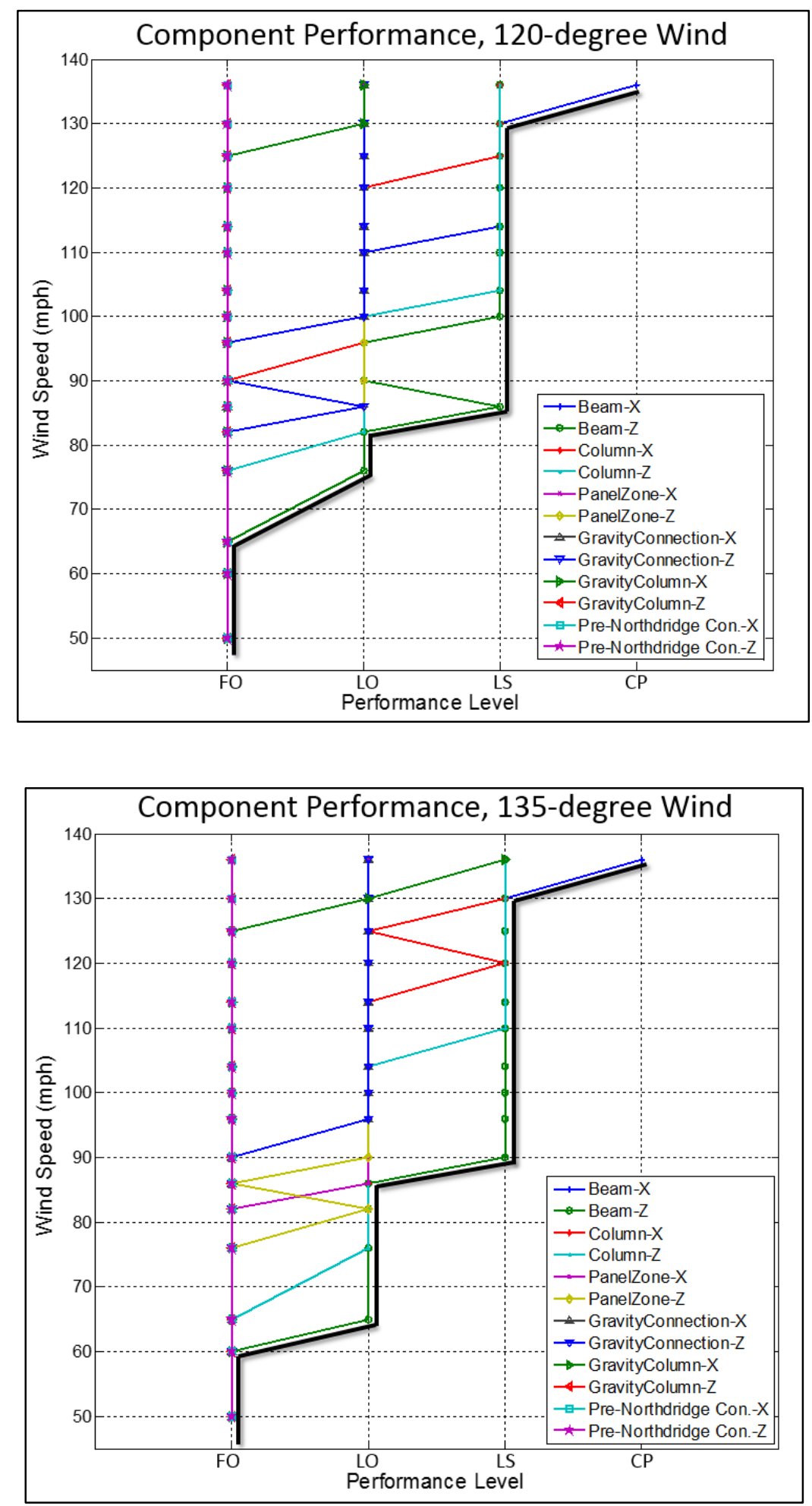

Figure 7-4. Component performance assessment of the 47-sotry building for 120 and 135-degree wind directions 

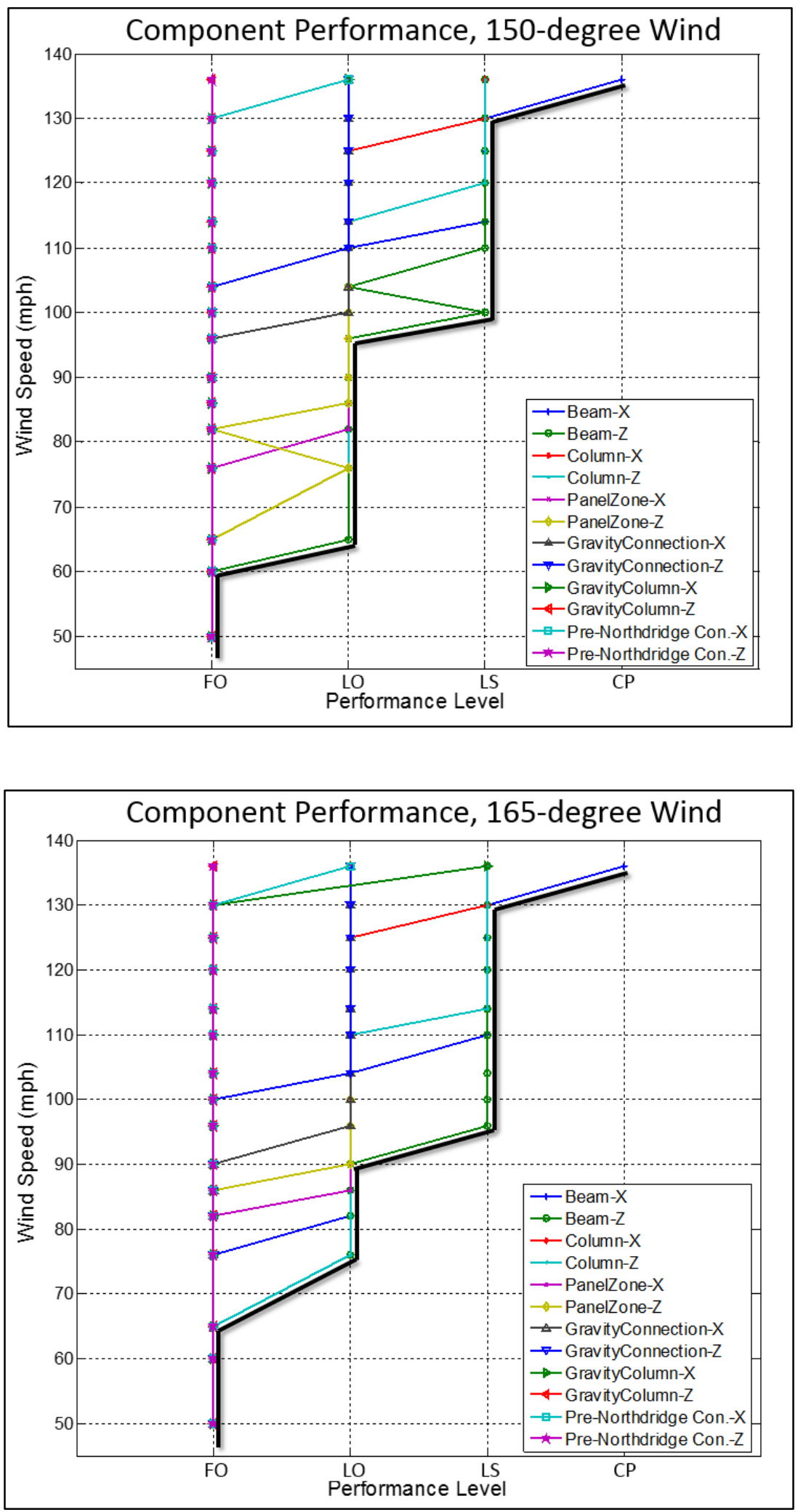

Figure 7-5. Component performance assessment of the 47-sotry building for 150 and 165-degree wind directions 

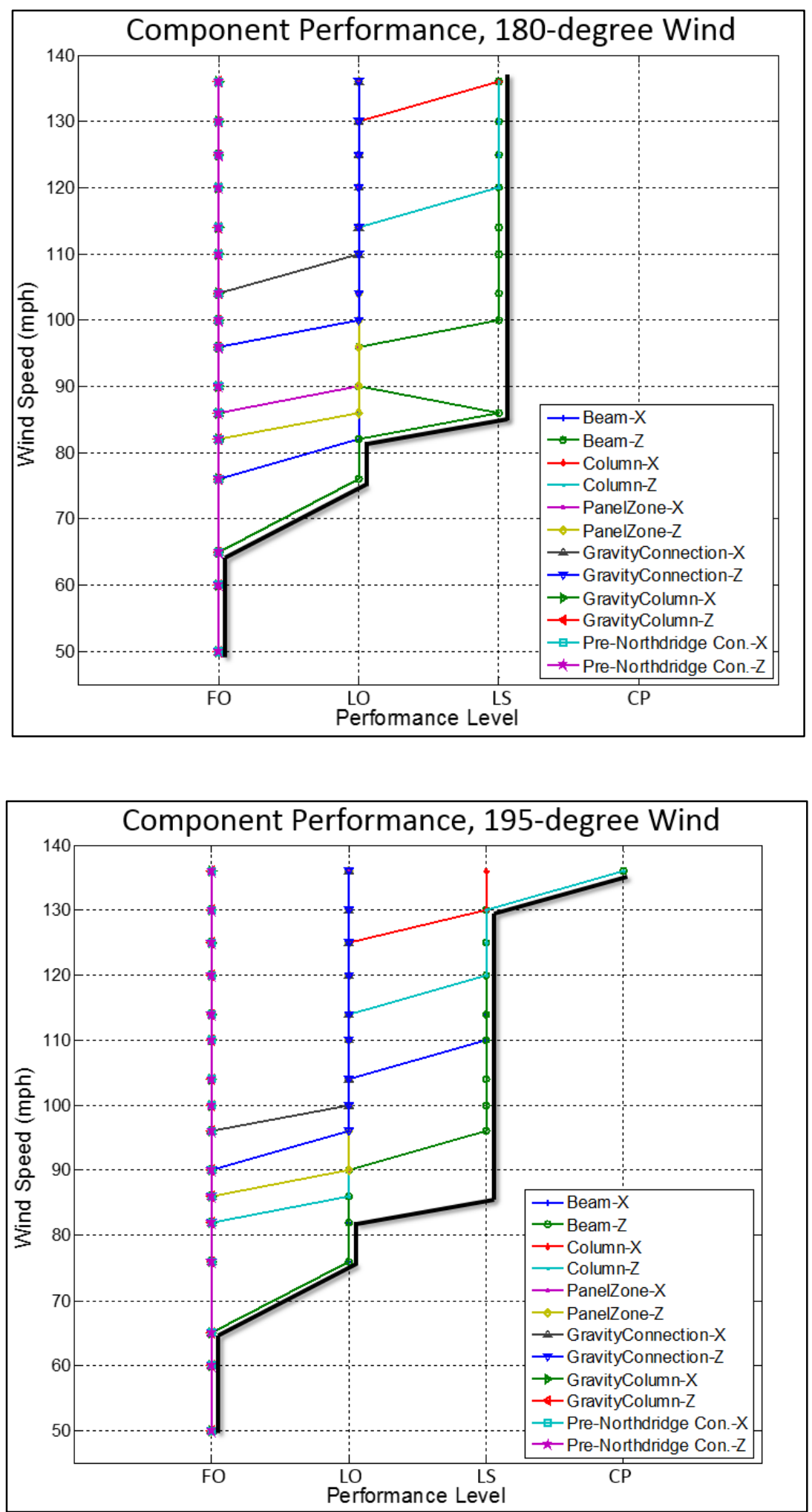

Figure 7-6. Component performance assessment of the 47-sotry building for 180 and 195-degree wind directions 
The envelope of the component performance status over the all critical wind directions was determined as the building's overall structural component performance. Figure 7-7 represents the overall component performance by including performance responses of the components in all six critical wind directions $\left(120^{\circ}, 135^{\circ}, 150^{\circ}, 165^{\circ}, 180^{\circ}\right.$ and $\left.195^{\circ}\right)$ obtained from IDA results. According to the general component performance envelope shown in Figure 7-7, it can be concluded that the performance status of the building's components will be as follows: (1) 0 to 60 mph: Fully Operational (FO); (2) 60 to $82 \mathrm{mph}$ : Limited Operations (LO); (3) 82 to $130 \mathrm{mph}$ : Life Safety (LS); and (4) 130 to $136 \mathrm{mph}$ : Collapse Prevention (CP).

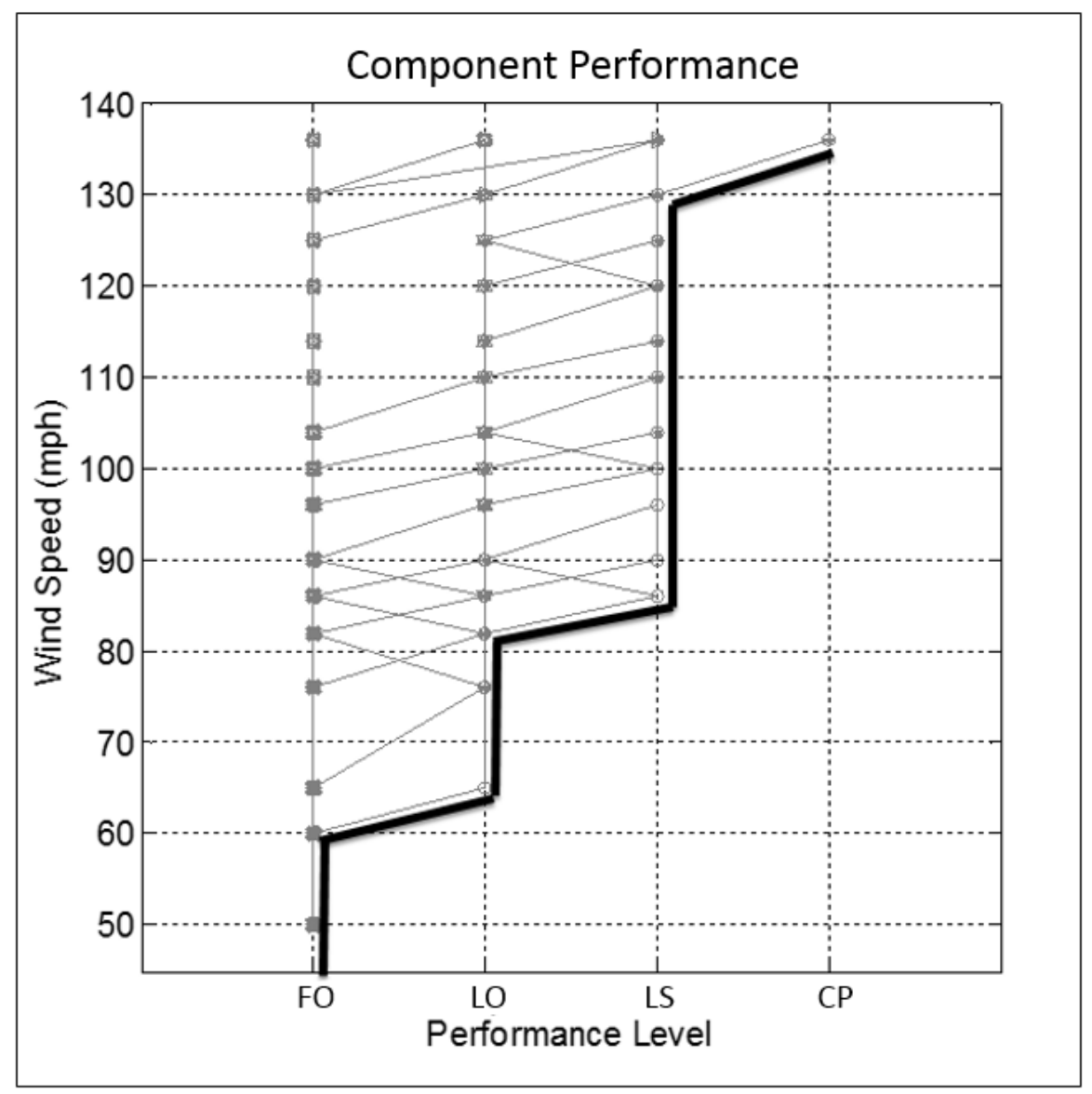

Figure 7-7. General component performance assessment of the 47-story building based on all six critical wind directions

According to available records, Hurricane Alicia struck the building with approximately maximum $82 \mathrm{mph}$ basic wind speed in the critical directions of the building. Survey and visual 
inspections conducted after the hurricane indicated no significant structural damage to the structure due to wind loads. According to the conducted assessment, for a wind speed of $82 \mathrm{mph}$, the LO performance level is expected which is associated with limited minor yielding in steel components. This estimation is in a good agreement with the reported observations.

\section{Cladding Performance to Wind-Induced Shear Deformation}

To assess the cladding performance two cladding panels, including exterior and interior panels, were selected as the Drift Damage Zones (DDZs) as shown in Figure 7-8. The peak DDI values for both DDZs in both major building axes were obtained from IDAs. It should be noted that the displacements related to building's corners in each floor were only available from the IDA results. Therefore, to estimate the DDI in specified DDZs, pushover analyses in both principle directions of the building using the first mode shape pattern were performed. Using the pushover results, for each floor the coefficients relating the DDIs of the specified DDZs to DDI of overall building width were calculated. Since the wind response of the building was dominated by the first mode response, the obtained coefficients were utilized to determine the DDIs for the DDZs from the DDI of overall building width in each story. The average responses over the six critical wind directions for both DDZs were compared to the performance levels as shown in Figure 7-9. As shown in the figure the interior cladding panels experienced relatively larger shear deformation DDI than the exterior cladding panels. It is shown that due to the flexibility of the building, relatively moderate wind speeds are associated with significant expected damage to the cladding system. According to the suggested performance guidelines proposed herein, for a 50year MRI (104 mph) defined as the FO level, a 100-year MRI (114 mph) defined as the LO level and a 700-year MRI (136 mph) defined as the LS level, are respectively required, but the actual predicted performances in this study for these wind speeds all exceeded the LS performance level and therefore were not acceptable. According to Figure 7-9, it can be concluded that the predicted 
performance level status of the building's exterior cladding will be as following: (1) 0 to $50 \mathrm{mph}$ : Fully Operational (FO); (2) 50 to $65 \mathrm{mph}$ : Limited Operations (LO); and (3) 65 to $74 \mathrm{mph}$ : Life Safety (LS). This assessment is in agreement with Building's history records revealing noises due to the interaction between the structure and the cladding for wind exceeding $20 \mathrm{mph}$. Records also indicate frequent elevator problems due to large building sway. While significant damage to cladding due to Hurricane Alicia was reported, it was difficult to differentiate the damage contribution caused by wind-borne debris and the large shear deformation.

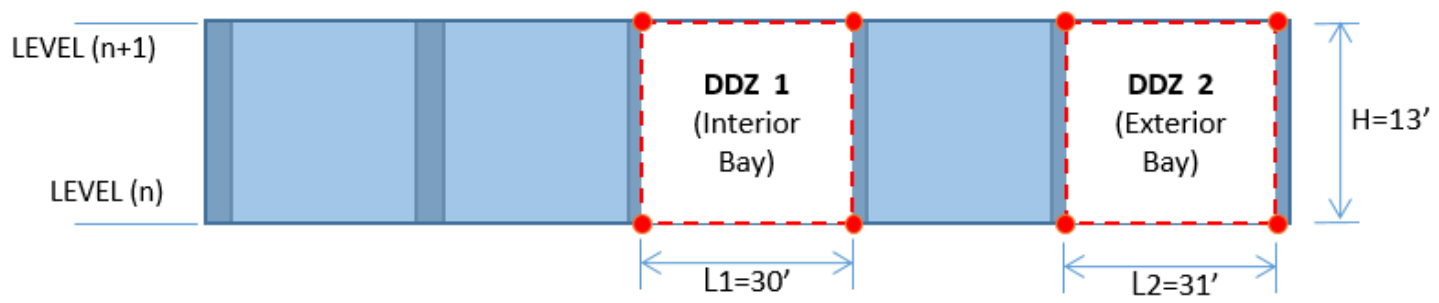

Figure 7-8. Interior and exterior drift damage zones (DDZs) in the 47-story building considered for cladding performance assessment 


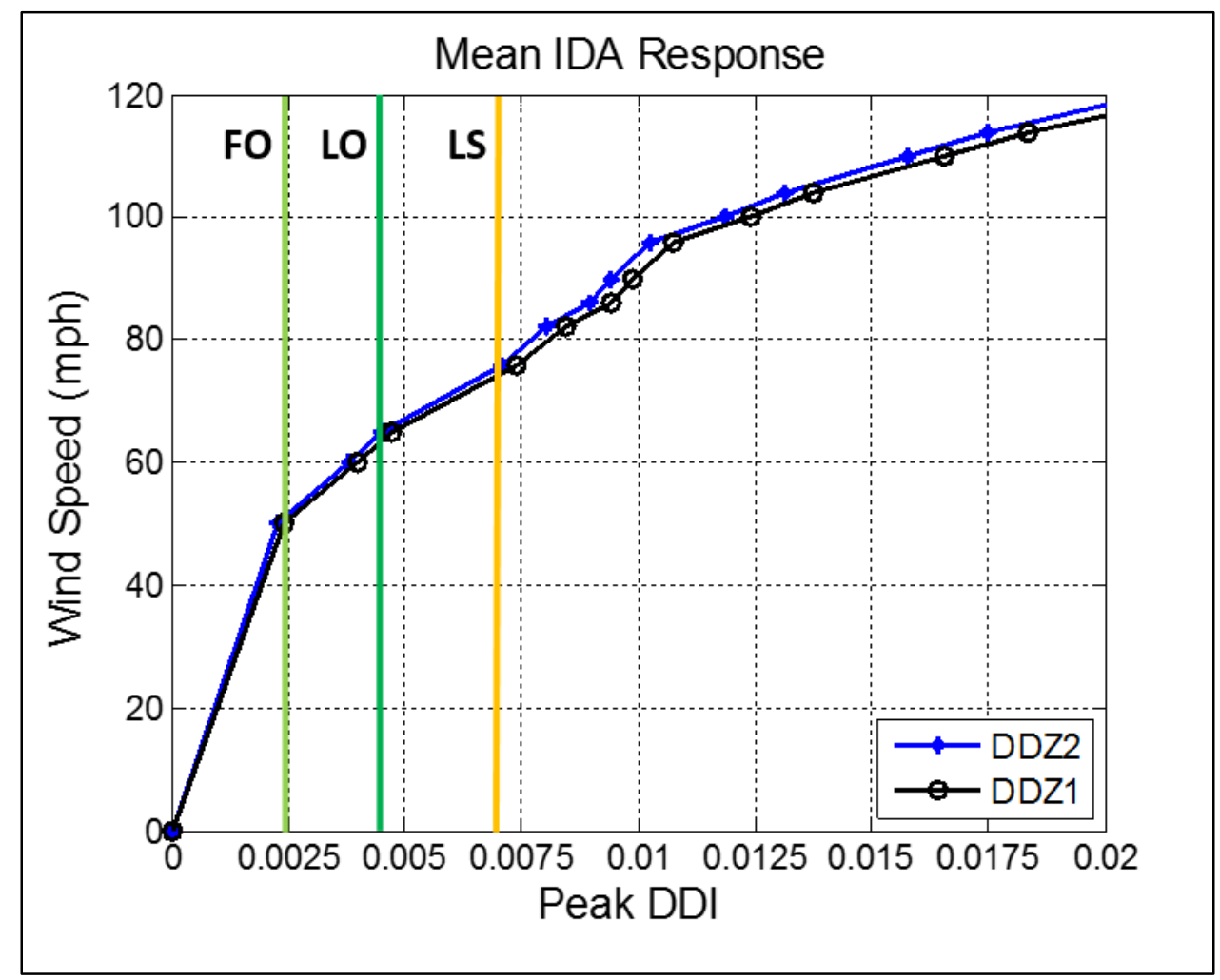

Figure 7-9. Assessment of the performance of cladding system of the 47-story building to windinduced shear deformation

\section{Serviceability Motion Comfort Performance}

Figure 7-10 represents the peak floor acceleration in the dominant response direction (Zdirection) for six critical wind directions obtained from IDA results. The average of the IDA in critical wind directions is shown with a thick black curve which is considered as the building's overall response. The performance levels and the corresponding acceptance criteria are also shown in the figure to enable the serviceability performance over the full range of the basic wind speeds to be estimated. The motion comfort performance is normally evaluated at a wind hazard level corresponding to the 1- to 10-year MRI. For the subject building's location, the 10-year MRI corresponds to a $76 \mathrm{mph}$ basic wind speed. The figure shows that $76 \mathrm{mph}$ for thus building relates to a very annoying performance, which is not acceptable. The overall estimated motion comfort performance status of the building will be as follows: (1) 0 to $24 \mathrm{mph}$ basic wind speed: 
Not Perceptible motion less than $5 \mathrm{mg}$; (2) 24 to $56 \mathrm{mph}$ basic wind speed: Threshold of Perceptibility at 5-15 mg; (3) 60 to $64 \mathrm{mph}$ basic wind sped: Target Level for office buildings at $20-25 \mathrm{mg} ; 67-74 \mathrm{mph}$ basic wind speed: Annoying motion at 28 to $40 \mathrm{mg}$; (4) 74 to $116 \mathrm{mph}$ basic wind speed: Very Annoying motion at 40 - $150 \mathrm{mg}$; and (5) > $116 \mathrm{mph}$ basic wind speed: Intolerable motion above $150 \mathrm{mg}$. Consistent with the results obtained in this study, building inspection reports after Hurricane Alicia indicated movement of chairs across floors and displaced ceiling tiles in the subject building discussed here and difficulty in walking due to the severe lateral sway and measured peak accelerations of $43 \mathrm{mg}$ in a nearby steel building (Isyumov \& Halvorson, 1984) .

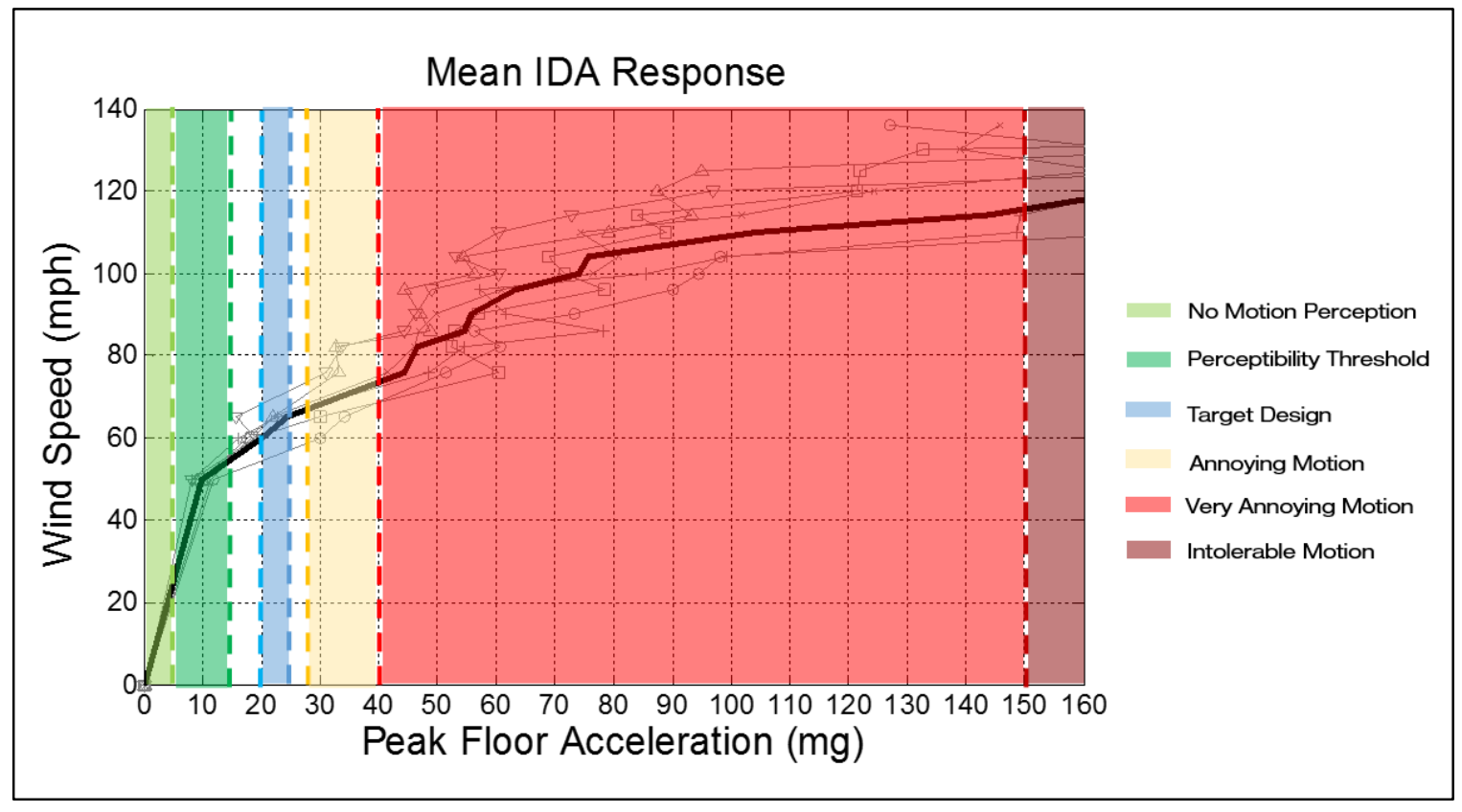

Figure 7-10. Assessment of the serviceability performance of the 47-story building related to motion comfort

ISO 10137 (ISO, 2004) suggests that serviceability design of buildings for wind-induced vibrations be based on 1-year MRI wind, in accordance with two frequency-dependent acceptance curves for residential and office buildings as shown in Figure 7-11. For the 47-story office building, the estimated peak wind-induced accelerations in both building's principle 
directions based on a $62 \mathrm{mph}$ basic wind speed (1-year MRI) are shown in Figure 7-11 with respect to ISO acceptance limits. It is shown that the estimated peak accelerations for this building are higher than the acceptance limit curve for office buildings.

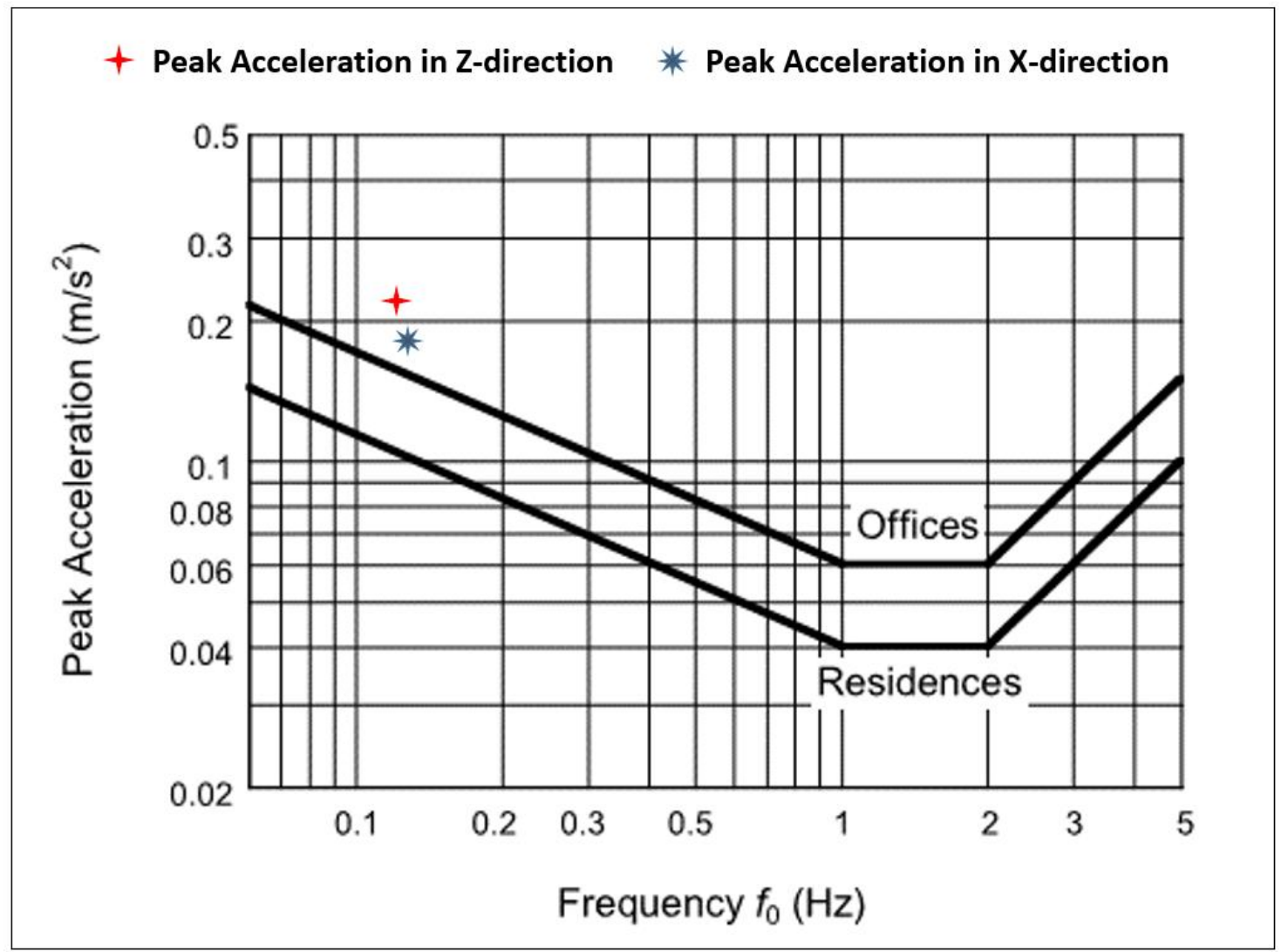

Figure 7-11.10-year MRI peak acceleration responses of the 47-story building in $X$ and Z directions in respect to basic evaluation curves for wind-induced vibration

Generally, the cladding and serviceability performance assessment indicates that the building cannot provide acceptable performance regarding the inter-story drift and floor acceleration and extra stiffening was required to reach the performance objectives according to the suggested framework. These issues were addressed in later strengthening and renovation. 


\subsubsection{Wind Performance Assessment of The 30-Story Building}

\section{Structural Component Performance}

For the 30-story building, the wind performance assessment of the structural components was conducted by evaluating the responses of all existing components resulting from IDAs with respect to the acceptance criteria. Figure 7-12 and Figure 7-13 represent the component performance evaluations for $0^{\circ}, 15^{\circ}, 30^{\circ}$ and $45^{\circ}$ wind directions. In the figures, the thick black lines represent the estimated performance of the entire building by enveloping the performances of all the components. For example, for $15^{\circ}$ wind direction, it is shown that at $70 \mathrm{mph}$ wind speed the component performance changes from Fully Operational (FO) to Limited Operations (LO). Maintaining the LO performance level from $76 \mathrm{mph}$ to $150 \mathrm{mph}$, the component performance level changes to Life Safety (LS) level at $155 \mathrm{mph}$ basic wind speed. It remains at the same performance level until the building collapse level corresponding to $170 \mathrm{mph}$ where it finally reaches the Collapse Prevention $(\mathrm{CP})$ performance level. Reviewing the estimated performance for the wind direction range, indicates a trend of lower responses by changing the wind direction from $0^{\circ}$ to $45^{\circ}$. 

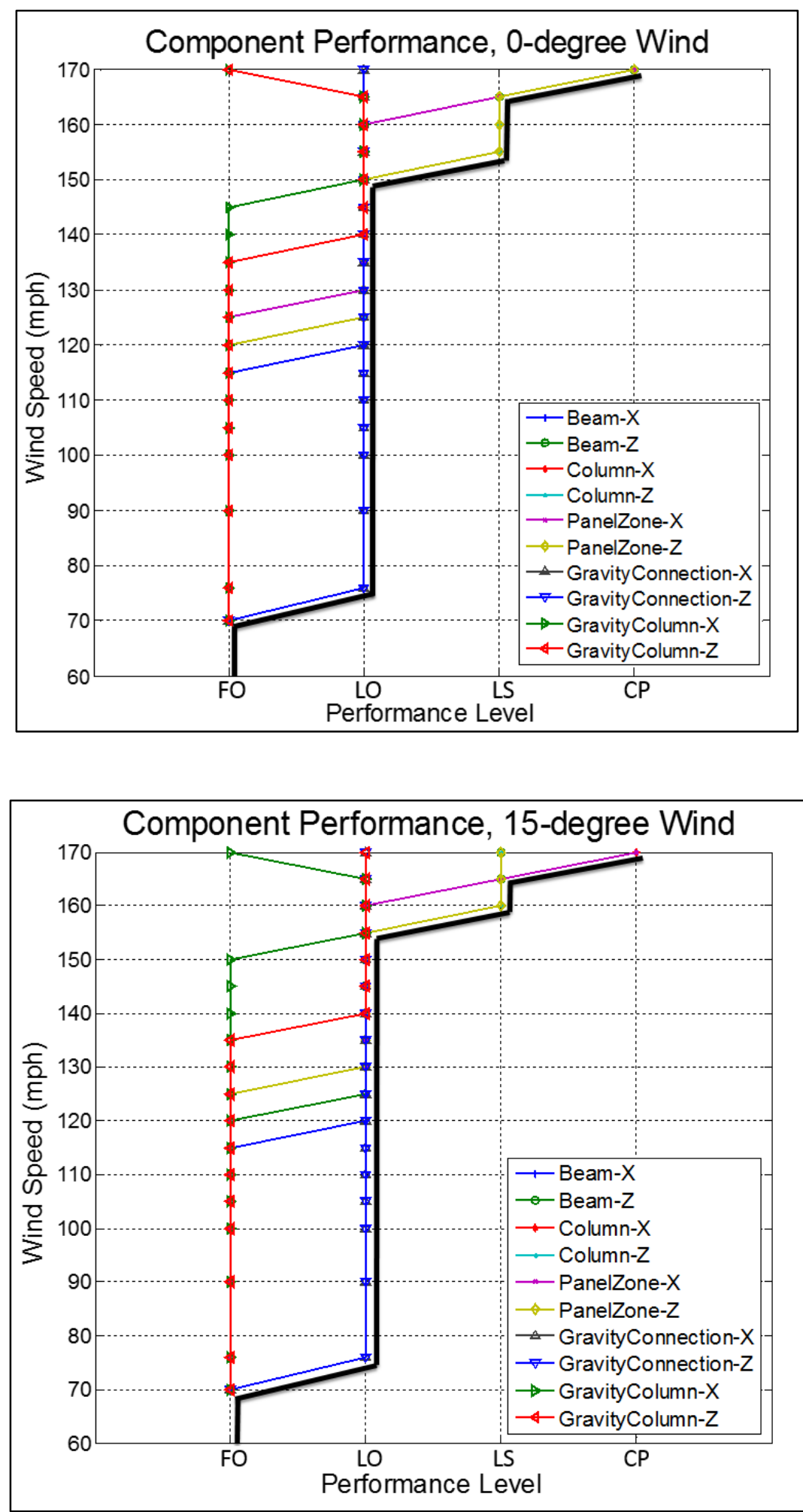

Figure 7-12. Component performance assessment of the 30-story building for zero and 15-degree wind directions 

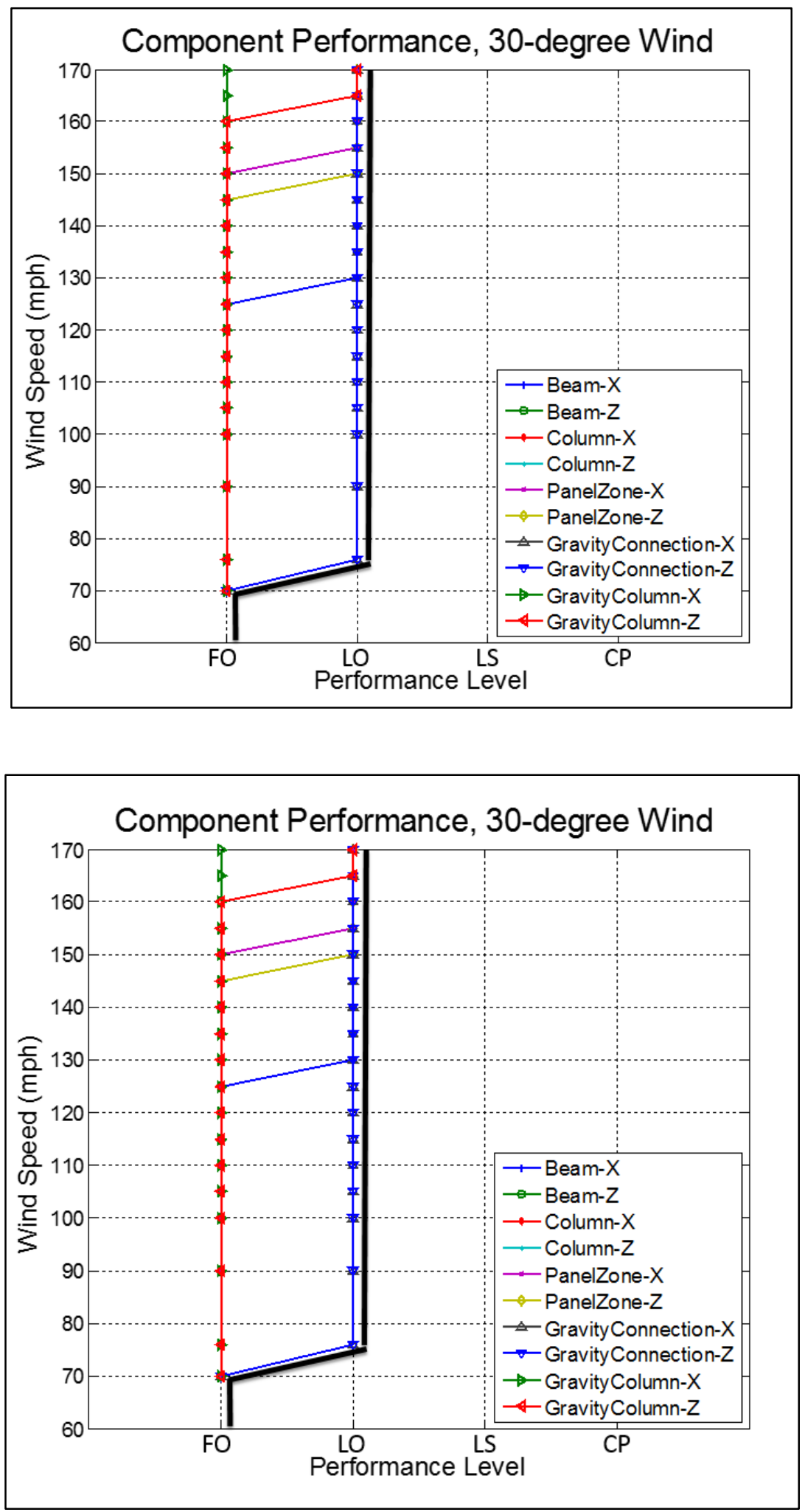

Figure 7-13. Component performance assessment of the 30-story building for 30 and 45-degree wind directions 
Figure 7-14 shows the overall component performance by enveloping the performance responses of the structural components in all four wind direction from $0^{\circ}$ to $45^{\circ}$. According to the general component performance envelope, it can be concluded that the performance status of the building's structural components will be as following: (1) 0 to 70mph Fully Operational (FO); (2) 70 to $150 \mathrm{mph}$ : Limited Operations (LO); (3) 150 to 165mph: Life Safety (LS) and finally 165 to 170mph: Collapse Prevention (CP). The framework suggested by Griffis et al. requires the building to meet: FO for 50-year MIR (90mph); LO for 100-year MIR (96mph); and LS for 700year MIR (115mph). The current wind performance estimation suggests that building fails to provide FO for $90 \mathrm{mph}$ but it meets the two other performance objectives.

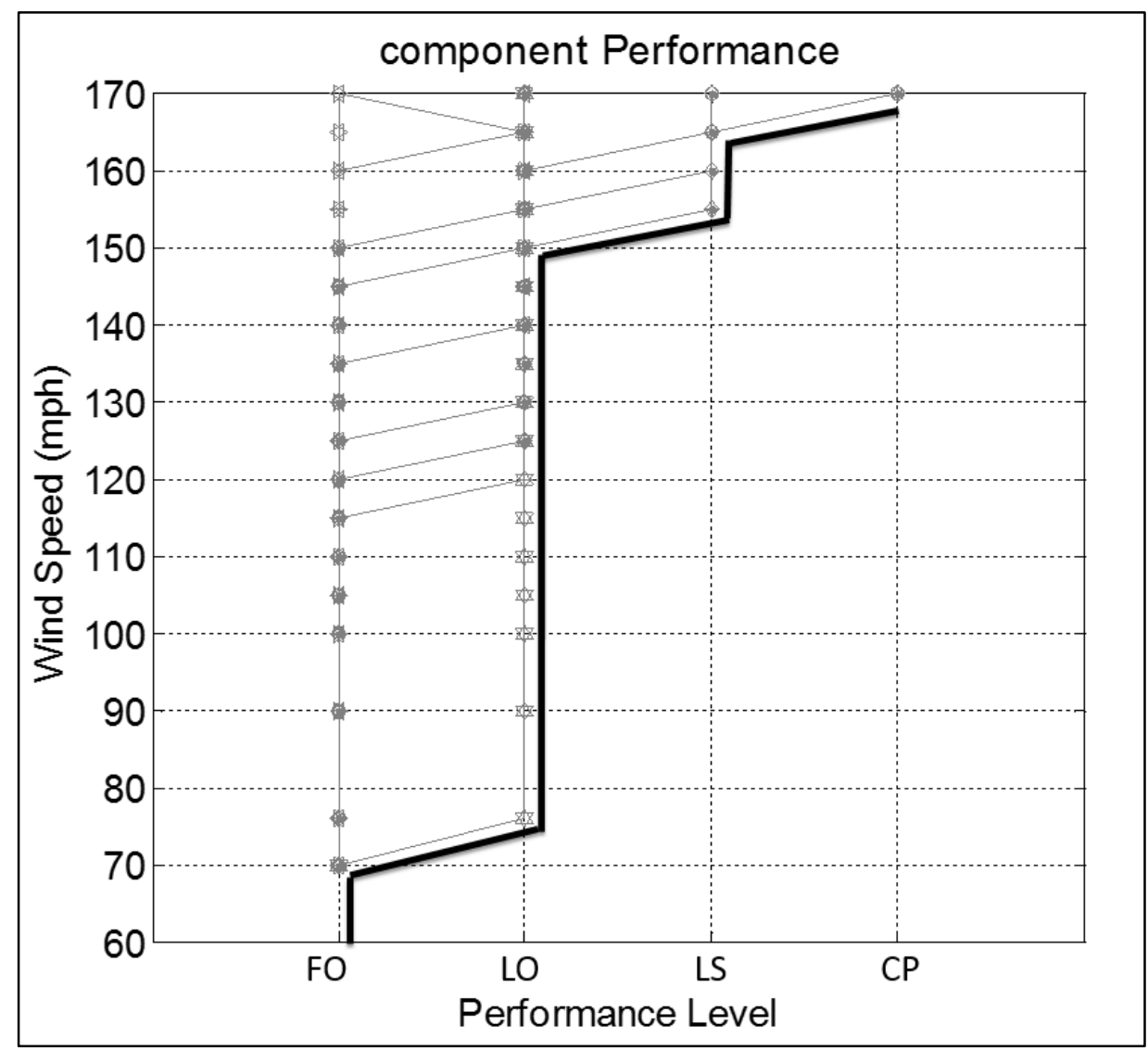

Figure 7-14. General component performance assessment of the 30-story building based on all four wind directions 


\section{Cladding Performance to Wind-Induced Shear Deformation}

For the 30-story building, cladding performance under wind-induced shear deformation was evaluated for two cladding panels including an exterior and an interior cladding panel as the Drift Damage Zones (DDZs), shown in Figure 7-15. The peak DDI values for both DDZs in both major building axes were obtained from IDAs along with coefficients measured from pushover analyses relating the DDIs of the specified DDZs to the DDI of the overall building (the same procedure as explained for the 47-story building). Figure 7-16 shows the peak DDI averaged over the four critical wind directions for both DDZs compared to the performance levels. According to the figure, it can be concluded that the predicted performance level status of the building's cladding will be as following: (1) 0 to $51 \mathrm{mph}$ : Fully Operational (FO); (2) 51 to $80 \mathrm{mph}$ : Limited Operations (LO); and (3) 80 to $91 \mathrm{mph}$ : Life Safety (LS). According to the suggested performance guidelines, for a 50-year MRI (90 mph) defined as the FO level, a 100-year MRI (96 mph) defined as the LO level and a 700-year MRI (115 mph) defined as the LS level, are respectively required. According to estimated performance, for cladding performance to windinduced shear deformation generally, the 30-story building fails to meet the performance objectives.

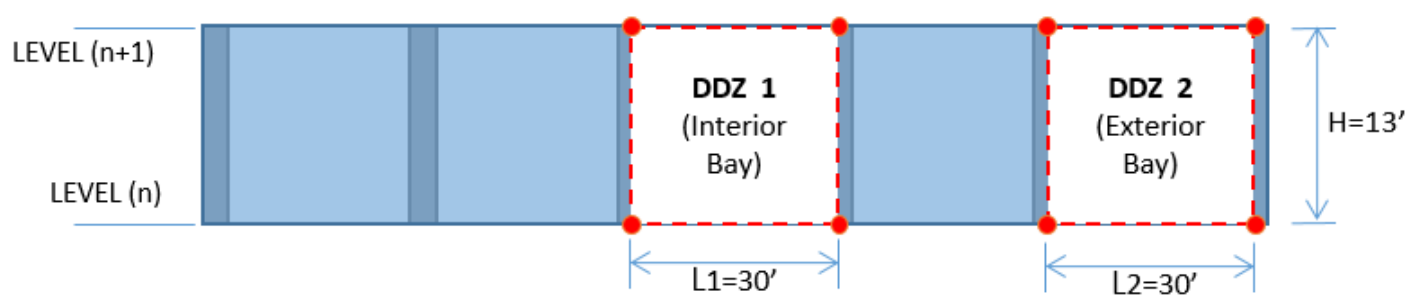

Figure 7-15. Interior and exterior drift damage zones (DDZs) in the 30-story building considered for cladding performance assessment 


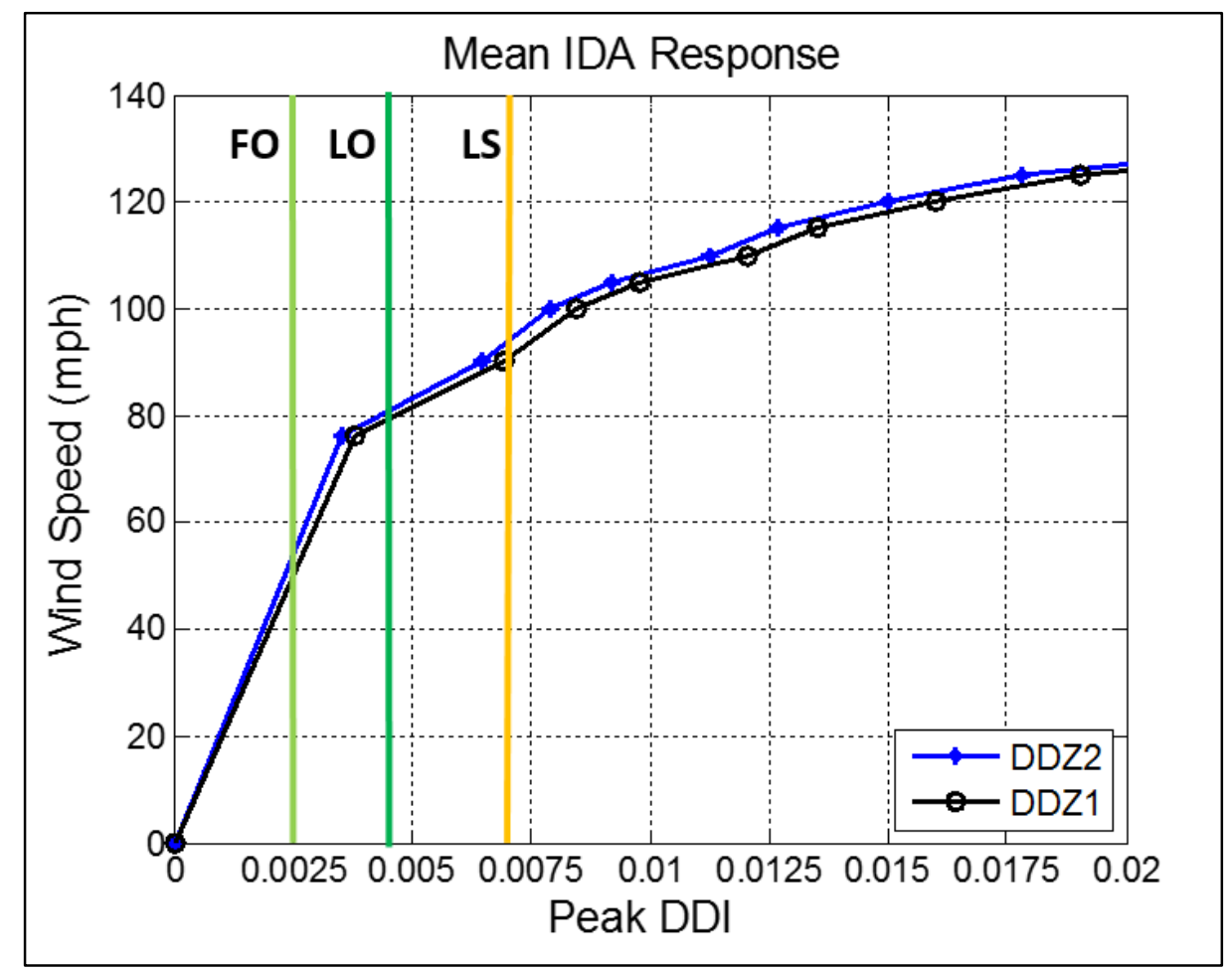

Figure 7-16. Assessment of performance of cladding system of the 30-story building to windinduced shear deformation

\section{Serviceability Motion Comfort Performance}

The peak floor acceleration for four critical wind directions obtained from IDA results in the dominant response direction (Z-direction) is shown in Figure 7-17. The building's overall response is obtained from the averaging the peak IDA results over the critical directions shown with a thick black curve in the figure. The performance levels and the corresponding acceptance criteria were also shown in the figure to enable the estimation of the serviceability performance over the full range of the basic wind speeds. The overall estimated motion comfort performance status of the building is as follows: (1) 0 to $22 \mathrm{mph}$ basic wind speed: Not Perceptible motion less than $5 \mathrm{mg}$; (2) 22 to $68 \mathrm{mph}$ basic wind speed: Threshold of Perceptibility at $5-15 \mathrm{mg}$; (3) 79 to $84 \mathrm{mph}$ basic wind speed: Target Level for office buildings at $20-25 \mathrm{mg} ; 87-96 \mathrm{mph}$ basic wind speed: Annoying motion at 28 to $40 \mathrm{mg}$; (4) 96 to $138 \mathrm{mph}$ basic wind speed: Very Annoying motion at 40 - $150 \mathrm{mg}$; and (5) > $138 \mathrm{mph}$ basic wind speed: Intolerable motion above 
$150 \mathrm{mg}$. For the subject building with the 10-year MRI wind equal to $76 \mathrm{mph}$ basic wind speed, the response curve corresponds to $17 \mathrm{mg}$ peak acceleration acceptable for an office building. Figure 7-18 shows the estimated peak wind-induced accelerations in both of the building's principal directions based on a $62 \mathrm{mph}$ basic wind speed (1-year MRI) along with the ISO frequency-dependent acceptance curves. It is shown that the peak acceleration in the Z-direction slightly exceeded the office building limit. However, the peak acceleration in X-direction is in the acceptable range.

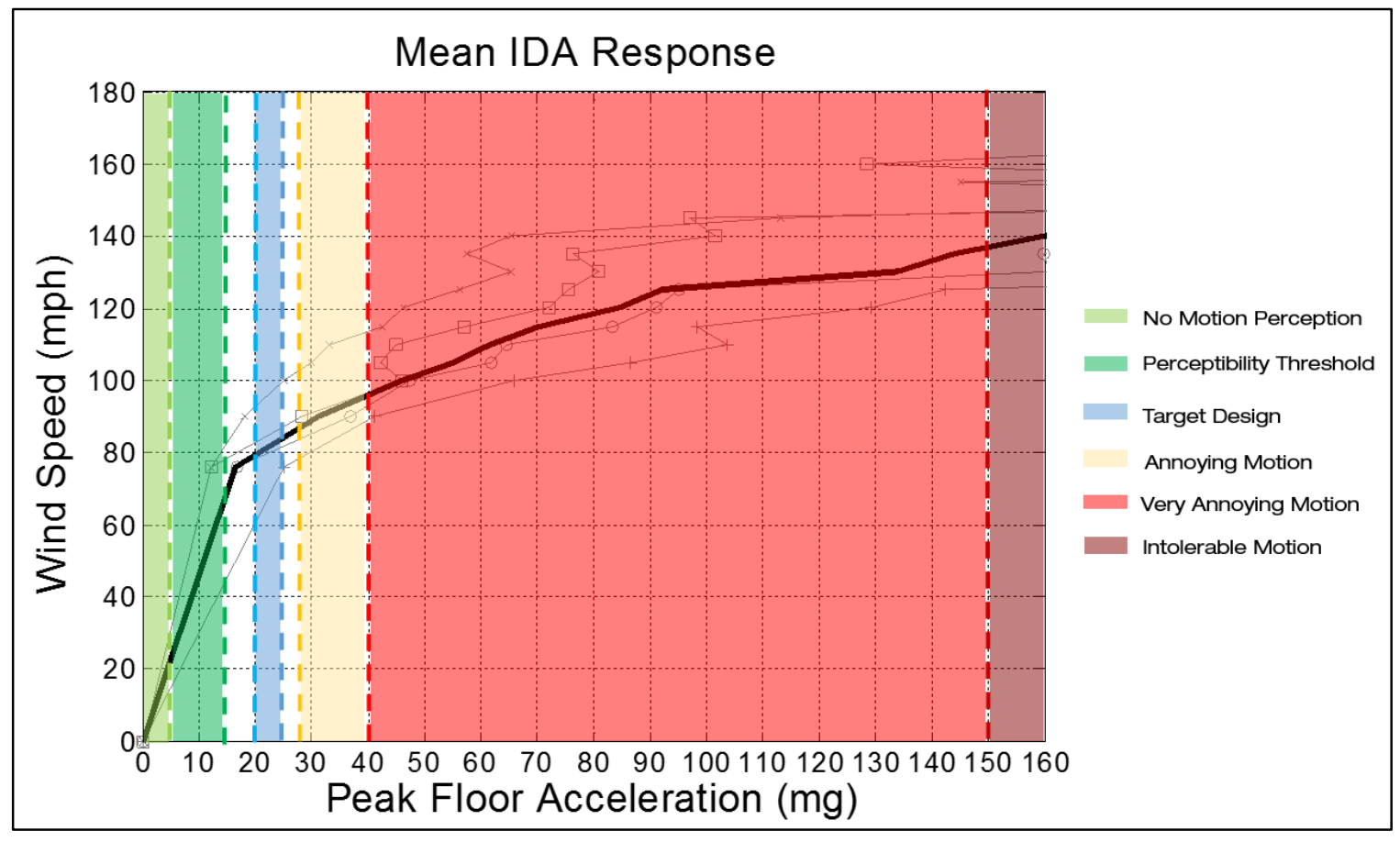

Figure 7-17. Assessment of the serviceability performance of the 30-story building related to motion comfort 


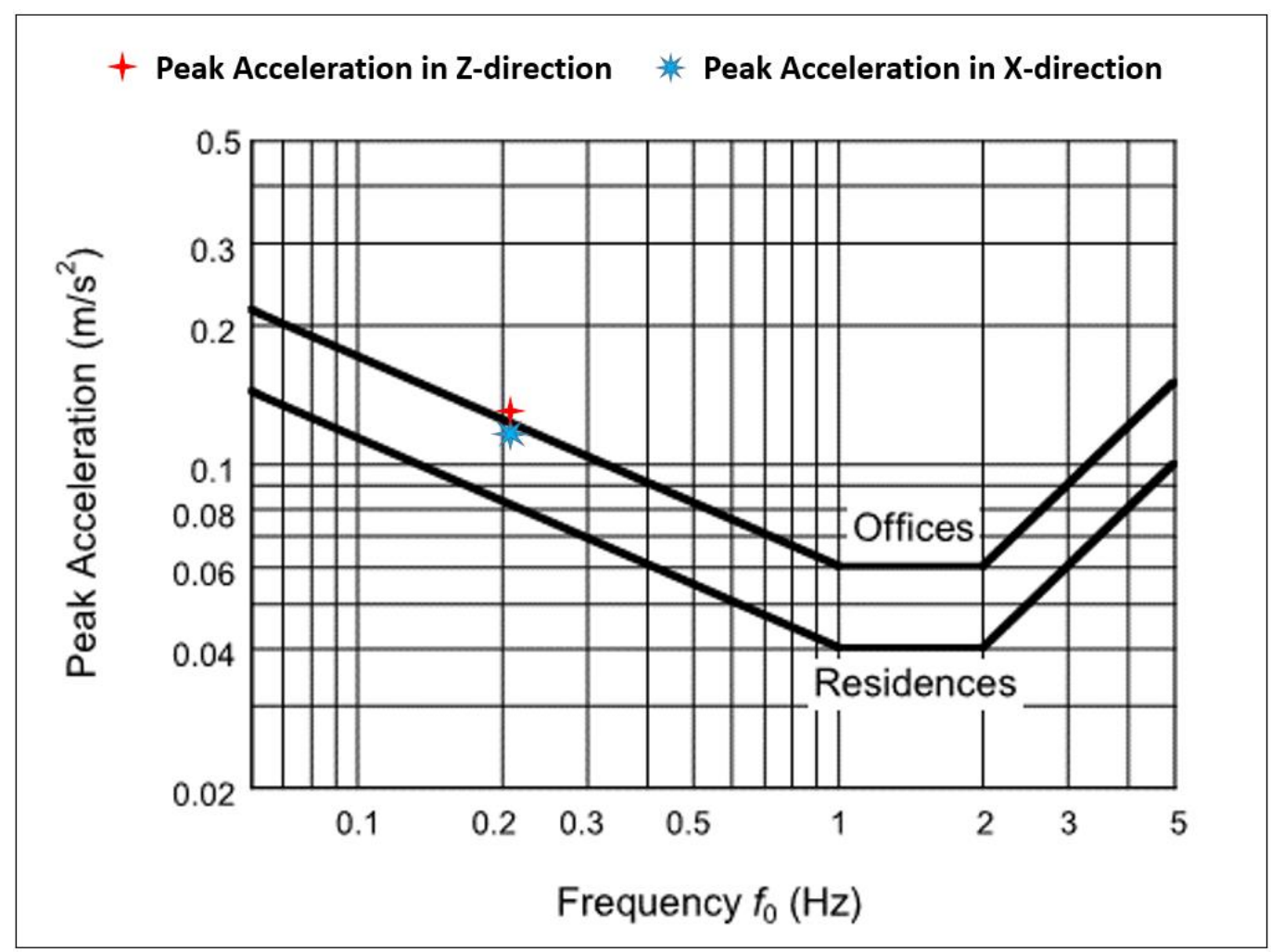

Figure 7-18. 10-year MRI peak acceleration responses of the 30-story building in $X$ and $Z$ directions in respect to basic evaluation curves for wind-induced vibration

\subsubsection{Wind Performance Assessment of The 40-Story Building}

\section{Structural Component Performance}

Figure 7-19 and Figure 7-20 show the wind performance assessment of the structural components for the 40-story building conducted by evaluating the responses of all existing components resulting from IDA. In the figures, the thick black lines represent the estimated performance of the entire building by enveloping the performances of all the components for $0^{\circ}, 15^{\circ}, 30^{\circ}$ and $45^{\circ}$ wind directions. For example, for $15^{\circ}$ wind direction, it is shown that at $70 \mathrm{mph}$ wind speed the component performance changes from Fully Operational (FO) to Limited Operations (LO). Maintaining the LO performance level from $76 \mathrm{mph}$ to $125 \mathrm{mph}$, the component performance level changes to Life Safety (LS) level at $130 \mathrm{mph}$ basic wind speed. It remains at the same performance level until the building collapse level. From the figure, the same trend of lower 
responses by changing the wind direction from $0^{\circ}$ to $45^{\circ}$ is also shown as was concluded for the 30-story building performance assessment.
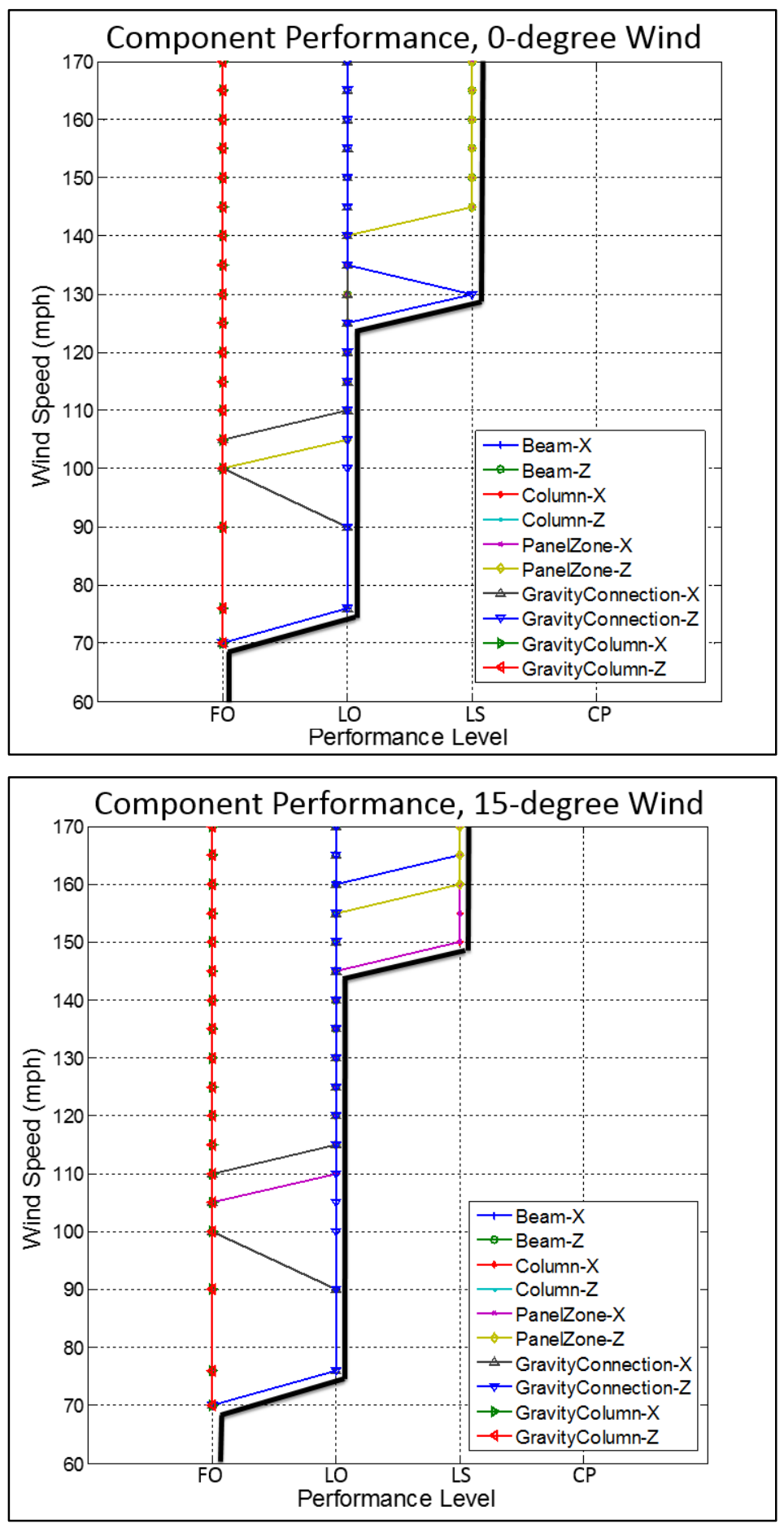

Figure 7-19. Component performance assessment of the 40-story building for zero and 15-degree wind directions 

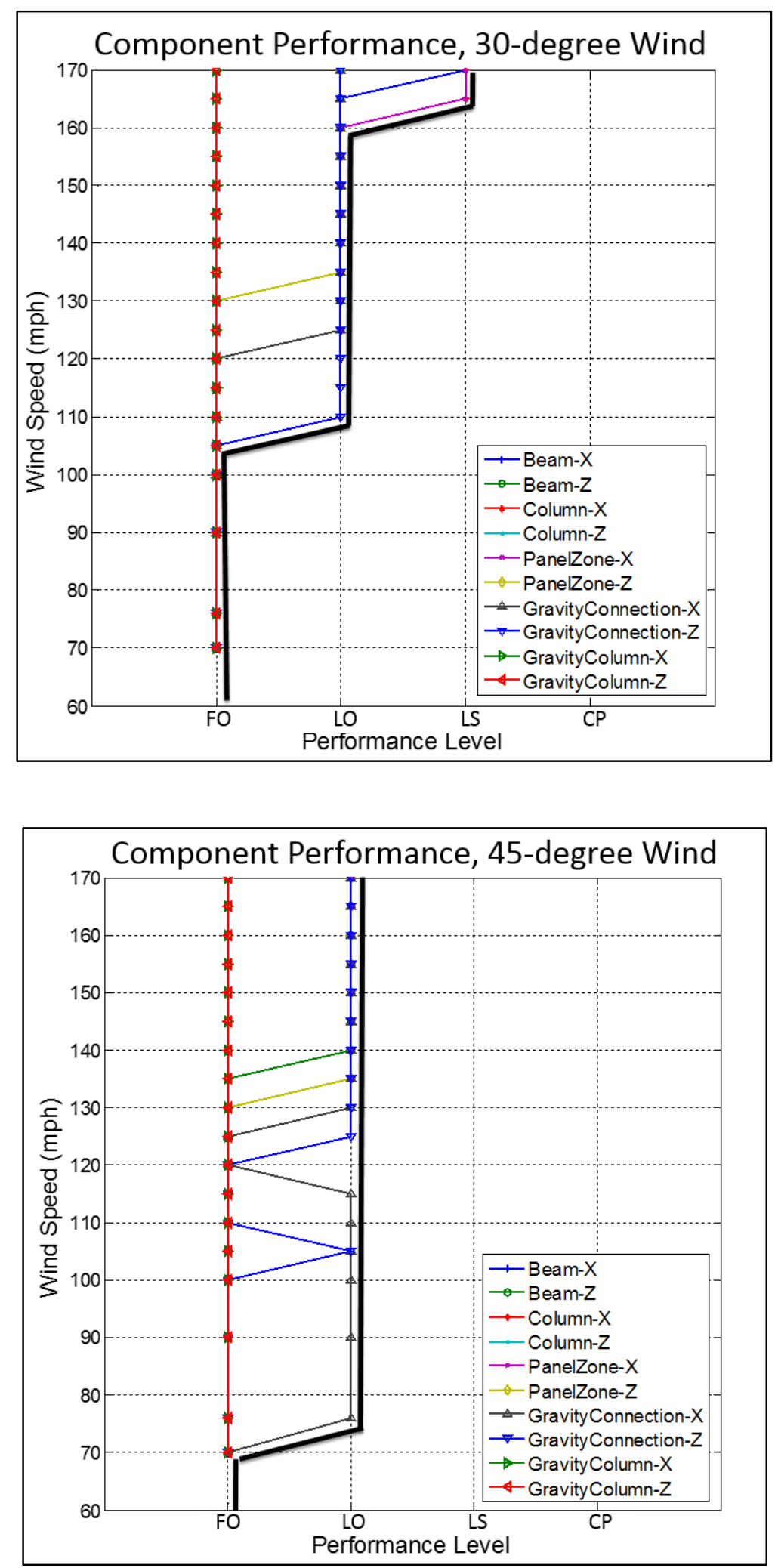

Figure 7-20. Component performance assessment of the 40-story building for 30 and 45-degree wind directions 
Figure 7-21 shows the overall component performance by including performance responses of the components in all four wind direction from $0^{\circ}$ to $45^{\circ}$ obtained from IDA results. According to general component performance envelope shown in Figure 7-21, it can be concluded that the performance status of the building's components will be as following: (1) 0 to 70mph Fully Operational (FO); (2) 70 to $125 \mathrm{mph}$ : Limited Operations (LO); (3) 125 to 170mph: Life Safety (LS). While the framework requires: CO for 50-year MIR (90mph); Op for 100-year MIR (96mph); and LI for 700-year MIR (115mph). The current wind performance estimation suggests that building fails to provide FO for 90mph but it meets the two other performance objectives.

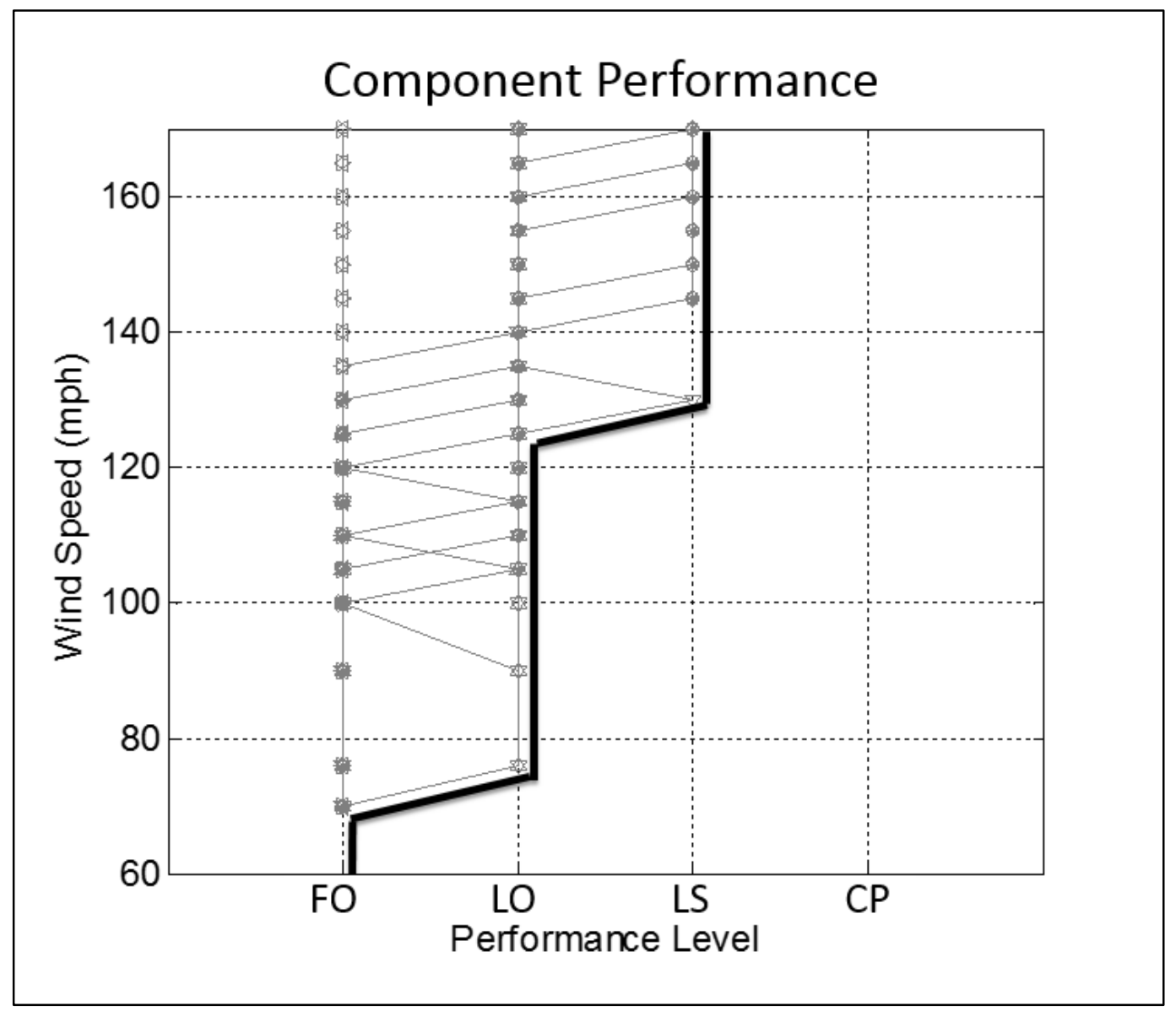

Figure 7-21. General component performance assessment of the 40-story building based on all four wind directions 


\section{Cladding Performance to Wind-Induced Shear Deformation}

The performance of the Cladding of the 40-story building under wind-induced shear deformation was evaluated for two Drift Damage Zones (DDZs) related to an exterior and an interior cladding panel as shown in Figure 7-22. Using the same procedure explained earlier the peak DDI values for both DDZs in both major building axes were obtained from IDAs along with coefficients measured from pushover analyses relating the DDIs of the specified DDZs to the DDI of the overall building. The peak DDI averaged over the four critical wind directions for both DDZs as the representative of overall cladding response, were compared to the performance levels as shown in Figure 7-23. According to the figure, the predicted performance level status of the building's cladding will be as following: (1) 0 to $72 \mathrm{mph}$ : Fully Operational (FO); (2) 72 to 93 mph: Limited Operations (LO); and (3) 93 to $106 \mathrm{mph}$ : Life Safety (LS). According to the suggested performance guidelines, for a 50-year MRI (90 mph) defined as the FO level, a 100year MRI (96 mph) defined as the LO level and a 700-year MRI (115 mph) defined as the LS level, are respectively required. According to the estimated performance, for cladding performance generally, the 40 -story building fails to meet the performance objectives. But it resulted in better cladding performance than the 30 -story building.

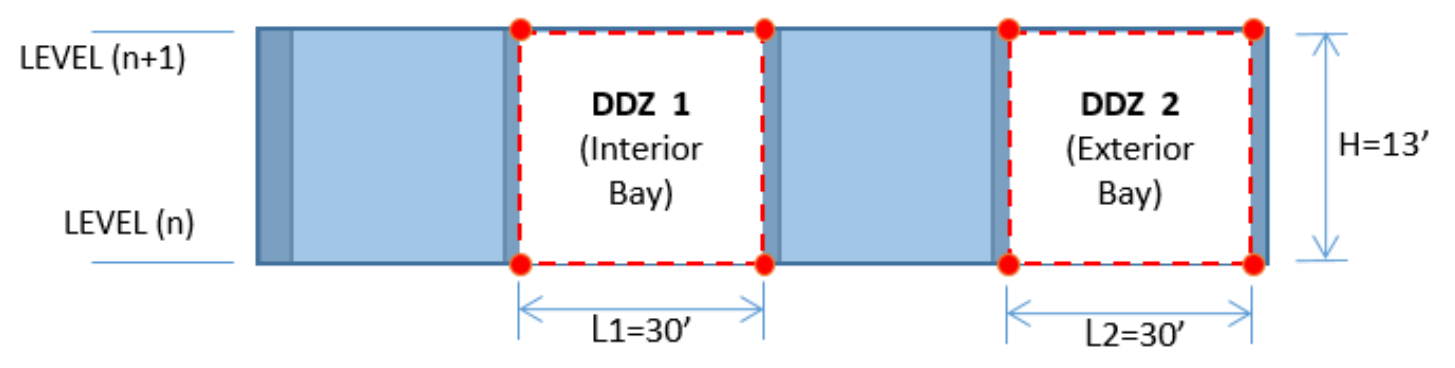

Figure 7-22. Interior and exterior drift damage zones (DDZs) in the 40-story building considered for cladding performance assessment 


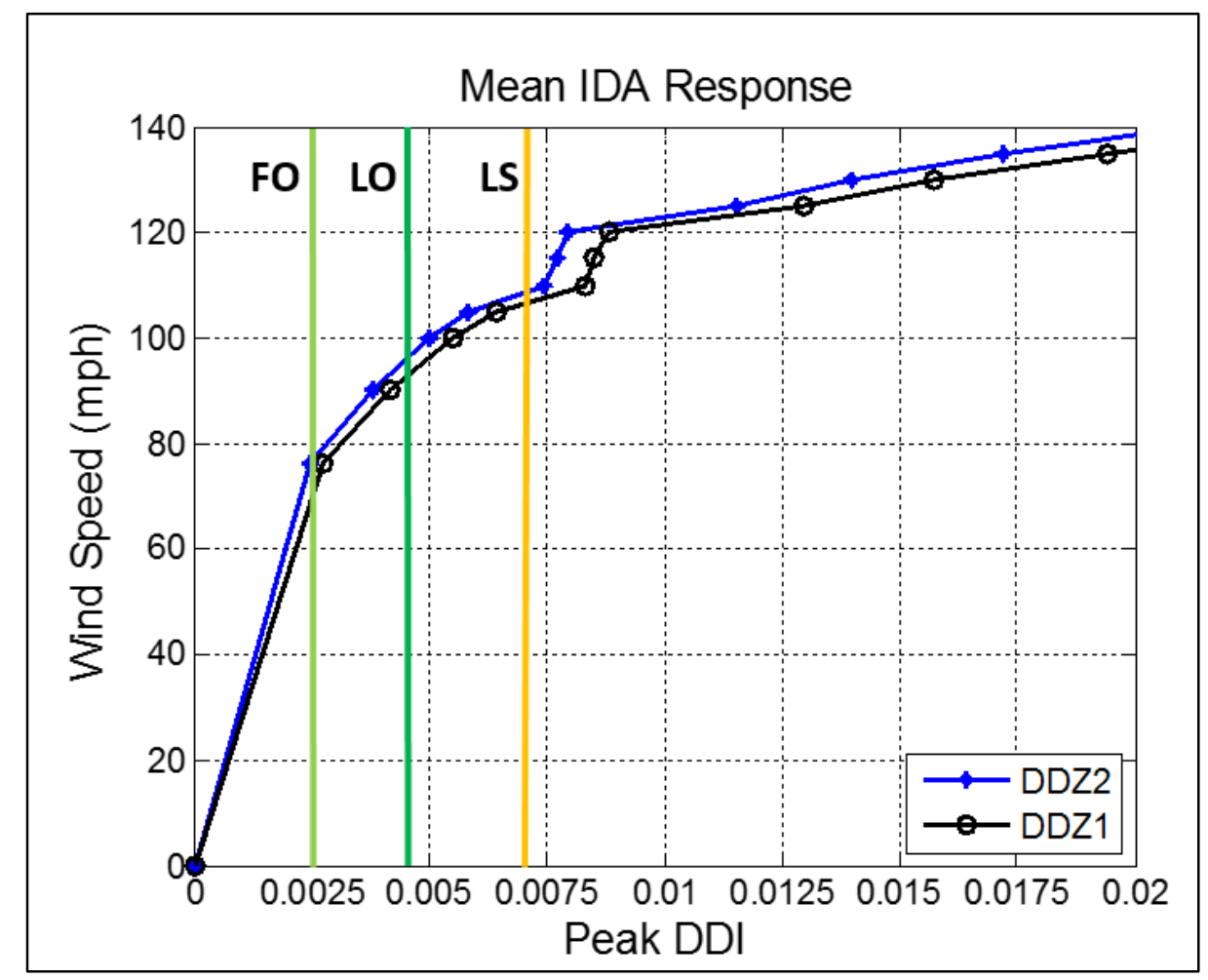

Figure 7-23. Assessment of performance of cladding system of the 40-story building to windinduced shear deformation

\section{Serviceability Motion Comfort Performance}

Figure 7-24 shows the building's overall peak acceleration curve along with the performance levels and the corresponding acceptance criteria representing the serviceability performance estimated over the full range of the basic wind speeds. The overall motion comfort performance status of the building is estimated as follows: (1) 0 to $15 \mathrm{mph}$ basic wind speed: Not Perceptible motion less than $5 \mathrm{mg}$; (2) 15 to $45 \mathrm{mph}$ basic wind speed: Threshold of Perceptibility at $5-15$ mg; (3) 62 to $76 \mathrm{mph}$ basic wind sped: Target Level for office buildings at $20-25 \mathrm{mg} ; 79-90$ mph basic wind speed: Annoying motion at 28 to $40 \mathrm{mg}$; (4) 90 to $127 \mathrm{mph}$ basic wind speed: Very Annoying motion at $40-150 \mathrm{mg}$; and (5) > $127 \mathrm{mph}$ basic wind speed: Intolerable motion above $150 \mathrm{mg}$. For the subject building with the 10 -year MRI wind equal to $76 \mathrm{mph}$ basic wind speed, the response curve corresponds to $25 \mathrm{mg}$ peak acceleration acceptable for an office building the estimated peak wind-induced accelerations in both of the building's principal 
directions based on a $62 \mathrm{mph}$ basic wind speed (1-year MRI) along with the ISO frequencydependent acceptance curves are shown in Figure 7-24. As shown in the figure, the peak acceleration in both directions significantly exceeds the ISO limit curve for office buildings.

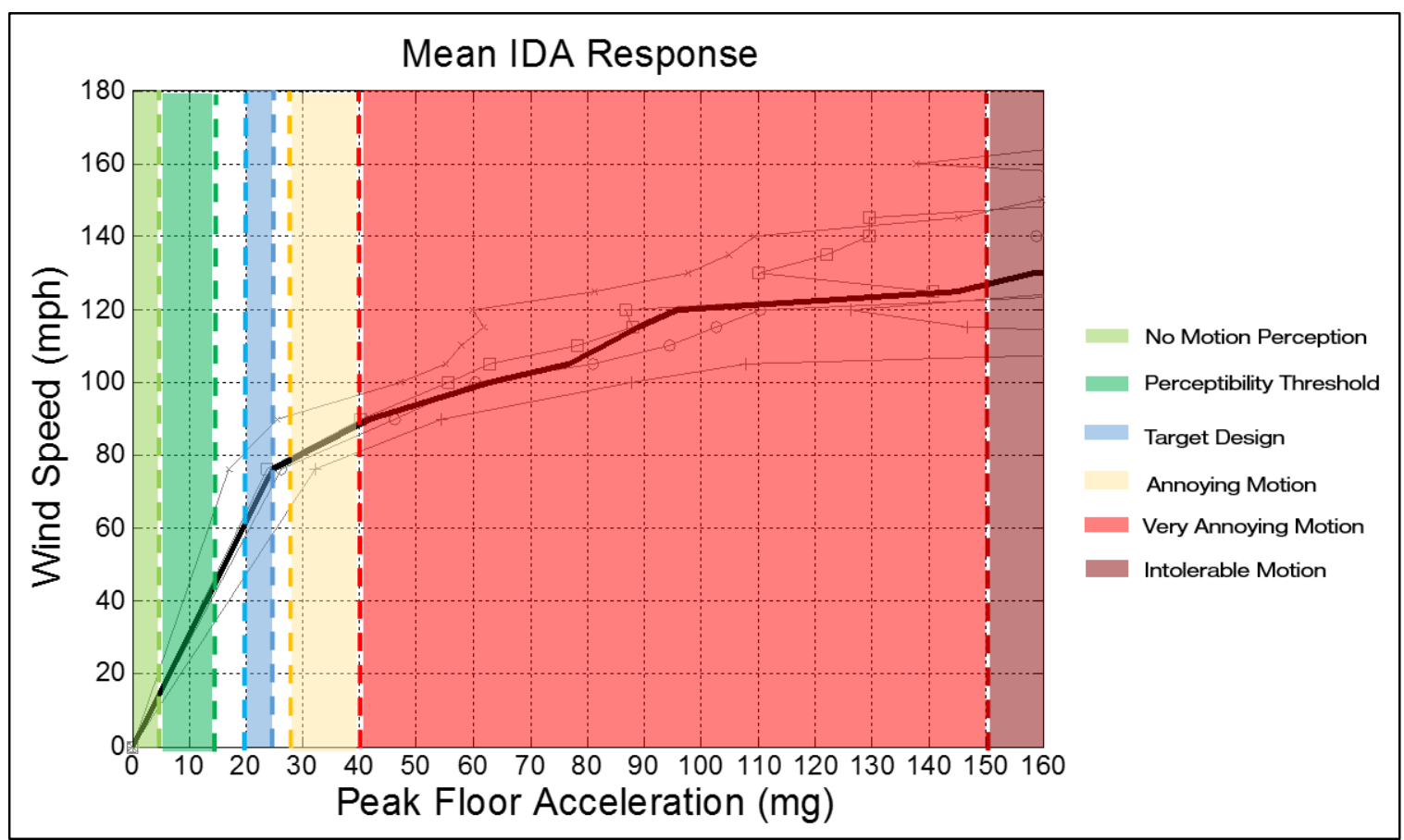

Figure 7-24. Assessment of the serviceability performance of the 40-story building related to motion comfort 


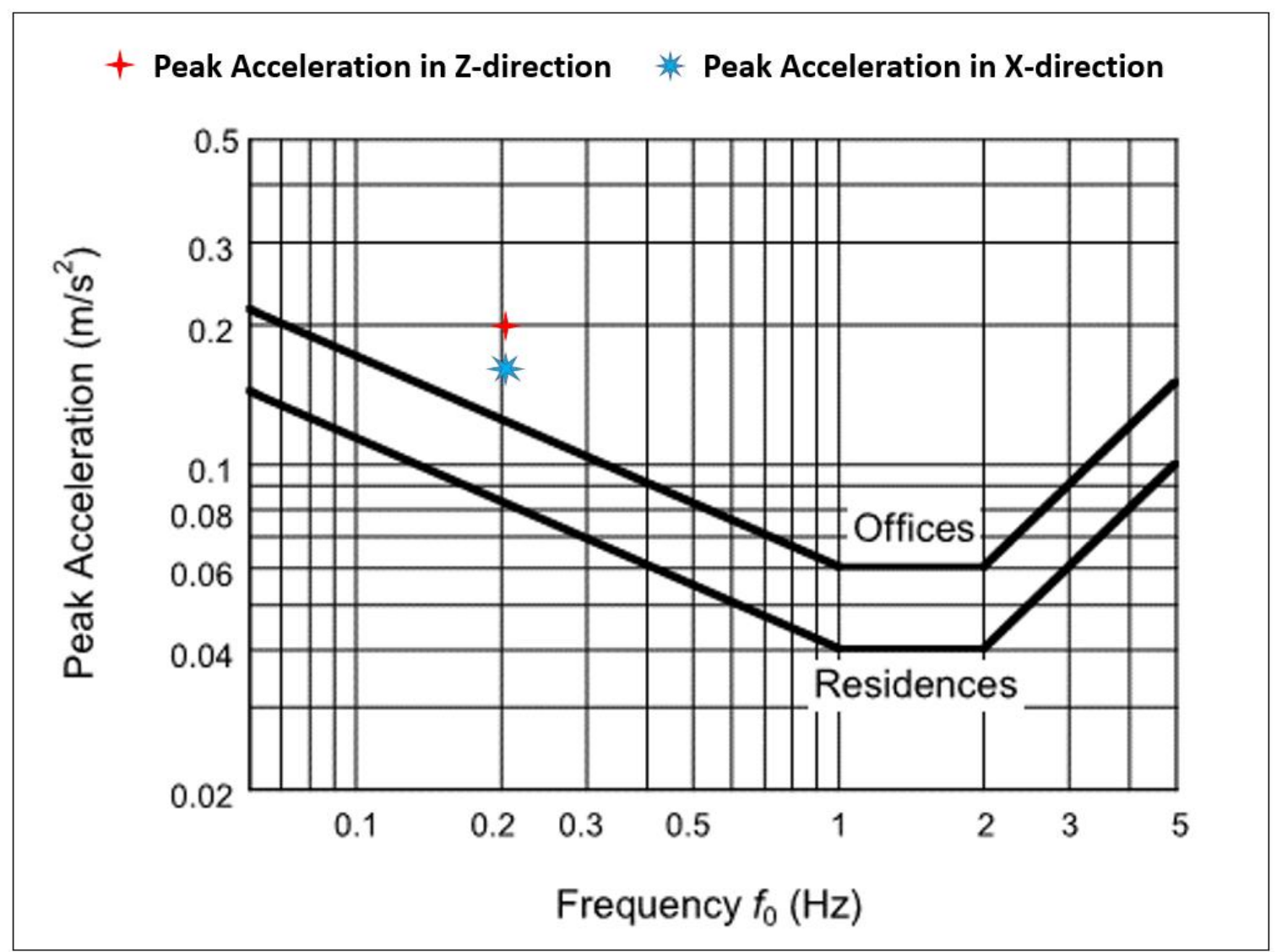

Figure 7-25. 10-year MRI peak acceleration responses of the 40-story building in $X$ and $Z$ directions in respect to basic evaluation curves for wind-induced vibration 


\section{CHAPTER VIII}

\section{SUMMARY AND CONCLUSIONS}

This chapter summarizes the conclusions of this dissertation. The finding of this research is presented in two sections: (1) Wind Testing: this section summarizes the conclusion of the wind pressure testing on a scaled high-rise building model; and (2) Wind Performance Assessments: this section summarizes the conclusions of dynamic simulation results and the performance-based evaluations of three steel moment frame high-rise buildings against the wind hazard.

\subsection{Wind Testing}

The objective of this experimental study was to investigate the characteristics of wind loading on a 47 -story building by conducting a set of $1 / 400$ scaled Wall of Wind tests. In these experiments, three models of (1) an isolated prismatic model, (2) an isolated full building model (including penthouse and building skirt) and (3) a full building model with surrounding buildings, were tested to study the effects of the building shape characteristics as well as the interference effects of surrounding buildings on wind loading. The effects on wind loading characteristics were investigated by providing wind pressure and force outputs such as; surface mean pressure coefficient distribution contours and mean and fluctuating base force measurements. Some conclusions from wind experiments are summarized as follows:

(1) The effect of the 47-story building's shape characteristics including penthouse and the skirt has been investigated through comparing the mean pressure coefficient distribution of the full building model (Test-\#2) with results from the prismatic model test (Test-\#1). The comparison indicated that minor building shape details may locally affect the local wind pressure of interest for cladding design. But they did not affect the overall wind loads very significantly.

(2) Mean and fluctuating base force coefficients, with reference to a fixed set of structural axes (X and Y) normal to building faces, were estimated for all three wind tests. The obtained results 
show that the 47-story building's shape details did not significantly affect the mean and fluctuating components of the wind force. Comparing the results obtained from the model surrounded by neighboring buildings (Test-\#3) with the other two tests indicated that the sheltering effect significantly decreased the mean and fluctuating component of the wind force for wind directions blocked by upstream buildings. Mean and fluctuating base force coefficient data from Test_\#3 suggest that the directions from $120^{\circ}$ to $180^{\circ}$ where the building gets the least sheltering from surrounding buildings constitute the critical wind directions.

(3) Comparison between the present wind testing, conducted in Wall of Wind facility at Florida International University, with wind tunnel data provided by TPU aerodynamic database for a model with the same aspect ratios showed an acceptable consistency of results from the two laboratories.

\subsection{Wind Performance Assessment}

The wind dynamic response of three steel moment frame high-rise buildings, including an existing 47-story building and two research cases, 30- and 40-story steel IMF buildings, were evaluated utilizing a performance-based assessment approach developed in this study. The motivation to study the 47-story building was due to the fact that the building survived Alicia Hurricane without significant structural damage, despite its primitive wind design in the early 1970s. Underestimated wind loads in addition to no consideration of drift criteria resulted in a relatively flexible building highly susceptible to wind dynamic excitations. Two 30- and 40-story steel IMF high-rise buildings designed according to current design approaches for both dominant seismic and wind loads were also evaluated. This study was also intended to evaluate the additional lateral resistance provided by allowing controlled nonlinearity in structural components along with consequent effects on serviceability and non-structural component performances. 
Analytical studies were performed by developing 3D nonlinear finite element models including elastic elements and concentrated plastic hinges, explicitly modeling the inelasticity in beams, columns, connections and panel zones. In addition to moment-resisting frames, gravity framing was modeled to incorporate its lateral resistance and stiffness into the global response of the building. Dynamic wind loads were acquired by carrying out wind pressure testing for the 47story building and using TPU wind tunnel database for the 30 - and 40 -story buildings. The measured dynamic wind loading was simulated by time histories of point loads at 256, 208 and 160 locations around the 47-, 40- and 30-story buildings respectively.

Nonlinear wind pushover analyses were conducted using the ASCE7 equivalent static wind loading pattern to evaluate the static nonlinear over-strength with respect to the design wind loads. The results indicate wind over-strength ratios equal to $2.5,3.5$ and 3.7 respectively for the 47-, 40- and 30-story buildings, in both principal directions of the buildings. over-strength The dominance of seismic loads in the design of the 30-story building may lead to relatively higher wind over-strength compared to the 40 -story building. Generally, the over-strength ratios indicate significant reserves in lateral resistance associated with allowing inelasticity in structural components.

The study on wind directionality indicated the critical dynamic response occurred in $120^{\circ}$ to $180^{\circ}$ wind directions for the 47-story building, in agreement with static estimation obtained directly from the wind test data. For the 30- and 40-story buildings, by using TPU wind tunnel data, a decreasing trend in all wind responses by changing wind angle from $0^{\circ}$ to $45^{\circ}$ is concluded.

Incremental dynamic analyses were carried out by conducting wind dynamic analyses for increasing wind hazard levels represented by the corresponding basic wind speed as defined in ASCE 7-10. For the 47-story building, the IDAs were repeated for six critical wind directions ranged from $120^{\circ}$ to $195^{\circ}$ with $15^{\circ}$ increments. For the 30 - and 40-story the IDAs were conducted 
for $0^{\circ}$ to $45^{\circ}$ wind directions in $15^{\circ}$ increments. Nonlinear response of plastic hinges in addition to global responses of story drift ratios and floor accelerations were evaluated for each wind hazard level in each wind direction.

Wind performance assessments of the three building were conducted by using the obtained IDA results in conjunction with a wind performance-based assessment approach proposed in this study. Four performance levels including three performance levels were considered: (1) Fully Operational (FO); (2) Limited Operations (LO); and (3) Life Safety (LS). This approach includes the evaluation of three types of building performance including: (1) Component Performance: indicating the level of force/deformation and corresponding damage in structural components; (2) Cladding performance under wind-induced shear deformation: indicating damage to the cladding system; and (3) Serviceability motion comfort performance: indicating the level of windinduced motion and its effects on occupant comfort including six performance levels; Not Perceptible; Threshold of Perceptibility; Target Level; Annoying; Very Annoying; and Intolerable. The corresponding performance criteria for all performance types and levels were also adopted from performance criteria recommended in the literature and codes. The estimated wind performances of the three building for different wind hazard levels in terms of the corresponding basic wind speed were as follows:

Component performance:

- 47-story building: (1) for less than $60 \mathrm{mph}$, FO performance level; (2) for 60 to $82 \mathrm{mph}$, LO performance level; (3) for 82 to $130 \mathrm{mph}$, LS performance level; and (4) for 130 to $136 \mathrm{mph}$, CP performance level.

- 40-story building: (1) for less than $70 \mathrm{mph}, \mathrm{FO}$ performance level; (2) for 70 to $125 \mathrm{mph}, \mathrm{LO}$ performance level; (3) for 125 to $170 \mathrm{mph}$, LS performance level. 
- 30-story building: (1) for less than $70 \mathrm{mph}$, FO performance level; (2) for 70 to $150 \mathrm{mph}, \mathrm{LO}$ performance level; (3) for 150 to $165 \mathrm{mph}$, LS performance level; and (4) for wind speeds greater than $165 \mathrm{mph}, \mathrm{CP}$ performance level.

Cladding performance to wind-induced shear deformation:

- 47-story building: (1) for less than $50 \mathrm{mph}$, FO performance level; (2) for 50 to $65 \mathrm{mph} \mathrm{LO}$ performance level; and (3) for 65 to $75 \mathrm{mph}$, LS performance level; and (4) for wind speeds greater than $75 \mathrm{mph}, \mathrm{CP}$ performance level.

- 40-story building: (1) for less than $72 \mathrm{mph}$, FO performance level; (2) for 72 to $93 \mathrm{mph}, \mathrm{LO}$ performance level; and (3) for 93 to $106 \mathrm{mph}$, LS performance level; and (4) for wind speeds greater than $106 \mathrm{mph}, \mathrm{CP}$ performance level.

- 30-story building: (1) for less than $51 \mathrm{mph}$, FO performance level; (2) for 51 to $80 \mathrm{mph}$, LO performance level; and (3) for 80 to $91 \mathrm{mph}$ LS performance level; and (4) for wind speeds greater than $91 \mathrm{mph}, \mathrm{CP}$ performance level.

Serviceability motion comfort performance:

- 47-story building: (1) no motion Perception for less than $24 \mathrm{mph}$ basic wind speed; (2) perceptibility threshold in 24 to $56 \mathrm{mph}$ basic wind speed range; and (3); Target Design: 60 to 64 mph basic wind speed; (4) annoying wind motion for 67 to 74 mph basic wind speed; (5) very annoying wind motion for 74 to $116 \mathrm{mph}$ wind speed; and (6) Intolerable wind motion for wind speeds greater than $116 \mathrm{mph}$ basic wind speed

- 40-story building: (1) no motion Perception for less than $15 \mathrm{mph}$ basic wind speed; (2) perceptibility threshold in 15 to $45 \mathrm{mph}$ basic wind speed range; and (3); Target Design: 62 to 76 mph basic wind speed; (4) annoying wind motion for 79 to 90 mph basic wind speed; (5) very annoying wind motion for 90 to $127 \mathrm{mph}$ wind speed; and (6) Intolerable wind motion for wind speeds greater than $127 \mathrm{mph}$ basic wind speed 
- 30-story building: (1) no motion Perception for less than $22 \mathrm{mph}$ basic wind speed; (2) perceptibility threshold in 22 to $68 \mathrm{mph}$ basic wind speed range; and (3); Target Design: 79 to 84 mph basic wind speed; (4) annoying wind motion for 87 to $96 \mathrm{mph}$ basic wind speed; (5) very annoying wind motion for 96 to $138 \mathrm{mph}$ wind speed; and (6) Intolerable wind motion for wind speeds greater than $138 \mathrm{mph}$ basic wind speed

For the 47-story building, the obtained performance assessment results were in a good agreement with building's records including the inspections performed after Hurricane Alicia. The conducted wind performance assessment of this under-designed high-rise building generally suggests that the remarkable nonlinear potential of the structural components, as well as neglected sources of lateral resistance such as gravity framing, enabled the building to sustain acceptable structural component performance in relatively high wind speeds. However, the intact flexibility of the building, in addition to softening associated with yielding of structural components, resulted in high shear deformation and floor accelerations, thus jeopardizing the serviceability and cladding performance of the building.

For the 30- and 40-story buildings the wind performance assessments showed high lateral resistance and acceptable structural component performance up to $160 \mathrm{mph}$ despite their $115 \mathrm{mph}$ design wind speed. Regarding the cladding performance under wind-induced shear deformation, both buildings failed to meet the suggested performance objectives. With respect to comfort serviceability, the 40-story building failed to meet the peak acceleration criteria for office buildings. However, the 30-story building almost met the criteria.

It is shown that the wind performance assessment is able to provide a full spectrum of serviceability status, despite the current serviceability check only based on 1-10 MRI winds. This is critical for the buildings designated to accommodate people during extreme winds to assure their acceptable serviceability performances. It is also shown that new buildings designed 
according to current wind design approach may fail to meet the cladding and serviceability performance objectives, however they may provide a very desirable structural performance during extreme winds.

It might be generally concluded that braced high-rise buildings, or moment frame high-rise buildings with added dampers and vibration mitigation systems, can more efficiently benefit from allowing controlled nonlinearity by simultaneously keeping other aspects of performance acceptable. It is also mainly concluded that using the performance-based engineering approach as implemented in this study assures efficient diagnostics of existing buildings as well efficient wind design of new buildings.

\subsection{Future Research}

This dissertation developed a wind performance assessment approach and implemented it to evaluate three steel moment frame high-rise buildings. The evaluations were carried out by conducting nonlinear dynamic response history analyses under wind loading to assess the structural response to different wind hazard levels. Extensive efforts were made to come up with a reliable analytical approach, perform massive finite element analyses and managing the huge amount of output data related to about one-hour of wind dynamic simulation. It was accomplished by adopting and implementing the knowledge and tools mainly developed in seismic engineering. Development of knowledge and analysis tools oriented for wind dynamic analyses will significantly improve PBWD and will promote it to practical implementation in wind evaluation and design.

To improve the accuracy of wind dynamic response simulation, the aerodynamic damping needs to be properly incorporated into the analytical model. To this end, the aerodynamic damping in two building's major directions can be evaluated by conducting wind tunnel testing on a scaled aeroelastic model in different wind directions. For each wind direction, the analytical model then 
needs to be calibrated to reflect the measured aerodynamic damping in two building's major directions.

While several wind performance-based engineering frameworks have been developed recently, they all may be considered relatively primitive. Comprehensive research is required to develop reliable performance objectives and acceptance criteria for wind response of high-rise buildings.

As already mentioned, damage to the cladding of high-rise building is the major concern regarding their performance during the hurricane and extreme wind events. Comprehensive research is needed to provide a performance-based assessment and a fragility evaluation framework for cladding systems.

In a further step, by advancing performance-based engineering in wind engineering and other hazard-related disciplines, a general multi-hazard performance-based engineering can be developed to simultaneously address the performance concerns associated with the different hazards. Future research to address the aforementioned knowledge gaps and promote a transition to practical implementation is recommended by the author. 


\section{REFERENCES}

AIJ (2004). AIJ Recommendations for Loads on Buildings, (AIJ-RLB-2004). Architectural Institute of Japan, Tokyo, Japan.

AISC. (2010a). Seismic provisions for structural steel buildings, (ANSI/AISC 341-10). American Institute of Steel Construction, Chicago, IL.

AISC. (2010b). Specification for structural steel buildings (ANSI/AISC 360-10). American Institute of Steel Construction, Chicago, IL.

ASCE. (2007). Seismic rehabilitation of existing buildings, (ASCE/SEI 41-06). American Society of Civil Engineers, Reston, VA.

ASCE. (2010). Minimum design loads for buildings and other structures, (ASCE 7-10). American Society of Civil Engineers, Reston, VA.

ASCE. (2014). Seismic rehabilitation of existing buildings (ASCE/SEI 41-13). American Society of Civil Engineers, Reston, VA.

Aswegan, K., Charney, F. A., \& Jarrett, J. (2015). Recommended procedures for damage-based serviceability design of steel buildings under wind loads. Engineering Journal-American Institute of Steel Construction, 52(1), 1-25.

ATC. (1996a). Seismic evaluation and retrofit of concrete buildings. Applied Technology Council, (ATC-40). Redwood City, CA.

ATC. (2009). Guidelines for seismic performance assessment of buildings, (ATC-58). Applied Technology Council, Redwood City, CA.

Azizinamini, A. (1985). Cyclic characteristics of bolted semi-rigid steel beam to column connections. Ph.D. Dissertation, University of South Carolina, Columbia, SC.

Bartoli, G., Ricciardelli, F., Saetta, A., \& Sepe, V. (2006). Performance of wind exposed structures. results of the PERBACCO project. Firenze University Press, Firenze, Italy.

Bernal, D. (1994). Viscous damping in inelastic structural response. Journal of Structural Engineering, 120(4), 1240-1254.

Borges, J. F. (1969). Observation of buildings subjected to dynamic loading. Laboratório Nacional de Engenharia Civil, Lisbon, Portugal.

Chang, F. (1973). Human response to motions in tall buildings. Journal of the Structural Division, 99(6), 1259-1272.

Charney, F. A. (2008). Unintended consequences of modeling damping in structures. Journal of Structural Engineering, 134(4), 581-592. 
Chenouda, M., \& Ayoub, A. (2008). Inelastic displacement ratios of degrading systems. Journal of Structural Engineering, 134(6), 1030-1045.

Chopra, A. K. (1995). Dynamics of structures. Prentice Hall, Englewood Cliffs, NJ.

Chopra, A. K., \& McKenna, F. (2016). Modeling viscous damping in nonlinear response history analysis of buildings for earthquake excitation. Earthquake Engineering \& Structural Dynamics, 45(2), 193-211.

Ciampoli, M., Petrini, F., \& Augusti, G. (2011). Performance-based wind engineering: Towards a general procedure. Structural Safety, 33(6), 367-378.

Coughey, T., \& Kelley, M. (1960). Classical normal modes in damped linear systems. Journal of Applied Mechanics, 269-271.

Dao, N. D., \& Ryan, K. L. (2013). Computational simulation of a full-scale, fixed-base, and isolated-base steel moment frame building tested at E-defense. Journal of Structural Engineering, 140(8), A4014005.

Deierlein, G., Reinhorn, A., \& Willford, M. (2010). Nonlinear structural analysis for seismic design, a guide for practicing engineers, (NIST GCR 10-917-5). National Institute of Standards and Technology. Gaithersburg, MD.

Elkady, A., \& Lignos, D. G. (2015). Effect of gravity framing on the overstrength and collapse capacity of steel frame buildings with perimeter special moment frames. Earthquake Engineering \& Structural Dynamics, 44(8), 1289-1307.

Elnashai, A., Elghazouli, A., \& Denesh-Ashtiani, F. (1998). Response of semi-rigid steel frames to cyclic and earthquake loads. Journal of Structural Engineering, 124(8), 857-867.

Emanuel, K. (2005). Increasing destructiveness of tropical cyclones over the past 30 years. Nature, 436(7051), 686-688.

Engelhardt, M., \& Husain, A. (1993). Cyclic-loading performance of welded flange-bolted web connections. Journal of Structural Engineering, 119(12), 3537-3550.

FEMA. (1997a). NEHRP guidelines for the seismic rehabilitation of buildings, (FEMA 273/274). Federal Emergency Management Agency, Washington, DC.

FEMA. (1997b). NEHRP guidelines for the seismic rehabilitation of buildings. (FEMA-273), Federal Emergency Management Agency, Washington, DC.

FEMA. (2000a). Pre-standard and commentary for the seismic rehabilitation of buildings, (FEMA-356). Federal Emergency Management Agency, Washington, DC.

FEMA. (2000b). State of the art report on connection performance, (FEMA 355D). Federal Emergency Management Agency, Washington, DC. 
FEMA. (2005). Improvement of nonlinear static seismic analysis procedures, (FEMA-440), Federal Emergency Management Agency, Washington, DC.

FEMA. (2009). Effects of strength and stiffness degradation on the seismic response of structural systems, (FEMA P-440A). Federal Emergency Management Agency, Washington, DC.

FEMA. (2012). Guidelines for design of structures for vertical evacuation from tsunamis, (FEMA P-646). Federal Emergency Management Agency, Washington, DC.

FEMA. (2013). Hurricane sandy in new jersey and new york, building performance observations, recommendations, and technical guidance, (FEMA P-942). Federal Emergency Management Agency, Washington, DC.

Flores, F. X., Charney, F. A., \& Lopez-Garcia, D. (2014). Influence of the gravity framing system on the collapse performance of special steel moment frames. Journal of Constructional Steel Research, 101, 351-362.

Gavin, H. P. (2014). Classical damping, non-classical damping, and complex modes. Class Notes for CEE, 5. Duke University, Durham, North Carolina.

Giuffrè, A., \& Pinto, P. (1970). Il comportamento del cemento armato per sollecitazioni cicliche di forte intensità. Giornale Del Genio Civile, 5(1), 391-408.

Goldenberg, S. B., Landsea, C. W., Mestas-Nunez, A. M., \& Gray, W. M. (2001). The recent increase in Atlantic hurricane activity: Causes and implications. Science, 293(5529), 474479.

Griffis, L. (2003). Serviceability limit states under wind load. Engineering Journal of American Institute of Steel Construction, 4(1), 1-16.

Griffis, L., Patel, V., Muthukumar, S., \& Baldava, S. (2012). A framework for performance-based wind engineering. Advances in Hurricane Engineering, 1205-1216.

Gupta, A., \& Krawinkler, H. (2000). Estimation of seismic drift demands for frame structures. Earthquake Engineering \& Structural Dynamics, 29(9), 1287-1305.

Gutierrez, C., Cresanti, R., \& Jeffrey, W. (2006). Performance of physical structures in hurricane Katrina and hurricane Rita: A reconnaissance report, (NIST Technical Note 1476). National Institute of Standards and Technology, Gaithersburg, MD.

Hall, J. F. (2006). Problems encountered from the use (or misuse) of Rayleigh damping. Earthquake Engineering \& Structural Dynamics, 35(5), 525-545.

Han, S. W., Moon, K., \& Ha, S. J. (2015). Seismic performance of high-rise intermediate steel moment frames according to rotation capacities of moment connections. International Journal of High-Rise Buildings, Vol 4(No 1), 45-55. 
Heintz, J., \& Mahoney, M. (2008). Guidelines for design of structures for vertical evacuation from tsunamis, (ATC-64). Applied Technology Council, (ATC-40). Redwood City, CA.

Ibarra, L., Medina, R., \& Krawinkler, H. (2002). Collapse assessment of deteriorating SDOF systems. Proceedings of the 12th European Conference on Earthquake Engineering, 9-13.

Ibarra, L. F., \& Krawinkler, H. (2005). Global collapse of frame structures under seismic excitations Pacific Earthquake Engineering Research Center. Berkeley. CA.

Ibarra, L. F., Medina, R. A., \& Krawinkler, H. (2005). Hysteretic models that incorporate strength and stiffness deterioration. Earthquake Engineering \& Structural Dynamics, 34(12), 14891511.

Irwin, H., Cooper, K., \& Girard, R. (1979). Correction of distortion effects caused by tubing systems in measurements of fluctuating pressures. Journal of Wind Engineering and Industrial Aerodynamics, 5(1-2), 93-107.

ISO, I. (2004). 10137: Bases for design of structures-serviceability of buildings and walkways against vibrations. International Standard Organization (ISO), Geneva, Switzerland.

Isyumov, N., \& Halvorson, R. (1984). Dynamic response of allied bank plaza during Alicia. Hurricane Alicia: One Year Later, 98-116.

Jin, J., \& El-Tawil. (2005). Seismic performance of steel panel zones. Journal of Structural Engineering, 131(No. 2), 250-258.

Kareem, A., \& Bashor, R. (2006). Performance of glass/cladding of high-rise buildings in hurricane Katrina. NatHaz Modeling Laboratory, University of Notre Dame, Notre Dame, IN.

Kim, K., \& Engelhardt M. (1995). Development of analytical models for earthquake analysis of steel moment frames. (No. Report No. PMFSEL 95-2). Department of Civil Engineering, University of Texas at Austin, Texas.

Kircher, C. A., Nassar, A. A., Kustu, O., \& Holmes, W. T. (1997). Development of building damage functions for earthquake loss estimation. Earthquake Spectra, 13(4), 663-682.

Kircher, C. A., Reitherman, R. K., Whitman, R. V., \& Arnold, C. (1997). Estimation of earthquake losses to buildings. Earthquake Spectra, 13(4), 703-720.

Krawinkler, H. (1978). Shear design of steel frame joints. Engineering Journal, 15(3)

Krawinkler, H., \& Venture, S. J. (2000). State of the art report on systems performance of steel moment frames subject to earthquake ground shaking. SAC Joint Venture, Federal Emergency Management Agency, Washington, DC.

Kunnath, S. K., Mander, J. B., \& Fang, L. (1997). Parameter identification for degrading and pinched hysteretic structural concrete systems. Engineering Structures, 19(3), 224-232. 
Kwon, D. K., Spence, S. M., \& Kareem, A. (2014). Performance evaluation of database-enabled design frameworks for the preliminary design of tall buildings. Journal of Structural Engineering, 141(10), 04014242.

Leon, R. T. (1990). Semi-rigid composite construction. Journal of Constructional Steel Research, 15(1-2), 99-120.

Leon, R. T., Hajjar, J. F., \& Shield, C. K. (1997). The effect of composite floor slabs on the behavior of steel moment-resisting frames in the Northridge earthquake. Composite Construction in Steel and Concrete III, 738-751.

Li, J., Ozbay, K., \& Bartin, B. (2014). Effects of hurricane Irene and sandy in new jersey: Evacuation traffic patterns. Transportation Research Board 93rd Annual Meeting, (14-3888)

Lignos, D. G., \& Krawinkler, H. (2010a). Deterioration modeling of steel components in support of collapse prediction of steel moment frames under earthquake loading. Journal of Structural Engineering, 137(11), 1291-1302.

Lignos, D. G., \& Krawinkler, H. (2010b). A steel database for component deterioration of tubular hollow square steel columns under varying axial load for collapse assessment of steel structures under earthquakes. Proc. 7th International Conference on Urban Earthquake Engineering (7CUEE), March, 3-5.

Lin, K., Tsai, K., Kong, S., \& Hsieh, S. (2000). Effects of panel zone deformations on cyclic performance of welded moment connections. XII WCEE, New Zealand, Doc, (1252)

Lindsay, B. R. (2010). Federal evacuation policy: Issues for congress, DIANE Publishing, Collingdale, PA.

Liu, J., \& Astaneh-Asl, A. (2000). Cyclic testing of simple connections including effects of slab. Journal of Structural Engineering, 126(1), 32-39.

Medina, R. A., \& Krawinkler, H. (2004). Seismic demands for no deteriorating frame structures and their dependence on ground motions, (Report No. 144), Stanford University. Stanford. CA.

Menegotto, M., \& Pinto, P. (1973). Method of analysis for cyclically loaded RC frames including changes in geometry and non-elastic behavior of elements under combined normal force and bending. IABSE Congress Reports of the Working Commission, 13.

Mishra, V. K. (2010). Impact of Hurricanes on Structures-a Performance Based Engineering View, Master Thesis, University of Central Florida, Orlando, FL.

Mooneghi, M. A., Irwin, P., \& Chowdhury, A. G. (2015). Exploratory studies on a bilinear aeroelastic model for tall buildings. Proceedings of the ICWE14, 14th International Conference on Wind Engineering, June 21-26, 
Mostaghel, N. (1999). Analytical description of pinching, degrading hysteretic systems. Journal of Engineering Mechanics, 125(2), 216-224.

Nader, M. N., \& Astaneh-Asl, A. (1996). Shaking table tests of rigid, semi-rigid, and flexible steel frames. Journal of Structural Engineering, 122(6), 589-596.

NEHRP. (2004). NEHRP recommended provisions for seismic regulations for new buildings and other structures (FEMA 450): Provisions Building Seismic Safety Council, National Institute of Building Sciences, Washington, DC.

Neuenhofer, A., \& Filippou, F. C. (1997). Evaluation of nonlinear frame finite-element models. Journal of Structural Engineering, 123(7), 958-966.

NIST. (2011). GCR 10-917-8 (2010) evaluation of the FEMA P-695 methodology for quantification of building seismic performance factors. National Institute of Standards and Technology, Gaithersburg, MD.

NOAA. (2011). NOAA technical memorandum NWS NHC-6: The deadliest, costliest, and most intense united states tropical cyclones from 1851 to 2010 (and other frequently requested hurricane facts). National Weather Service, National Hurricane Center, Miami, Florida,

PEER. (2015). Open system for earthquake engineering simulation (OpenSEES). Pacific Earthquake Engineering Research Center, Berkeley, CA.

PEER/ATC. (2010). Modeling and acceptance criteria for seismic design and analysis of tall buildings, (No. PEER/ATC 72-1 Report). Applied Technology Council, Redwood City, CA.

Petrini, F. (2009). A probabilistic approach to performance-based wind engineering (PBWE). Proc. Fifth European \& African conference on wind engineering (EACWE 5), Florence, Italy, July 19-23.

Popov, E. (1987). Panel zone flexibility in seismic moment joints. Journal of Constructional Steel Research, 8, 91-118.

Porter, K. A. (2003). An overview of PEER's performance-based earthquake engineering methodology. Proc. Ninth International Conference on Applications of Statistics and Probability in Civil Engineering, San Francisco, CA, July 6-9.

Ryan, K., Dao, N., Sato, E., Sasaki, T., \& Okazaki, T. (2012). NEES/E-defense base-isolation tests: Interaction of horizontal and vertical response. Proc. 15th World Conference on Earthquake Engineering, Lisbon, Portugal, September 24-48.

SEAOC. (2000). Performance Based Seismic Design Engineering, Committee 1995, Structural Engineers Association of California.

Sivaselvan, M. V., \& Reinhorn, A. M. (2000). Hysteretic models for deteriorating inelastic structures. Journal of Engineering Mechanics, 126(6), 633-640. 
Takemura, H., \& Kawashima, K. (1997). Effect of loading hysteresis on ductility capacity of reinforced concrete bridge piers. Journal of Structural Engineering, 43, 849-858.

Tsai, K., \& Popov, E. P. (1988). Steel beam-column joints in seismic moment resisting frames, (No. UCB/EERC-88/19). University of California, Berkeley, CA.

Webster, P. J., Holland, G. J., Curry, J. A., \& Chang, H. R. (2005). Changes in tropical cyclone number, duration, and intensity in a warming environment. Science, 309(5742), 1844-1846.

Wilson E. (2002). Three-dimensional static and dynamic analysis of structures; A physical approach with emphasis on earthquake engineering (Third Edition). Computers and Structures, Inc., Berkeley, CA.

Yeh, H. H., Robertson, I., \& Preuss, J. (2005). Development of design guidelines for structures that serve as tsunami vertical evacuation sites. Washington State Department of Natural Resources, Division of Geology and Earth Resources, Olympia, WA.

Zareian, F., Lignos, D., \& Krawinkler, H. (2010). Evaluation of seismic collapse performance of steel special moment resisting frames using FEMA P695 (ATC-63) methodology. Proceedings of Structures Congress ASCE, Orlando, FL, 12-14.

Zareian, F., \& Medina, R. A. (2010). A practical method for proper modeling of structural damping in inelastic plane structural systems. Computers \& Structures, 88(1), 45-53. 


\section{VITA}

\section{ALIREZA MOHAMMADI}

Born Tehran, Iran

2001-2005

B.Sc. Civil Engineering

Tabriz university

Tabriz, Iran

2006-2008

M.Sc. Civil Engineering/Earthquake Engineering

Sharif University of Technology

Tehran, Iran

2008-2012

Structural Engineer

Zelzeleh Mohaseb Consulting Engineers

Tehran, Iran

2012-2014

Graduate Assistant

Florida International University

Miami, Florida

2014-2016

Doctoral Candidate

Florida International

University Miami, Florida

\section{PUBLICATIONS AND PRESENTATIONS}

Khonsari, S.V., England, G.L., Mohammadi, A. (2009). Behavior of an Innovative Universal Structural Connection under Monotonic and Cyclic Shear Loading. In Proc. OMAE 2009, Paper No. 80047, May 31-June 5, Honolulu, Hawaii.

Mohammadi, A., Azizinamini, A. (2013). Evaluation of the effect of elimination of crosses frames between construction phases in Phase and Widening Construction of Steel Bridges. Poster presented at Graduate Research Day, February 22, 2013.

Mohammadi, A. Azizinamini, A., (2014). "Evaluating Implementation of Alternative Cross Frame Configurations between Construction Phases in Phased and Widening Construction of Steel I-Girder Bridges", Poster presented at FIU Engineering Expo, February 21, 2014.

Mohammadi, A., Yakel A., Azizinamini, A. (2014). Phase and Widening Construction of Steel Bridges. Florida Department of Transportation, 2014. Web. 25 July 2014. http://www.dot.state.fl.us/research-center/Completed_Proj/Summary_STR/FDOT-BDK80-97728-rpt.pdf

Mohammadi, A., Gull, J. H., Taghinezhad, R., Azizinamini, A. (2014). Assessment and Evaluation of Timber Piles Used in Nebraska for Retrofit and Rating. Nebraska Department of Roads, 2014. Web. 11 Aug. 2014.

http://www.transportation.nebraska.gov/mat-n-tests/research/BridgeOther/FinalReportM306.pdf 
Mohammadi, A., Yakel, A., Gull, J. H., \& Azizinamini, A. (2015). Alternative Framing Strategies for Steel I-Girder Phase 1 Construction Projects to Alleviate Fit-Up Issues. In Transportation Research Board 94th Annual Meeting (No. 15-4039).

Gull, J. H., Mohammadi, A., Taghinezhad, R., \& Azizinamini, A. (2015). Experimental Evaluation of Repair Options for Timber Piles. Transportation Research Record: Journal of the Transportation Research Board, (2481), 124-131.

Mohammadi, A. Azizinamini, A. (2015). Utilizing High-rise Buildings as Emergency Shelters During Extreme Wind Events. FIU CEE Research Day, February 27, 2015.

Mohammadi, A., Taghinezhad, R., Garber, D., \& Azizinamini, A. (2015). A Review of three Ongoing Researches in ABC-UTC Center at Florida International University. In UTC Conference for the Southeastern Region. Birmingham, Alabama, March 26-27, 2015.

Pham, H., Gull, J., Mohammadi, A., and Azizinamini A., (2016) Managing Florida's Fracture Critical Bridges - Phases I \& II. Florida Department of Transportation, 2016. Web. May 2016. http://www.dot.state.fl.us/researchcenter/Completed Proj/Summary MNT/FDOT-BDV29-97717-rpt.pdf. 